\title{
Structural and Magnetic Properties of the Glass-Forming Alloy $\mathrm{Nd}_{60} \mathrm{Fe}_{30} \mathrm{Al}_{10}$
}

\author{
Dissertation \\ zur Erlangung des Doktorgrades \\ der Mathematisch-Naturwissenschaftlichen Fakultäten \\ der Georg-August-Universität zu Göttingen
}

vorgelegt von

\section{Alberto Bracchi}

aus Montegiorgio (Italien)

Göttingen 2004 
Referent

Korreferent

Tag der mündlichen Prüfung:
PD Dr. Susanne Schneider

Prof. Dr. Konrad Samwer

18. November 2004 

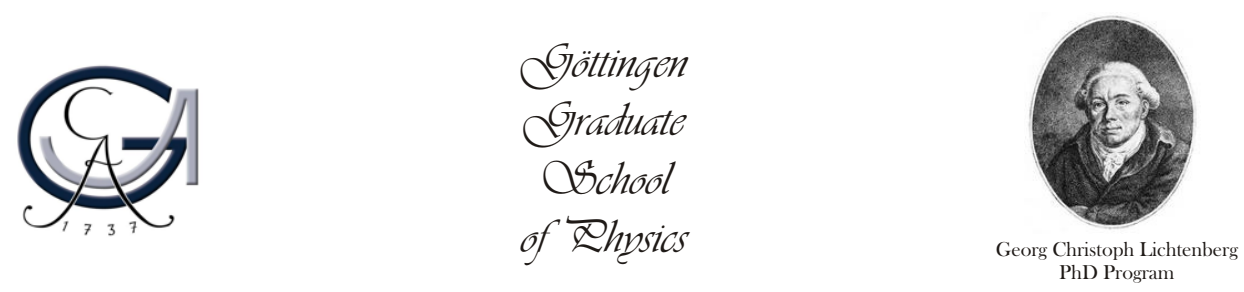

\title{
Structural and Magnetic Properties of the Glass-Forming Alloy $\mathrm{Nd}_{60} \mathrm{Fe}_{30} \mathrm{Al}_{10}$
}

\section{PhD Thesis}

\author{
of the Philosophical Doctoral degree \\ of the Georg-August-Universität of Göttingen: \\ Mathematical and Natural Sciences Faculties \\ and Graduate School of Physics
}

by

\section{Alberto Bracchi}

from Montegiorgio (Italy)

Göttingen (2004) 

When a book and a head collide and a hollow sound is heard, must it always have come from the book?

(Georg Christoph Lichtenberg, 1773-1775)

Pe' cantà, ce vo' la voce!

(Ieppa, 1992) 



\section{Kurzfassung}

\section{Mikrostrukturelle und magnetische Eigenschaften der glasbildenden Legierung $\mathrm{Nd}_{60} \mathrm{Fe}_{30} \mathrm{Al}_{10}$}

Strukturelle und thermophysikalische Untersuchungen an mehrkomponentigen, massiven metallischen Gläsern werden seit deren Entdeckung im Jahr 1990 durchgeführt, dabei wurde zunächst besonderer Wert auf die chemische und strukturelle Homogenität der Proben gelegt [22]. Viele Experimente haben aber gezeigt, dass diese mehrkomponentigen eutektischen glasbildenden Legierungen zur Entmischung in der (unterkühlten) Schmelze neigen. Erste Arbeiten von der Gruppe um A. Inoue 80 zeigen, dass massive glasbildende $\mathrm{Nd}_{60} \mathrm{Fe}_{30} \mathrm{Al}_{10}$ Legierungen hartmagnetisches Verhalten aufweisen. Wang et al. konnten zeigen, dass selbst aus der Schmelze schnell abgekühlte $\mathrm{Nd}_{60} \mathrm{Fe}_{30} \mathrm{Al}_{10}$ Gläser eher hartmagnetische Eigenschaften mit einer Koerzitivfeldstärke $H_{c}$ von $59 \mathrm{kA} / \mathrm{m}$ bei Raumtemperatur besitzen 168.

In der vorliegenden Arbeit werden die strukturellen und magnetischen Eigenschaften der glasbildenden Legierung $\mathrm{Nd}_{60} \mathrm{Fe}_{30} \mathrm{Al}_{10}$ systematisch untersucht. Die Tendenz dieser Legierung zur Phasenseparation und zur Bildung eines intrinsischen Komposits ermöglicht es, die strukturellen Charakteristika durch Änderung der bei der Herstellung verwendeten Abkühlrate zu variieren. Mittels verschiedener Präparationsmethoden sind Proben mit Abkühlraten von etwa $10 \mathrm{~K} / \mathrm{s}$ bis zu $10^{10} \mathrm{~K} / \mathrm{s}$ hergestellt worden. Durch hochenergetische Röntgenbeugung, Kleinwinkelneutronenstreuung und hochauflösende TransmissionsElektronen-Mikroskopie wurden die Struktur und die Morphologie der verschiedenen Proben untersucht, während die magnetischen Eigenschaften durch SQUID- und VSMMagnetometrie, Mössbauer Spektroskopie und polarisierte Kleinwinkelneutronenstreuung charakterisiert wurden.

Die Ergebnisse zeigen, dass die magnetischen Eigenschaften der $\mathrm{Nd}_{60} \mathrm{Fe}_{30} \mathrm{Al}_{10}$ Komposite stark von den strukturellen Eigenschaften abhängen. Ihr Zusammenspiel lässt sich gut mit Pinning Effekten der Domänenwände interpretiert werden. Die Temperaturabhängigkeit der Koerzitivfeldstärke kann sehr gut durch das Pinning Modell von Gaunt 63 aus dem Jahr 1983 beschrieben werden. Somit lässt sich das Pinning der ferromagneti- 
schen Phase an der Grenzfläche der in-situ gebildeten paramagnetischen Nanokristalle experimentell nachweisen. 


\section{Contents}

Title i

German Abstract vii

Table of Contents

1 Introduction 1

2 Magnetic Metallic Glasses and Intrinsic Composites 5

2.1 Amorphization of metals and metallic alloys . . . . . . . . . . 5

2.1.1 Historical Background . . . . . . . . . . . . 5

2.1 .2 Glassy Materials ............... 6 6

2.1.3 Phenomenological Conditions for Glass Formation . . . . . . . . 8

2.2 Preparation Methods of Metallic Glasses . . . . . . . . . . . . . 12

2.2.1 Melt Quenching . . . . . . . . . . . . . 12

2.2.1.1 "Silver Boat" Induction Melter . . . . . . . . . . . 13

2.2.1.2 Arc Melting . . . . . . . . . . . . . 14

2.2.1.3 Copper Mold Casting . . . . . . . . . . . . 15

2.2.1.4 Splat Quenching ............... 16

2.2.2 Vapor Deposition . . . . . . . . . . . . . . 17

2.2.2.1 Electron-Beam Evaporation . . . . . . . . . . 18

2.3 Intrinsic Composites . . . . . . . . . . . . . . . . . . 19

2.3.1 Physical properties of Intrinsic Composites . . . . . . . . . . . 20

2.3.1.1 Mechanical properties ............ 20. 20

2.3.1.2 Magnetic properties .............. 21

3 Thermal Stability of Intrinsic Composite Materials 23

3.1 Nucleation-and-Growth . . . . . . . . . . . . . 24

3.1.1 Reaction Kinetics in Differential Scanning Calorimetry . . . . . . 28

3.1.2 Continuous-Heating-Transformation (CHT) Diagram . . . . . . . 32

3.2 Coarsening . . . . . . . . . . . . . . . . . . . 33

3.2.1 Experimental Investigation of Coarsening in Composite Materials . 36 
4 Magnetism in Amorphous and Composite Materials 39

4.1 Effect of Disorder on Magnetic Properties . . . . . . . . . . . . . . . 39

4.1.1 Effects of Disorder on Magnetic Moments . . . . . . . . . . . . 40

4.1.2 Effects of Disorder on Exchange Interactions . . . . . . . . . . 41

4.1.3 Effects of Disorder on Magnetic Anisotropy . . . . . . . . . . . 43

4.2 Collective Magnetic Order in Disordered Materials . . . . . . . . . . . . 45

4.3 Random Axial Anisotropy Model (RAM) . . . . . . . . . . . . 48

4.3 .1 Magnetic Domains . . . . . . . . . . . . . 52 52

4.4 Magnetization Mechanisms . . . . . . . . . . . . . 54

4.5 Coercivity Mechanisms . . . . . . . . . . . . . . 555

4.5.1 Domain Wall Pinning. . . . . . . . . . . 56 56

4.5.1.1 Gaunt's Model .............. 557

4.5.1.2 Origin of Pinning Effetcs . . . . . . . . . 62

5 Experimental Methods and Techniques 63

5.1 SQUID Magnetometer . . . . . . . . . . . . . . . 64 64

5.1 .1 AC Susceptibility . . . . . . . . . . . . . 69 69

5.2 Vibrating Sample Magnetometer (VSM) . . . . . . . . . . . . . 70

5.3 Small Angle Neutron Scattering . . . . . . . . . . . . . . . 74

5.3 .1 Neutron Radiation ................... 74

5.3 .2 Nuclear Scattering . . . . . . . . . . . . 76

5.3 .3 Magnetic Scattering . . . . . . . . . . . . . . 83

5.3.4 Separation of nuclear and magnetic scattering . . . . . . . . 85

5.3.5 Polarized Small Angle Neutron Scattering (pSANS) . . . . . . . 87

5.3 .6 Neutron Sources . . . . . . . . . . . . . . . 90 90

5.4 High Resolution Transmission Electron Microscopy (HRTEM) . . . . . . 92

5.4 Sample Preparation . . . . . . . . . . . . . 94

5.5 Differential Scanning Calorimetry (DSC) . . . . . . . . . . . . . 94

5.6 High Energy Wide Angle X-Ray Scattering (HE-WAXS) . . . . . . . . . 96

5.6.1 Directions and Intensities of diffracted X-Ray Beam . . . . . . . . . 99

5.7 Annealing Methods . . . . . . . . . . . . . . 103

6 Results and Discussion 105

6.1 Bulk Samples . . . . . . . . . . . . . . . . . . 105

6.1.1 X-Ray Diffraction and Transmission Electron Microscopy . . . . . . 106

6.1.2 Small-Angle Neutron Scattering . . . . . . . . . . . . 111

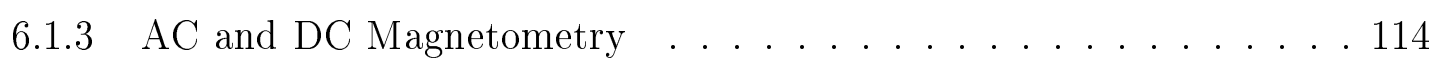

6.1.4 Coercivity Mechanisms . . . . . . . . . . . . . 121

6.1.5 Curie Temperature and Critical Behavior . . . . . . . . . . . 126

6.1.6 Mössbauer Spectroscopy . . . . . . . . . . . . . . . . . . 130 
6.1.7 Differential Scanning Calorimetry and Thermal Stability . . . . . 131

6.1 .8 Summary . . . . . . . . . . . . . . . . 136

6.2 Master Alloy . . . . . . . . . . . . . . . . . . . . . . 139

6.2.1 X-Ray Diffraction . . . . . . . . . . . . . 139

6.2.2 Differential Scanning Calorimetry and Thermal Stability . . . . . 140

6.2 .3 DC Magnetometry . . . . . . . . . . . . . . . . . . 142

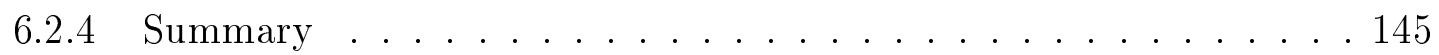

6.3 Splat-cooled Foil . . . . . . . . . . . . . . . . . . . . 146

6.3.1 X-Ray Diffraction and Transmission Electron Microscopy . . . . . . 146

6.3.2 Differential Scanning Calorimetry and Thermal Stability . . . . . 149

6.3.3 AC and DC Magnetometry . . . . . . . . . . . . . 151

6.3.4 Small-Angle Neutron Scattering . . . . . . . . . . . . . . 155

6.3.5 Mössbauer Spectroscopy . . . . . . . . . . . . . . . 157

6.3.6 Coercivity Mechanisms . . . . . . . . . . . . . 158

6.3 .7 Summary ........................... 163

6.4 Thin Film . . . . . . . . . . . . . . . . . . 164

6.4.1 X-Ray Diffraction and Transmission Electron Microscopy . . . . . . 164

6.4 .2 DC Magnetometry . . . . . . . . . . . . . . . 167

6.4.3 Coercivity Mechanisms . . . . . . . . . . . . 169

6.4 .4 Summary . . . . . . . . . . . . . . . . 170

6.5 Annealed Samples . . . . . . . . . . . . . . . . 171

6.5.1 Annealing at 500 and $630 \mathrm{~K} \ldots \ldots . \ldots . \ldots 172$

6.5 .2 Annealing at $800 \mathrm{~K} \ldots \ldots \ldots \ldots \ldots$

7 Concluding Remarks

7.1 Summary and Conclusions . . . . . . . . . . . . . . . 177

7.2 Outlook ............................ 180

A Fitting Processes 183

B SANS Form Factors

C Mass Fractals 187

Bibliography

Publications $\quad 203$

Acknowledgments 205

\begin{tabular}{ll} 
Curriculum Vitae & 209 \\
\hline
\end{tabular} 



\section{Chapter 1}

\section{Introduction}

Magnetic materials have a long history of application and have nowadays an invaluable importance in a wide variety of devices and equipments (e.g., power transformers, data storage, etc.). Their properties range from hard magnetic to soft magnetic, insulating to metallic, but magnetic materials have always been exclusively crystalline. However, one of the newest addition to the class of magnetic materials is not crystalline, but amorphous [53,56. The amorphous metallic alloys, sometimes called metallic glasses or glassy metals, are primary obtained by rapid cooling from the melt or from the vapor in such a way that the "disordered" structure of the liquid is preserved in a frozen state. The emergence of this new class of materials opened the way to new technological applications of magnetic alloys and represented also a significant challenge to the scientific understanding of magnetic materials since many properties could not be understood in the framework of the existing theories which mostly presume crystalline symmetry and lattice periodicity [53. The ferromagnetic amorphous alloys containing a large concentration of Fe or Co generally show excellent soft magnetic properties, equivalent or even superior to the conventional crystalline soft magnetic materials. Similarly, the hard magnetic properties have also attracted the scientific and technological interest and many efforts have been spent to understand the hard magnetic behavior of crystalline permanent magnets as, for example, the $\mathrm{Sm}-\mathrm{Co}$ and $\mathrm{Nd}-\mathrm{Fe}-\mathrm{B}$ alloys. In the last decades the interest in magnetic rare earth-transition metals $(\mathrm{RE}-\mathrm{TM})$ materials resulted then in intense activities on the study of their amorphous counterparts: the RE-TM amorphous alloys and, in particular, the binary Nd-Fe system. However, due to the high critical cooling rate showed by those alloys, only thin films or ribbons could be prepared and successfully studied.

In the last few years the interest on metallic glasses has further grown due to the possibility to produce multicomponent alloys in bulk form and to the outstanding mechanical properties which they showed. They were however limited to nonmagnetic systems and, only recently, magnetic bulk glasses have been developed by Inoue [78, 80, 81 and by Shen and Schwarz [143]. Among these, the $\mathrm{Nd}-\mathrm{Fe}-\mathrm{Al}$ system is probably the best known for the excellent hard magnetic properties. Structural investigations have shown however that 
the samples are not fully amorphous but intrinsic composites constituted of dispersed nanocrystals embedded in a remnant amorphous matrix [139. In this context, Hays 73 recently reported that several mechanical features of bulk glasses can be improved dramatically by reinforcing the amorphous matrix with a ductile dendritic crystalline phase.

Following the path used for the design of glasses with unique mechanical properties, this work intends to show that the magnetic properties can also be designed by varying the microstructure of the intrinsic composites and, hence, by tailoring morphology and length scales of the in situ formed nanocrystals. Two are the approaches usually proposed to control the microstructure of an intrinsic composite: to vary the chemical composition of the alloy (or to dope it with additional elements), and, for a fixed composition, to change the cooling rate at which the specimens are quenched. For this scope, different preparation methods and techniques are usually necessary. This thesis will prove that it is possible to prepare magnetic intrinsic composites from the glass-forming composition $\mathrm{Nd}_{60} \mathrm{Fe}_{30} \mathrm{Al}_{10}$ and to successfully tailor the microstructure of the specimens by changing the cooling rates during the solidification process and by post-preparation thermal treatments. For this scope bulk samples, splat-cooled foils, and thin films are produced by the use of four different methods ranging from the melt quenching to the vapor deposition. Structural, thermodynamical and magnetic properties of as-prepared and annealed samples are investigated by employing different characterization techniques as transmission electron microscopy with EDX analysis, polarized and unpolarized small-angle neutron scattering, SQUID and VSM magnetometry, high-energy x-ray scattering, and differential scanning calorimetry.

\section{Why the Nd-Fe-Al System?}

Two important prerequisites makes the $\mathrm{Nd}_{60} \mathrm{Fe}_{30} \mathrm{Al}_{10}$ alloy a good candidate for the study of the interplay between structural characteristics and hard magnetic properties: it belongs to the family of the RE-TM systems and it has shown a higher glass-forming ability compared to the binary $\mathrm{Nd}-\mathrm{Fe}[80]$.

From the technological point of view, the product $\left(4 \mu_{0} M \cdot H\right)_{\max }$ is usually considered the most important parameter of a ferromagnetic material since it represents the energy dissipated during a hysteresis loop and it expresses the tendency of the system to be easily magnetized or demagnetized. $\left(4 \mu_{0} M \cdot H\right)_{\max }$ is sometimes approximated by the product $\left(4 \mu_{0} M_{\max } \cdot H_{c}\right)$, where $M_{\max }$ is the magnetization for which $\left(4 \mu_{0} M \cdot H\right)$ is maximal, and $H_{c}$ is the coercive field. If $\left(4 \mu_{0} M \cdot H\right)_{\max }$ is small (low $\left.H_{c}\right)$, the material can be magnetized with a low external field and it is said to be magnetically soft, while if $\left(4 \mu_{0} M \cdot H\right)_{\max }$ is large (high $H_{c}$ ), the material behaves like a permanent magnet and it is said to be magnetically hard. Therefore, the search of a good hard magnetic material induces the efforts towards the family of the RE-TM alloys due to the high magnetic moment (high $M_{\max }$ ) and 
large magnetocrystalline anisotropy (high $H_{c}$ ) of the RE atoms and to the possibility of tailoring their intrinsic magnetic properties by alloying them with TM elements.

On the other hand, what makes a ferromagnetic material composed of crystalline precipitates in an amorphous matrix (intrinsic composite) even more attractive than a crystalline alloy or a sintered magnet is the possibility to tailor "on demand" its magnetic properties by employing different preparation methods (i.e., cooling rates) or opportune post-preparation thermal treatments. In this context, the higher the glass-forming ability of an alloy, the larger the temperature interval in which the structural evolution can be controlled without the samples fully crystallize.

Hence, the choice of the $\mathrm{Nd}_{60} \mathrm{Fe}_{30} \mathrm{Al}_{10}$ system is motivated by the technological interest for a material with large $\left(4 \mu_{0} M \cdot H\right)_{\max }$ and justified by the need for an alloy whose intrinsic properties can be successfully tailored by a controlled evolution of its microstructure. For this scope, $\mathrm{Nd}_{60} \mathrm{Fe}_{30} \mathrm{Al}_{10}$ bulk samples, foils and thin films were produced employing different preparation methods which allow cooling rates ranging from some $\mathrm{K} / \mathrm{s}$ up to $10^{10} \mathrm{~K} / \mathrm{s}$. In such way the specimens microstructure can be strongly influenced and cooling rate-dependent magnetic properties are expected.

\section{Aims, Scope, and Organization}

This thesis will show that the magnetic properties of $\mathrm{Nd}_{60} \mathrm{Fe}_{30} \mathrm{Al}_{10}$ composites strongly depend on the structural properties and, hence, that by tailoring the latter it is possible to control the first. The study focuses mainly on the magnetic characterization of the asprepared samples and on the mechanisms which control their magnetic properties: magnetic pinning effects and thermal activation processes of the domain walls motion. Their origin and the interplay with the heterogeneous microstructure, typical of intrinsic composite materials, are then explained on the basis of the experimental results. Afterwards, the issues of the samples thermal stability and of the evolution of their microstructure upon annealing are considered, and the resultant variation of the magnetic properties are used to verify the conclusions previously formulated for the as-prepared specimens and to evaluate the possible use of the $\mathrm{Nd}_{60} \mathrm{Fe}_{30} \mathrm{Al}_{10}$ alloy in technological applications.

The thesis is therefore structured as follows. The concept of glass-forming ability of a metallic alloy and the definitions of glass and intrinsic composite material are presented in chapter 2. In the same section the different methods used for the preparation of the $\mathrm{Nd}_{60} \mathrm{Fe}_{30} \mathrm{Al}_{10}$ specimens are described highlighting, in particular, those device characteristics which may influence the solidification paths and, ultimately, the cooling rates.

Chapter 3 presents several theoretical models facing the problem of the thermal stability of metastable systems, of which metallic glasses and intrinsic composites are an important example. The attention will be primarily focused on the crystallization process and on the crystal growth, which play a fundamental role on the microstructure evolution 
of the alloy under examination in this work.

The magnetic properties of an amorphous magnet are discussed in chapter 4 emphasizing the interplay between magnetic behavior and compositional/microstructural disorder. Such interplay is often responsible for collective magnetic order characterized by non-collinear moment and by random axial anisotropy and it has been proved to be particularly effective in the case of RE-TM alloys. For this reason, the random anisotropy model of Harris, Pliske and Zuckermann $|72|$ is briefly outlined and the relative extension of the concept of magnetic domain is introduced. Finally, pinning processes of the domain walls are then discussed as possible coercivity mechanism in non-homogeneous systems and, in this context, the domain-walls pinning model of Gaunt 63 is described in details.

Chapter 5 deals with the presentation of the measurement devices and the analysis employed for the characterization of the $\mathrm{Nd}_{60} \mathrm{Fe}_{30} \mathrm{Al}_{10}$ specimens. A detailed description is dedicated in particular to the SQUID magnetometer, the small angle neutron scattering, and the high-energy $\mathrm{x}$-ray diffraction which played the major role among the different techniques used during this work.

The experimental results concerning both magnetic and structural characterizations of all the $\mathrm{Nd}_{60} \mathrm{Fe}_{30} \mathrm{Al}_{10}$ samples are presented and discussed in chapter 6 . The different as-prepared specimens (i.e., characterized by different preparation methods and, hence, cooling rates) are separately introduced in the first sections while the last paragraph includes the investigation on the annealed samples and, hence, on the evolution of the physical properties upon thermal treatment.

The conclusions reached during this thesis are then summarized in chapter 7 and an outlook about further possible investigations on the $\mathrm{Nd}_{60} \mathrm{Fe}_{30} \mathrm{Al}_{10}$ system is presented in the last section of the chapter. 


\section{Chapter 2}

\section{Magnetic Metallic Glasses and Intrinsic Composites}

Metallic glasses are a relatively new class of materials which have however attracted the interest of many research groups. Many efforts have been spent during the last few decades trying to understand their properties and to employ them in technological applications. Many outstanding results have been already obtained but the physical explanation of the amorphization process of metals and metallic alloys is still a matter of dispute. Chapter 2 will try to introduce to this problematic starting from a historical background and concluding with a description of those theories which try to give a physical interpretation of glass formation (see section 2.1. Section 2.2 is then dedicated to the preparation methods of metallic glasses and, in particular, will describe the techniques used during this work for the production of the $\mathrm{Nd}_{60} \mathrm{Fe}_{30} \mathrm{Al}_{10}$ specimens. Finally, section 2.3 presents the class of the intrinsic composite materials which has been recently defined for glass-forming metallic alloys prepared under non-ideal conditions. The physical and technological relevance of composites is highlighted with some examples of their outstanding properties.

\subsection{Amorphization of metals and metallic alloys}

Amorphization of metals or metallic alloys is a topic that we cannot completely face without asking two general questions: What is a glass? and Which are the mechanism controlling the glass formation? The answers are still argument of controversial discussion in the scientific community, although the amorphous state of matter has been now studied for several decades. We will try to deal with this problematic in the following sections [66.

\subsubsection{Historical Background}

The study of the vitreous state of matter is a very old branch of physics, and human being has investigated and used (insulating) glasses (i.e., $\mathrm{SiO}_{2}$ ) for ages. On the contrary, 
metallic glasses are relatively new due to the fact that thermal evaporation and electron deposition have been known to yield amorphous films with any sharp x-ray diffraction only since the beginning of the 20th century [96. Moreover the first glassy films were more an accidental product and remained poorly characterized for a long time until a more systematic study on thin films prepared by vapor deposition at cryogenic temperatures was carried out by Hilsch and collaborators in the year 1954 21, 76.

The first ferromagnetic amorphous film $(\mathrm{Co}-\mathrm{Au})$ was obtained in 1965 by Mader and Nowick 111 who demonstrated, in this way, that ferromagnetism can exist even without lattice periodicity, as it was predicted theoretically in 1960 by Gubanov [67]. About 10 years later Fujime [53] and Felsch [56,57] succeeded in producing nominally pure amorphous thin films of $\mathrm{Fe}, \mathrm{Cr}$ and other transition metals by vapor deposition at $4.2 \mathrm{~K}$. The films were however metastable, stayed amorphous only at cryogenic temperatures, and crystallized when they were brought to room temperature. An amorphous alloy of a controlled composition which was stable even at room temperature was obtained quite accidentally by Duwez and his group in 1960 by rapid quenching of a molten metallic alloy while they were looking for new metastable crystalline phases in the Au-Si binary system [52,93. This opened the way to the production of bulk amorphous samples and a strong growth of the field followed rather quickly [86]. The first bulk amorphous magnetic material was found by Duwez six years later by replacing a non-magnetic element of a glass-forming alloy with a magnetic one and demonstrating that the Pd-Fe-Si alloy shows ferromagnetism [159]. The first amorphous soft magnetic material (with a coercivity of only 3 mOe) was found in 1975 by Egami [54] and a second rapid expansion in research activities followed under the impulse of possible industrial and commercial applications. The first material showing hard magnetic behavior and an amorphous-like structure was announced in 1996 by Inoue [80. The investigated composition was $\mathrm{Nd}_{60} \mathrm{Fe}_{30} \mathrm{Al}_{10}$. Since the first publication, many efforts have been spent trying to characterize structure and magnetic properties of this glass-forming alloy and this work represents one of them.

\subsubsection{Glassy Materials}

What is a glass? A Glass is often defined as a solid with disordered or aperiodic atomic structure (amorphous structure). However this definition is not satisfactory since even a crystalline solid with a high density of lattice defects or materials composed of nanosized crystals may satisfy it and even $\mathrm{x}$-ray or electron diffraction patterns might show broad diffraction haloes (instead of sharp Debye lines) leaving unanswered the question of whether this pattern originates from the glassiness of the structure or from the grain sizes being so small.

Historically, two approaches have tried to give a physical definition of glass: the first is a strictly phenomenological approach based upon carefully chosen experimental observa- 
tions, while the second is a theoretical approach involving the construction of an atomistic model of glass.

Phenomenologically a glass is a frozen liquid [160|. Certain composition ranges of metallic alloys when rapidly cooled do not crystallize at the melting temperature $T_{m}$ and can be further cooled down (undercooled) such that no phase transition is observed. The viscosity $\eta$ of an undercooled liquid is a strong function of temperature increasing rapidly but continuously from $T_{m}$ by more than ten orders of magnitude towards the glass transition temperature $\left(T_{g}\right)$ which is empirically defined as the temperature at which $\eta$ reaches $10^{13}$ Poise and the system behaves like a solid (kinetic arrest) 53. The glass transition is a spatially homogeneous process which proceeds without nucleation and differs therefore from the crystallization of a liquid phase which is a discontinuous and inhomogeneous first-order transition. In fact, when a liquid crystallizes near $T_{m}$ the viscosity changes discontinuously by several orders of magnitude, the interface between the liquid and the crystalline solid is well defined and the solidification proceeds by the motion of this interface. For a glass those processes are hampered and a glass appears therefore as frozen liquid, or an amorphous solid which exhibits a glass transition and, although both the glass transition and crystallization are actually the solidification of a liquid, they can be clearly distinguished by appropriate measurements of volume, specific heat, viscosity, expansion coefficient, etc. (see figure 2.1). Experimentally the glass
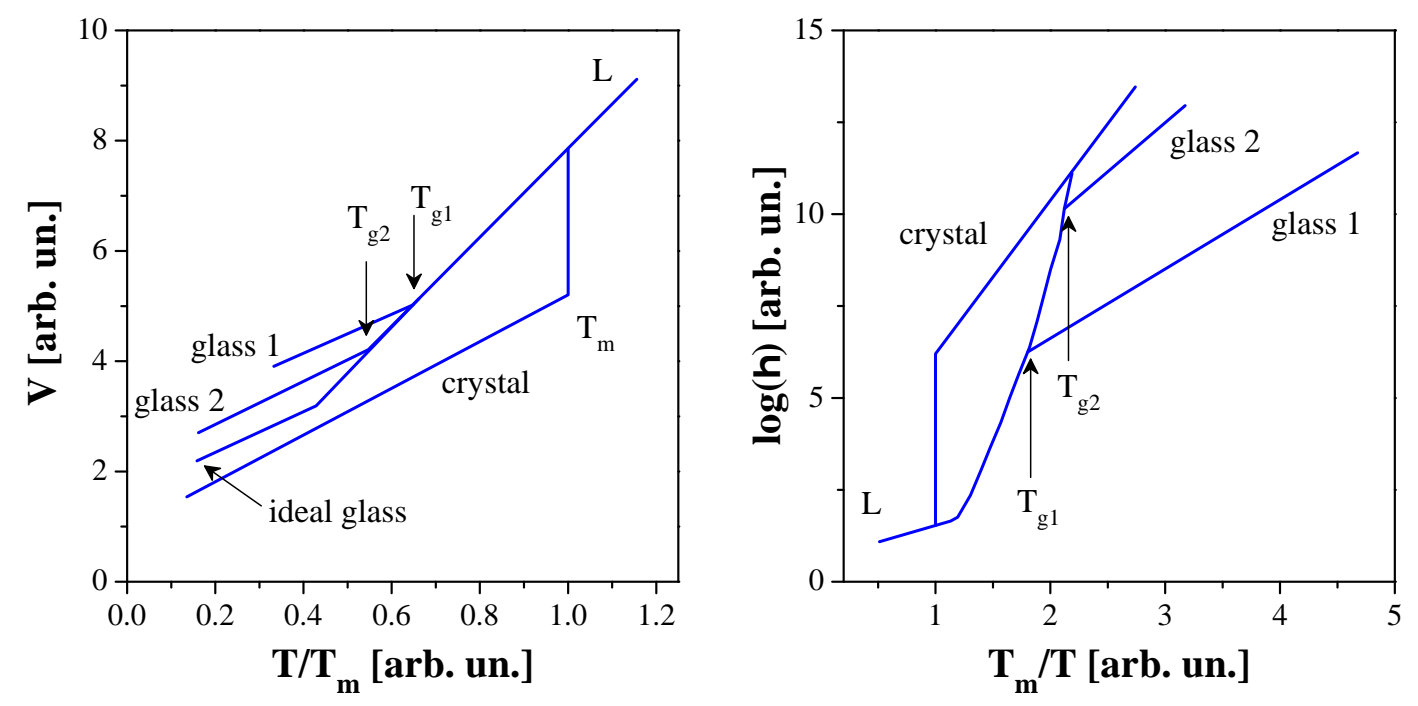

Figure 2.1. Schematic temperature dependence of the volume $V$ and viscosity $\eta$ of a glassy material [31.

transition was first confirmed by Chen and Turnbull [33] and Chen and Goldstein [32] for the rapidly quenched $\mathrm{Au}-\mathrm{Si}$ and $\mathrm{Pd}-\mathrm{Cu}-\mathrm{Si}$ amorphous alloys.

The second definition of glass takes origin from the observation of Cargill [30] about the similarity between the experimentally determined radial distribution function (RDF) of a glass and the theoretical RDF of a dense random packing (DRP) model proposed by 


\section{Bernal $11(1)$}

The structure of a glass at an atomistic level is a direct consequence of how the liquid is cooled down below $T_{m}$ and the DRP of such a disordered materials can be easily distinguished from the structure of a crystalline one. In fact, in crystalline structures, the local environment takes only few discrete patterns and the structural rearrangement during the cooling of the melt occurs only at the interface liquid-solid accompanied by a reduction of the system free energy at each locality and leaving a topologically unfrustrated structure behind the moving interface. On the contrary, the atomic rearrangement in a liquid structure which is necessary to minimize the energy of one atom during undercooling is often incompatible with the rearrangement around the next atom, resulting in a topologically frustrated structure. Due to frustration the configurational entropy of a glass is smaller than that of a liquid since the energy is at least locally minimized, but usually higher than that of a crystal. Thus, a change in entropy as large as the entropy of melting (about $k_{B} \ln (2)$ [53]) must occur at the glass transition.

But, why does a liquid not crystallize upon undercooling but, instead, undergo topological rearrangements and form a glass? On the basis of the standard nucleation theory (see chapter 3.1) it must be concluded that the homogeneous nucleation mechanism, responsible for the formation of the solid phase, must be somehow hampered by a large energy barrier. To explain this conclusion, Frank showed in 1952 61 that, as a metallic liquid is undercooled, local structures develop in the liquid phase (icosahedral shortrange order, ISRO) which, being incompatible with the long-range periodicity of the crystalline phase, creates a barrier to crystallization. Several experimental studies have supported this model, but none provided direct proof of it until Kelton and collaborators in 2003 [88] succeeded in giving the first direct experimental confirmation of Frank's hypothesis demonstrating the connection between the local order of the liquid and the nucleation barrier upon undercooling of a metallic melt.

\subsubsection{Phenomenological Conditions for Glass Formation}

Independently from the phenomenological and/or microscopic definitions of glass, which have been given in the previous section, several experimental conditions determine the glass formation of an alloy of a certain chemical composition.

Since bulk metallic amorphous alloys have been obtained exclusively for limited ranges of composition, it may be thought that glass formation requires very special circumstances as, for example, a specific chemical reaction among the constituent elements or a certain

\footnotetext{
(1) The dense random packing is considered to be the first realistic model of liquid structure. The model demonstrates that an aperiodic structure with a physical density comparable to that of a crystal can exist by physically packing many small spheres inside a large spherical space. Bernal then studied the local topology of several atomic configurations and found clusters and local coordinations which cannot be seen in typical crystalline structures. The most celebrated example of such a cluster is the icosahedron [11].
} 
stoichiometry for the formation of a specific crystallographic structure. That does not seem the case since glass formation is observed in every class of material (insulators, semiconductors, metals and ionic solids) and, hence, points to be a general phenomenon "which can happen to any system as long as sufficient undercooling is achieved" [160. This is because the crystallization of a liquid is a kinetic process which is depressed if the rate of cooling is fast enough to freeze in the liquid to a glassy state. Therefore the relevant question to be asked regarding the glass formation is not whether the specific system forms a glass or not, but as pointed out by Turnbull "what is the critical cooling rate necessary to circumvent the crystallization and vitrify that particular system" $[160$. Turnbull suggested then that the critical cooling rate for glass formation could be reasonably estimated by using the standard nucleation theory. The nucleation rate $I$ of crystalline particles in a liquid which is undercooled below the melting temperature $T_{m}$ by $\Delta T=\left(1-T / T_{m}\right)$ is given by:

$$
I=a D \exp \left(-\frac{\Delta E}{k_{B} T}\right)
$$

where $a$ is a constant, $D$ is the diffusivity and $\Delta E$ is the activation energy for nucleation which is proportional to the cubic power of the liquid-solid interfacial tension and inversely proportional to $(\Delta T)^{2}$ (for a more detailed description of the nucleation and growth theory, see section 3.1). $I$ has a maximum $I_{M}$ at a temperature between $T_{m}$ and $T_{g}$ and the density of the crystalline nuclei is then determined by the product of this maximum nucleation rate $I_{M}$ and the cooling time $\tau_{c}$, which is the inverse of the cooling rate.

This result has two important implications: first, if $\tau_{c}$ is small enough (high cooling rates), the density of the nuclei can be kept low and the alloy remains amorphous; second, it is often difficult to eliminate entirely the nucleation, such that the practical definition of a glass must allow for the possibility that it contains a small number of crystalline nuclei. It is common to define the theoretical boundary which differentiates a glass from a partially crystalline phase by the volume fraction of the crystalline particles. This boundary is arbitrarily set equal to $10^{-6}$, but experimentally a volume fraction of 0.5 is more often used $\left({ }^{(3)}\right.$. The presence of crystalline particles of a second phase embedded

\footnotetext{
${ }^{(2)}$ For instance, since the critical cooling rate for glass formation in pure metallic elements is theoretically calculated to be about $10^{10} \mathrm{~K} / \mathrm{s}$ while the cooling rate of the regular liquid quenching methods (splat cooling, melt spinning, etc.) is estimated to be $10^{5}-10^{6} \mathrm{~K} / \mathrm{s}$, it is evident why amorphous pure metallic elements cannot be produced by liquid quenching but only by vapor deposition which has apparently higher cooling rates.

${ }^{(3)}$ A precise definition of the critical volume fraction does not have much practical significance since it depends strongly upon temperature and time. However if one chooses one definition and plots the time $t$ that a system needs to reach the defined boundary at any temperature $T$, one obtains the so-called time-temperature-transformation diagram which is a powerful mean to determine important information concerning the glassy state of an alloy: critical cooling rate, thermal stability, etc. (see for example sections 3.1 and 3.1 .2 .
} 
in the original amorphous matrix is the prerequisite to obtaining the so-called in-situ formed composite materials (or intrinsic composite) which are the basis of this thesis and will be introduced in section 2.3 .

Determining under which undercooling conditions (critical cooling rate) an alloy forms a glass or rather a partially crystalline material and what controls the so-called glassforming ability (GFA) (or glass-forming tendency [45]) of an alloy which is undercooled with a "regular" quenching method (cooling rates of the order of $10^{5}-10^{6} \mathrm{~K} / \mathrm{s}$ ) is of fundamental importance for the preparation of an intrinsic composite and for the understanding of its microstructural properties. Many attempts to answering to those questions are empirical or phenomenological, while several others are theoretical approaches pointing to the explanation of glass formation [53. The most important of them have been proposed during the last years mainly by Duwez, Giessen, and Cahn:

- The GFA of an alloy is often higher for those compositions which are closer to the eutectic point ${ }^{(4)}$ of the phase diagram of the system 51 . This is intuitively reasonable because the deep depression of the melting temperature $T_{m}$ at the eutectic composition implies that a liquid state is more stable compared to a pure element or to any other point of the diagram. Moreover the undercooling $\Delta T$ decreases as $T_{m}$ becomes closer to the solidification line and, hence, the nucleation rate $I$ remains low favoring the glass formation.

- Giessen 65 used two parameters to map out the regions of easy GFA of an alloy: the heat of mixing $\Delta H_{m}$ and the atomic size ratio of the constituent elements. In a binary alloy $\mathrm{A}-\mathrm{B}$, a glass can be formed if the size ratio $r_{B} / r_{A}$ is either smaller than 0.88 or larger than 1.12 , and $\Delta H_{m}$ is reasonably negative 130 .

- The GFA of a glass can be understood making use of the thermodynamic concept of $T_{0}$ : the temperature at which a liquid and a single-phase solid of the same composition have an equal free energy [27]. Therefore, $T_{0}$ is the melting or freezing temperature of an alloy in the case no phase separation takes place and it is represented in the phase diagram by a line between the liquidus and solidus lines (see figure 2.2. During rapid cooling from the melt, if the velocity of the solidification front is very fast, the composition of the solid becomes almost identical to that of the liquid. In such a case, the solidification temperature is not represented by the liquidus temperature $T_{l}$ but by $T_{0}$ and, once the undercooled liquid reaches it, the solidification kinetic is similar to that of a pure element. Therefore, if the diffusivity at $T_{0}$ is not very low, the solidification kinetics below $T_{0}$ are rather fast and usually

\footnotetext{
${ }^{(4)}$ Eutectic (eutēktos: eu- + têktos, from têkein: to melt, which means easy melted) is the point in the phase diagram of a two (or more) elements at which the liquidus line is at the relative lowest temperature and three (or more) phases coexist: the liquid phase and two (or more) solid ones.
} 
massive crystallization occurs, resulting in a fine-grain supersaturated solid solution. But, if $T_{0}$ is very low, or does not exist (as shown in figure 2.2 for a certain range of compositions by a shaded region), the glassy phase is ultimately stable against the solid solution and, if phase separation does not take place, the glass can more readily be formed.

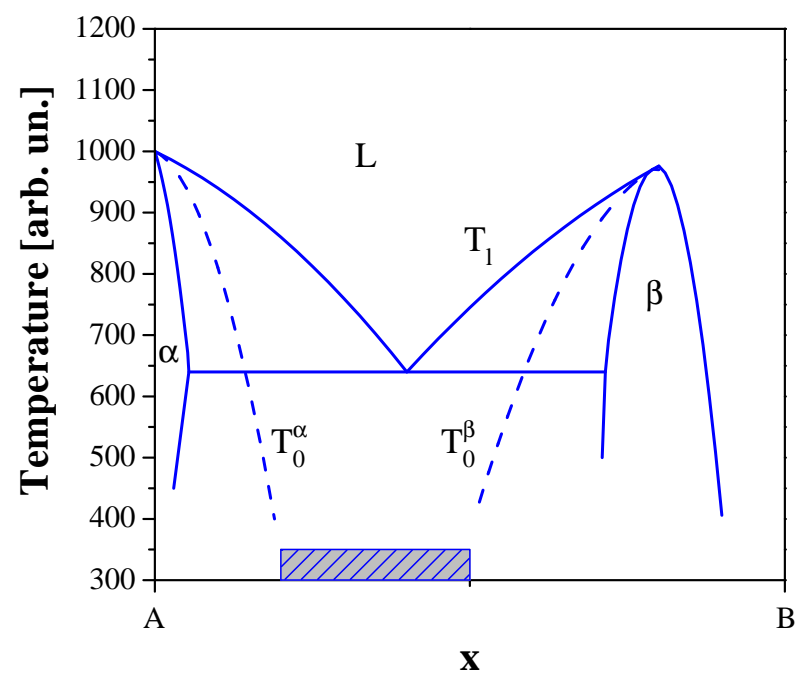

Figure 2.2. Schematic phase diagram of an eutectic binary alloy with $T_{0}$ lines. $\beta$ is an intermetallic compound. The shaded region corresponds to the glass-forming composition.

An approach for determining the glass forming ability of a system which is of considerable practical importance was proposed by Inoue on the basis of metallurgical and empirical considerations and of the different approaches listed above [79]. Inoue suggested the following three rules for the glass forming ability of an alloy:

(i) A good metallic glass former is a multicomponent system with more than three elements. In fact, the critical radius of a crystalline nucleus increases in a multicomponent system making therefore more difficult the nucleation and inhibiting the crystallization.

(ii) The elements forming the alloy must have a relative ratio of the atomic radii larger than $12 \%$. In this way dense packing of different elements becomes more probable and atomic rearrangement can be hampered.

(iii) The constituents must have a large negative heat of mixing. A negative heat of mixing is an indication of the tendency of two chemical species to combine and to form an intermetallic compound. On the contrary, if the enthalpy of mixing is positive the tendency of mutual avoidance is enhanced and the alloy may show phase separation. 
In summary the use of a multicomponent alloy which satisfies Inoue's rules should have a large glass-forming ability and critical cooling rates of the order of magnitude of standard quenching methods. Those methods are the argument of the next section.

\subsection{Preparation Methods of Metallic Glasses}

All three phases of matter: vapor, liquid and solid, have been successfully employed during the last 80 years in the preparation of metallic glasses. Several different techniques were therefore developed for this scope: melt quenching, amorphization by ball milling, thermal deposition, chemical decomposition, reaction amorphization, irradiation, solid-state diffusional amorphization, and many more, but only quenching from the vapor and from the liquid phase (and, in same cases, ball milling) have attracted a wide commercial and scientific interest due to their effectiveness and simplicity in achieving the amorphization of many metallic compositions.

As previously seen, the amorphous phase is thermodynamically less stable than the corresponding crystalline one and shows therefore the tendency to relax or to transform into progressively more stable states. For this reason the basic condition of obtaining amorphous materials is rapid transition into the solid state in such a way that the excess free energy of the amorphous phase (compared to the crystalline one) can be "frozen in" by fast quenching from the melt or by vapor deposition on a cold substrate.

For the preparation of $\mathrm{Nd}_{60} \mathrm{Fe}_{30} \mathrm{Al}_{10}$ bulk samples and thin films both methods: quenching from the melt (see section 2.2.1) and deposition from the vapor (see section 2.2.2 have been employed.

\subsubsection{Melt Quenching}

An essential requirement for the formation of a glass by the quenching from the melt (melt or liquid quenching) is that the cooling must be sufficiently fast and, hence, the cooling rate high enough to exclude crystal nucleation-and-growth and to achieve a solidification characterized by a continuous undercooling of the melt. Several techniques have emerged for this scope during the last 20 years and all of them solidified the molten alloy by bringing the melt in contact with a cold surface.

Three are the preparation methods used to produce $\mathrm{Nd}_{60} \mathrm{Fe}_{30} \mathrm{Al}_{10}$ bulk samples presented in this work: arc melting, splat quenching, and copper mold casting. Each method differs from the others by the technological solutions adopted for the rapid quenching of the melt and, hence, by a characteristic cooling rate which varies from one technique to the other of several orders of magnitude. Beyond the differences, however, all the three methods which are described in the next sections present a common feature: the necessity to work with a master ingot. Such ingots, prepared by induction melting of the pure met- 
als in a so-called "silver boat", determine the chemical composition of the final samples and, sometimes, their quality.

\subsubsection{1 "Silver Boat" Induction Melter}

The master ingots are prepared by mixing the three metals: Nd, Fe, and $\mathrm{Al}$ in the form of small high purity lumps with the appropriate weights determined by the atomic percentage 60:30:10. The lumps are melted together in a so-called silver boat which is constituted of a water-cooled copper tube which is especially formed to support up to three samples (see figure 2.3). The set-up is closed in a quartz tube which can be evacuated down to
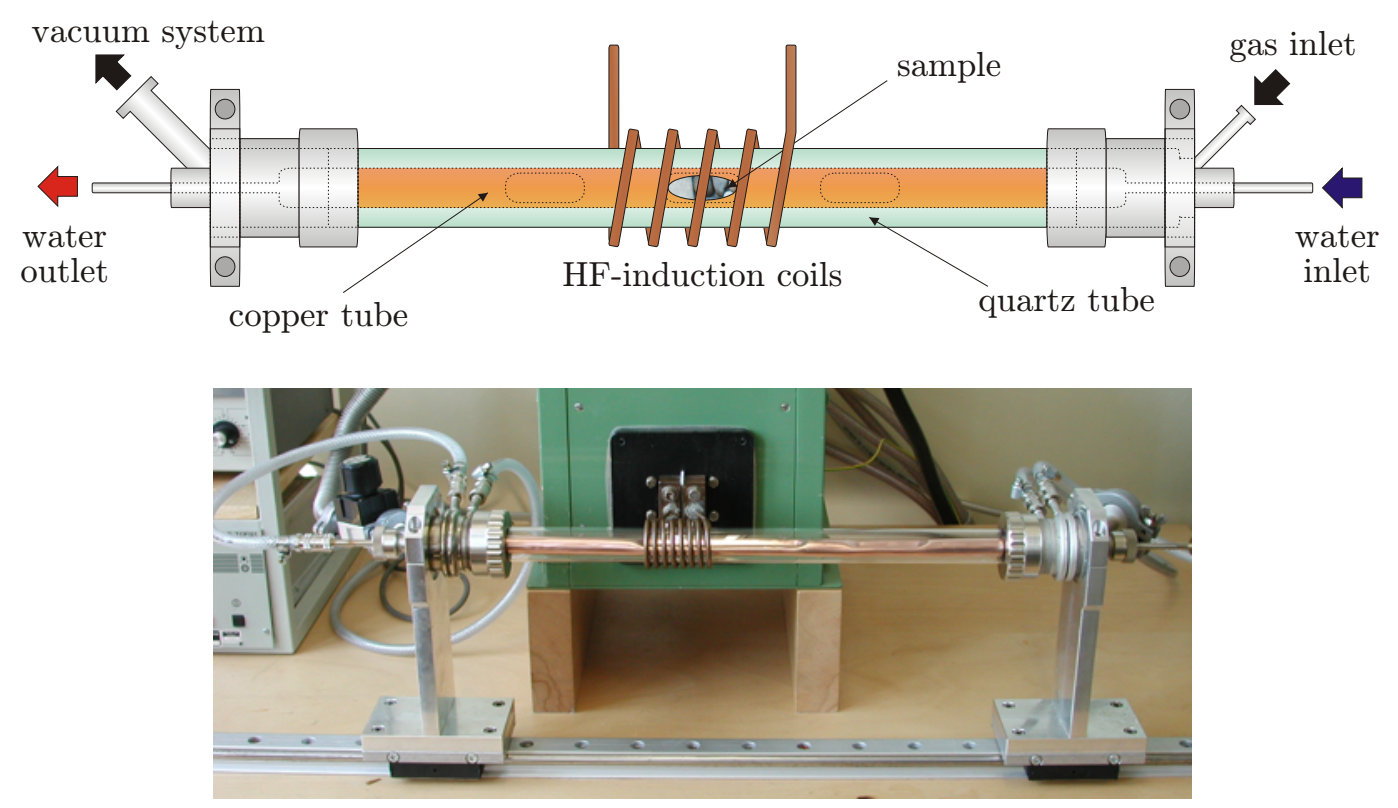

Figure 2.3. (Top) Schematic drawing of a water-cooled "silver boat". (Bottom) The "silver boat" used for the preparation of the $\mathrm{Nd}_{60} \mathrm{Fe}_{30} \mathrm{Al}_{10}$ master ingots.

a pressure of about $10^{-6}$ mbar or filled with inert gas (usually 800 mbar of high purity Ar gas) to prevent the oxidation and the evaporation of the sample during melting. A radio-frequency generator is used to power a coil wound around the quartz tube which produces an alternating magnetic induction in proximity of the sample. A conductive material reacts to such variable induction producing eddy currents (Foucault currents) which dissipate the energy of the external field by Joule effect and, hence, heat up the sample. If the electrical resistivity of the material is not too high, the power dissipation ( $W=f^{2} / R$, where $f$ is the induced e.m.f. and $R$ is the materials resistance) is large enough to melt the sample which can be afterwards cooled down through the watercooled copper tube. Each master ingot is molten 4 to 5 times for at least 30 minutes to insure its homogenization.

Due to the strong tendency of the rare-earth elements to oxidize, each step of the alloy preparation: mechanical cleaning of lumps, their weighting, and their loading in the 
silver boat, was done under protective atmosphere (99.999\% pure Ar gas) in a glove box (not shown).

\subsubsection{Arc Melting}

The use of the silver boat has mainly two advantages: controlled atmosphere to prevent oxidation and the possibility to produce large samples, but it shows three important disadvantages: sometimes the samples are inhomogeneous, the cooling rates are quite low, specimens which are too large are difficult to characterize. For these reasons the original master alloy is crushed in small pieces and a second technique is utilized to remelt and to homogenize the pieces: arc melting.

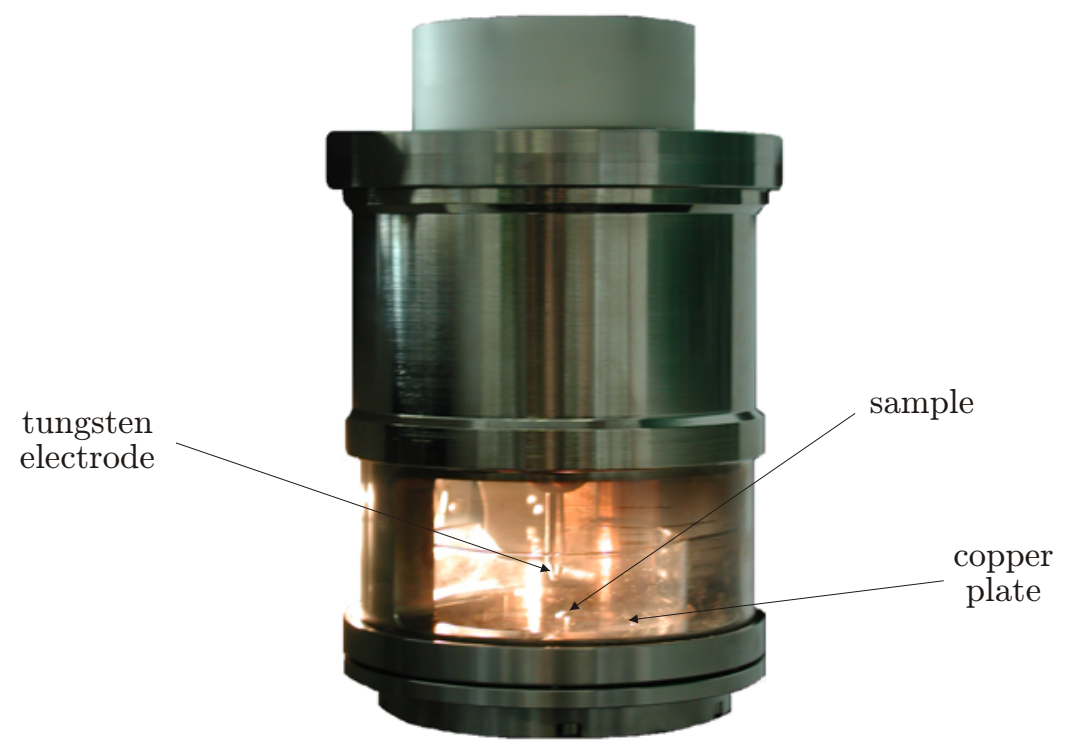

Figure 2.4. Picture of a MAM 1 arc melter (Otto Gmbh) used for the melting and further homogenization of the $\mathrm{Nd}_{60} \mathrm{Fe}_{30} \mathrm{Al}_{10}$ bulk samples.

An arc melter (see figure 2.4) uses a continuous arc discharge to provide current flow between a tungsten electrode and a water-chilled copper plate which is used as electrical ground. As soon as the electrode is positioned in the vicinity of a sample a current of the order of 100 A flows through it and temperatures as high as $3000 \mathrm{~K}$ can be reached. The sample can be therefore easily melted and then cooled down by the copper plate with a rate of some K/s. Samples, electrode and ground plate are closed in a quartz chamber which can be evacuated or filled with high purity inert Ar atmosphere to prevent contamination during the melting or oxygen-induced crystallization.

Due to the surface tension of the melt, specimens prepared by arc melting are small spheres which are suitable for most of the characterization methods and for the preparation of rapid cooled samples by splat quenching (see section 2.2.1.4. 
The specimens prepared by arc melting will be called in the following master alloy (or bulk master alloy).

\subsubsection{Copper Mold Casting}

The most widely used method for producing bulk amorphous alloys with a relatively high cooling rate is the chill-block liquid quenching method (or copper mold casting). The principle used is the direct rapid solidification of the melt through its contact with a metal block which quickly extracts heat from the sample. In implementing this technique, various apparatuses have been designed and built but the most used configuration is that presented in figure 2.5 which makes use of a high vacuum chamber and melt injection device originally developed for melt-spinning 99,126 .

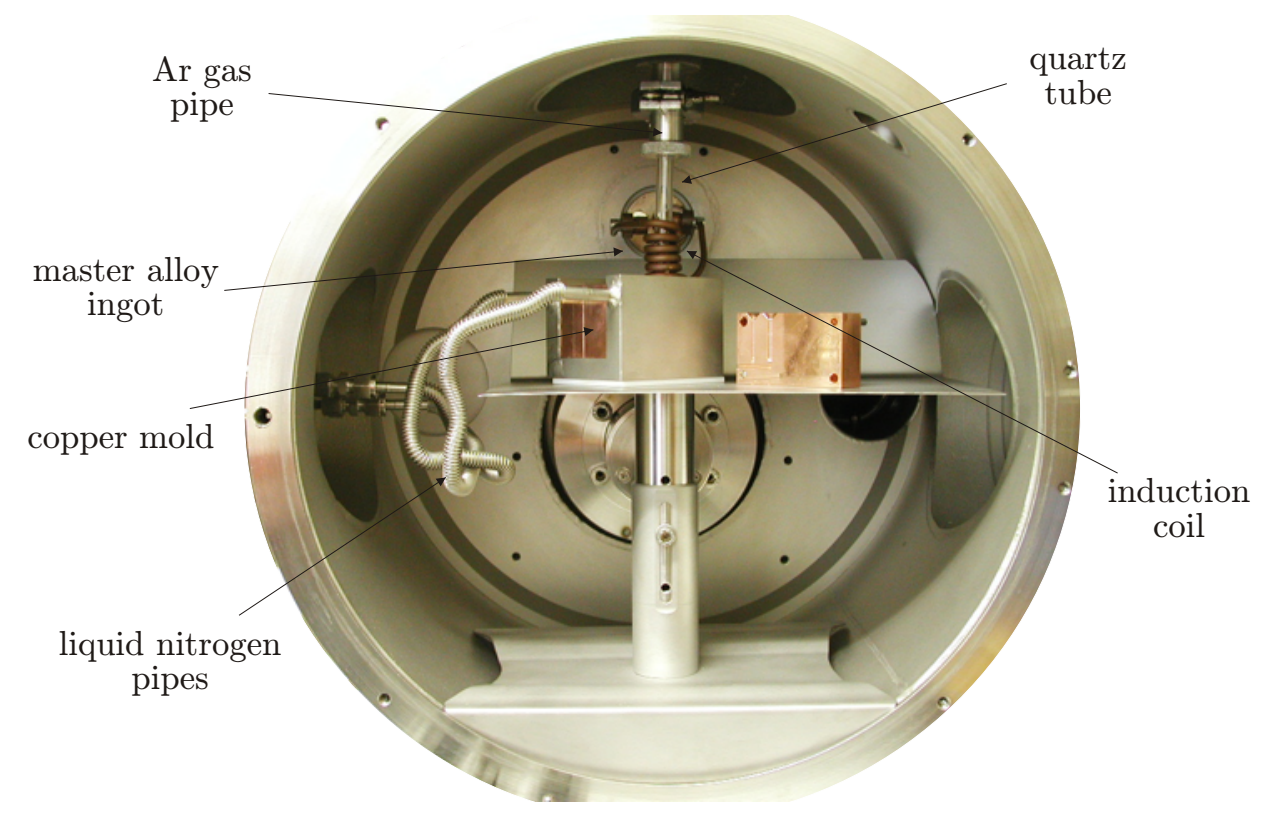

Figure 2.5. Picture of a ultra rapid quencher (Buehler Gmbh) used as copper mold casting device for the preparation of $\mathrm{Nd}_{60} \mathrm{Fe}_{30} \mathrm{Al}_{10}$ bulk samples.

A master ingot prepared by "silver boat" melting is positioned in a quartz tube whose upper side is connected to an external gas cylinder filled with about 900 mbar Ar and whose bottom has a narrow nozzle. The tube is located in the middle of a coil with which the specimen is molted by induction of high frequency Foucault currents. The molten alloy is then ejected through the nozzle in a copper mold by the pressure difference between cylinder and casting chamber which is maintained for this reason at about 600 mbar. To achieve higher cooling rate, the copper block is cooled down to $77 \mathrm{~K}$ by liquid nitrogen flowing through it entering/and leaving the casting chamber by two separate pipes.

Mold-cast samples are cylinders of $3 \mathrm{~mm}$ in diameter and about $25 \mathrm{~mm}$ long (see figure 2.6 which are quenched with a cooling rate of about $10^{2} \mathrm{~K} / \mathrm{s}$ (conventionally accepted as the cooling rate of a standard copper mold casting device). 


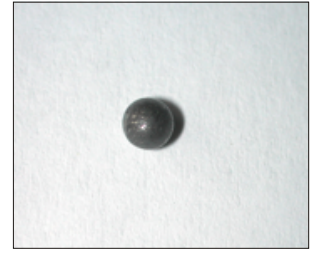

master alloy

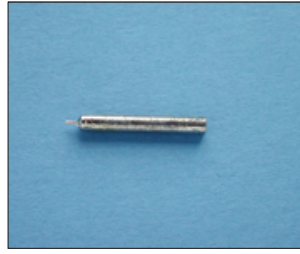

bulk

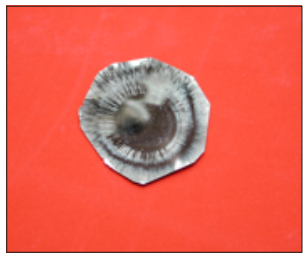

splat

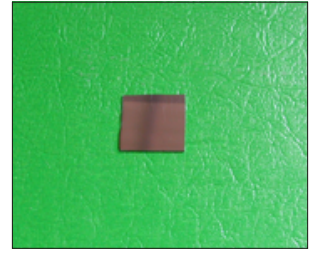

thin film

Figure 2.6. A master alloy, a mold-cast, a splat-cooled sample and a thin film of nominal composition $\mathrm{Nd}_{60} \mathrm{Fe}_{30} \mathrm{Al}_{10}$ prepared by arc melting, copper mold casting, splat quenching and electron beam co-evaporation, respectively.

\subsubsection{Splat Quenching}

A second technique well known method for the preparation of vitreous solids by melt cooling is the so-called splat quenching technique. In a splat quencher (see figure 2.7) a drop of liquid metal is obtained by melting in a induction coil a small piece of the master alloy previously prepared by arc melting (see section 2.2.1.2). Beyond increasing

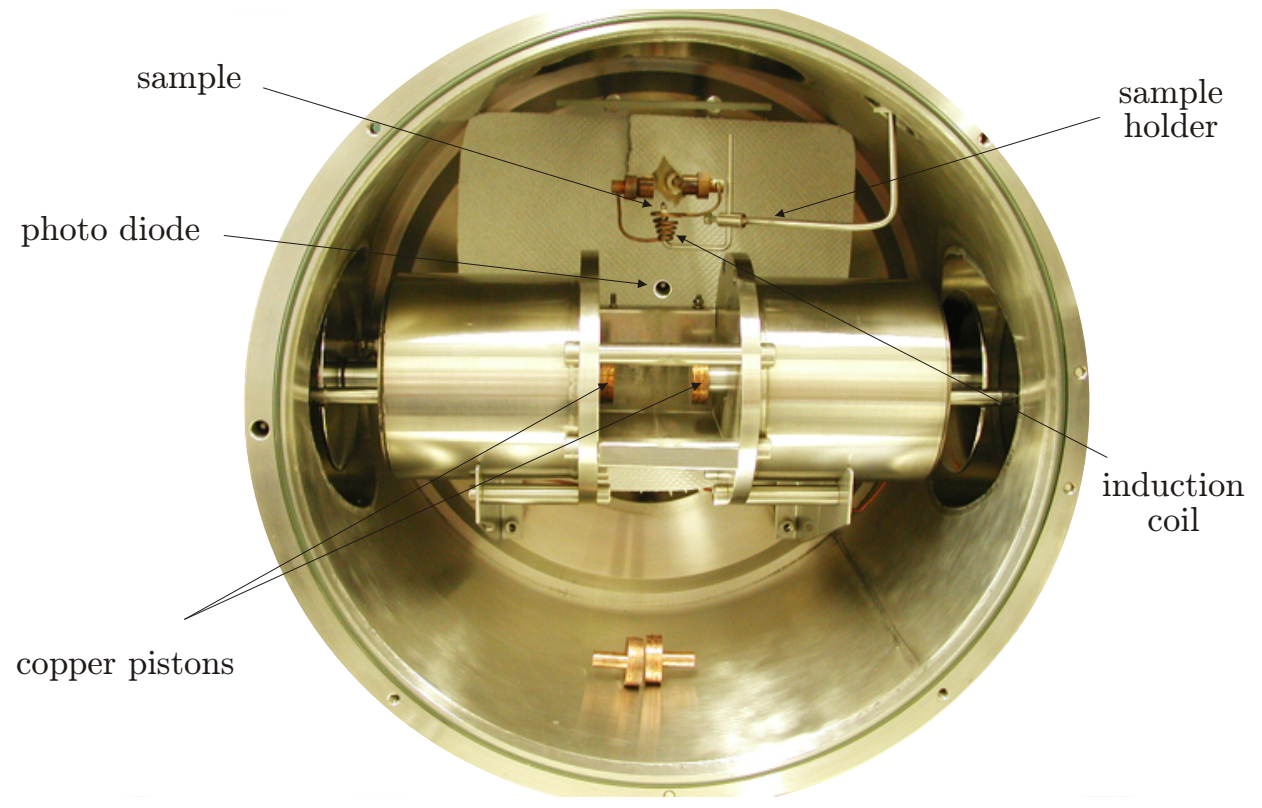

Figure 2.7. Picture of a splat quenching device (Buehler Gmbh) used for the preparation of $\mathrm{Nd}_{60} \mathrm{Fe}_{30} \mathrm{Al}_{10}$ splat foils.

the temperature of the sample by energy dissipation, the induced eddy currents produce a magnetic moment which couples the external induction field and, hence, a force that can balance the gravity force if the sample mass is not too large. Thus the molten specimen can levitate in the middle of the induction coil without being in contact with any holder and, as soon as the current generator connected to the coil is switched off, it falls down by gravity and is caught between two rapidly moving pistons triggered by a light diode. The sample preparation process occurs in an Ar filled chamber which is repeatedly evacuated 
and flushed to achieve an oxygen-free atmosphere and prevent contamination. Typical samples obtained by splat quenching are thin discs (or foils) about $20 \mathrm{~mm}$ across and $30 \mu \mathrm{m}$ thick. The cooling rate is typically $10^{6} \mathrm{~K} / \mathrm{s}$ and, hence, higher than that achievable by copper mold casting. A splat-cooled foils (for clarity also called splat in the following of this work) is shown in figure 2.6.

\subsubsection{Vapor Deposition}

Among the different deposition methods usually utilized in the preparation of thin amorphous films: thermal evaporation, sputtering, glow-discharge decomposition, chemical vapor decomposition (CVD), and several others, evaporation is a well established technique for the deposition in vacuum of atoms from one or more heated sources onto a cold substrate. Thermal evaporation is normally achieved by melting the material to be deposited though resistance, electron-beam or laser heating under the common essential requirement that the vapor pressure is sufficient to permit the atoms to leave the molten source.

Although the atomistic process of deposition is not yet exactly known, it is commonly suggested that each atom which condensates on a substrate leaves the vapor state for the solid one in a very short time giving the possibility to achieve in this way very high quenching rates $[53$. Therefore the primary cooling is indeed quite rapid and a quenching rate of $\sim 10^{10} \mathrm{~K} / \mathrm{s}$ is generally accepted as its order of magnitude. However, the surface of an evaporated sample can stay at relatively high temperatures for a long period of time permitting the deposited atoms to relax or to migrate by diffusion. Thus the effective

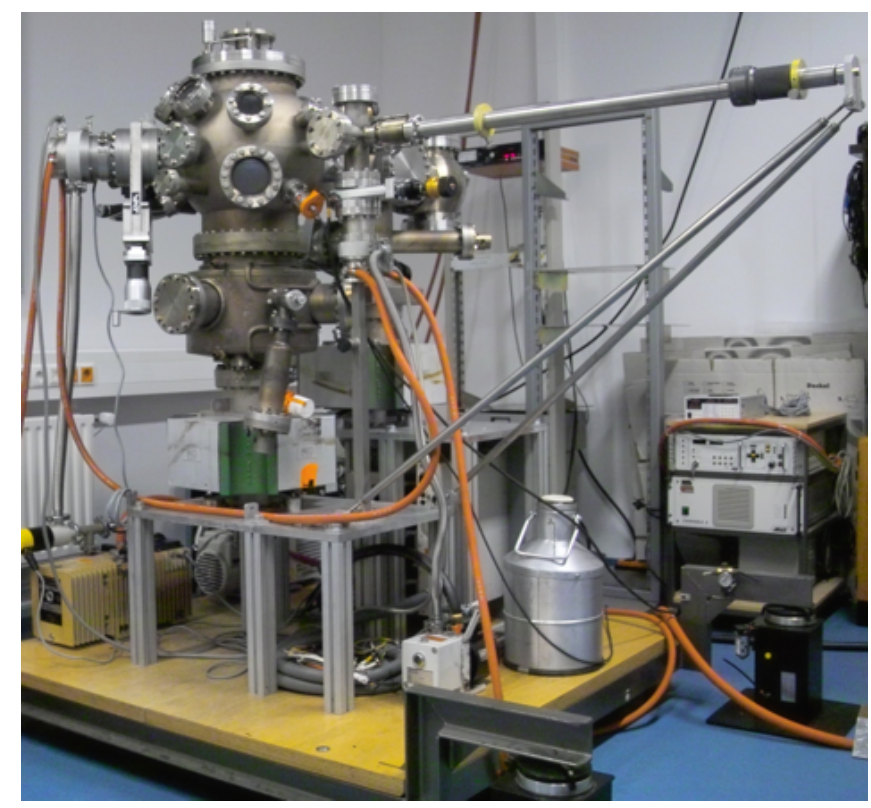

Figure 2.8. UHV chamber employed for the co-evaporation of $\mathrm{Nd}, \mathrm{Fe}$ and $\mathrm{Al}$ in the preparation of $\mathrm{Nd}_{60} \mathrm{Fe}_{30} \mathrm{Al}_{10}$ thin films. 
quenching rate may be not so high as intuitively thought and, in some circumstances (deposition by sputtering, long deposition time, thick samples, etc.), it may be comparable to that of liquid quenching. Although melt quenching and vapor deposition may give rise to comparable cooling rates, an important difference between the two methods exists and must be highlighted: the deposition from the vapor phase bypasses always the liquid state implying that an alloy can be made amorphous easier by vapor deposition than by liquid quenching even if the elementary components show strong positive heat of mixing $\Delta H_{m}$ and tend to phase-separate in the liquid state. In fact the negative value of $\Delta H_{m}$ is apparently a required condition only for glass formation by liquid quenching but not for vapor deposition (see for instance the results proposed by Chien and Unruh on $\mathrm{Fe}-\mathrm{Ag}$ films obtained by ion sputtering [36]).

The method used for the preparation of the $\mathrm{Nd}_{60} \mathrm{Fe}_{30} \mathrm{Al}_{10}$ thin films is briefly presented in the next section.

\subsubsection{Electron-Beam Evaporation}

$\mathrm{Nd}_{60} \mathrm{Fe}_{30} \mathrm{Al}_{10}$ thin films were prepared in an ultra high vacuum (UHV) chamber by electron-beam co-evaporation of the three pure elementary metals: $\mathrm{Nd}, \mathrm{Fe}$, and $\mathrm{Al}$, in order to better control the deposition rates of each element and, hence, the final chemical composition of the films. Figure 2.8 shows the employed UHV chamber while the geometry of its inside is schematically reported in figure 2.9 .

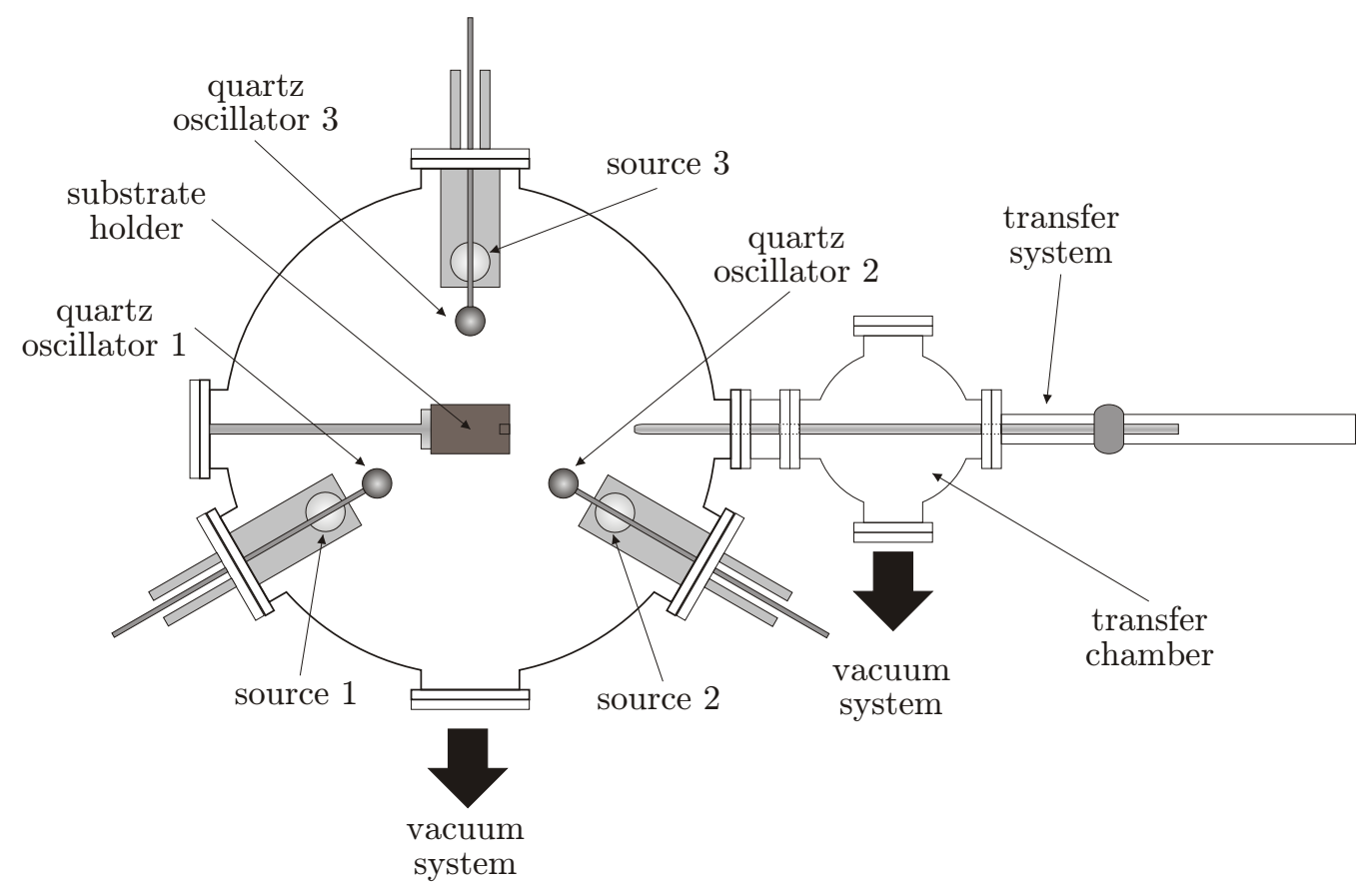

Figure 2.9. Schematic drawing of UHV chamber used for electron beam co-evaporation and of the transfer system employed for loading/unloading of the deposited substrate. 
In the co-evaporation method, each component which forms the final film is situated in a different holder and is melted independently from the other by an electron beam produced by an heated filament. Each molten metal is then evaporated and deposited on a substrate situated above the crucibles. The chemical composition of the films depends on the evaporation rate of each elementary component which is constantly monitored (and corrected due to geometrical factors) by a quartz balance (see figure 2.9.

Thin films of chemical composition $\mathrm{Nd}_{60} \mathrm{Fe}_{30} \mathrm{Al}_{10}$ were evaporated on thermally oxidized Si substrate achieving a specimen thickness of about $50 \mathrm{~nm}$.

\subsection{Intrinsic Composites}

Although many techniques may be employed to achieve high cooling rates, not all chemical compositions form a glass and, on the contrary, not all solidification methods can achieve fully amorphization of a certain composition. The conclusion which arises from section 2.1 and 2.2 is therefore that the preparation of a homogeneous and fully amorphous specimen depends on the chemical composition of the alloy and on the cooling technique. This two aspects determine the main experimental limitations to the formation of a glass.

Two scenarios are possible in the case that the previous conditions are not fully satisfied during the alloy solidification. The final sample consists of several non-crystalline phases of different compositions (phase separation) as a result of immiscibility phenomena in the liquidus state or crystalline clusters (precipitates) nucleate and growth forming a complex heterogeneous structure which consists of grains of a stable crystalline phase embedded in a remnant disordered matrix (composite material). In both cases, phase separation or precipitates formation modifies the microstructure and the "texture" of the glass on a nanometer scale influencing substantially physical and chemical properties of the resulting inhomogeneous specimen [53].

What is in reality a composite material? A composite material is a system consisting of two or more physically and/or chemically distinct, suitably arranged or distributed phases, with an interface separating them whose characteristics are not depicted by any of the components acting alone. Most commonly, composite materials have a bulk phase, which is continuous, called the matrix, and two dispersed, non-continuous, phases called respectively reinforcement and interface. Those materials which are composite on a macroscopic scale are commonly defined extrinsic composites while if the composition occurs on a microscopic scale and in-situ, for example upon cooling of the melt, the new material is then called intrinsic or in-situ composite.

The concept of composite materials is old and well-known: combine different materials

\footnotetext{
${ }^{(5)}$ It has been also demonstrated that metastable phase separation can play an important as precursor in crystallization of glasses 179 and, hence, the formation of crystalline nuclei and phase separation are two processes which may strongly interact.
} 
to produce a new material with performance unattainable by the individual constituents. An example is adding straw to mud for building stronger walls or carbon black in rubber, steel rods in concrete, cement/asphalt mixed with sand, fiberglass in resin, etc. while the most important examples in nature are cellulose fibers in a lignin matrix (wood), collagen fibers in an apatite matrix (bone), many more.

The singular physical properties of composite materials and, in particular, of the intrinsic composites: corrosion and oxidation resistance, mechanical or magnetic features [40], depend on the properties of the matrix, of the precipitates and moreover on the nature and morphology of the interface coupling them. This is mainly due to the fact that the interface is usually a large bounding surface which shows physical, mechanical, and chemical discontinuities and guarantees the necessary strong adhesion between precipitates and matrix. Some examples of the improved physical properties which have made intrinsic composites promising candidates for technological and engineering applications are presented in the next section.

\subsubsection{Physical properties of Intrinsic Composites}

\subsubsection{Mechanical properties}

In recent years many metallic glass forming alloys have been developed. Among them bulk metallic glasses of composition $\mathrm{La}-\mathrm{Al}-\mathrm{Ni}, \mathrm{Zr}-\mathrm{Al}-\mathrm{Cu}-\mathrm{Ni}(-\mathrm{Nb}), \mathrm{Zr}-\mathrm{Ti}-\mathrm{Cu}-\mathrm{Ni}(-\mathrm{Be})$, could be successfully cast with cooling rates of less than $100 \mathrm{~K} / \mathrm{s}$. Bulk metallic glasses have high yield strength and a high elastic strain limit combined with corrosion resistance, and relatively high fracture toughness (see figure 2.10. However, the applications of such

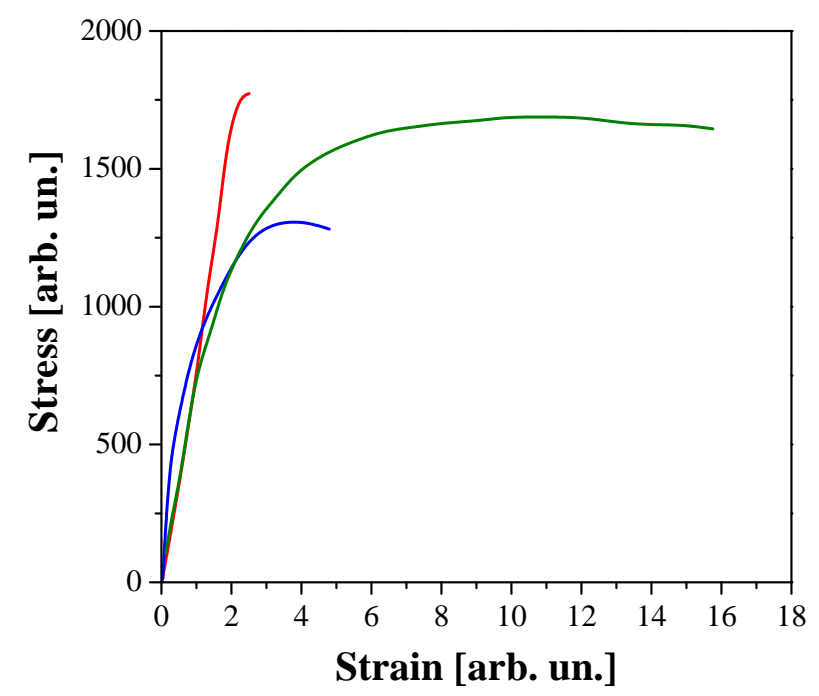

Figure 2.10. Compressive strain-stress curves of monolithic $\mathrm{Zr}_{57} \mathrm{Nb}_{5} \mathrm{Al}_{10} \mathrm{Cu}_{15.4} \mathrm{Ni}_{12.6}$ Vit106 (-) and Vit106 + W particles composite processed at $1150(-)$ and $1425 \mathrm{~K}(-)$.

materials have been limited by the low plasticity and, hence, their tendency to break 
under a relatively low stress.

To improve the toughness of metallic glasses by hindering propagation of shear bands and encouraging the formation of multiple pinning centers two different approaches have been followed: the first is to introduce foreign particles into the matrix (glass reinforcement) and, hence, to form an extrinsic composite $\left(\mathrm{Cu}_{47} \mathrm{Ti}_{34} \mathrm{Zr}_{11} \mathrm{Ni}_{8}+\mathrm{SiC}\right.$ or $\left.\mathrm{TiC}[38]\right)$ while the second is to guarantee a strong interface reinforcement-matrix by in-situ partially crystallization of the amorphous matrix upon cooling or upon subsequent heating $\left(\mathrm{Zr}_{41.2} \mathrm{Ti}_{13.8} \mathrm{Cu}_{12.5} \mathrm{Ni}_{10} \mathrm{Be}_{22.5} \mid 73\right] ; \mathrm{Zr}_{57} \mathrm{Nb}_{5} \mathrm{Al}_{10} \mathrm{Cu}_{15.4} \mathrm{Ni}_{12.6}+\mathrm{WC}, \mathrm{W}$, or Ta [39]). Size and volume fraction of the reinforcement crystals can be controlled by varying composition, processing time, and temperature as well as the mechanical properties which are shown in figure 2.10 .

The improved stress-strain curves of figure 2.10 are only one of many examples of engineering composites which are nowadays of high industrial and scientific interest.

\subsubsection{Magnetic properties}

In the last 20 years the interest for complex magnetic materials showing outstanding magnetic properties has been continuously growing. Among all the advanced magnetic materials used today (high permeability $\mathrm{Fe}_{3} \mathrm{Si}$-based Finemet, colossal magnetoresistive $\mathrm{LaCa}-$ Manganites, ferromagnetic nanoparticles FePt or FeCo) the class of the high-coercivity compounds is that probably more closed to the concept of (intrinsic) composite materials. In figure 2.11 is shown the progress in improving the hard magnetic properties of poly-

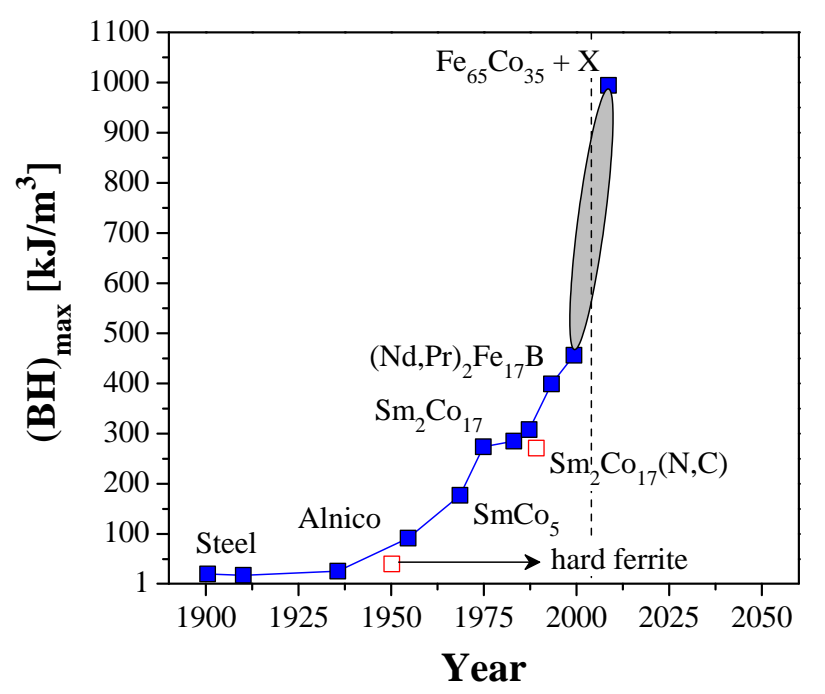

Figure 2.11. Progress in improving $(B H)_{\max }$ over the last century. The shaded region covers hypothetical FeCo-based alloys, which are magnetically hardened by additives X.

crystalline samples during the last century. The major improvement have been obtained in the last 20 years with those magnetic materials (SmCo family and the FeCo alloys) 
composed of two or more phases showing high coercivity values and pinning effects of the main magnetic phase. In particular, it has been demonstrated that the high coercivity of the SmCo-type permanent magnets originates from a complex microstructure consisting of a superposition of a cellular $\left(\mathrm{Sm}_{2}(\mathrm{Co}, \mathrm{Fe})_{17}\right.$ cells and $\mathrm{Sm}(\mathrm{Co}, \mathrm{Fe})_{5}$ cell boundaries $)$ and a lamellar structure (see figure 2.12). The $\mathrm{Sm}_{2} \mathrm{Co}_{17}$ cells are mainly responsible for the large saturation magnetization while the $\mathrm{SmCo}_{5}$ precipitates act as pinning sites for magnetic domains walls whose motion is stopped until the external field is increased above the pinning field $70,140 \mid$.

Such magnetic behavior highlights the importance of the heterogeneous nature of the microstructure in which each component (precipitates, interface and matrix) contributes separately to the overall magnetic characteristics of the compound and it will be recall in the discussion of the magnetic properties of the $\mathrm{Nd}_{60} \mathrm{Fe}_{30} \mathrm{Al}_{10}$ samples.

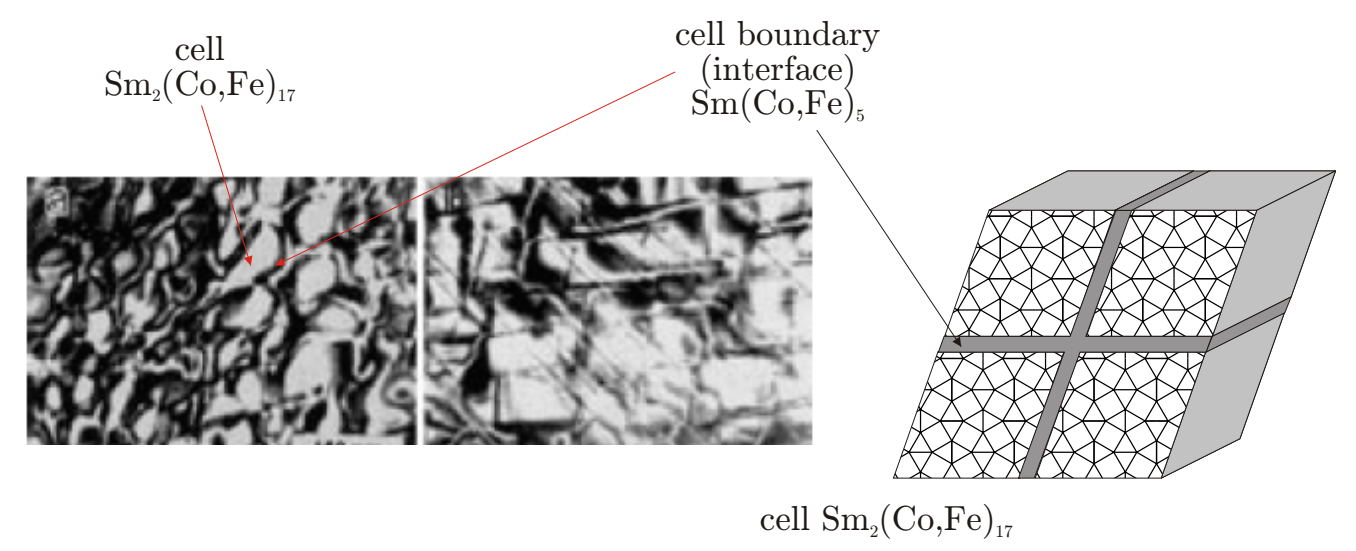

Figure 2.12. Cellular structure in SmCo-type permanent magnets found to be responsible for the hard magnetic behavior and the high values of the coercive field. 


\section{Chapter 3}

\section{Thermal Stability of Intrinsic Composite Materials}

Whenever a system is quenched into a metastable state, the problem of its thermal stability upon thermal treatments or application of external stress must be taken into account due to the strong variation of the microstructure and, hence, of the physical properties which these factors may induce.

Under this point of view intrinsic composites (as defined in chapter 2) are an example of metastable systems that may present different transformation paths towards progressively more stable states. The presence of nanosized crystals embedded in a glassy matrix implies that composite materials may show four broad classes of transformation processes by which the free energy of the system can be lowered: structural relaxation, crystallization, growth, coarsening.

Structural relaxation is the process by which a material relaxes from its initial metastable state towards its internal equilibrium state [4]. In the case of amorphous specimens this process is driven by the considerable strain frozen-in during glass formation [126] and is dominated by atomic diffusion. The equilibrium state of an amorphous material is not however the most stable state since the system can still lower its overall free energy by crystallization. This is the event in which a second phase nucleates from the amorphous matrix and forms crystalline nuclei (precipitates) which can then grow by solid state transformations. Growth processes may affect of course not only the new formed precipitates but also the in-situ nanocrystalline phase present in an intrinsic composite system. The growth is in general driven by concentration gradients between matrix and particles which are frozen-in upon the fast quenching typically employed for the preparation of an intrinsic composite. When the composition of the matrix is nearly at its equilibrium value coarsening occurs 133 and the matrix is no longer a source or a sink of solute and the majority of the mass transport is from small to large particles. Coarsening is driven by the decrease in interfacial energy per unit volume which results from the conversion of numerous small precipitates to a few large ones. 
The first to observe the coarsening process was Ostwald in 1900 and the phenomenon has took for this reason his name: Ostwald ripening [129]. Ostwald ripening can occur in virtually any two-phase system in which there is significant diffusion. From the first experimental evidence of the Ostwald ripening it took about 60 years before coming to a successful theory which could describe it (Lifshitz and Slyozov [108], Wagner [166]) and only nowadays the significant impact of coarsening on how materials can be processed has been fully understood (age hardening of metallic alloys, grain refining, grain growth during recrystallization, and many others).

Due to the importance of the crystallization event in the structural evolution of the $\mathrm{Nd}_{60} \mathrm{Fe}_{30} \mathrm{Al}_{10}$ system, section 3.1 is dedicated to a brief description of the nucleationand-growth problem ${ }^{(1)}$ emphasizing the relevance of the so-called Kissinger's analysis (section 3.1.1) and of the continuous heating transformation diagram (section 3.1.2) in the investigation of this process. A description of the coarsening process and of the theories which permit quantitatively to study it is presented in section 3.2 while the experimental approach used to interpret the results shown in chapter 6 is the main topic of section 3.2.1.

\subsection{Nucleation-and-Growth}

When a single phase system is put into a two-phase metastable state a second phase nucleates $[20$ and grows. Nucleation of the second phase occurs since the energy of the single phase system can be reduced by forming regions of the second phase which will appear as nanometer-sized nuclei. Growth of nuclei proceeds then due to heat diffusion away from the nucleus into the matrix or mass diffusion from the matrix to the nuclei.

Calling $\alpha$ the phase of the matrix and $\beta$ the phase of the forming nucleus in a system composed of $A$ and $B$ atoms $\left(x_{\mathrm{B}}^{0}\right.$ is the concentration of $B$ atoms in the $A-B$ binary system), the formation of a $\beta$ particle within the $\alpha$-phase is allowed in classical thermodynamics only if the work of formation of the nucleus $\left(W_{r}\right)$ is negative. However, it is possible to have local increases in energy $\left(W_{r}>0\right)$, fluctuations, as long as the average change in energy is zero or negative. Thus fluctuations play an important role in the nucleation process and their magnitude can be appreciate determining the value of $W_{r}$ for the so-called critical radius $r^{*}$ which defines the size of a cluster that is in unstable equilibrium with the matrix and that growths or shrinks as consequence of any perturbation in the system. The dependence of the work $W_{r}$ on the radius $r$ is given by the

\footnotetext{
${ }^{(1)}$ For a detailed discussion on how a single phase system transforms into a multiphase material see 68 , 133 and references therein, or 60.126 160.

${ }^{(2)}$ Of course nucleation takes place if the single phase system is placed in a region of the phase diagram in which in equilibrium two phases, $\alpha$ and $\beta$, of different composition coexist. In the case of intrinsic composites that can be achieved, for example, by quenching the melt from a high to a sufficiently low temperature or changing pressure, and in the case of homogeneous fully amorphous materials, by annealing at high temperature (crystallization).
} 
relation 68 :

$$
W_{r}=-\Delta f \cdot \frac{4}{3} \pi r^{3}+E_{\alpha \beta} \cdot r^{2}
$$

where $\Delta f$ is the saving in volume free energy resulting from the formation of a precipitate $\beta$ in the $\alpha$-matrix, $E_{\alpha \beta}$ is the gain in surface energy ${ }^{(3)}$. A schematic representation of $W_{r}$ is reported in figure 3.1 . The critical radius $r^{*}$ for nucleation is at the maximum of the

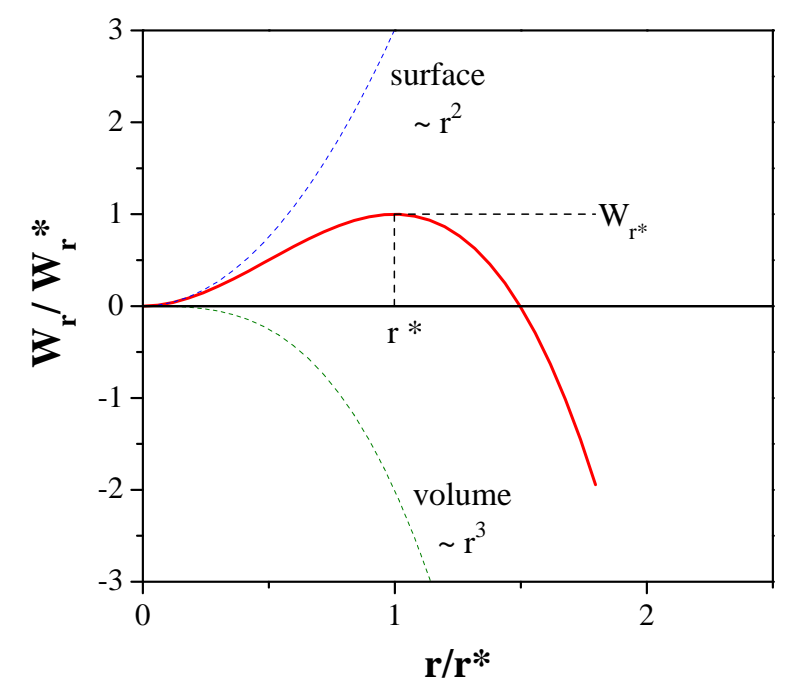

Figure 3.1. Work of formation $W_{r}$ of a cluster of size $r$.

curve, as expected for unstable equilibrium, and its value is determined by the condition

$$
\left.\frac{d W_{r}}{d r}\right|_{r=r^{*}}=-\Delta f \cdot 4 \pi r^{2}+2 E_{\alpha \beta} \cdot r=0
$$

which gives

$$
r^{*}=\frac{E_{\alpha \beta}}{2 \pi \Delta f}
$$

From this relation it is evident that the larger $\Delta f$ (or the smaller $E_{\alpha \beta}$ ) the smaller the critical radius of the nucleus and, hence, more probable its formation. In a single phase material which is cooled from the melt it can be shown that the gain in volume free energy is directly proportional to the undercooling $\Delta T$ defined as 160 :

$$
\Delta T=1-\frac{T}{T_{m}}
$$

where $T_{m}$ is the equilibrium melting temperature of the system, and the critical radius in the solidification from the melt is therefore inversely proportional to $\Delta T$ while the energy barrier $W_{r^{*}}$ to $(\Delta T)^{2}{ }^{2(4)}$,

\footnotetext{
${ }^{(3)}$ In a simple phase material, $\Delta f$ becomes the saving in volume free energy resulting from the solidification of a crystal $\left(\Delta f=f_{\text {liquid }}-f_{\text {solid }}\right)$ and $E_{\alpha \beta}\left(=E_{\mathrm{SL}}\right)$ is the increase in free energy associated with the phase boundary formation.

${ }^{(4)}$ In fact, if we assume that
} 
According to Ratke [133] (and, similarly, to Turnbull [160]) the rate with which the nuclei form (nucleation rate) can be expressed by the relation

$$
I=I_{0} D \cdot \exp \left(-\frac{W_{r^{*}}}{k_{B} T}\right)
$$

which can be rewritten taking into account an Arrhenius law for the diffusion coefficient $D$ as

$$
I=I_{0} D_{0} \cdot \exp \left(-\frac{\Delta E}{k_{B} T}\right) \exp \left(-\frac{W_{r^{*}}}{k_{B} T}\right)
$$

where $\Delta E$ is the activation energy for diffusion. For small $\Delta f$ (small supersaturation of the matrix), or small $\Delta T$ (small undercooling), the nucleation rate is very small but it increases rapidly, if the alloy composition is fixed, upon decreasing the temperature due a change of $\Delta f$ or upon increasing of $\Delta T$. At sufficiently low temperatures, on the contrary, due to the $1 / T$ dependence, the diffusion coefficient becomes again very small and thus a maximum is expected in a intermediate region. The described behavior is observed also in single phase materials for which the undercooling $\Delta T$ controls the nucleation rate $I$.

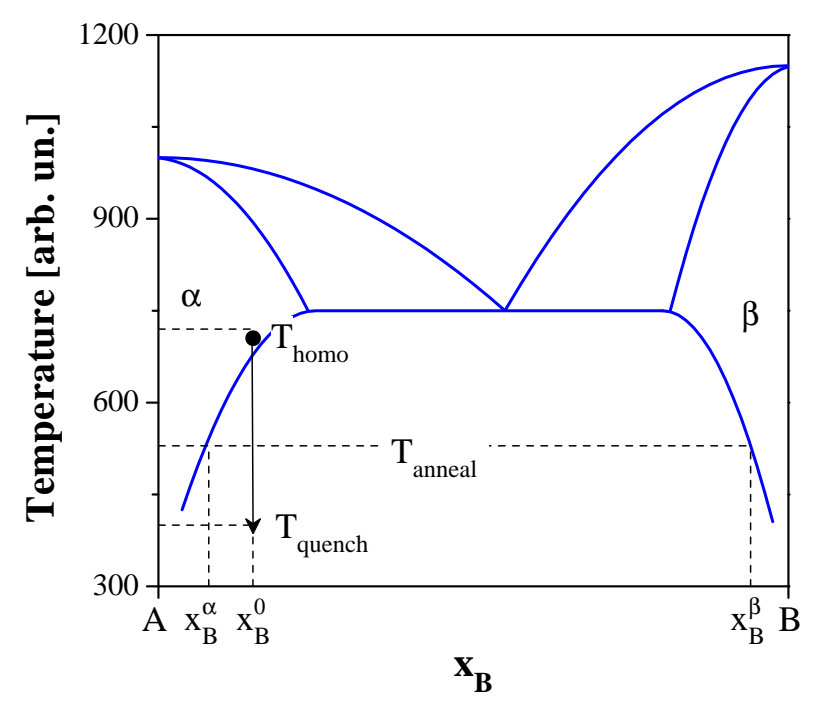

Figure 3.2. Scheme of a phase diagram in a $A-B$ binary system. The arrow shows the path of one of the possible solid state transformation or precipitation process in the case of an initial concentration of $B$ atoms equal to $x_{\mathrm{B}}^{0}$.

At this point one has particles of the $\beta$-phase (concentration $x_{\mathrm{B}}^{\beta}$, see figure 3.2 with radius $r>r^{*}$ which grow in a matrix ( $\alpha$-phase) progressively reducing the supersaturation

it results that

$$
r^{*} \propto 1 / \Delta T
$$

and therefore:

$$
W_{r^{*}} \propto 1 /(\Delta T)^{2}
$$


$S_{0}$

$$
S_{0}=\frac{x_{\mathrm{B}}^{0}-x_{\mathrm{B}}^{\alpha}}{x_{\mathrm{B}}^{\beta}-x_{\mathrm{B}}^{\alpha}}
$$

and small precipitates with $r<r^{*}$ which dissolve. The concentration in the matrix at the interface particle-matrix is very close to the equilibrium concentration $x_{\mathrm{B}}^{\alpha}$ while far from the nucleus it has still the original value $x_{\mathrm{B}}^{0}$. This results in a concentration gradient and consequently in a mass flow of atoms towards the growing particle. The growth of the particle is limited by the flux of atoms and hence by the diffusion coefficient as well as the geometry of the particle.

By solving the problem of the calculation of the concentration profile outside the particle, it can be then demonstrated that the rate with which the nuclei grow is expressed by the relation 133

$$
\frac{d r}{d t}=\frac{K_{\text {growth }}}{r}
$$

which gives after integration the evolution of the growth process in isothermal condition (considering $K_{\text {growth }}$ constant during the growth):

$$
r^{2}=r_{0}^{2}+K_{\text {growth }} \cdot\left(t-t_{0}\right)
$$

$K_{\text {growth }}$ is a function of thermodynamic and geometrical conditions which depend on the type of growth process taking place (for example: in supersaturation or supercooling conditions). In a supersaturated matrix the growing rate becomes a function of $S_{0}$ and of the diffusivity $D\left(K_{\text {growth }}=D S_{0}\right)$ and thus, in a real system, time or temperature dependent. An example of growing rate in such conditions is schematically shown in

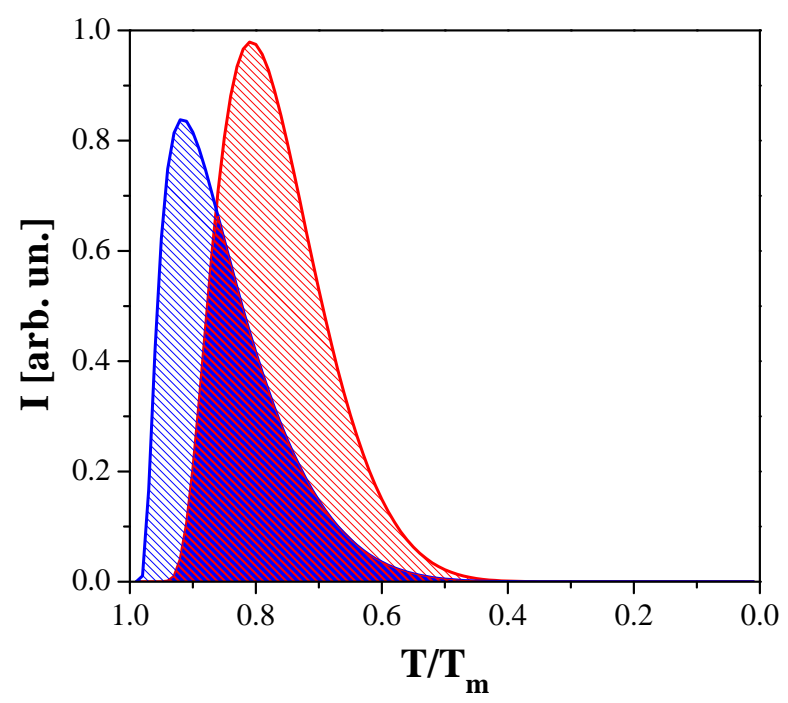

Figure 3.3. Schematic temperature dependence of the nucleation $(-)$ and growth $(-)$ rates $I$ 's below the melting temperature $T_{m}$.

figure 3.3 . The rate $I_{\text {growth }}$ increases rapidly and then decreases continuously towards 
zero when the supersaturation (or supercooling) becomes low. In a similar way, the excess energy that is released during the entire growing process in form of heat shows also a bell-shaped profile which reaches a maximum at the temperature where the growing rate is maximal. Thus the nucleation-and-growth (crystallization) can be followed by differential scanning calorimetry and studied by a simple chemical kinetic model (collision model) which permits also to justify the expression of the transformation rate $I$ as given by equation 3.9 .

\subsubsection{Reaction Kinetics in Differential Scanning Calorimetry}

In differential thermal analysis (DTA) or differential scanning calorimetry (DSC) the change in heat content or in thermal properties of a sample undergoing any reaction or transformation is indicated by a change of heat flow. If the transformation proceeds at a rate varying with temperature ${ }^{(5)}$ the position of the peak varies with the heating rate if other experimental conditions are maintained fixed. This evidence gives the possibility of studying the kinetics of the thermodynamic transformation taking place in the sample and to quantify it by calculating two important parameters: the activation energy and the frequency factor of the reaction.

Most reactions of the type solid $\rightarrow$ solid $(+$ gas $)$ can be described at constant temperature by the equation 91,92

$$
\left(\frac{\partial X}{\partial t}\right)=K(1-X)^{n}
$$

where $X$ is the fraction of material reacted, $K$ is the rate constant, and $n$ is the empirical order of reaction (in most cases, the exponent $n$ is unity or nearly unity and remains constant through the greater part of the reaction).

The rate $K$ is a function of the temperature $T$ and rapidly increases as the temperature rises. A simple explanation that accounts for the temperature dependence of the constant rate is provided by the collision model of chemical kinetics which assumes that atoms/molecules must collide to react. Increasing temperature increases molecular velocity and atoms collide more frequently and with more energy, leading to the consequent rise of the reaction rate. In addition to that, in 1888 the Swedish chemist Svante Arrhenius noted that, in order to react, colliding molecules must have a total kinetic energy equal to or greater than some minimum value which represents the energy necessary to force the reactant to move from its initial state through an unstable intermediate state to the final product (see figure 3.4). The minimum energy required to initiate a chemical reaction is called the activation energy $\left(\Delta E_{c}\right)$ and represents the energy barrier between the initial state and the highest energy along the transformation pathway.

${ }^{(5)}$ This is for example the case of thermal activated transformations characterized by activation energies. 


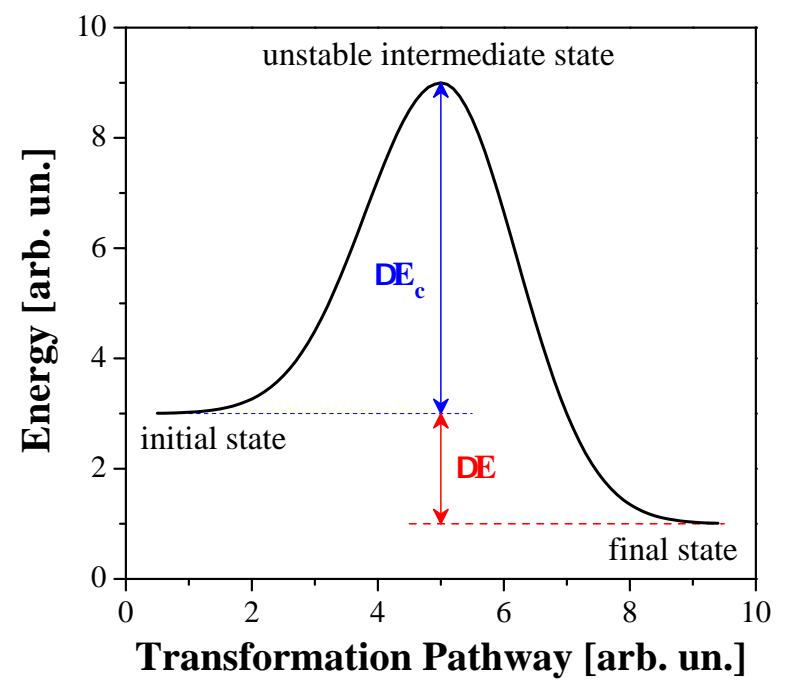

Figure 3.4. Energy profile for a generic transformation process. The atoms must surmount the activation-energy barrier $\Delta E_{c}$ before they can reach the final stable state. The energy change $\Delta E$ of the transformation has no effect on the rate but determines the sign (endothermic or exothermic) of the relative peak appearing at the transformation temperature in a DSC measurement.

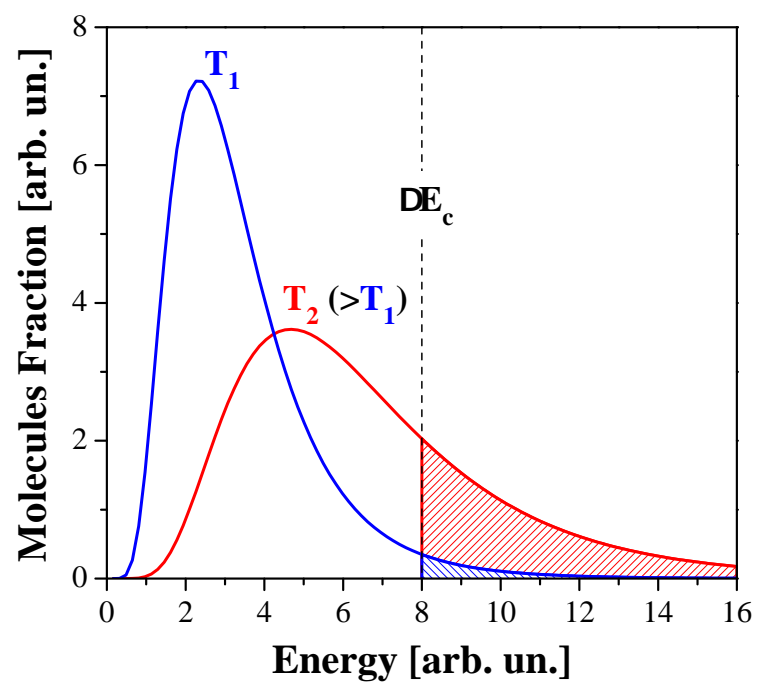

Figure 3.5. Distribution of kinetic energies in a sample of gas molecules at two different temperatures. At the higher temperature, a larger number of molecules has higher energy. Thus, a larger fraction at any one instant will have more than the minimum energy required for reaction. 
Taking into account the distribution of kinetic energies of a set of molecules at two different temperatures $T_{2}$ and $T_{1}$ and combining the collision model with the concept of activation energy, it becomes evident from figure 3.5 why at temperature $T_{2}\left(>T_{1}\right)$ a much greater fraction of the molecules has kinetic energy greater than $\Delta E_{c}$, and thus $K\left(T_{2}\right)>K\left(T_{1}\right)$.

On the basis of the previous considerations Arrhenius suggested the following relation for the magnitude of the reaction rate $K$ :

$$
K=K_{0} \cdot \exp \left(-\frac{\Delta E_{c}}{k_{B} T}\right)
$$

where $K_{0}$ and $\Delta E_{c}$ are constants which are properties of the material. The parameter $K_{0}$, often called the frequency factor, is a measure of the probability that a molecule having energy $\Delta E_{c}$ will participate in the reaction.

When the temperature changes with time, the variation of the transformed fraction becomes $(6)$ :

$$
\frac{d X}{d t}=\left(\frac{\partial X}{\partial t}\right)_{T}+\left(\frac{\partial X}{\partial T}\right)_{t} \cdot \frac{d T}{d t}=\left(\frac{\partial X}{\partial t}\right)_{T}
$$

and the total rate of reaction may then be expressed by the relation:

$$
\frac{d X}{d t}=K_{0}(1-X)^{n} \cdot \exp \left(-\frac{\Delta E_{c}}{k_{B} T}\right)
$$

independent on whether $T$ is constant or changes with time. As the transformation proceeds with increasing temperature, the transformation rate $d X / d t$ will rise to a maximum value, then return to zero as the reactant is exhausted. Considering a temperature rise at a constant rate $\beta$,

$$
T=T_{0}+\beta \cdot t
$$

where $T_{0}$ is the initial temperature, and by differentiation of equation 3.16, it results:

$$
\frac{d}{d t}\left(\frac{d X}{d t}\right)=\frac{d X}{d t}\left[\frac{\beta \Delta E_{c}}{k_{B} T^{2}}-K_{0} \cdot n(1-X)^{n-1} \cdot \exp \left(-\frac{\Delta E_{c}}{k_{B} T}\right)\right]
$$

The maximum transformation rate occurs at a temperature $T_{p}$ defined by setting $[d(d X / d t) / d t]_{T_{p}}=0:$

$$
\frac{\beta \Delta E_{c}}{k_{B} T_{p}^{2}}=K_{0} \cdot n(1-X)^{n-1} \cdot \exp \left(-\frac{\Delta E_{c}}{k_{B} T_{p}}\right)
$$

\footnotetext{
${ }^{(6)}$ The rate of change $(\partial X / \partial T)_{t}$ is obviously zero because considering the time as constant also fixes the number and position of the particles constituting the system and the only effect of an instantaneous change in temperature is in the velocity of thermal motion of the particles.
} 
The temperature $T_{p}$ is the sample temperature at which the peak differential thermal analysis deflection occurs $(7)$.

The product $n(1-X)^{n-1}$ in equation 3.19 is not only independent of $\beta$, but is very close to unity, and therefore it results:

$$
\frac{\beta \Delta E_{c}}{k_{B} T_{p}^{2}} \simeq K_{0} \cdot \exp \left(-\frac{\Delta E_{c}}{k_{B} T_{p}}\right)
$$

and

$$
\ln \left(\frac{\beta}{T_{p}^{2}}\right)=-\frac{\Delta E_{c}}{k_{B}} \cdot \frac{1}{T_{p}}+\ln \left(\frac{K_{0} k_{B}}{\Delta E_{c}}\right)
$$

regardless of reaction order. The plot $\ln \left(\beta / T_{p}^{2}\right)$ versus $1 / T_{p}$ derived from differential thermal analysis patterns at a number of heating rates makes possible the determination of the activation energy $\Delta E_{c}$ and of the frequency factor $K_{0}$ for a simple decomposition reaction by fitting the resulting linear curve.

${ }^{(7)}$ The temperature distribution in the DTA or DSC specimen holders obeys the general heat flow equation:

$$
\frac{\partial T}{\partial t}-\frac{k}{\rho c} \nabla^{2} T=\frac{1}{\rho c} \frac{d q}{d t}
$$

where $T$ is the temperature, $t$ the time, $k$ the thermal conductivity, $\rho$ the density, $c$ the specific heat, and $d q / d t$ the rate of heat generation due to a chemical reaction per unit volume of sample. No heat effects occur in the reference sample, so the temperature distribution in the reference is given by:

$$
\frac{\partial T}{\partial t}=\frac{k}{\rho c} \nabla^{2} T
$$

If the reference sample is assumed to be a cylinder of radius $r$ and of infinite length, with the temperature of the outside given by $T=T_{0}+\beta t$, the temperature $T_{\text {reference }}$ at the center of the sample is:

$$
T_{\text {reference }}=T_{0}+\beta t-\frac{\beta \rho c r^{2}}{4 k}
$$

as obtainable by integration of equation 3.21 . In a similar way the solution expressing the temperature at the center of the active sample will be of the form:

$$
T_{\text {sample }}=T_{0}^{\prime}+\beta t-f\left(\frac{d q}{d t}\right)
$$

where $f\left(\frac{d q}{d t}\right)$ is a function of the rate of the reaction. The differential temperature is the difference in temperature of the centers of the two samples and it is given by:

$$
\Delta T \simeq f\left(\frac{d q}{d t}\right)-\frac{\beta \rho c r^{2}}{4 k}
$$

and

$$
\frac{d(\Delta T)}{d t}=f^{\prime}\left(\frac{d q}{d t}\right) \frac{d^{2} q}{d t^{2}}
$$

When $\Delta T$ is a maximum, $d(\Delta T) / d t$ is zero and due to equation $3.25 d^{2} q / d t^{2}=0$ which states that the peak in a DTA or DSC analysis at constant heating rate $\beta$ occurs when the reaction rate $d q / d t$ (or $d X / d t$, due to the proportionality between the heat generation rate and the reacted fraction rate) is a maximum. 
An example of Kissinger's plot as derived from the analysis of the primary crystallization of the amorphous phase in $\mathrm{Nd}_{60} \mathrm{Fe}_{30} \mathrm{Al}_{10}$ bulk samples is reported in figure 3.6. together with the relative temperature variation of the transformation rate $K_{p}$.
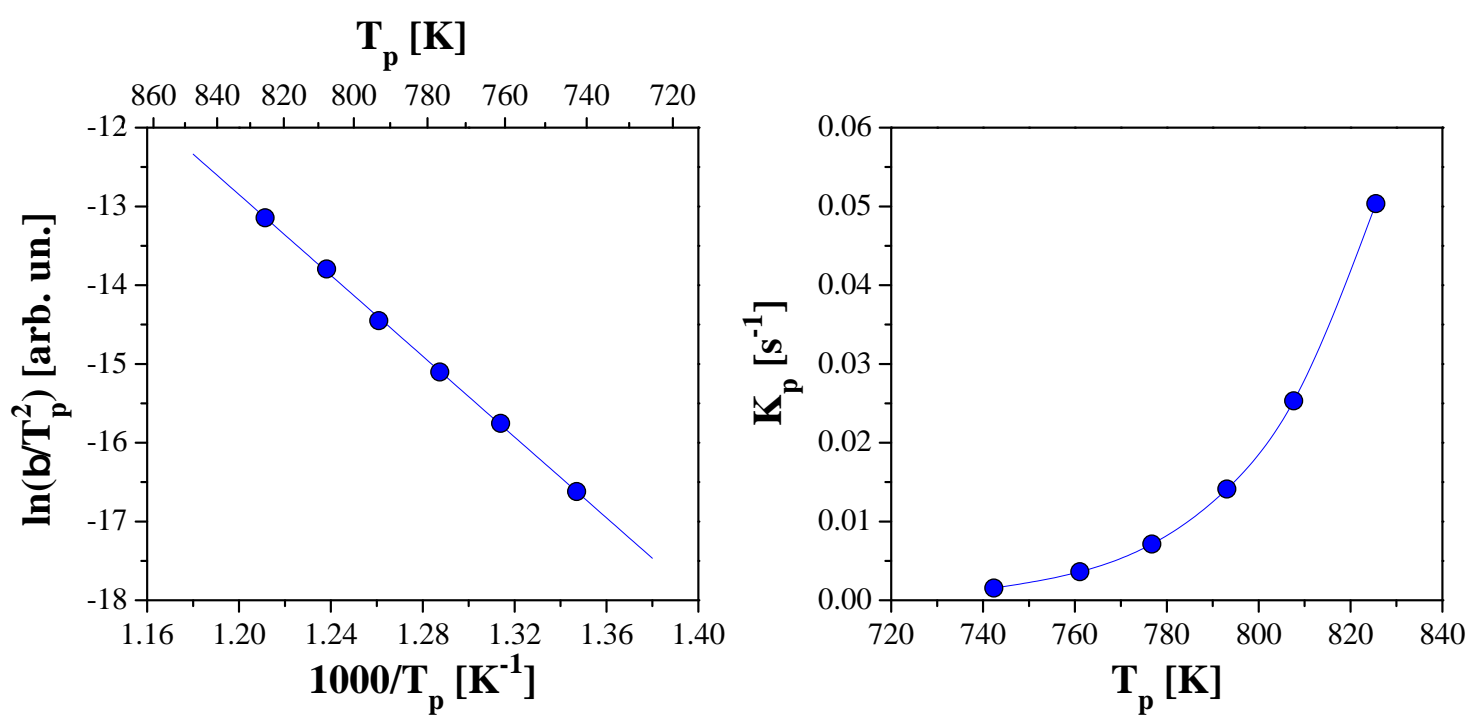

Figure 3.6. (Left) Kissinger's plot of $\mathrm{Nd}_{60} \mathrm{Fe}_{30} \mathrm{Al}_{10}$ bulk (•) sample and the relative linear fit. (Right) Temperature dependence of the transformation rate $K_{p}$ for the $\mathrm{Nd}_{60} \mathrm{Fe}_{30} \mathrm{Al}_{10}$ bulk sample.

\subsubsection{Continuous-Heating-Transformation (CHT) Diagram}

The Kissinger's analysis has a significant extension which goes well beyond the calculation of the activation energy and of the frequency factor of the material. In fact, knowing the values of $\Delta E_{c}$ and $K_{0}$ gives the possibility through equation 3.27 to calculate for any crystallization temperature $T_{p}$ the appropriate rate $\beta$ which must be used in a DSC scan to measure the transformation peak at $T=T_{p}$ :

$$
\beta=T_{p}^{2} \cdot \exp \left(-\frac{\Delta E_{c}}{k_{B}} \cdot \frac{1}{T_{p}}\right) \cdot\left(\frac{K_{0} k_{B}}{\Delta E_{c}}\right)
$$

and, inverting equation 3.17 , it results:

$$
t_{p}=\left(T_{p}-T_{0}\right) / \beta
$$

which permits to calculate the time $t_{p}$ necessary to fully complete the reaction undergoing in the sample. The set of points $\left\{t_{p}, T_{p}\right\}$ determines a continuous heating transformation (CHT) diagram which allows to predict the transformation temperature $T_{p}$ for any heating rate without running DSC measurements. Such temperature is the intersection at which the heating curve $T=T_{0}+\beta \cdot t$ crosses the transformation plot $\left\{t_{p}, T_{p}\right\}$.

An example of such transformation diagram is reported in figure 3.7 as derived from the above-described analysis for $\mathrm{Nd}_{60} \mathrm{Fe}_{30} \mathrm{Al}_{10}$ samples. 


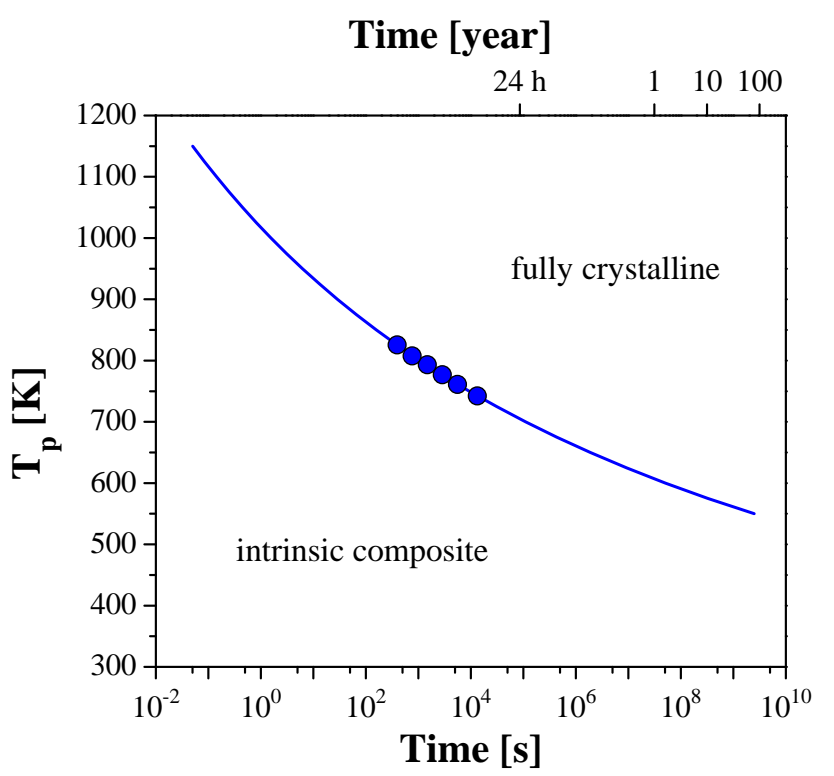

Figure 3.7. Pseudo time-temperature-transformation diagrams of $\mathrm{Nd}_{60} \mathrm{Fe}_{30} \mathrm{Al}_{10}$ bulk samples.

The CHT diagram describes the course of a transformation in the same way as the socalled time-temperature-transformation (TTT) diagram follows the crystallization process competing with glass formation during cooling (and undercooling) from the melt of a glass-forming material ${ }^{(8)}$. Due to the analogy between CHT and TTT curves, we will call the continuous heating transformation curve also pseudo time-temperaturetransformation diagram and it will used in chapter 6 to compare the thermal stability of different samples against crystallization during thermal treatments and quasiinstantaneous annealing processes.

\subsection{Coarsening}

As seen above, a system which undergoes a nucleation-and-growth process results in a dispersion of $\beta$-phase particles embedded in an $\alpha$-matrix which may be not in equilibrium due to the considerable quantity of energy associated with the $\alpha-\beta$ interface. Usually, at this point (low level of supersaturation or supercooling) a second process starts to play an important role: coarsening. Coarsening, also called Ostwald ripening or competitive growth, defines the event in which large particles grow at the expense of small ones. Since the system tries to minimize the energy and the nucleation-and-growth cannot proceed due to high undercooling or low supersaturation, the amount of interfacial energy is

\footnotetext{
${ }^{(8)}$ In the case of a glass-forming material the term TTT diagram is conventionally used for the timetemperature-transformation curve of a sample which is cooled from the liquid phase down to any temperature $T^{\prime}$ and then isothermally annealed for a time $t^{\prime}$ until the transformation amorphous $\rightarrow$ crystalline state takes place. The set of points $\left\{t^{\prime}, T^{\prime}\right\}$ is properly a TTT curve [68, 105].
} 
reduced by decreasing the number of small particles which have more interfacial area than one large particle with the same volume. Hence a dispersion of particles of a certain phase having a certain distribution of radii which are embedded in a host matrix, coarsens (or ripens) and an increase in the spatial separation between neighboring particles results, together with a decrease of their number, and with a nearly constant volume fraction $\left(V_{\text {particle }} / V_{\text {system }}\right)$.

As seen in the previous section, the growth equation 3.11 establishes the rate at which a nucleus of radius $r$ grows independently from the particles dispersion and size distribution. In the case of coarsening, a new situation must be taken into account: the solute $\left(x_{B}\right)$ concentration in the matrix adjacent to a particle will increase as the radius of curvature of the particle decreases (Thomson-Freundlich effect). Thus the solubility in the matrix in contact with a $\beta$ particle increases as the radius of the particle decreases and, hence, a concentration gradient (and consequently a gradient of the chemical potential) develops in the matrix determining a driving force for the solute which diffuses in the direction of the largest particles and away from the smallest particles (see figure 3.8). The competitive

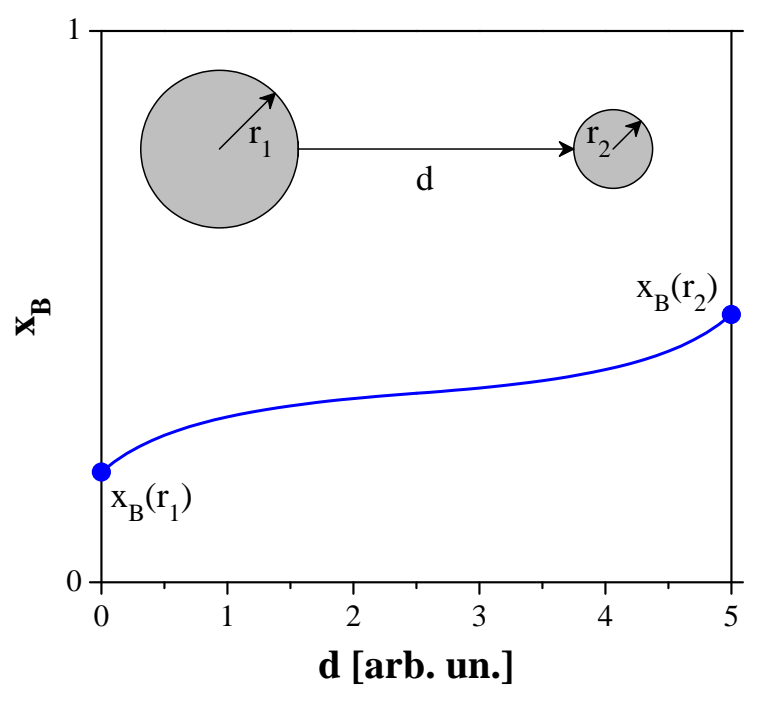

Figure 3.8. Concentration profile between two precipitates with different radii $\left(r_{1}>r_{2}\right)$.

growth of large particles and dissolution of small ones continues until the interfacial energy is minimized (and the gradient of the chemical potential becomes zero).

A general form of growing rate for Ostwald ripening under diffusion transport of heat or mass is expressed by the relation:

$$
\frac{d r}{d t}=\frac{K_{\text {coarsening }}}{r^{2}}\left(\frac{r}{r_{c}}-1\right)
$$

where $K_{\text {coarsening }}$ is a rate constant which includes, depending on the type of ripening process, all the physical parameters describing the growth. The radius $r_{c}$ is called coarsening critical radius and, as clear from equation 3.30, says that particles having that radius have 
exactly a zero growth rate. On the contrary, particles with $r<r_{c}$ shrink and dissolve while particles with $r>r_{c}$ grow. This singular behavior can be probably better understood considering a hyper simplified picture of a single phase system containing particles which have nucleated upon undercooling. When a huge number of spheres with different radii are embedded in the matrix, the matrix temperature is not in equilibrium with all the particles each of which has a slightly different melting temperature depending on its radius (Gibbs-Thomson effect). Thus, the temperature gradient existing in the matrix leads to melting of the small spheres which have $T_{m}^{\text {particle }}<T_{\text {matrix }}$ and to growth of the big ones which have on the contrary $T_{m}^{\text {particle }}>T_{\text {matrix }}$. In such a way, however, the equilibrium temperature $T_{m}^{\text {matrix }}$ and the critical radius $r_{c}$ are constant parameters but vary with time making the analytical integration of equation 3.30 impossible.

Nevertheless several approximated solutions have been successfully proposed to describe the coarsening process. Among them, the works of Lifshitz and Slyozov 108, Wagner [166], and Marqusee and Ross 116, 133 are nowadays widely accepted as mathematical solutions of equation 3.30. Wagner simplified the analysis by assuming that the volume fraction of the particles is a conserved quantity. In this way he calculated that the average radius $\langle r\rangle$ of the particle dispersion, in the case of diffusive coarsening, can be expressed as

$$
\langle r\rangle=r_{c}(t)
$$

from which the evolution of mean radius becomes:

$$
\langle r\rangle^{3}=\frac{12}{27} K_{\text {coarsening }} \cdot t
$$

and the number density of particles:

$$
n_{V}(t) \propto \frac{1}{t}
$$

Marqusee and Ross [116] integrated the work of Wagner proposing in 1983 a more complete solution of competitive growth. They suggested that the conservation of mass or enthalpy is a more general hypothesis than the simple conservation of the volume fraction and they obtained a relation for the late stage of the coarsening when the system reaches a steady-state which can be summarized by the time-dependent expressions:

$$
\begin{aligned}
\langle r\rangle & =\frac{8}{9} r_{c}(t) \\
\langle r\rangle^{3} & =K_{\text {coarsening }}^{\prime} \cdot t \\
n_{V}(t) & \propto \frac{1}{t}
\end{aligned}
$$

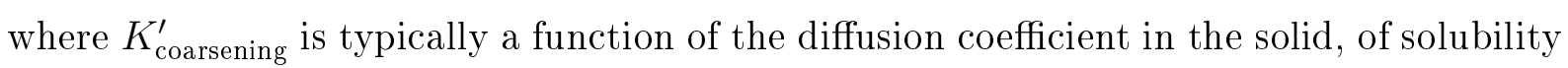
of the solute in the matrix, and of the interfacial energy.

Even if the results of Marqusee and Wagner present several similarities, many differences would emerge from a deeper description of the two solutions. One of the most 
important one, which affects the analysis presented in the next section, concerns the volume fraction of the particles in the matrix. While Wagner considered it as a constant, Marqusee showed that the volume of the particles changes with time and, only after long annealing times, it becomes asymptotically time-independent ${ }^{(9)}$,

The growth laws presented here indicating the cube root of time dependence of the radius and the reduction of number of particles are generally observed and confirmed experimentally. However, as in the case of nucleation-and-growth, the separation of forming a two phase alloy from a single phase into the steps:

(i) nucleation

(ii) growth of the nuclei or particles

(iii) coarsening (Ostwald ripening)

is artificial and the tendency is to unify the three processes into a unique process and a unique theory. From an experimental point of view, however, the necessity to clearly identify the kind of process playing the primary role in a transformation process justifies such schematic distinction as it is done in the next section which deals with the experimental characterization of a system undergoing coarsening.

\subsubsection{Experimental Investigation of Coarsening in Composite Materials}

For the experimental investigation of coarsening in the $\mathrm{Nd}_{60} \mathrm{Fe}_{30} \mathrm{Al}_{10}$ intrinsic composite a set of assumptions and approximations must be considered. We will assume a general coarsening process in which larger grains in a population grow on average at the expense of the smaller ones under the driving force determined by the decrease in interfacial free energy of the whole system. If the interfacial tension $\gamma$ and the mobility $m$ of a grain boundary are assumed constant, the increase in the average radius $r$ of the grains can be written as:

$$
\frac{d r}{d t}=\frac{c m \lambda^{n} \gamma}{r^{n-1}}
$$

where $c$ is a numerical factor, $\lambda$ is the interatomic distance, and $n$ is the growth exponent. As previously demonstrated, theoretically $n=2$ for nucleation-and-growth, 3 for Ostwald ripening, while values of $n$ between 1.5 and 4 (see [34] and references therein) have been observed experimentally for polycrystalline alloys, between 3 and 4 for fractal viscous

\footnotetext{
${ }^{(9)}$ This result is a direct consequence of the conservation of mass/energy which is a more physical principle than the conservation of the volume fraction. On the case of coarsening, when the supersaturation or the temperature in a dispersion are very close to their equilibrium values, the differences are small and the mass/enthalpy conservation and the volume conservation are merely identical.
} 
fingering patterns [142], and greater than 4 for computer simulations of an intrinsic 2-D composite 60 (10).

Standard transition state rate theory gives the mobility in terms of microscopic quantities as:

$$
m=\frac{\nu \lambda^{2}}{k_{B} T}
$$

where $\nu$ is the net jump frequency across the boundary which is usually assumed to be a single activated process with the following temperature dependence:

$$
\nu=\nu_{0} \cdot \exp \left(-\frac{\Delta E_{c}}{k_{B} T}\right)
$$

The growth rate expressed in equation 3.37 can be integrated to give the time evolution of the grain size as

$$
r^{n}(t)=r^{n}(0)+\int_{0}^{t} k(T) d t
$$

where:

$$
k(T)=n c m \lambda^{n} \gamma
$$

is called the reaction rate constant. For convenience, the temperature dependence of $k(T)$ can be rewritten a: (11),

$$
k(T)=\frac{k_{0}}{T} \cdot \exp \left(-\frac{\Delta E_{c}}{k_{B} T}\right)
$$

Thus in an isothermal experiment, the evolution of the grain size can be expressed in analogy with equation 3.12 and equation 3.32 by the relation:

$$
r^{n}(t)=r^{n}(0)+k(T) \cdot t
$$

while in a scanning experiment at constant heating rate $\beta$, the grain size variation becomes:

$$
r^{n}(t)=r^{n}(0)+\int_{0}^{T} \frac{k(T)}{\beta} d T
$$

which can be rewritten for clarity as:

$$
r(t)=\left(r^{n}(0)+\int_{0}^{T} \frac{k_{0}}{\beta T} \cdot \exp \left(-\frac{\Delta E_{c}}{k_{B} T}\right) d T\right)^{1 / n}
$$

\footnotetext{
${ }^{(10)}$ Time/temperature dependence of the growth exponent $n$ and transition from one regime to an other were also reported.

${ }^{(11)}$ The rate constant $k(T)$ is sometimes also assumed to have a simple Arrhenius-type temperature dependence:

$$
k^{\prime}(T)=k_{0}^{\prime} \cdot \exp \left(-\frac{\Delta E_{c}^{\prime}}{k_{B} T}\right)
$$

which derives from an incorrect treatment of the frequency factor in the expression of the mobility $m$. Although equation 3.42 differs significantly from equation 3.43 , it can be shown that the difference between the activation energies $\Delta E_{c}$ and $\Delta E_{c}^{\prime}$ is usually negligible.
} 
The evolution of the grain size is accompanied by a heat release as consequence of the minimization of the interfacial energy which can be detected in a calorimetric experiment if favorable experimental conditions exist. The height of the calorimetric signal can be estimated considering the temperature derivative of the interfacial enthalpy. If this is assumed equal to

$$
H=\frac{g \gamma_{H} V}{r(T)}
$$

where $\gamma_{H}$ is the enthalpic part of the interface tension, $V$ is the volume, $r$ the average grain radius of the growing phase, and $g$ is a numerical factor with a value close to unity and which depends on the shape and size distribution of the grains. The calorimetric signal (heat flow) is the rate $d H / d T$ at which the enthalpy of the sample changes (a negative quantity for exothermal processes). Now, assuming that the volume $V$ remains nearly constant, the evolution of the interfacial enthalpy can be rewritten as:

$$
H(T)=\frac{H_{0} r_{0}}{r(T)}
$$

where $H_{0}$ and $r_{0}$ are the initial enthalpy and the initial grain radius, respectively. Hence the heat rate $d H / d T$ can be expressed by the relation

$$
\frac{d H}{d T}=-\frac{H_{0} r_{0}}{r(T)} \frac{d r}{d T}=-\left(\frac{H_{0} r_{0}}{n}\right) \frac{k(T)}{r^{n+1}}
$$

which express the temperature evolution of the heat release associated with the coarsening process. This energy can be measured independently by DSC and compared to the value arising from the previous theoretical model and given by equation 3.49 .

For comparison, the enthalpy release rate in an isothermal nucleation-and-growth process is expressed instead by the relation [34

$$
\frac{d H}{d t}=-\Delta H(1-x) n b t^{n-1}
$$

where $\Delta H$ represents the total transformation enthalpy of the sample and $x$ is the fraction of material transformed after a time $t$ which is expressed according to the Johnson-MehlAvrami analysis 75 as

$$
x=1-\exp \left(-b t^{n}\right)
$$

where $b$ is a constant dependent on the nucleation frequency and $n$ the growth exponent ( $2<n<4$ depending on the nucleation mechanism). At this point it must be noted that the enthalpy rates arising from a coarsening process (equation 3.49) and from nucleationand-growth (expressed by applying equation 3.50 to a series of short isothermals anneals in a linear heating calorimetric simulation) present the same behavior characterized by a bell-shaped curve. Although the mechanisms driving the two processes are of completely different nature, their calorimetric signals in non-isothermal conditions are similar and, therefore, their distinction in a DSC scan is commonly a challenging task [34, 55]. 


\section{Chapter 4}

\section{Magnetism in Amorphous and Composite Materials}

Intrinsic composite are a fascinating class of materials not only for their structural properties and the possibility to tailor their microstructure (see section 3) but also for their potential as new magnetic materials. In fact, the presence of two (or more) ordered/disordered interacting phases which have different chemical compositions and magnetic properties is an important prerequisite for the appearance of new magnetic effects which cannot be observed in a single-phase uniform system.

To understand the mechanisms and the magnetic features which characterize such effects, the basic requirements for magnetism in solid materials must be considered and the consequences which structural disorder may have on them must be taken into account. Section 4.1 is dedicated to those issues. Section 4.2 will then show which types of magnetic collective order can arise in disordered magnetic materials and it will introduce to the random anisotropy model which is a wide accepted theoretical model for the description of those systems (section 4.3. Section 4.4 introduces briefly to the principal magnetization mechanisms responsible for the hysteretic behavior of magnetic materials while section 4.5 will describe the theoretical model of Gaunt which accounts for the domain wall pinning processes. In particular, section 4.5.1.2 will face the problem of how structural features may act as pinning centers for the domain wall motion in non-homogeneous samples, and determines therefore a link between magnetic and structural features.

\subsection{Effect of Disorder on Magnetic Properties}

The basic requirements for magnetic order in a solid are the existence of atoms with a magnetic moment and an interaction which couples them together (exchange coupling). If the solid is a perfect crystal of a pure element, it can be assumed that each atom has the same magnetic moment and each atom-pair the same interaction strength. Therefore 
it must be presumed that structural and chemical disorder in heterogeneous materials (e.g., alloys, amorphous systems, or intrinsic composites) introduce a distribution both in the magnetic moments and in the exchange coupling, and determine a magnetic order consisting of site-dependent interactions. Such local variations of the magnetic, structural and chemical properties may also affect the electrostatic fields in the solid, thereby, determining site-dependent orientation of the magnetic moments and, consequently, magnetic anisotropy which varies locally in direction and magnitude.

Hence, the microstructure and its degree of order may strongly affect magnetism in amorphous and composite materials through various effects which they have on magnetic moments, exchange coupling, and magnetic anisotropy. The next sections are dedicated to the description of those effects and, particularly, to their relevance on the magnetic behavior of rare-earth-transition metal (RE-TM) systems.

\subsubsection{Effects of Disorder on Magnetic Moments}

A magnetic moment exists on a free atom whenever there are unpaired electrons. In a solid, however, these electrons participate to the formation of covalent bonds or electronic bands and loose therefore their strong paramagnetism. The only exceptions are the insulating and semiconducting compounds of the $3 \mathrm{~d}$ series and almost all $4 \mathrm{f}$ materials, whose unpaired electrons are localized in an inner shell and cannot therefore fully participate in the bonding, and some of the metals of the $3 \mathrm{~d}$ series [126].

The theory of the localized magnetism of non-interacting ions expresses the Hamiltonian as

$$
\mathscr{H}=\mathscr{H}^{C}+\mathscr{H}^{O S}+\mathscr{H}^{E F}
$$

where $\mathscr{H}^{C}$ is the Coulomb interaction among $\mathrm{f}$ (or d) electrons, $\mathscr{H}^{O S}$ is the spin-orbit interaction, and $\mathscr{H}^{E F}$ the interaction between electrostatic field ("crystal field") and localized electrons. The strength of each term varies strongly between the RE elements, where $\mathscr{H}^{O S}$ predominates, and $3 \mathrm{~d}$ TM for which $\mathscr{H}^{E F} \gg \mathscr{H}^{O S}$ (thereby "quenching" their orbital angular momentum). Therefore the relative magnitude of $\mathscr{H}^{C}, \mathscr{H}^{O S}$, and $\mathscr{H}^{E F}$ determines the magnetic moment of each element and local perturbation (structural disorder) and the presence of ligands elements (chemical disorder) reduce and broaden it from the free-ion value.

Magnetism in $3 \mathrm{~d}$ metals (Fe, Co, Ni), instead, must be discussed in terms of their electronic band structures since the magnetic moments of these metals and of their alloys are largely due to spin polarization of the d-electrons which are delocalized because of the overlap of the d-electrons wavefunctions of the neighboring sites and strong s-d hybridization. In band magnetism (itinerant or delocalized magnetism) the necessary condition for a stable ferromagnetism is the Stoner criterion 149

$$
J \cdot n\left(E_{f}\right)>1
$$


where $J$ is the electrons exchange constant and $n\left(E_{f}\right)$ is the electronic density of states (DOS) at the Fermi energy. The product $J \cdot n\left(E_{f}\right)$ for the 3 d metals is shown in figure 4.1 (bottom) 82 . The appearance of magnetism in those elements is near the limit

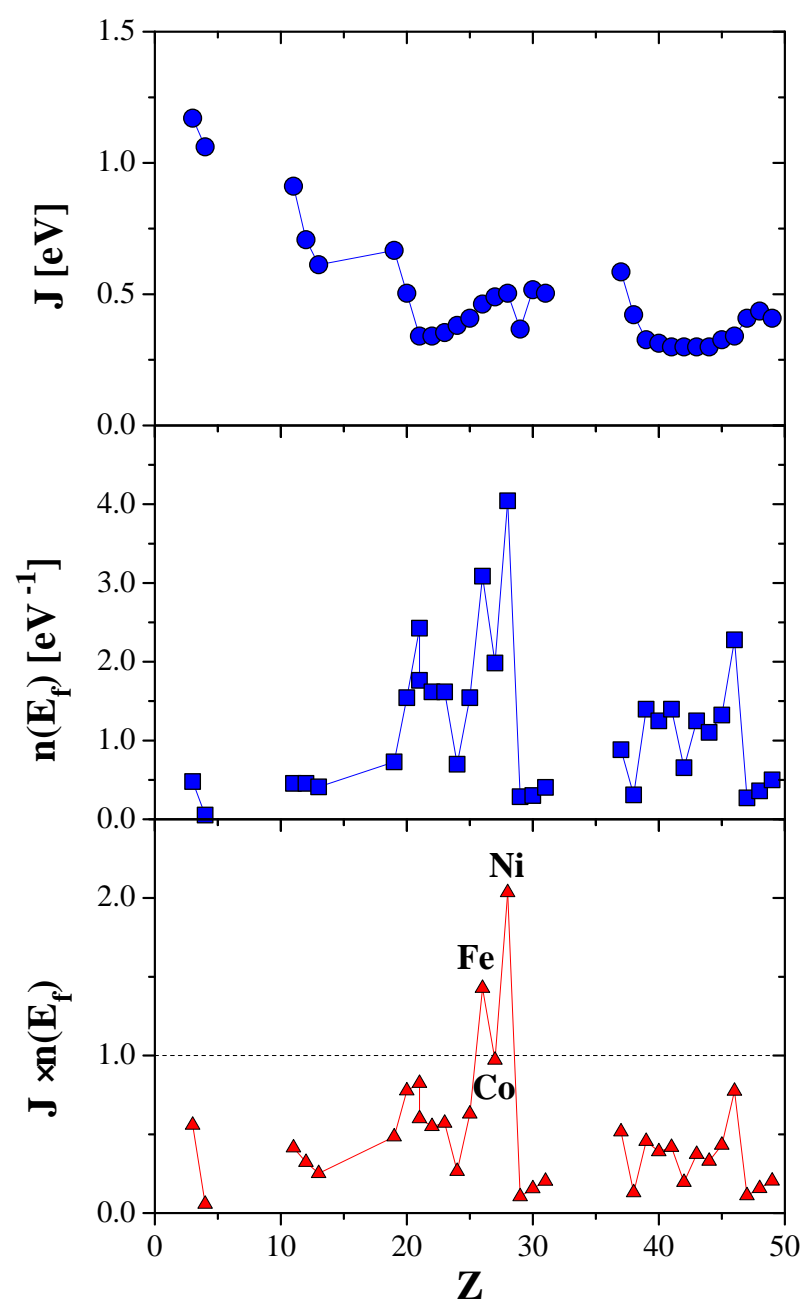

Figure 4.1. Calculated variation of the exchange constant $J$, density of states $n(E)_{f}$, and their product $J \cdot n(E)_{f}$ with the atomic number $Z$ for elements of the 3 d transition series. The dashed line $J \cdot n(E)_{f}=1$ defines the limit delineated by the Stoner criterion 82 .

of the Stoner criterion and their moments are all quite sensitive to changes on the overlap integrals from which the density of states at the Fermi level depends. Variations of the nearest-neighbor distances and of the nature of the surrounding conduction electrons broaden the local density of states in such a way that the magnetic moment may be reduced or even destroyed.

\subsubsection{Effects of Disorder on Exchange Interactions}

In the case of two localized electronic spins, they are aligned parallel or anti-parallel (Pauli principle) by exchange interaction, as phenomenologically described by the isotropic 
Heisenberg operator

$$
\mathscr{H}_{i j}=-2 J_{i j} \boldsymbol{S}_{i} \cdot \boldsymbol{S}_{j}
$$

where $g \mu_{B} S$ is the magnetic moment ( $g=2$ is the Landé factor for electrons) and $J_{i j}$ is the exchange constant between spins at site $i$ and site $j$.

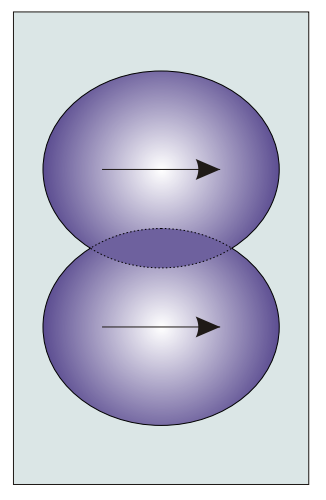

Direct Exchange

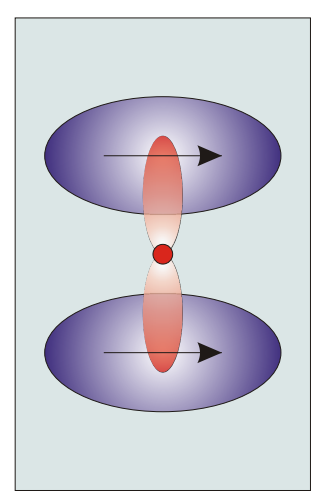

Super-Exchange

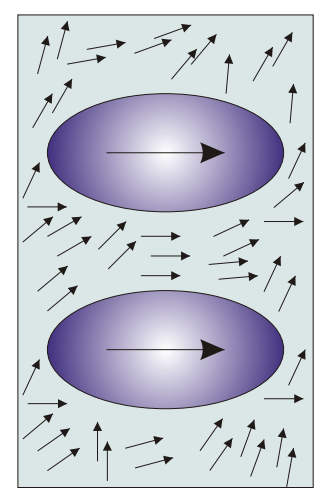

Indirect Exchange (RKKY Interaction)

Figure 4.2. Different exchange mechanisms responsible for collective magnetic order in magnetic materials.

Analogously, the appearance in a solid of magnetically ordered structure (e.g., ferromagnetism, antiferromagnetism, etc.) requires an interaction which couples the magnetic moments together the nature and the properties of such interaction are however more complex than those arising from equation 4.3 for two electronic spins. In fact, the origin of exchange coupling lies in the electrostatic interaction between electrons with different spins on different sites and, therefore, depends strongly on the magnetic and electronic properties of the local surroundings. As schematically illustrated in figure 4.2, various exchange mechanisms of different nature exist: direct exchange, superexchange via ligands, indirect exchange via conduction electrons (RKKY interaction) [13, all of them depending sensitively on the distance between the interacting electrons (see figure 4.3) or on the nature of the nearest-neighbor atoms (see figure 4.4.)

Exchange couplings are, however, not the only interactions which couple two magnetic moments, since also the classical dipolar interaction

$$
\mathscr{H}_{i j}^{D i p}=\frac{\boldsymbol{\mu}_{i} \cdot \boldsymbol{\mu}_{j}}{r_{i j}^{3}}-\frac{3\left(\boldsymbol{\mu}_{i} \cdot \boldsymbol{r}_{i j}\right)\left(\boldsymbol{\mu}_{j} \cdot \boldsymbol{r}_{i j}\right)}{r_{i j}^{5}}
$$

tends to align two moments $\boldsymbol{\mu}_{i}$ and $\boldsymbol{\mu}_{j}$ parallel along the line joining their sites $\left(r_{i j}\right)$. Like the RKKY interaction, the dipolar coupling varies as $1 / r_{i j}^{3}$ but it is much weaker than exchange and quite anisotropic due to the dependence from the orientation of the moments relative to $\boldsymbol{r}_{i j}$. Distributions of orientations and distances of the spins control therefore the local strength of the dipolar interaction and, hence, may affect the macroscopic domain 

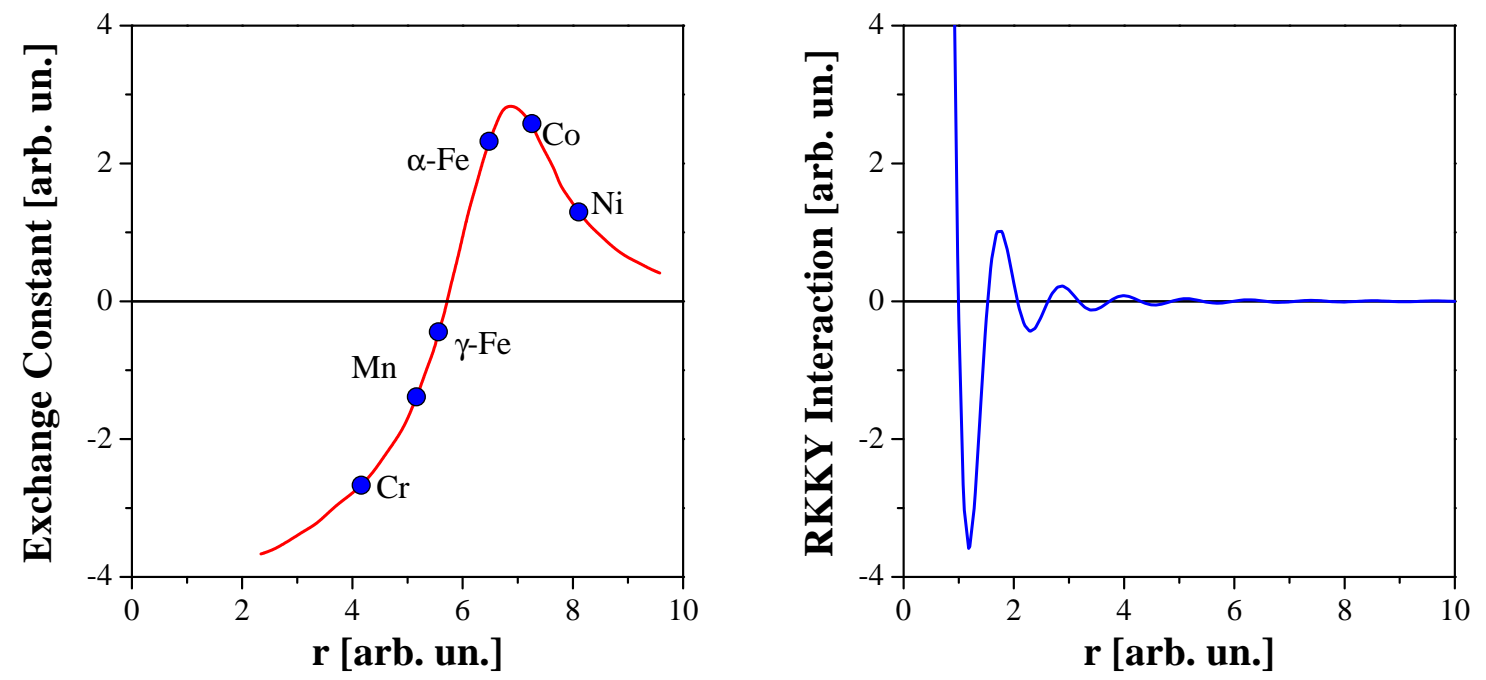

Figure 4.3. (Left) Schematic variation of exchange constant as a function of distance between $3 \mathrm{~d}$ shells in the $3 \mathrm{~d}$ transition metals. (Right) Dependence of the indirect exchange constant on the distance between two atoms coupling via the RKKY interaction.

structure which appears in the magnetically ordered states of crystalline and amorphous ferromagnets as consequences of demagnetizing effects that lower the overall energy of the system.

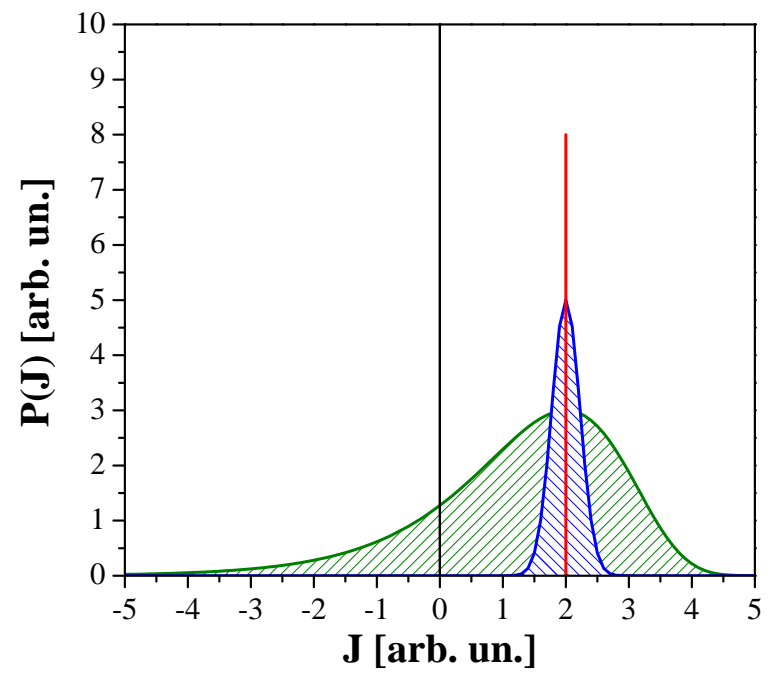

Figure 4.4. Schematic probability distributions of the direct exchange constant $J$ in a crystal $(-)$, and in amorphous solids with increasing amounts of disorder $(-,-)$.

\subsubsection{Effects of Disorder on Magnetic Anisotropy}

After exchange, the next most significant factor determining the properties of magnetic materials is single-ion anisotropy. Each atom, or ion, in a solid experiences an electrostatic 
field created by the charges of all the other ions, plus a contribution from any conduction electron. This field is known as the crystal field or ligand field in crystals or molecules, but in non-crystalline solids, it is commonly called electrostatic field. The effect of the electrostatic field on the magnetic moment of an atom is to confine it by spin-orbit coupling to certain preferred directions along which the energy of the system results to be minimized. The single-ion anisotropy at each atomic site in a perfect crystal has therefore the point symmetry of the site itself and the magnetization is for this reason preferable oriented along certain crystallographic orientations (magnetic easy axis and, consequently, hard axis). The presence of easy/hard axis can be understood if the strength of the magnetocrystalline anisotropy is taken into account in the computation of the total energy of the system (Stoner-Wohlfahrt model [12]). Commonly the magnetocrystalline energy term is expressed as

$$
E^{A n}=-K_{1} \cdot \cos (\theta)-K_{2} \cdot \cos ^{2}(\theta)+\ldots
$$

where $K_{i}$ is the anisotropy constant of order $i$ and $\theta$ is the angle between the magnetic moment $\boldsymbol{m}$ and the anisotropy easy axis.

Magnetocrystalline is of course not the only form of anisotropy. In fact magnetoelastic (magnetoelastic anisotropy), demagnetization (shape anisotropy) and interfaces/surfaces (interface anisotropy) effects are responsible too for the appearance of preferred orientations of the magnetic order in a sample under no external magnetic field.

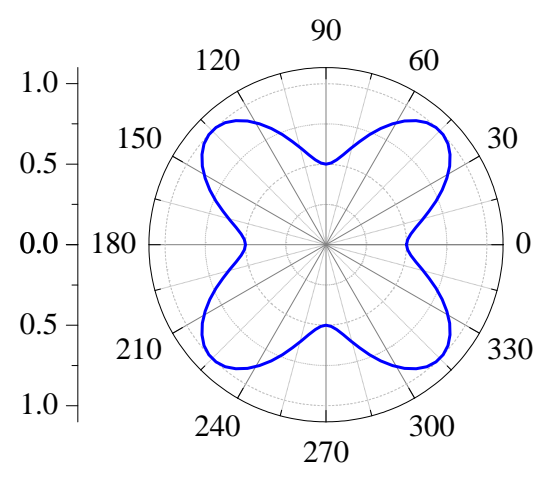

(a)

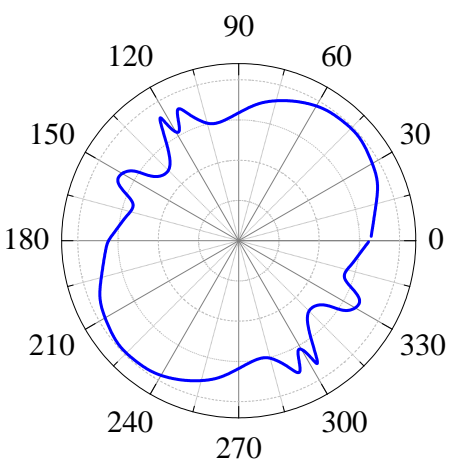

(b)

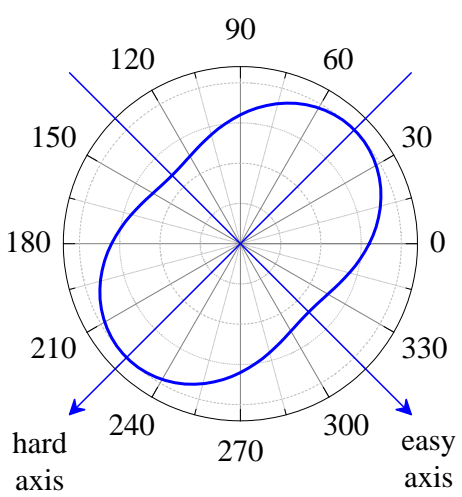

(c)

Figure 4.5. Two-dimensional anisotropy energy contour in a crystal (a), in an amorphous material with uniaxial anisotropy (b), and in the random axial anisotropy model of Harris, Pliske and Zuckermann (c) 72$]$.

In disordered systems, the values of the anisotropy constants $K_{i}$ and, hence, the strength of the magnetic anisotropy may vary strongly from site to site, depending on the local structural and chemical order, and the expression of the anisotropy energy may become a complex function due to the lack of symmetry and uniform properties.

A simplification may however arise for those amorphous systems for which the electrostatic interaction can be imagined to give a three dimensional energy contour with no 
particular symmetry. The projection of such energy contour on a plane is schematically shown for a perfect crystal (a) and for an amorphous material (b) in figure 4.5. If we assume that the interaction between two neighbor atoms (pair correlation) is not randomly distributed in all orientations but mostly close to one direction (see figure 4.6), one axis of the energy contour results to be lower in energy than all the others and the system shows anisotropic behavior with local easy axis that varies from site to site, leading to random axial anisotropy. This assumption is at the basis of the theoretical description

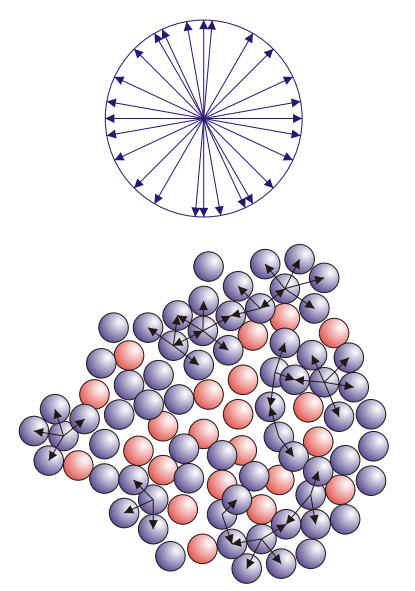

(a)

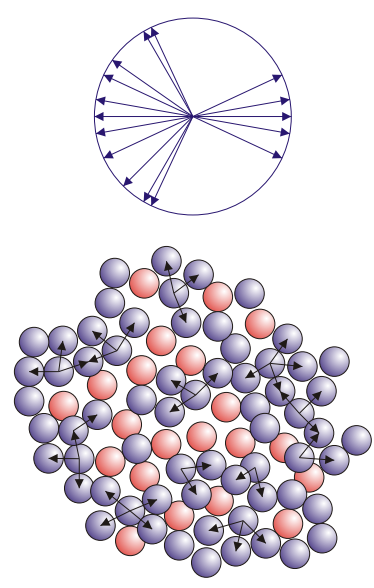

(b)

Figure 4.6. Schematic representation of the atomic pair correlation in a two-elements amorphous alloy ( $\bullet$ magnetic element, $\bigcirc$ non-magnetic) with isotropic correlation (a) and directional order (b). The distribution of moments directions indicates the resultant anisotropy orientation.

proposed by Harris, Pliske and Zuckermann $[72]$ (random axial anisotropy model) for the description of an amorphous system characterized by pair correlation and a simplified contour energy as that shown in figure 4.5 (c).

\subsection{Collective Magnetic Order in Disordered Materials}

As shown in the previous section, structural and chemical disorder may have direct consequences on the local magnetic properties of a system: variations in strength of the exchange interactions, site-dependent magnitude of the magnetic moments, and direction distributions of the magnetic anisotropy. Nevertheless disordered materials show collective orders (e.g., ferromagnetism) as perfect crystals do. Theoretically Gubanov demonstrated that ferromagnetic order exists in any material independently from its amorphous or crystalline nature [67], provided that the exchange constant $J$ remains positive, the concentration of magnetic atoms exceeds the percolation threshold, and random anisotropy is small compared to exchange coupling (see section 4.3) [126]. In principle, there are no 
fundamental differences between crystalline and amorphous ferromagnets since chemical or structural disorder does not destroy the ferromagnetic ground state.

Experimentally, however, the ferromagnetic ground state of a disordered material may be not unique or the state with lowest energy, and several interesting phenomena have therefore been observed: the phase transition at the Curie temperature may be smeared out by disorder, magnetic and non-magnetic atoms of the same element can sometimes coexist in the ordered phase, and bulk anisotropy tends to be less in amorphous system then in corresponding crystalline ones. If the ferromagnetic state is not the state with the lowest energy, than the classical collinear ferromagnetism is substituted by a non-collinear order (or random order) in magnetic amorphous solids. The different possible moments distributions at $T=0 \mathrm{~K}$ in non-crystalline materials having only one ensemble ("subnetwork") of magnetic atoms are schematically illustrated in figure 4.7. The subnetwork (a)

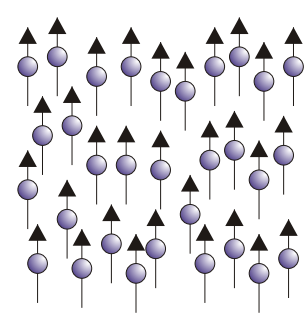

(a) ferromagnet

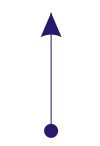

$\mathrm{M}=\mathrm{M}_{\max }$

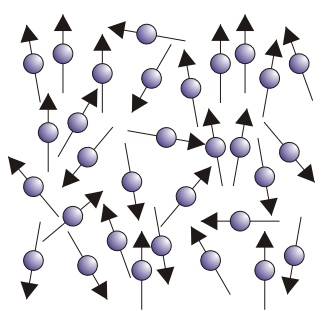

(b) speromagnet

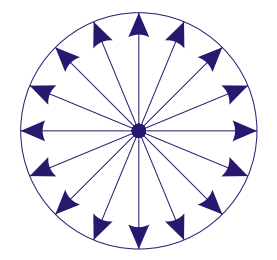

$\mathrm{M}=0$

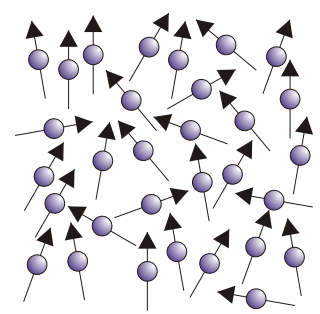

(c) asperomagnet

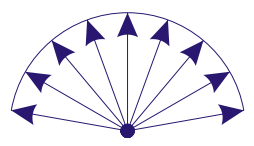

$\mathrm{M}<\mathrm{M}_{\max }$

Figure 4.7. Schematic one-subnetwork magnetic structures in amorphous solids showing the spatial distribution of moments directions and angles in the case of a ferromagnet (a), a speromagnet (b), and an asperomagnet (c).

represents a collinear structure having the maximum net moment and is identified as ferromagnetic order. The subnetworks (b) and (c) indicate random non-collinear structures showing, respectively, zero (speromagnetic) and substantial net magnetization (asperomagnetic).

The definition of a magnetically ordered structure is however valid only within a "domain" (within a characteristic length scale $L$ ) since the averaged spin correlation in any material is usually zero in zero magnetic field. Hence, if we define a correlation function as the average

$$
\left\langle\boldsymbol{S}_{i} \cdot \boldsymbol{S}_{j}\right\rangle_{R}
$$

over every moment pair $(i, j)$ for which $r_{i, j} \leq R$, the characteristic correlation length scale 
(or domain size) is that distance $L$ for which

$$
\begin{gathered}
\left\langle\boldsymbol{S}_{i} \cdot \boldsymbol{S}_{j}\right\rangle_{R}=0 \quad \text { if } R>L \\
\left\langle\frac{\boldsymbol{S}_{i} \cdot \boldsymbol{S}_{j}}{\left|\boldsymbol{S}_{i}\right| \cdot\left|\boldsymbol{S}_{j}\right|}\right\rangle_{R}=1 \quad \text { if } R<L
\end{gathered}
$$

In a speromagnet the concepts of domain or domain size has no obvious meaning while for an asperomagnet it can still be well defined as the distance $L$ for which

$$
\begin{aligned}
& \left\langle\frac{\boldsymbol{S}_{i} \cdot \boldsymbol{S}_{j}}{\left|\boldsymbol{S}_{i}\right| \cdot\left|\boldsymbol{S}_{j}\right|}\right\rangle_{R} \rightarrow 0 \text { for } R \gg L \\
& \left\langle\frac{\boldsymbol{S}_{i} \cdot \boldsymbol{S}_{j}}{\left|\boldsymbol{S}_{i}\right| \cdot\left|\boldsymbol{S}_{j}\right|}\right\rangle_{R} \leq 1 \text { for } R<L
\end{aligned}
$$

The correlation length $L$ expresses, in analogy with the concept of the average domain size in a ferromagnetic system, the mean size of regions with magnetically correlated non-collinear spins.

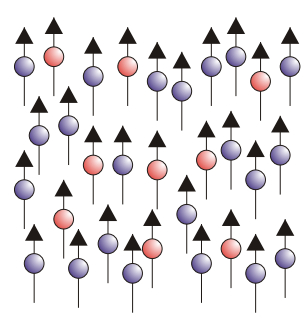

(a) ferromagnet

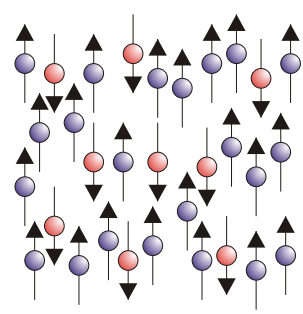

(b) ferrimagnet

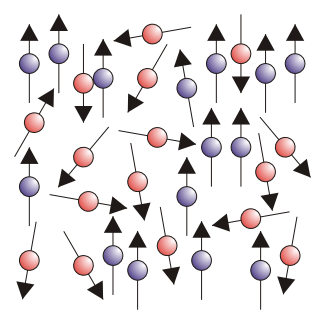

(c) sperimagnets

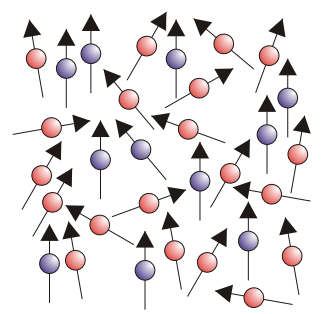

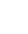
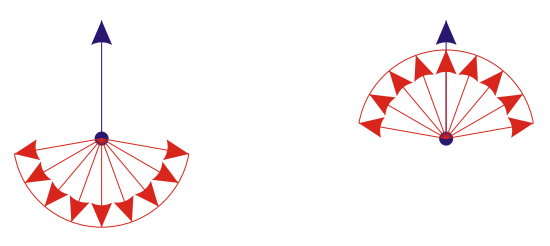

$\mathrm{M} \neq 0\left(<\mathrm{M}_{\max }\right)$

Figure 4.8. Possible two-subnetwork magnetic structures and moments spatial distribution in amorphous solids with ferromagnetic (a), ferrimagnetic (b), or sperimagnetic order (c).

All the different magnetic structures shown in figure 4.7 have direct experimental evidence, however most of the amorphous magnetic materials are multicomponent systems with at least two magnetic subnetworks. The most known examples are the family of $\mathrm{RE}-\mathrm{TM}$ rare earth and transition metals glasses: $\mathrm{Nd}-\mathrm{Fe}-(\mathrm{Al}, \mathrm{B}, \ldots)$ or $(\mathrm{Gd}, \mathrm{Pr}, \mathrm{Nd}, \mathrm{Sm})-$ (Fe, Co), for which two subnetworks must be distinguished. For those systems, collinear structures are ferromagnetic, if the net magnetization is maximal $\left(M=M_{\max }\right)$ and ferrimagnetic, if $M<M_{\max }$, while non-collinear are conventionally defined sperimagnetic, if $M \neq 0\left(<M_{\max }\right)$, and speromagnetic, if $M \neq 0$ (see figure 4.8). 


\subsection{Random Axial Anisotropy Model (RAM)}

The random axial anisotropy (RAM) model is one of the most successful theoretical description for magnetism in disordered systems with asperomagnetic order and, although highly simplified, it is still a point of reference for understanding the magnetic properties of amorphous alloys containing rare-earth ions [41].

The Hamiltonian proposed by Harris, Plishke, and Zuckermann 72 to account for anisotropy directions varying randomly from an atomic site to an other is composed of three terms representing the exchange energy, the dipolar interaction with an externally applied field $H$ (Zeeman energy), and the random anisotropy energy

$$
\begin{aligned}
\mathscr{H}^{R A M} & =\mathscr{H}^{E x}+\mathscr{H}^{D i p}+\mathscr{H}^{A n}= \\
& =-\frac{1}{2} \sum_{i \neq j} J_{i j} \boldsymbol{S}_{i} \cdot \boldsymbol{S}_{j}-\sum_{i} g \mu_{B} \boldsymbol{S}_{i} \cdot \boldsymbol{H}-\sum_{i} K_{i}\left(\boldsymbol{n}_{i} \cdot \boldsymbol{S}_{i}\right)^{2}
\end{aligned}
$$

where $\boldsymbol{S}_{i}$ is the spin at the site $i, g \mu_{B} S_{i}$ its magnetic moment, $K_{i}$ the anisotropy constant, $\boldsymbol{n}_{i}$ the anisotropy vector, and $J_{i j}$ the exchange constant between the spins $\boldsymbol{S}_{i}$ and $\boldsymbol{S}_{j}$.

If we assume that the exchange interaction is proportional to an internal field $H_{m}$ (molecular field approximation) directed along $H$ ( $z$ direction), then

$$
\frac{1}{2} \sum_{i \neq j} J_{i j} \boldsymbol{S}_{i} \cdot \boldsymbol{S}_{j} \simeq \sum_{i} g \mu_{B} S_{i z} H_{m}
$$

where $S_{i z}$ is the projection of $\boldsymbol{S}_{i}$ along $z$ and

$$
H_{m}=\lambda\left\langle\frac{S_{j z}}{S_{j}}\right\rangle_{N_{i}}
$$

where $\lambda$ is the molecular field constant and the average $\left\langle S_{j z} / S_{j}\right\rangle$ is evaluated over $N_{i}$ nearest neighbors of the atom $i$. Thus equation 4.9 can be rewritten as

$$
\mathscr{H}^{R A M}=-\sum_{i} g \mu_{B} S_{i z}\left(H+H_{m}\right)-\sum_{i} K_{i}\left(\boldsymbol{n}_{i} \cdot \boldsymbol{S}_{i}\right)^{2}
$$

where $\left(H+H_{m}\right)$ represents an effective magnetic field $H^{*}$ acting on each spin.

For an ideal situation in which exchange interaction is zero $\left(H_{m}=0\right)$ and $K_{i}$ is constant at each atomic site $i$, the reduced magnetization curve $\left(S_{i z} / S_{i}\right)$ for any atom can be calculated from the relation

$$
\mathscr{H}_{i}=-g \mu_{B} S_{i z} H-K\left(\boldsymbol{n}_{i} \cdot \boldsymbol{S}_{i}\right)^{2}
$$

or, from the classical case [29], by minimizing the energy

$$
E_{i}=-\mu H \cos \left(\phi_{i}\right)-K \cos ^{2}\left(\theta_{i}-\phi_{i}\right)
$$




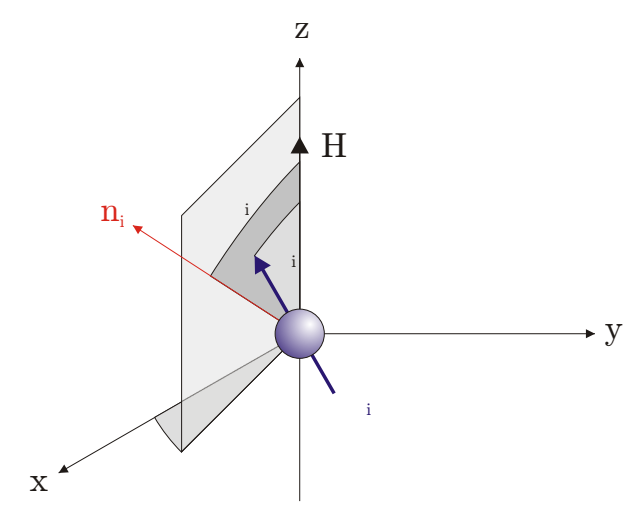

Figure 4.9. Relative orientations of magnetic field $H$, local anisotropy $\boldsymbol{n}_{i}$ and magnetic moment $\mu_{i}$ at the $i$-th atomic site.

where $\phi_{i}$ and $\theta_{i}$ are defined in figure 4.9 and $\mu$ is the magnetic moment on each atom $\left(\mu=g \mu_{B} S\right)$. The minimum energy results for $\phi_{i}$ given by the relation

$$
\frac{d E_{i}}{d \phi_{i}}=\mu H \sin \left(\phi_{i}\right)-2 K \sin \left(\theta_{i}-\phi_{i}\right) \cos \left(\theta_{i}-\phi_{i}\right)=0
$$

and, hence

$$
\phi_{i}=f\left(\theta_{i}, K, H\right)
$$

The reduced magnetization curve can be calculated after averaging over all the possible orientations of $\boldsymbol{n}_{i}$ as

$$
\begin{aligned}
\frac{M}{M_{\text {max }}}=m=\frac{\left\langle\mu_{z}\right\rangle}{\mu} & =\frac{\left\langle\mu \cos \left(\phi_{i}\right)\right\rangle}{\mu}=\frac{\int \cos \left(\phi_{i}\right) d \boldsymbol{r}}{\int d \boldsymbol{r}}=\frac{\iint \cos \left(\phi_{i}\right) r^{2} \sin (\theta) d r d \varphi d \theta}{\iiint r^{2} \sin (\theta) d r d \varphi d \theta}= \\
& =\frac{\int \cos \left(\phi_{i}\right) \sin (\theta) d \theta}{\int \sin (\theta) d \theta}=\int_{0}^{\pi / 2} \cos \left(\phi_{i}\right) \sin (\theta) d \theta
\end{aligned}
$$

and shows a dependence from the external magnetic field as presented in figure 4.10 .

From equation 4.15 results also that, for $H \simeq 0, \phi_{i}$ is almost equal to $\theta_{i}$ and, hence

$$
\left.m\right|_{H=0}=m_{r}=\frac{1}{2}
$$

The magnetic structure in zero applied field is therefore asperomagnetic and the hemisphere, within which the moments were oriented, narrows to a cone around the positive $z$ axis as $H$ increases. Large fields are needed to achieve complete saturation ${ }^{(1)}$ as it can be deduced from the field dependence of the magnetization for high values of $H$

$$
\left.m\right|_{H \gg 0} \propto \frac{1}{H^{2}}
$$

${ }^{(1)}$ For $\mathrm{Dy}^{3+}, K$ is approximately $0.517 \mathrm{meV}$ and the ions would need an applied field of $1.2 \mathrm{MOe}$ $(\sim 120 \mathrm{~T})$ to reach $m=0.95$. 


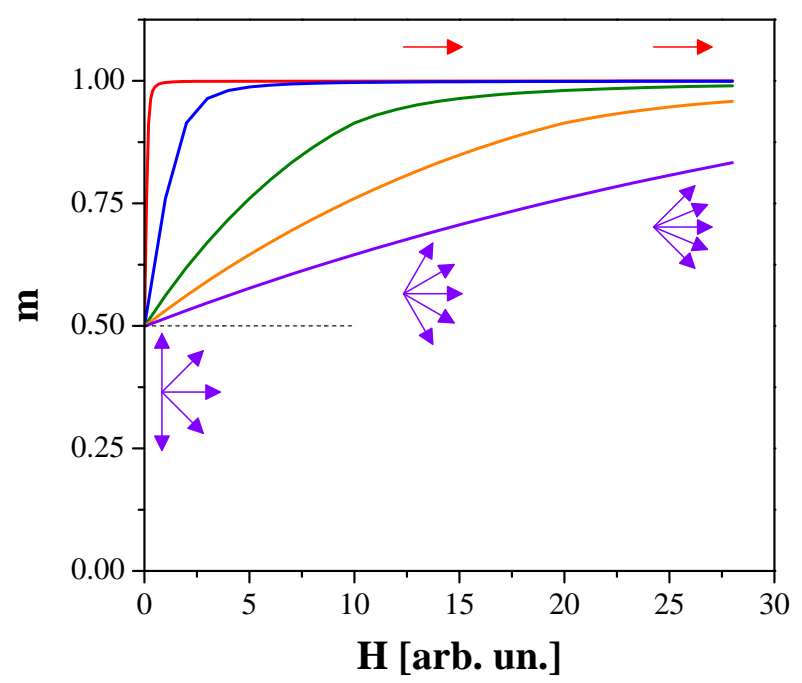

Figure 4.10. Reduced magnetization curves, calculated from equation 4.15 and 4.17 for an array of non-interacting magnetic ions with random easy-axis anisotropy and anisotropy constant (expressed in arbitrary units) equal to $0.1(-), 1(-), 5(-), 10(-)$, and $20(-)$, respectively.

If the exchange interaction is not zero, the effective field acting on each spin becomes, in the molecular field approximation, $H^{*}=H+H_{m}$ and the reduced moment $m$ increases with the magnitude of the exchange interaction. Figure 4.11 shows qualitatively the calculated variation of $m_{r}$ as function of the ratio $\alpha$ between anisotropy and exchange constants at $T=0 \mathrm{~K}$

$$
\alpha=\frac{K}{\mu \lambda}
$$

For $K \gg J$, the collective order of the system becomes asperomagnetic while, for $K \ll J$ the system is a perfect ferromagnet with small saturation field $\left(m_{r} \simeq 1\right.$ for $H \simeq 0$ ).

In the same way as the remanence state has been calculated, also the hysteresis behavior of an array of spins can be deduced as function of different values of anisotropy and exchange constants [29. Some hysteresis loops at $T=0 \mathrm{~K}$ are shown for example in figure 4.12. Such calculations indicate that there is a threshold value of anisotropy constant $\left(K^{\prime}=0.6 \cdot \mu \lambda\right)$ where coercivity appears. For $K>K^{\prime}$, the coercivity $H_{c}$ results to be proportional to

$$
H_{c} \simeq \frac{K}{\mu}
$$

which follows whenever the moments are unable to overcome the anisotropy barriers separating metastable states (in the second and fourth quadrants of the hysteresis loop) from the stable ground states. 


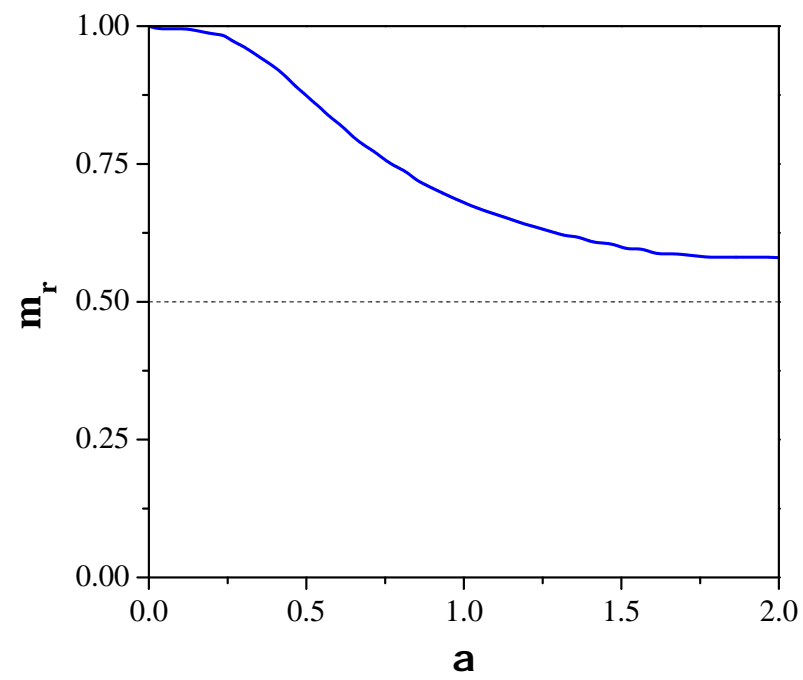

Figure 4.11. Reduced remanence $m_{r}$ as function of the ratio $\alpha$ between anisotropy and magnetic exchange energy 29$]$.

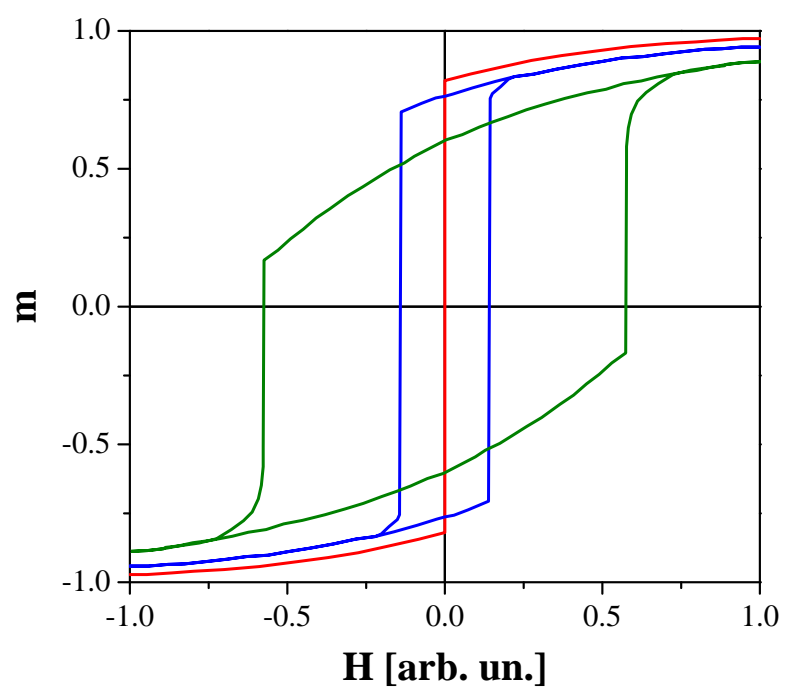

Figure 4.12. Calculated hysteresis loops at $T=0 \mathrm{~K}$ in the molecular field approximation for several ranges of the ratio $\alpha$ between anisotropy and magnetic exchange energy: $0 \leq$ $\alpha<0.6(-), 0.6 \leq \alpha<1.0(-)$, and $1.0 \leq \alpha<5.3(-)$, respectively $[29$. 


\subsubsection{Magnetic Domains}

In crystalline ferromagnets, the collective magnetic order generally breaks up into welldefined regions (magnetic domains) under the influence of dipolar fields and magnetocrystalline anisotropy. The molecular field theory can only be applied in those systems to the behavior of a single domain, but not to the entire sample. Similarly, it is unlikely that an uniform asperomagnetic (or sperimagnetic) state is actually the state of lowest energy for a system with random axial anisotropy in the absence of an external magnetic field. Therefore, the problem of the magnetic configuration of non-collinear disordered systems reduces basically to the definition of a correlation length $L$ (see section 4.2 ) within which the RAM model in the mean field approximation can be still applied.

The correlation direction or local $z$-axis will vary at random over a length scale which depends on the range of the interaction or, equivalently, on the number of interacting neighbors. Figure 4.13 illustrates this problem for a row of spins, in the large $\alpha$ limit (high anisotropy), which have: (a) random orientations (speromagnetic state), (b) 2neighbor exchange interaction, (c) 8-neighbors exchange interaction, and (d) long range interaction (uniform asperomagnetic state). The latter configuration is expected to be

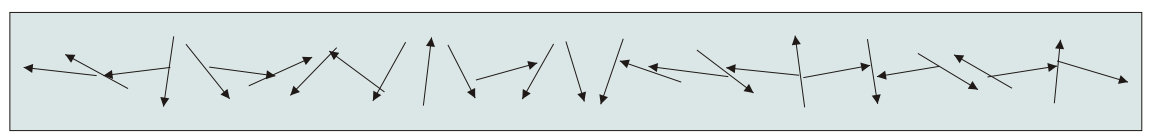

(a) random orientation

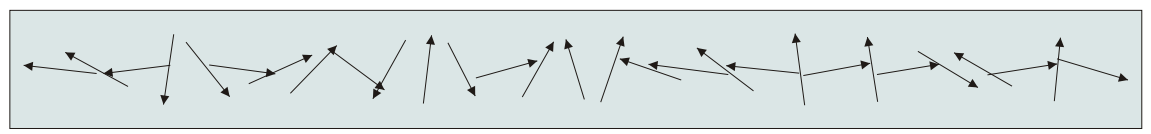

(b) 2-neighbors interaction

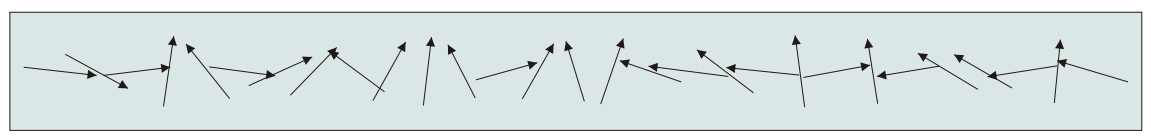

(c) 8-neighbors interaction

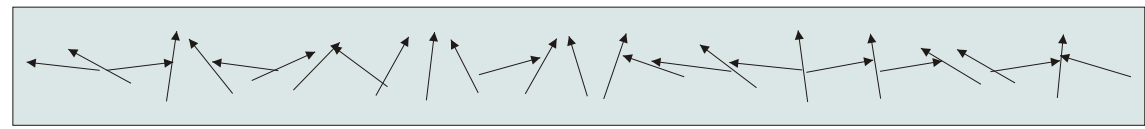

(d) upward direction

Figure 4.13. Different magnetic configurations for a row of spins with equal random anisotropy directions in the case of: (a) no neighbor exchange interaction, (b) 2-neighbors interaction, (c) 8-neighbors interaction, and (d) long range interaction.

marginally higher in energy than (b) or (c), however, a state of slightly lower energy can be obtained if the $z$ direction is allowed to respond to the random local fluctuations in the correlations of the anisotropy axes. In one dimension, weak points occur where the easy axes on adjacent sites are nearly perpendicular, and they delimit small asperomagnetic "domains" which are caused by minimization of the exchange interactions. 
In the limit $K \gg J(\alpha \gg 1)$, where the magnetic moments are strongly pinned to anisotropy axes (figure 4.13 (b) or (c)), the correlation length is approximatively 42

$$
L_{c o r r} \simeq N a
$$

where $N$ is the number of interacting neighbors and $a$ is the mean interatomic distance. Hence, the local magnetization direction varies on a length scale comparable to the interatomic spacing in a small asperomagnetic domain where the $z$-axis is defined locally as the direction of the exchange field $H_{m}$ acting on a central atom. The opposite limit, $K \ll J(\alpha \sim 0)$, is the case of strongly correlated magnetic moments and anisotropy which produces only small deviations from local ferromagnetic alignment.

If we assume that the spin directions are correlated within a region of size $L_{c o r r}$, the number of interacting spins in a domain is proportional to

$$
n \simeq\left(\frac{L_{\text {corr }}}{a}\right)^{3}
$$

The anisotropy energy arising from local deviations within a domain results to be [2]

$$
E_{A n} \simeq-\frac{K}{\sqrt{n}}=-K\left(\frac{a}{L_{\text {corr }}}\right)^{3 / 2}
$$

and the exchange energy, on passing from one domain to the next (where the magnetic axis rotates by $\pi / 2$ over a distance of order $L_{\text {corr }}$ ), becomes

$$
E_{E x} \simeq A\left(\frac{\pi}{2} \frac{a}{L_{\text {corr }}}\right)^{2}
$$

where $A$ is the magnetic stiffness. The value of $L_{c o r r}$ which gives the minimum total energy $\left(E_{A n}+E_{E x}\right)$ is then

$$
L_{\text {corr }}=\frac{16}{9} \frac{A^{2}}{K^{2} a^{3}}
$$

This result shows that local anisotropy never averages out completely, but if $K / J$ is small, then its effect is greatly reduced by averaging and an effective anisotropy $K_{\text {eff }}$ $(=K / \sqrt{n})$ characterizes instead the system. Nevertheless, a true ferromagnetic state is always destabilized by random anisotropy, although the ferromagnetic correlation length $L_{\text {corr }}$ may be so long that the magnetic properties of a material possessing weak random anisotropy are practically indistinguishable from those of a ferromagnet.

In conclusion, when $K \sim 0$, domains in one-network amorphous ferromagnets should be similar to those in crystalline samples with the same shape and bulk magnetic properties. As $K / J$ increases (while remaining positive), one passes over from ferromagnetic to an asperomagnetic structure whose domain size will depend sensitively on the range of the ferromagnetic exchange interaction and, in the limit of nearest-neighbor interactions, domains become extremely small [42]. On the contrary, the concept of domain is lost in speromagnets in which the spin direction changes randomly from site to site. 


\subsection{Magnetization Mechanisms}

The magnetization mechanisms determining the shape of the hysteresis curve of a magnetic material cannot be limited to magnetization rotation, as implicity presupposed by the random axial anisotropy model (see section 4.3). In fact, the processes which characterize the hysteretic behavior of a ferromagnet are multiple and are generally classified in four broad group: ${ }^{(2)}$ magnetization rotation, domain wall motion, (domain) nucleation, and topological hysteresis. Each mechanism is characterized by a set of phenomena that may give some fundamental indications to the interpretation of the experimental results. Nevertheless, a detailed description of each class is left to some more appropriate references 12, 85 and only a qualitative aspect will be here taken into account. An important feature associated to the role of nucleation and domain walls motion (i.e., domain wall pinning) in the hysteretic behavior of a hard magnetic material can be recognized by comparing its virgin magnetization curve with the saturation branch of the hysteresis loop (figure 4.14). If the virgin curve is steep and reaches the saturation under fields much
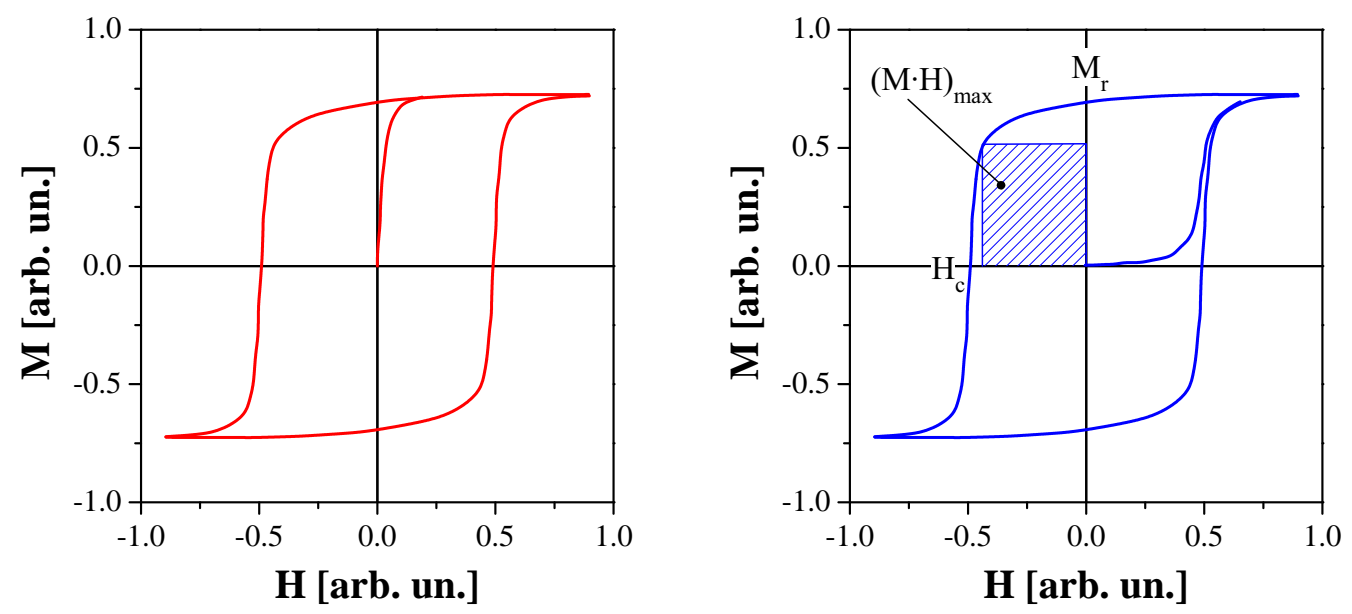

Figure 4.14. Schematic representation of magnetization curves in nucleation-type $(-)$ and pinning-type $(-)$ magnets.

lower than the coercive field $H_{c}$, one accordingly speaks of nucleation-type magnets. Domain walls are present in the virgin state and the fact that the material can be easily saturated shows that walls are free to move and do not experience pinning effects. Once the virgin domain structure has been removed upon application of high fields, the formation of reversed domains becomes difficult and the demagnetization curve is characterized by a substantial coercivity. On the contrary, if fields of the order of $H_{c}$ are required to saturate the material also in the virgin curve, one speaks of pinning-type magnets and

\footnotetext{
${ }^{(2)}$ This classification is usually proposed to roughly explain the shape of the hysteresis loops at intermediate fields, between the regions of low fields (Rayleigh region) and of large fields (approach to saturation) in which, under certain conditions, magnetization curves can be described by analytical expressions.
} 
domain wall pinning is the main mechanism responsible for the coercivity 69.

Due to the importance of domain configuration and domain-wall pinning in the magnetic behavior of heterogenous materials and intrinsic composites [48], the following section is dedicated to the description of coercivity mechanisms and the theoretical model proposed by Gaunt to account for the pinning-type magnetic behavior of RE-TM alloys 63 .

\subsection{Coercivity Mechanisms}

The term coercivity indicates generally the strength of any external action which is necessary to change the initial state of a system. In the specific case of a magnetic material the coercivity, or better the coercive field $H_{c}$, is the field at which the magnetization is reduced from remanence to zero $\left[12\right.$ and, hence, $H_{c}$ is a measure of the resistance of a sample to the reversal of the magnetization upon application of an increasing external magnetic field $H$.

The concept of magnetic coercivity is of great technological as well as physical importance. Technologically the coercivity is connected to the use of the terms "soft" and "hard" which define the two broad classes of ferromagnetic materials suitable for applications. The energy dissipated (as heat) by a ferromagnet during a hysteresis loop is proportional to the area of that loop and it can be estimated by the product $4 \mu_{0} M_{\max } H_{c}$ where $M_{\max }$ is the so-called maximum magnetization which gives the maximum $(M \cdot H)_{\max }$ product (see figure 4.14). If the amount of energy dissipated in a hysteresis cycle is small (or if $H_{c}$ is low), the material is said to be magnetically soft while if it is large (or if $H_{c}$ is high), the material is said to be magnetically hard. Physically it is already well known that coercive field values span an astonishingly wide interval which goes from less than $1 \mathrm{~A} / \mathrm{m}$ in very soft $\mathrm{Ni}-\mathrm{Fe}$ or amorphous alloys to $10^{7} \mathrm{~A} / \mathrm{m}$ in recent hard magnets. There is a great variety of chemical ("doping"), metallurgical, geometrical (shape anisotropy effects), and structural (microstructural length scales, pinning effects, defects, etc.) factors that may substantially affect the coercive field of a magnetic specimen. Therefore, coercivity (and, as consequence, the hysteresis loop) is not an intrinsic property of individual volumes of a given element or alloy but a physical parameter which, measuring the typical strength of the energy gradients that must be overcome in order to achieve substantial magnetization changes in a material, is directly linked to the mechanisms governing the magnetization processes. An example of material dependency of the coercive field $H_{c}$ is reported in table 4.1 for the case of different types of pure Fe samples.

Coercivity mechanisms are schematically divided into two broad classes on the basis of the process which contributes mainly to the magnetization reversal and which can only be of two types:

- magnetization rotation 


\begin{tabular}{cc}
\hline Sample & $H_{c}[\mathrm{~A} / \mathrm{m}]$ \\
\hline very pure bulk Fe & 1 \\
commercial polycrystalline Fe & 100 \\
single domain Fe particle & 10000 \\
\hline
\end{tabular}

Table 4.1. Coercivity field $H_{c}$ for different types of pure Fe samples.

- domain wall motion (i.e., domain wall pinning)

The fundamental difference which distinguishes the two classes is the energy necessary to force the magnetization reversal. In the case of magnetization rotation in a certain volume $V$, energy has to be supplied to average out the anisotropy to the whole volume and the expression of the coercive field becomes [12,13]:

$$
H_{c}=\frac{2 K}{\mu_{0} M_{s}}
$$

where $K$ is the anisotropy constant (or its effective value, see section 4.3.1) and $M_{s}$ is the saturation magnetization. Equation 4.27 is in accordance with the expression of the coercivity given by the random anisotropy model in equation 4.21. In the case of domain wall motion, on the contrary, only the energy stored in the wall thickness is involved and it is often weakly dependent on wall position if the system is ideally a homogeneous defect-free magnet. In heterogeneous systems pinning effects may play an important role.

\subsubsection{Domain Wall Pinning}

Pinning processes first requires the nucleation of a reverse domain. In some cases, the field needed to produce a reversed domain (nucleation field) is larger than the field necessary to expand it and, hence, the domain walls can move freely throughout the sample without effectively experiencing any impediments. However, if the system is not fully homogeneous, several causes may be responsible for pinning of the domain walls (see section 4.5.1.2 and coercivity results to be strongly affected.

Many models have considered the different origins of pinning and deduced the relative expression of the coercivity field as function of microstructural parameters: Kersten (in the case of non-magnetic inclusions and residual stresses [37,89]), Néel [85], and Gaunt (in the case of defects and precipitates [63]), Kronmüller (in the case of magnetic pinning sites 98 ), and many other 12,85 .

Among them, the description of the domain wall pinning proposed by Gaunt is of great interest in particular for RE-TM systems $48,63,69$. 


\subsubsection{Gaunt's Model}

Gaunt considered the close analogy between the coercivity of ferromagnetic materials containing non-magnetic inclusions and the yield stress in two-phase alloys and based his model on the Friedel model of the pinning of dislocations at precipitates.

A fundamental approximation of most models developed to explain the unpinning process of the domain wall at pinning site consists in considering the wall as a deformable membrane of surface energy $\gamma_{w}$ per unit area pinned, and to reduce the rather complex statistical problem of a random array of pins to consideration of the forces required to move the wall over a "unit step".

The early model of Kersten [89] expressed the coercive field as consequence of a single obstacle interacting with an area $A_{w}$ of the wall by the relation

$$
H_{c}=\frac{1}{2 \mu_{0} M_{s} A_{w}}\left(\frac{d U}{d z}\right)=\frac{f}{2 \mu_{0} M_{s} A_{w}}
$$

where $M_{s}$ is the saturation magnetization and $(d U / d z)_{\max }=f$ is the maximum restoring force which a pin of interacting energy $U(z)$, can exert on wall forced to move in the direction $z$ by an external magnetic field $H$. To solve the pinning problem for a particular material, it is therefore necessary to know the interaction potential $U(z)$, and its derivative, and to determine the "unit step". Two models have been proposed by Gaunt: strong pinning and weak pinning. The difference between them consists in the fact that weak pinning is a cooperative effects in which a domain wall breaks away simultaneously from several pinning centers, whereas in the strong pinning case, the wall bows out from one pin at a time.

\section{Strong Pinning}

Consider a domain wall parallel to the $x y$ plane interacting with an array of pinning sites of volume density $\rho$ as schematically presented in figure 4.15. On applying a field $H$, parallel to the magnetization direction $x$, the wall will bow out and, when $H$ increases sufficiently, will break away from the center pin and then from the outer square of obstacles.

Assuming an area per obstacle $A_{w}=\pi L_{1}^{2}$, it results (see figure 4.16)

$$
2 R h_{1}=L_{1}^{2}
$$

where $R$ is the radius of curvature of the bowed wall and $h_{1}\left(h_{1} \ll R\right)$ is the maximum displacement of the wall before breakaway. The volume swept out by the field per obstacle is

$$
V_{1}=\pi h_{1}^{2} R
$$

and after breakaway

$$
V_{2}=\pi h_{2}^{2} R
$$




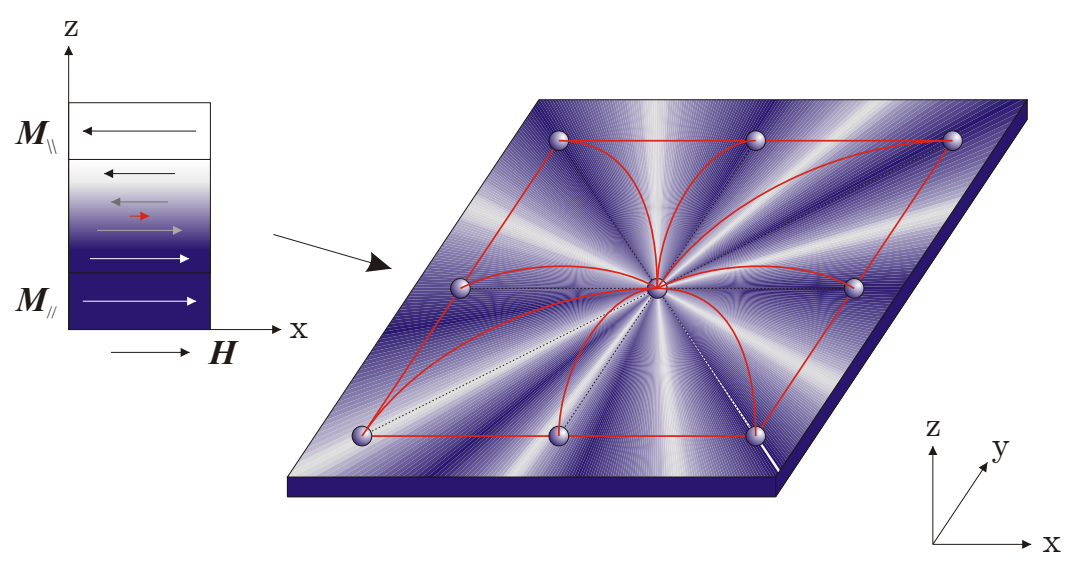

Figure 4.15. $180^{\circ}$ domain wall pinned in $x-y$ plane. The broken lines $(-)$ represent the wall in the initial position $(H=0)$ while the solid lines indicate bowing when a field is in $z$ direction (-).

from which it results a total volume difference between breakaway and establishment of a new steady state regime

$$
\Delta V=V_{1}-V_{2}=\frac{3 \pi L_{1}^{4}}{R}
$$

but surface tension theory gives 63

$$
\mu_{0} M_{s} H=\frac{\gamma_{w}}{R}
$$

thus equation 4.32 becomes

$$
\Delta V=\frac{3 \pi L_{1}^{4} \mu_{0} M_{s} H}{\gamma_{w}}
$$

The steady-state criterion for dislocation pinning (Friedel criterion) requires that

$$
\rho \Delta V=1
$$

where $\rho$ is the pin density per unit volume. In this case, the wall sweeps out a volume containing on average one pin, and

$$
3 \pi L_{1}^{4} \mu_{0} M_{s} H \rho=\gamma_{w}
$$

from which it results

$$
A_{w}=\pi L_{1}^{2}=\left(\frac{\pi \gamma_{w}}{3 \mu_{0} M_{s} H \rho}\right)^{1 / 2}
$$

Thus, equation 4.28 expresses the coercive field at $0 \mathrm{~K}$ as

$$
H_{c}^{0 \mathrm{~K}}=H_{0}=\frac{3 \rho f^{2}}{4 \pi \gamma_{w} \mu_{0} M_{s}}
$$

The model proposed by Gaunt breaks down if the mean area per pin before applying the field $\left(A_{w 0}\right)$ is already less than the area after the application of the field $\left(A_{w}\right) . A_{w 0}$ is 


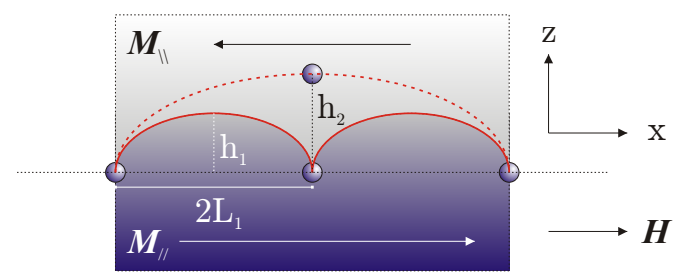

Figure 4.16. Cross-section of a bowed domain wall in a external magnetic field. The broken line (-) represent the wall for $H=0$ while the solid line $(-)$ show the wall after breakaway from the central pin.

defined by the relation

$$
A_{w 0}=\frac{1}{4 b \rho}
$$

where $4 b$ is the pin interaction length, thus for $H=H_{c}$ the Gaunt model is applicable if

$$
\frac{1}{4 b \rho}=\left(\frac{\pi \gamma_{w}}{3 \mu_{0} M_{s} H \rho}\right)^{1 / 2}
$$

or:

$$
\left(\frac{3 f}{2 \pi \gamma_{w} 4 b}\right)^{1 / 2}=\beta>1
$$

Thus the pinning is strong for large $f / \gamma_{w}$ or for small $b^{(3)}$.

\section{Thermal Activation Effects}

The above presented theory calculates the coercive force at $T=0 \mathrm{~K}$, neglecting therefore thermal activation effects. At $T \neq 0 \mathrm{~K}$, the wall can in fact break free in a field $H<H_{c}$ since the energy necessary to bow the wall away is supplied by thermal energy. Thus the wall will move from a minimum energy position to the next one overcoming an energy barrier $\Delta E$ (see figure 4.17 ).

Supposing that a domain wall moving in the $x$ direction interacts with an inhomogeneity, the total energy can be written as

$$
E=U(x)-2 \mu_{0} M_{s} H A_{w} x
$$

where $U(x)$ is the interaction energy and $x$ is the wall displacement. If the interaction force $f=d U / d x$ is symmetrical at $x=0$ then $U(x)$ can be expressed as

$$
U(x)=a_{0}+a_{1} x+a_{3} x^{3}+\ldots
$$

and, usually, the following interaction energy is suggested as a general form

$$
U(x)=f\left(a+x-\frac{x^{3}}{3 b^{2}}\right)
$$

${ }^{(3)}$ The minimum value of $b$ is, even for "point pins" of negligible diameter, $t / 4$ where $t$ is the domain wall thickness. 


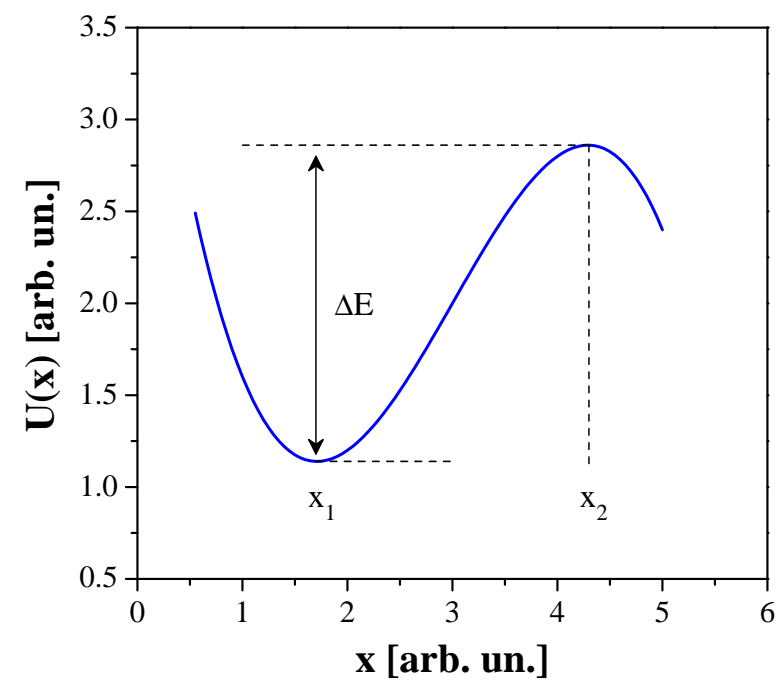

Figure 4.17. Schematic representation of the potential $U(x)$ experienced by a domain wall affected by pinning mechanism. $\Delta E$ represents the energy barrier which the domain wall must overcome for moving from one pinning site to the other.

where $a$ is a constant and $b$ is proportional to the pin interaction length $(4 b)$. The equilibrium wall position can be found differentiating equation 4.42 with $U(x)$ expressed as in equation 4.44

$$
\frac{\partial E}{\partial x}=\left(f-2 \mu_{0} M_{s} H A_{w}\right)-f \frac{x^{2}}{b^{2}}=0
$$

which gives

$$
x_{1,2}= \pm\left(1-\frac{2 \mu_{0} M_{s} H A_{w}}{f}\right)^{1 / 2}
$$

The negative sign corresponds to an energy minimum, the positive to an energy maximum , and the difference represents an activation energy which may be overcome by thermal activated processes at high enough temperatures. The activation energy is therefore 64

$$
\Delta E=E\left(x_{2}\right)-E\left(x_{1}\right)=4 b \frac{\left(f-2 \mu_{0} M_{s} H A_{w}\right)^{3 / 2}}{3 f^{1 / 2}}
$$

According to equation 4.28 and expressing the pin force as $f=2 \mu_{0} M_{s} H_{0} A_{w}$, the previous relation becomes

$$
\Delta E=\frac{4}{3} b f\left[1-\left(\frac{H}{H_{0}}\right)^{1 / 2}\right]^{3 / 2}
$$

In the case of thermal activation, the energy $\Delta E$ can also be expressed though the relation

$$
\Delta E=c \cdot k_{B} T
$$

where $c$ is a constant ${ }^{(4)}$ and $k_{B}$ is the Boltzmann's constant. Thus the coercive field $H_{c}(T)$

\footnotetext{
${ }^{(4)}$ According to magnetic viscosity theory $\left[12, E=25 k_{B} T\right.$ has become a customary and a convenient estimate of the energy scale involved in common hysteresis and relaxation experiments.
} 
will follow the equation

$$
\left(\frac{H_{c}}{H_{0}}\right)^{1 / 2}=1-\left(\frac{75 k_{B} T}{4 b f}\right)^{2 / 3}
$$

If demagnetization effects of the bowed wall are included in the development of the model a result similar to equation 4.50 can be found

$$
\left(\frac{H_{c}}{H_{0}}\right)^{3 / 7}=1-\left(\frac{75 k_{B} T}{4 b f}\right)^{2 / 3}
$$

Equation 4.50 can be expressed independently from the values of the interaction length $4 b$ and of the force per pin $f$ defining a characteristic temperature $T_{0}$ as

$$
T_{0}=\frac{4 b f}{75 k_{B}}
$$

which represents the temperature at which the pinning effects are completely overcome by thermal effects. In fact, for $T>T_{0}$ the thermal energy is large enough to average out the energy barriers due to the random distribution of pinning centers which cannot, therefore, exert any force on the domain walls and a zero coercivity results. Introducing the previous relation in equation 4.50 , the temperature dependence of the coercive force in an inhomogeneous magnetic system characterized by pinning centers can be expressed by the relation

$$
\left(\frac{H_{c}}{H_{0}}\right)^{1 / 2}=1-\left(\frac{T}{T_{0}}\right)^{2 / 3}
$$

which will be used in the following for the interpretation of the magnetic properties of the $\mathrm{Nd}_{60} \mathrm{Fe}_{30} \mathrm{Al}_{10}$ samples.

\section{Weak Pinning}

Strong magnetic pinning is not the only type of pinning behavior which experimental results and the counterpart mechanical theory of pinning have shown. Recalling equation 4.41, if $\beta$ is less than unity the domain wall cannot bow out freely between pins without meeting many more pins implying that the strong pinning model cannot be use anymore to express $H_{c}$. Leaving the theoretical description to the reference [63], Gaunt proposed a second model which accounts for weak pinning $(\beta<1)$ and expresses the coercive field as

$$
H_{c}=\frac{f^{2} \rho t^{2}}{62 \mu_{0} M_{s} \gamma_{w} b^{2}}
$$

which reaches its maximum value for "point pins" $(b=t / 4)$

$$
H_{c}=\frac{8 f^{2} \rho}{31 \mu_{0} M_{s} \gamma_{w}}
$$


As for the strong pinning model, Gaunt suggested also for the weak pinning an expression of the coercive field at finite temperatures which he expressed as

$$
H_{c}=H_{0}\left(1-\frac{25 k_{B} T}{31 \gamma_{w} b^{2}}\right)
$$

As done before, the previous relation can be rewritten in term of a characteristic temperature $T_{0}^{\prime}$ which accounts for the intrinsic properties of the pins and which can be defined as

$$
T_{0}^{\prime}=\frac{31 \gamma_{w} b^{2}}{25 k_{B}}
$$

and, thus it results that

$$
\frac{H_{c}}{H_{0}}=1-\left(\frac{T}{T_{0}^{\prime}}\right)
$$

In summary, the coercive field $H_{c}^{T=0 \mathrm{~K}}$ for a random array of pins has the same form for both weak and strong pinning while thermal activation effects differ significantly for the two cases giving completely different expressions for the temperature dependence of $H_{c}$. For strong pinning, $H_{c}^{1 / 2}$ varies with $T^{2 / 3}$ while for weak pinning $H_{c}$ is linear to $T$.

\subsubsection{Origin of Pinning Effetcs}

The analysis proposed in the previous section leaves open the problem of identifying the possible causes of pinning. Theoretical models (see [85, chap. 8] and references therein), experimental results [48, and computer simulations [140 have suggested that pinning takes place whenever domain walls experience local variations of the domain-wall energy $\gamma_{w}$. Such variations give rise to an attractive/repulsive force $f$

$$
f \propto \frac{d \gamma_{w}}{d \boldsymbol{r}}
$$

where $\boldsymbol{r}$ is the spatial vector, which strongly depends on the fluctuation of the local magnetic properties of the system and gives rise to energy barriers that act as obstacles slowing the motion or even pinning the domain walls. The effectiveness of the pinning mechanisms is directly related to the structural disorder and the way how disorder affects the local magnetic properties of the system. Possible pinning sources in crystalline materials are point defects (vacancies and interstitial atoms) and dislocations. Dispersed phases with magnetic properties different from those of the matrix as well as non-magnetic inclusions or cavities may also be important. In amorphous alloys, intrinsic fluctuations of local exchange and anisotropy constants, formation of clusters of local chemical order, and even time-dependent structural rearrangements may give rise to pinning [12]. In the case of intrinsic composites, all those causes may be present simultaneously due to the presence of magnetic and non-magnetic phases as well as structural and chemical disorder, and domain wall pinning is therefore a serious candidate for explaining the coercivity mechanism of heterogeneous systems showing hard magnetic behavior. 


\section{Chapter 5}

\section{Experimental Methods and Techniques}

Chapter 5 is dedicated to the description of methods and techniques used for structural, thermodynamical, and magnetic characterizations of the $\mathrm{Nd}_{60} \mathrm{Fe}_{30} \mathrm{Al}_{10}$ samples. Beyond a brief presentation of the measurement devices which have been used, a discussion about the applied experimental methods is given and some additions to the theory described in chapter 3 and in chapter 4 are included.

The chapter starts by describing briefly the two most important devices currently used for the characterization of magnetic samples: the superconducting quantum interference device (SQUID) magnetometer in section 5.1 and the vibrating sample magnetometer (VSM) in section 5.2. Those two sections deal mainly with the physical aspects which play a fundamental role in the measuring set-up, leaving all the technological solutions adopted to make them working to more specific references. Section 5.3 deals with the small-angle neutron scattering (SANS) technique (with unpolarized and polarized neutrons) which offers the possibility to obtain information on both, structural and magnetic, properties of the samples. The characteristics of the two neutron beamlines (at the Intense Pulsed Neutron Source, Argonne National Laboratory, and at the Center for High Resolution Neutron Scattering, National Institute of Standards and Technology, Gaithersburg) used in this work to measure the small-angle patterns are only briefly described and more information can be found in their respective user's guides. High-resolution transmission electron microscopy (HRTEM), dynamical scanning calorimetry (DSC), and high-energy wide-angle $\mathrm{x}$-ray diffractometry (HE-WAXS) techniques were employed for the investigation of structural properties of the samples and their evolution after annealing or during in situ thermal treatments. Those three techniques are presented in section 5.4, 5.5, and 5.6. respectively. As for the SANS, the HE-WAXS beamline (at the Advanced Photon Source, Argonne National Laboratory) is here only briefly introduced limiting the description to the experimental geometry and to the data reduction method. The last section (section 5.7 presents the apparatus built for the so-called quasi instantaneous thermal treatments with which all the annealed samples studied in this work were prepared. 


\subsection{SQUID Magnetometer}

The SQUID magnetometer is probably the most sensible and best known device for the characterization of a magnetic material and takes its name from one of the core components of this measurement system: the superconducting quantum interference device, which represents together with the second-derivative-coil (see below) a reliable solution for the detection of the magnetic moment of a sample, from which magnetization and magnetic susceptibility can be determined.

The main elements of the Quantum Design MPMS-5s magnetometer which was used for the magnetic measurements of all the $\mathrm{Nd}_{60} \mathrm{Fe}_{30} \mathrm{Al}_{10}$ samples are: a temperature control system, which controls the sample temperature in the range 1.8 to $400 \mathrm{~K}$ by active regulation in the specimen space of heat flow and of the helium gas flow which provides the cooling power; a magnet control system, which consists of a current power supply and of a superconducting solenoid which is able to provide stable magnetic fields in the range -4 to $+4 \mathrm{MA} / \mathrm{m}( \pm 50 \mathrm{kOe})$ operating in either persistent and non-persistent modes; a SQUID amplifier system, which constitutes the magnetic moment detection system and is based on a radio frequency SQUID detector (RF-SQUID) and highly balanced second-derivative sample coil array (see figure 5.4); a sample handling system, which gives the possibility to step (and rotate) the sample through the detection coil without inducing mechanical or electronic noise to the detection system; and, finally, a computer operating system, which controls fully automated and user-friendly all the measurement operations [119].

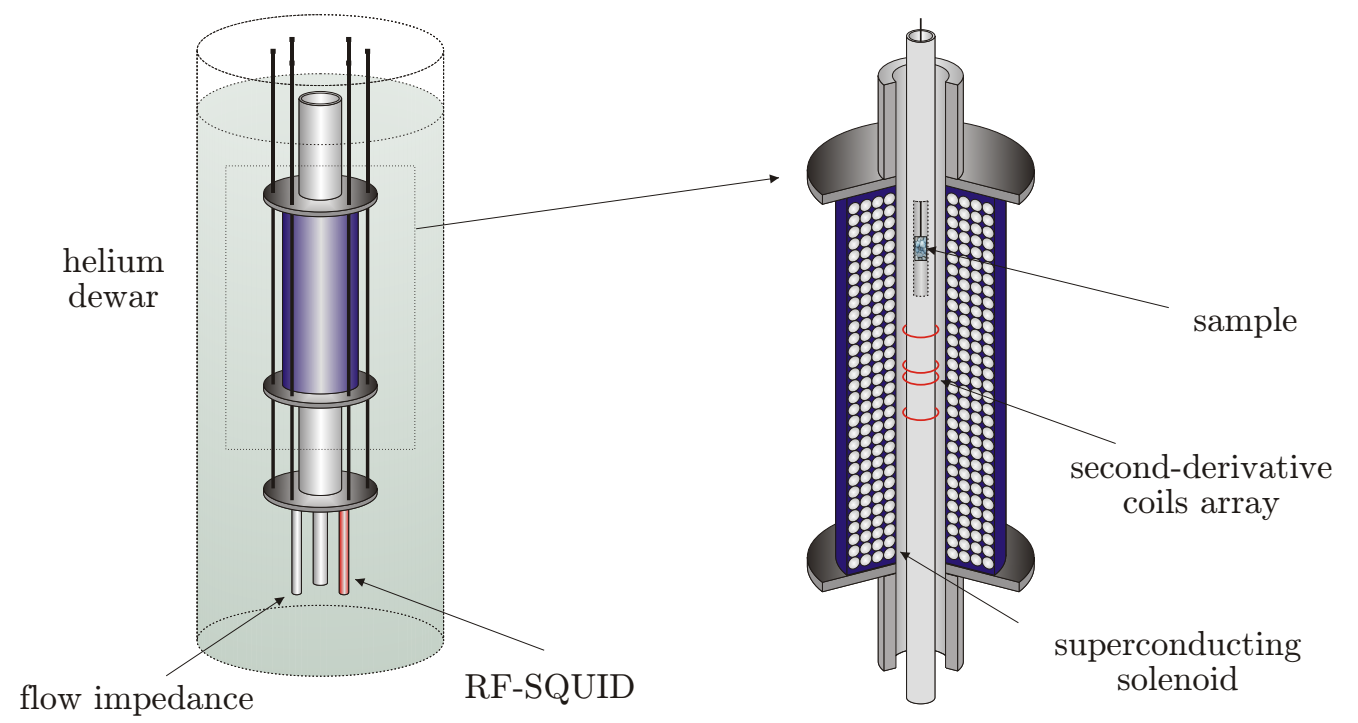

Figure 5.1. Configuration and the relative location in a helium dewar of the core elements of a SQUID MPMS-5s magnetometer.

A sketch of the core elements of a MPMS-5s magnetometer is presented in figure 5.1 showing the configuration and the relative location in a helium dewar of the supercon- 
ducting solenoid, of the detection coils, and of the sample. Helium in the liquid state $(T \leq 4.2 \mathrm{~K})$ is the unique means with which the superconducting state in several elements of a SQUID magnetometer is maintained and the cooling of the sample space is achieved.

The most important components of a SQUID magnetometer make use of the superconducting state: in the superconducting solenoid a large amount of electrical current can flow without dissipating energy and, therefore, produce a high magnetic field; in the SQUID sensor, which is basically a superconducting ring including one or two Josephson junctions, unique quantum electrodynamic phenomena (as the quantization of the magnetic flux and tunnelling effects in Josephson junctions) can take place and, therefore, be reliably used for the detection of external magnetic field $\mu_{0} H$ of the order of $10^{-15} \mathrm{~T}$.

Combining quantum mechanics and superconductivity theories, one can demonstrate that the magnetic flux $\Phi$ generated by a current flowing in a superconducting loop is quantized in units of flux quantum $\Phi_{\left[{ }^{(1)}\right.}$

$$
\Phi=n \cdot \Phi_{0}
$$

where $n=1,2,3, \ldots$ and $\Phi_{0}$ is given by the relation

$$
\begin{aligned}
\Phi_{0}=\frac{h}{2 e} & =2.067 \cdot 10^{-15} \mathrm{~V} \cdot \mathrm{s} \\
& =2.067 \cdot 10^{-15} \mathrm{~T} \cdot \mathrm{m}^{2}
\end{aligned}
$$

A simple ring cannot however be employed as active element because no flux can enter it when it is already in the superconducting state (Meissner effect). For this reason, to quantitatively use the magnetic flux quantization, one (or two) thin insulating film must be introduced in the ring to build a junction superconductor/isolator/superconductor (see figure 5.2, a so-called Josephson junction. If the junction is too thick, no superconducting current can flow, then the flux in the ring will be exactly that which is expected from the applied field because the system junction-ring behaves as a normal conductor. But if the junction is thinner than typically $30 \AA$, this effect becomes weak and the value of the supercurrent flowing in the ring is reduced by less than $10^{-5} \mathrm{~A}$. In this case the Josephson junction behaves like a tunnel-contact and a tunnelling current $I$ can flow through it. If $I<I_{c}$, where $I_{c}$ is a critical value which depends on the superconducting material and on the external magnetic field, the tunneling current is a direct current (DC) of Cooper pairs which instead break away for $I>I_{c}$ and single electrons become responsible for an alternating (AC) tunneling current.

\footnotetext{
(1) Experimentally the quantization of the magnetic flux can be proved by cooling a superconducting ring down to its transition temperature $T_{c}$ in the presence of an external magnetic field $B$. In this way a superconducting current can be frozen in the ring. On the other hand, a stationary current produces a magnetic flux $\Phi$ which can be easily measured after removing the field $B$. Repeating this experiment for different values of $B$, equation 5.1 and 5.2 can be proved.
} 


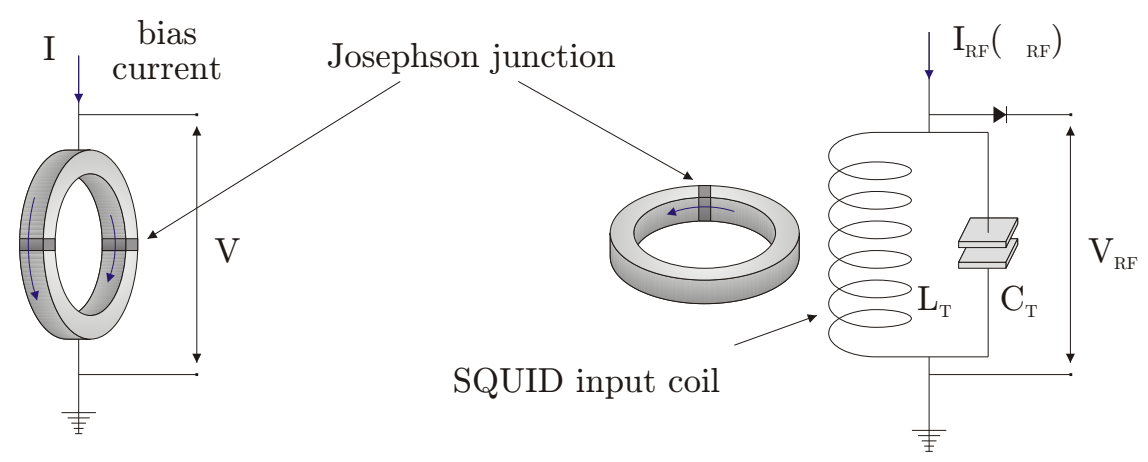

DC-SQUID

RF-SQUID

Figure 5.2. Schematic drawing of a direct current (DC-) SQUID characterized by two Josephson junctions and of a radio frequency ( $\mathrm{RF}-$ ) SQUID together with its resonant circuit.

In this configuration any magnetic flux can enter the ring due to the Josephson junction and, while the supercurrent tries to oppose it without however entirely achieving it due to the limitations of the junction itself, the current becomes a periodic function of the flux concatenated to the superconducting ring.

The relation between the flux in the ring $(\Phi)$ and the flux due to the applied field $\left(\Phi_{a}\right)$ can be expressed as:

$$
\Phi=\Phi_{a}+L_{s} \cdot I_{s}
$$

where $L_{s}$ is the inductance of the ring and $I_{s}$ is the supercurrent which produces a flux $\Phi_{s}=L_{s} \cdot I_{s}$. In the Josephson junction the supercurrent $I_{s}$ is related to the critical current $I_{c}$ by the relation:

$$
I_{s}=I_{c} \cdot \sin \left(\varphi_{2}-\varphi_{1}\right)
$$

where $\left(\varphi_{2}-\varphi_{1}\right)$ is the phase difference of the electron wavefunctions across the junction. Therefore:

$$
\Phi=\Phi_{a}+L_{s} \cdot I_{c} \sin \left(\varphi_{2}-\varphi_{1}\right)
$$

As previously shown, the flux can be expressed by equation 5.1 while the angle $\left(\varphi_{2}-\varphi_{1}\right)$ depends on the ring as expressed by the relation:

$$
\left(\varphi_{2}-\varphi_{1}\right)=2 \pi\left(n-\frac{\Phi}{\Phi_{0}}\right)
$$

from which it derives

$$
\sin \left(\varphi_{2}-\varphi_{1}\right)=-\sin \left(2 \pi \frac{\Phi}{\Phi_{0}}\right)
$$

and, therefore:

$$
\Phi=\Phi_{a}-L_{s} \cdot I_{c} \sin \left(2 \pi \frac{\Phi}{\Phi_{0}}\right)
$$


The relation between $\Phi$ and $\Phi_{a}$ is presented graphically in figure 5.3 which shows how the SQUID counts flux quanta of an external field in unit of $\Phi_{0}$. In this way it may be possible to measure any flux change $\Delta \Phi_{a}\left(>\Phi_{0}\right)$ by measuring the voltage variation $V(\Phi)$ at the ends of the Josephson junction (see figure 5.2).

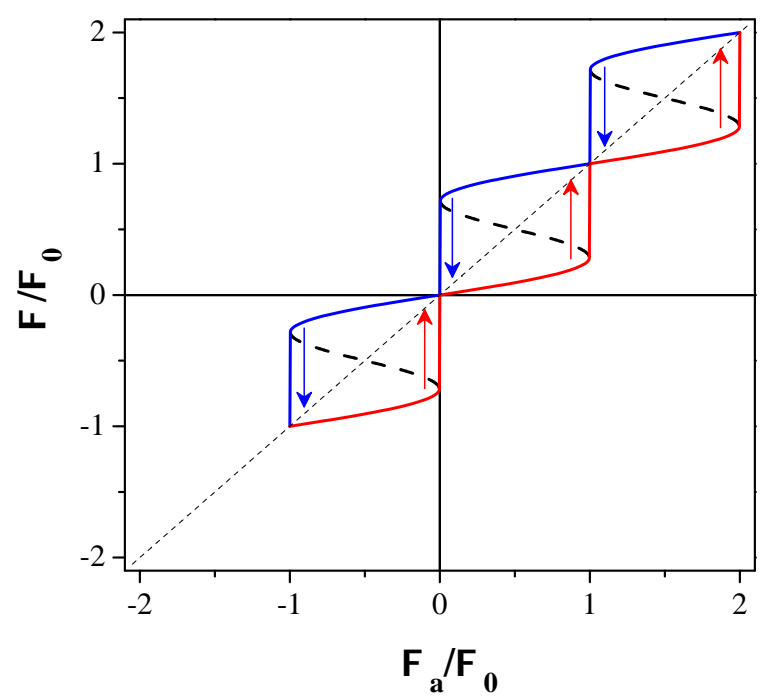

Figure 5.3. Relation deriving from the Josephson effect in a SQUID sensor between the ring flux $\Phi$ and the flux $\Phi_{a}$ due to an increasing (-) and decreasing (-) external field (both fluxes are normalized to the flux quantum $\left.\Phi_{0}\right)$.

Although its sensitivity, the SQUID in the MPMS-5s does not detect directly the magnetic dipole field generated by the specimen. Instead the sample moves through a system of superconducting detection coils as shown in figure 5.4 which are called secondderivative coils. The detection unit is a single piece of superconducting wire wound in a set of three coils configured as a second-order (second-derivative) gradiometer: the upper and the bottom coils are a single turn wound clockwise while the center coil comprises two turn wound counter-clockwise. With this configuration the noise in the detection circuit caused by fluctuations in the background and in the external magnetic field is strongly reduced while the magnetic moment of the sample can still be measured because the gradiometer measures changes in magnetic flux densities which affect locally only one of the three coils. The detection set is then connected with superconducting wires to a radio frequency (RF) SQUID which consists of a resonant unit inductively coupled to a traditional SQUID sensor. Thus, in contrast to a normal sensor (DC-SQUID), a RF-SQUID has no direct contact to any electronic components (see figure 5.2). The resonant circuit produces an alternating current $(\nu=20 / 30 \mathrm{MHz})$ which induces a variable magnetic flux and, due to the presence of the Josephson junction, determines an alternating current $I_{\mathrm{AC}}$ in the

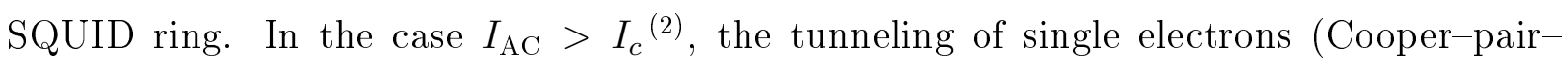

${ }^{(2)}$ That happens if the sum of the supercurrent from the second-derivative coils and the current of the 


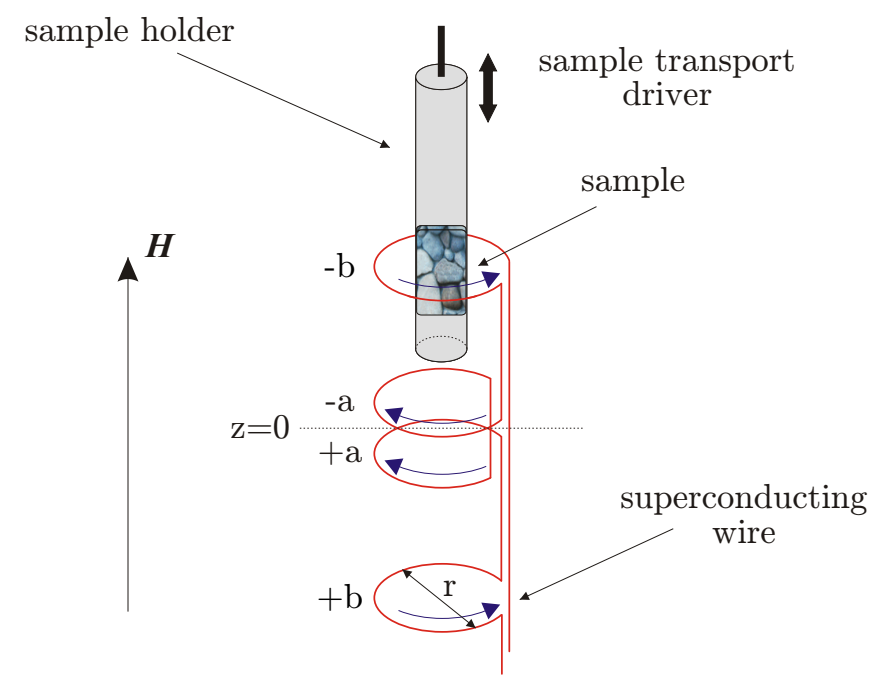

Figure 5.4. The configuration of the second-order gradiometer superconducting detection coils. The coil-set is situated outside the sample space within the liquid helium bath and in the center of the superconducting magnet.

electrons) in the junction dissipates energy as in an ohmic contact and causes a reduction of the voltage $V_{\mathrm{RF}}$ in the coupled resonant circuit which can be measured quantitatively thanks to a second semiconductor-based electronic unit. Therefore the SQUID electronic produces an output voltage proportional to the current flowing in the SQUID input coil which can be reliably filtered taking also advantage of a lock-in technique. Essentially, it can be concluded that a SQUID works as an extremely sensitive moment-current-voltage converter.

Measurements with the MPMS-5s are performed therefore by moving a sample through the superconducting detection coils, the dipole field of the magnetic specimen induces an electric current which flows persistently in the detection circuit and which is then converted to a voltage output from the SQUID detector. The absolute value of the magnetic moment can be then found calibrating accurately the system with a small piece of material having a known mass and magnetic moment, and measuring its SQUID output voltage.

An example of such output signal is shown in figure 5.5. The unknown sample moment $m$ can be then calculated by fitting the output curve by the following equation:

$$
\begin{aligned}
V(z)= & -\frac{r^{2} m}{2} \cdot\left(\frac{1}{\left[(z+b)^{2}+r^{2}\right]^{3 / 2}}-\frac{1}{\left[(z+a)^{2}+r^{2}\right]^{3 / 2}}+\right. \\
& \left.-\frac{1}{\left[(z-a)^{2}+r^{2}\right]^{3 / 2}}+\frac{1}{\left[(z-b)^{2}+r^{2}\right]^{3 / 2}}\right)
\end{aligned}
$$

resonant circuit is high enough to induce a flux variation $\Phi_{0}$ in the SQUID ring. 


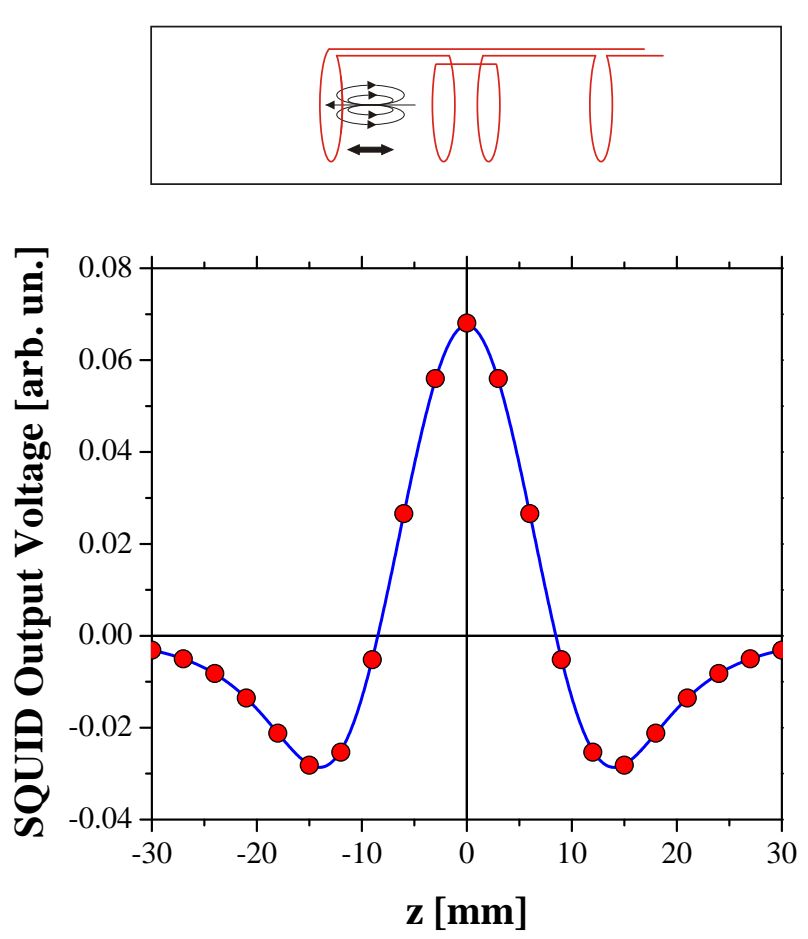

Figure 5.5. SQUID output voltage profile deriving from the movement of a sample in the second-derivative detection coils. The measurement points $(0)$ and the relative fitting $(-)$ used to calculate the magnetic moment of the specimen are indicated.

which describes the variation of $V$ as function of the sample position $z$ in a second-order gradiometer with geometrical parameters $a, b$, and $r$ (see figure 5.4).

\subsubsection{AC Susceptibility}

The MPMS-5s magnetometer is not only suitable for the determination of the equilibrium value of the magnetic moment $m(H)$ of a sample under a constant magnetic field $H(D C$ magnetometry) but also for the measurement of its $m_{A C}$ moment when an external AC field is applied (AC magnetometry). In AC magnetic measurements, a small AC driving magnetic field is superimposed on the DC field, causing a time-dependent magnetic response in the sample which can be measured by the SQUID detection coils without sample motion 117.

In order to understand what kind of information AC magnetometry can deliver, we consider firstly the case of very low frequency AC fields, where the measurement is quite similar to DC magnetometry. In this case, the magnetic moment of the sample follows the $m(H)$ curve that would be measured in a DC experiment and, as long as the AC field is small, the induced $\mathrm{AC}$ moment is

$$
m_{A C}=\frac{d m}{d H} \cdot H_{A C} \sin (2 \pi \nu t)
$$


where $H_{A C}$ is the amplitude of the driving field, $\nu$ is the driving frequency, and $\chi=$ $d m / d H$ is the slope of the $m(H)$ curve and is called magnetic susceptibility. As the DC applied magnetic field is changed, different parts of the $m(H)$ curve are accessed, giving a different $\chi$. Since the AC measurement is sensitive to the slope of $m(H)$ and not to its absolute value, small magnetic changes can be easily detected. At higher frequencies, the AC moment of the sample does not follow the DC magnetization curve anymore due to dynamic effects in the sample. For this reason, the AC susceptibility is often known as the dynamic susceptibility because the magnetization of the sample may lag behind the drive field and the SQUID circuitry can detect this effect yielding two quantities: the magnitude of the susceptibility $(\chi)$ and the phase shift $(\phi)$ relative to the drive signal. Similarly, one can express the susceptibility by an in-phase (or real) component $\chi^{\prime}$ and an out-of-phase (or imaginary) component $\chi^{\prime \prime}$. The two representations can be related to each other by the expressions:

$$
\begin{aligned}
\chi^{\prime} & =\chi \cos (\phi) \\
\chi^{\prime \prime} & =\chi \sin (\phi)
\end{aligned}
$$

or, alternatively:

$$
\begin{aligned}
& \chi=\sqrt{\left(\chi^{\prime}\right)^{2}+\left(\chi^{\prime \prime}\right)^{2}} \\
& \phi=\arctan \left(\frac{\chi^{\prime \prime}}{\chi^{\prime}}\right)
\end{aligned}
$$

In the limit of low frequencies where an AC measurement is most similar to a DC measurement, the real component $\chi^{\prime}$ is just the slope of the $m(H)$ curve while the imaginary component $\chi^{\prime \prime}$ indicates in general dissipative processes in the sample (as, for example, by eddy currents in conductive samples). A nonzero $\chi^{\prime \prime}$ is observed in relaxation and irreversibility processes in spin-glasses, in irreversible domain wall movement or absorption due to a permanent moment in ferromagnets, and is often used to measure transition temperatures (both $\chi^{\prime}$ and $\chi^{\prime \prime}$ are very sensitive to thermodynamic phase changes).

\subsection{Vibrating Sample Magnetometer (VSM)}

The VSM magnetometer offers possibilities for the magnetic characterization of a sample which cannot yet be offered by any other magnetometer (torque magnetometer, force balance, magnetic force microscopy, etc.): sample size, in-plane and out-plane magnetization measurements, sample temperatures up to $1200 \mathrm{~K}$. For this reasons, an Oxford Instruments VSM-5 magnetometer was used together with the SQUID magnetometer for the characterization of the $\mathrm{Nd}_{60} \mathrm{Fe}_{30} \mathrm{Al}_{10}$ specimens.

The name VSM magnetometer derives from the solution used to measure reliably the moment of a magnetic sample by mean of magnetic induction effects. In a VSM, as well as for the previously described SQUID, any variation of the magnetic dipole field of a 
specimen induces a voltage/current in a detection coil which outputs a signal proportional to the sample magnetic moment.

In the case of the VSM, this signal is an e.m.f. equal to the rate of change of flux linking the circuit

$$
V=-N \frac{d \Phi}{d t}
$$

where $N$ is the number of turns of the coil. From the knowledge of the coil cross-sectional area $A$, the magnetic induction $B$ can be calculated as $B=\Phi / A$ and $V$ can be expressed as

$$
V=-N A \frac{d B}{d t}
$$

If the sample moves periodically with a known frequency $\nu$, the flux variation concatenated with the coil becomes a function of $\nu$ and the magnetization $M$ of a sample is detected by the VSM as a periodic function:

$$
M^{\prime}=c \cdot M \sin (2 \pi \nu t)
$$

where $c$ is a constant which depends on the geometrical shape of the sample.

The magnetic induction can then be rewritten as:

$$
B(t)=\mu_{0}\left(H+M^{\prime}\right)
$$

Therefore, the change in induction is then simply:

$$
\frac{d B}{d t}=2 \pi \nu c \mu_{0} M \cos (2 \pi \nu t)
$$

and

$$
V=-2 \pi \nu N A c \mu_{0} M \cos (2 \pi \nu t)
$$

From this relation the magnetization $M$ can be then easily calculated after a calibration of the magnetometer with a piece of material of known mass and magnetic moment which permits to determine the value of the constant $c$.

A schematic drawing of a VSM magnetometer is presented in figure 5.6. The detection system is constituted by two identical pairs of normal-conducting copper coils and a periodic variation of the magnetic dipole field is achieved by moving periodically (with a frequency of $55 \mathrm{~Hz}$ and an amplitude of $1.5 \mathrm{~mm}$ ) the sample through them (3). The main advantage of the periodic output signal is the possibility to filter out any component with frequency different from $55 \mathrm{~Hz}$ (lock-in modus) and to measure only the induction due to the sample movement. A second detail which further reduces most of the noise arising from the superconducting magnet and from the background derives from the fact that the two coil pair are connected in parallel with the upper pair wound clockwise and the

\footnotetext{
${ }^{(3)}$ For this reason the sample and its holder vibrates on their vertical axis in the middle of the detection coils and of the superconducting split-coils which are used to produce the magnetic field.
} 


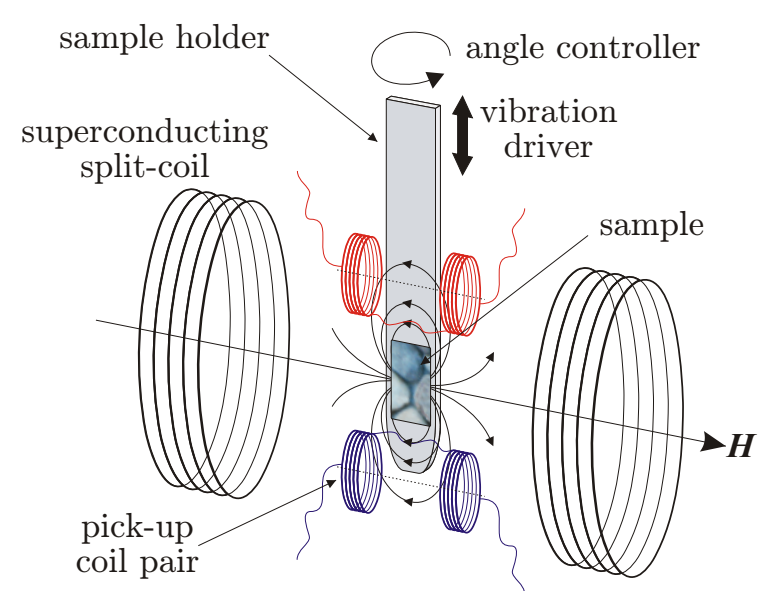

Figure 5.6. Schematic drawing of a vibrating sample magnetometer (VSM) which shows the superconducting split-coils, the detection coils (pick-up coils) and the relative position of the sample.

bottom one counterclockwise. In this way, the signal from the dipole field of the sample is always doubled while any signal which is uniform within the size of the detection set-up $(\approx 40 \mathrm{~mm})$ is averaged out.

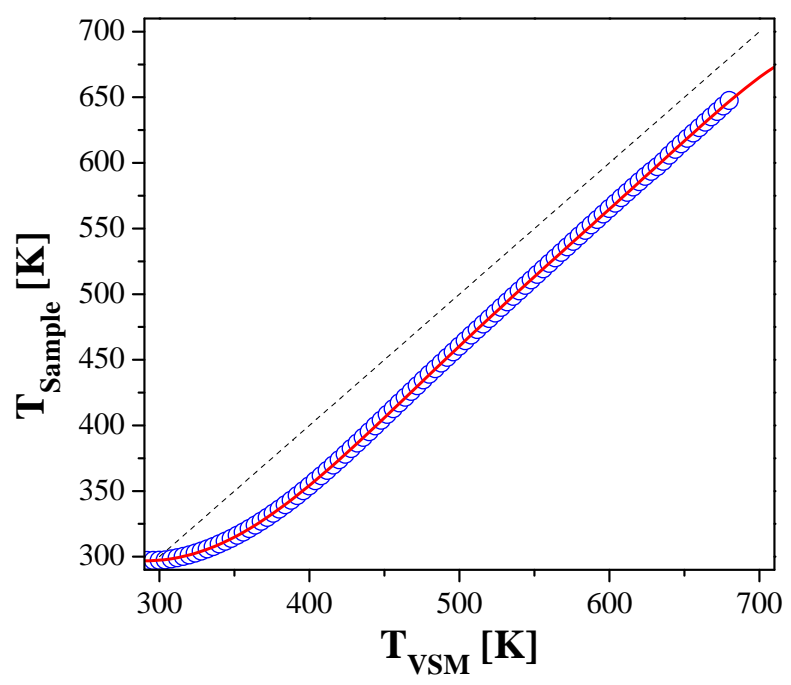

Figure 5.7. Effective sample temperature ( $\left.T_{\text {sample }}\right)$ as function of the temperature indicated by the temperature control unit ( $\left.T_{\mathrm{VSM}}\right)$ in a high-temperature vibrating sample magnetometer.

The VSM magnetometer was mainly used for measurements of the hysteresis loops in the sample plane (the so-called in-plane measurements), perpendicular to it (outplane), for thermomagnetic scans in the range $[300,650] \mathrm{K}$, and for measuring hysteresis loops at high temperatures $([400,650] \mathrm{K})$. In the latter two cases, a large difference between the effective sample temperature $\left(T_{\text {sample }}\right)$ and the temperature indicated by the 
$\operatorname{VSM}\left(T_{\mathrm{VSM}}\right) \sqrt{(4)}$, was measured and a correction had to be introduced. In the case of temperature scans, this correction can be expressed by the relation:

$$
T_{\text {sample }}=a_{0}+\sum_{n=1}^{6} a_{n} \cdot\left(T_{\mathrm{VSM}}\right)^{n}
$$

where $a_{0}, \ldots, a_{6}$ (see table 5.1) are coefficients determined by fitting the curve $T_{\text {sample }}$ vs. $T_{\mathrm{VSM}}$ (see figure 5.7) which was measured by using of a thermocouple as sample. Corrections to the sample temperature $T_{\text {sample }}$ were also found to be necessary in the

\begin{tabular}{cccc}
\hline Coefficient & Value & Coefficient & Value \\
\hline$a_{0}$ & -1593.41 & $a_{4}$ & $-1.13^{-6}$ \\
$a_{1}$ & 32.04 & $a_{5}$ & $9.78^{-10}$ \\
$a_{2}$ & -0.208 & $a_{6}$ & $-3.40^{-13}$ \\
$a_{3}$ & $6.71^{-4}$ & & \\
\hline
\end{tabular}

Table 5.1. Coefficients of the polynomial curve expressed by equation 5.19 .

case of isothermal experiments (after a transient time $t_{\text {relax }}$ during which $T_{\text {sample }}$ relaxes exponentially toward $\left.T_{\mathrm{VSM}}\right)$. In this case $T_{\text {sample }}$ and the relative $T_{\mathrm{VSM}}$ are listed for clarity in table 5.2 .

\begin{tabular}{cccccc}
\hline$T_{\text {sample }}[\mathrm{K}]$ & $T_{\text {VSM }}[\mathrm{K}]$ & $t_{\text {relax }}[\mathrm{s}]$ & $T_{\text {sample }}[\mathrm{K}]$ & $T_{\text {VSM }}[\mathrm{K}]$ & $t_{\text {relax }}[\mathrm{s}]$ \\
\hline 300 & 303.0 & 1800 & 440 & 462.3 & 767 \\
320 & 333.4 & 1190 & 460 & 482.9 & 519 \\
340 & 356.3 & 1351 & 480 & 503.6 & 511 \\
360 & 379.1 & 1243 & 500 & 524.3 & 373 \\
380 & 400.0 & 1204 & 520 & 544.5 & 340 \\
400 & 420.9 & 1023 & 540 & 564.5 & 295 \\
410 & 431.3 & 899 & 560 & 584.6 & 215 \\
420 & 441.6 & 900 & 580 & 604.8 & 246 \\
430 & 452.0 & 856 & 600 & 625.1 & 172 \\
\hline
\end{tabular}

Table 5.2. Sample temperature as indicated by the VSM ( $\left.T_{\mathrm{VSM}}\right)$, effective sample temperature $\left(T_{\text {sample }}\right)$, and relative transient time $\left(t_{\text {relax }}\right)$ as experimentally determined in a high-temperature vibrating sample magnetometer.

\footnotetext{
${ }^{(4)} T_{\mathrm{VSM}}$ is the temperature indicated by the VSM as the temperature of the sample space close to the heater and it is usually considered as the temperature of the sample. However, as evident from figure 5.7 differences between $T_{\text {sample }}$ and $T_{\mathrm{VSM}}$ even larger than $50 \mathrm{~K}$ were measured.
} 


\subsection{Small Angle Neutron Scattering}

Small angle neutron scattering (SANS) is one of the techniques that go under the collective name of small angle scattering (SAS) in which radiation (neutrons, $\mathrm{x}$-rays or light) is scattered by a sample and the resulting scattering pattern is analyzed for small scattering vectors to provide information about size, shape and orientation of some of its components. The type of sample that can be studied, the sample environment that can be used, the actual length scales that can be probed and the information that can ultimately be obtained by the analysis of the scattering patterns depend on the nature of the radiation in use and, hence, on the kind of interaction between radiation and sample 175 .

The main advantages of SANS with respect to $\mathrm{x}$-rays or light SAS are the length scales which neutrons cover $(1-100 \mathrm{~nm})$ and the weakness of the neutron-matter interaction which permits to obtain undistorted information at an atomic level on the chemical and physical properties of the specimens. This aspect is of fundamental importance also from the theoretical point of view since the interpretation of neutron scattering experiments can concern in good approximation only with the properties of the target sample, with no distortion by the neutron probe. Thus, the information provided by neutron scattering are obtainable in no other way.

To cover the different experimental and theoretical aspects of small angle neutron scattering, the properties of neutron radiation, the nature of neutron-matter interaction, and the different types of beamlines used during this work are described separately in following sections (5.3.1 5.3.6). The description of the theoretical models and of the relative expressions of the small angle cross-section, which will be utilized in the analysis of the experimental scattering data, are instead presented in appendix B.

\subsubsection{Neutron Radiation}

The use of neutrons as a probe in the study of condensed matter derives mainly from their intrinsic properties (see table 5.3): mass, spin, electric charge, etc.

The energy $E$ of a neutron with mass $m_{n}$ and wave vector $\boldsymbol{k}$ is given by the relation (considering the de Broglie/Schrödinger wave-particle dualism principle)

$$
E=\frac{\hbar^{2} k^{2}}{2 m_{n}}
$$

thus, a neutron with a wave vector $k$ of $1.5 \AA^{-1}$ (wavelength $\lambda=4.19 \AA$ ) possesses an energy $E=4.7 \mathrm{meV}$ which is, by contrast, more than 300.000 times smaller than the energy of a $4.19 \AA \mathrm{x}$-ray photon $(1.5 \mathrm{keV})$. Hence, the properties of the target sample in a neutron scattering experiment are only weakly distorted by the neutron probe if compared to $\mathrm{x}$-ray scattering since the energy of a single neutron is well below the typical ionization energies, activation energies for diffusion or for any other chemical reaction. A 


\begin{tabular}{ccc}
\hline Properties & Symbol & Value \\
\hline Mass & $m_{n}$ & $1.6751 \times 10^{-27} \mathrm{~kg}$ \\
Spin & $S_{n}$ & $\frac{1}{2} \hbar$ \\
Electric charge & $q_{n}$ & 0 \\
Magnetic moment & $\mu_{n}$ & $9.6626 \times 10^{-27} \mathrm{~J} / \mathrm{T}$ \\
Electric dipole moment & $P_{n}$ & 0 \\
Half-life & $\tau_{1 / 2}$ & $888 \mathrm{~s}$ \\
\hline
\end{tabular}

Table 5.3. Physical properties of neutrons. (For comparison: $m_{n}=1839 \cdot m_{e}, S_{n}=S_{e}$, $\mu_{n}=1 / 961 \cdot \mu_{e}$, where $m_{e}, S_{e}$ and $\mu_{e}$ are the mass, the spin and the magnetic moment of an electron, respectively).

second fundamental difference between neutrons and electromagnetic radiation emerges considering the mechanism by which the incident radiation interacts with matter: light and $\mathrm{x}$-rays are in fact both scattered by electrons which surrounds atomic nuclei while neutrons are scattered by the nucleus itself (nuclear scattering).

Those differences and the intrinsic properties of neutron have several other important consequences:

- In the case of electromagnetic radiation, the scattering cross-section of an atom increases with $Z^{2}$ being $Z$ the atomic number, while in the case of neutrons the strength of the neutron-nucleus interaction varies completely irregularly with $Z$ (see table 5.4) and not even isotopes of the same element have the same neutron scattering cross-section (e.g., hydrogen and deuterium).

\begin{tabular}{cccc}
\hline Atom & $\sigma_{\text {coh }}[$ barn] & Atom & $\sigma_{\text {coh }}[$ barn] \\
\hline${ }^{1} \mathrm{H}$ & 1.758 & $\mathrm{Ga}$ & 6.675 \\
${ }^{2} \mathrm{D}$ & 5.592 & $\mathrm{~N}$ & 11.010 \\
$\mathrm{Al}$ & 1.496 & $\mathrm{O}$ & 4.232 \\
$\mathrm{Fe}$ & 11.220 & $\mathrm{Mn}$ & 1.750 \\
$\mathrm{Nd}$ & 7.430 & & \\
\hline
\end{tabular}

Table 5.4. Neutron scattering coherent cross-sections of some elements and isotopes. $\left(1\right.$ barn $\left.=1 \times 10^{-28} \mathrm{~m}^{2}\right)$.

- Atomic nuclei are about $10^{4}-10^{6}$ times smaller than typical neutron wavelengths, hence the nuclei effectively act as point scatterers and, consequently, the nuclear scattering remains constant as the scattering angle increases and the scattering is spherically symmetric. This is in contrast to the case of $\mathrm{x}$-rays where atomic 
diameters are only $0.1-10$ times the typical neutrons wavelengths, resulting in a decrease in the scattered intensity with increasing angle.

- The interaction of neutrons with matter is weak and the absorbtion of neutrons by most materials is correspondingly small. Neutron radiation is therefore very penetrating and can be used to probe the bulk properties of samples with thickness of several millimeters (or of samples contained inside complex ancillary equipments as cryostats, furnaces, pressure cells, shear apparatus, etc). On the contrary, $\mathrm{x}$-rays require energies of about $10^{5} \mathrm{eV}$ to penetrate a $1 \mathrm{~mm}$ thick sample (see section 5.6 ).

- The neutron has a small magnetic moment. This can interact with the spin and the orbital magnetic moments of atoms with unpaired electrons, giving rise to an additional scattering contribution of magnetic origin (magnetic scattering).

The neutron wavelength and, consequently, the length scales covered in a scattering experiment depend through equation 5.20 on the neutron energy, which must therefore be selected on the basis of the desired experimental conditions. For this reason neutrons beamlines are often called as being cold, thermal, hot, or epithermal depending on the neutrons that they employ. There is no standard definition of these terms, although there is a consensus that thermal neutrons have an energy of $25 \mathrm{meV} \approx 300 \mathrm{~K}\left(T_{\text {neutron }}=E / k_{B}\right)$. An accepted definition of the thermal nomenclature for neutron energies is reported in table 5.5 .

\begin{tabular}{ccc}
\hline Neutrons & $E[\mathrm{meV}]$ & $\lambda[\AA]$ \\
\hline Cold & $\leq 10$ & $\geq 3$ \\
Thermal & $10-100$ & $1-3$ \\
Hot & $100-500$ & $0.4-1$ \\
Epithermal & $\geq 500$ & $\leq 0.4$ \\
\hline
\end{tabular}

Table 5.5. Neutrons nomenclature as function of neutron energy and wavelength.

The beamlines utilized during this work (see section 5.3.6) make use of cold neutrons whose wavelengths range from some $\AA$ up to $15 \AA$.

\subsubsection{Nuclear Scattering}

In a small angle scattering experiment, a beam of collimated neutrons interacts with a small volume $V$ of a sample $\left(V=A \cdot t_{s}\right.$, where $A$ is the cross-sectional area of the beam and $t_{s}$ is the thickness of the sample). Some of the incident radiation is transmitted by the sample, some is absorbed and some is scattered ${ }^{(5)}$. A detector (or detector element)

${ }^{(5)}$ Only elastic neutron scattering will be here considered. 

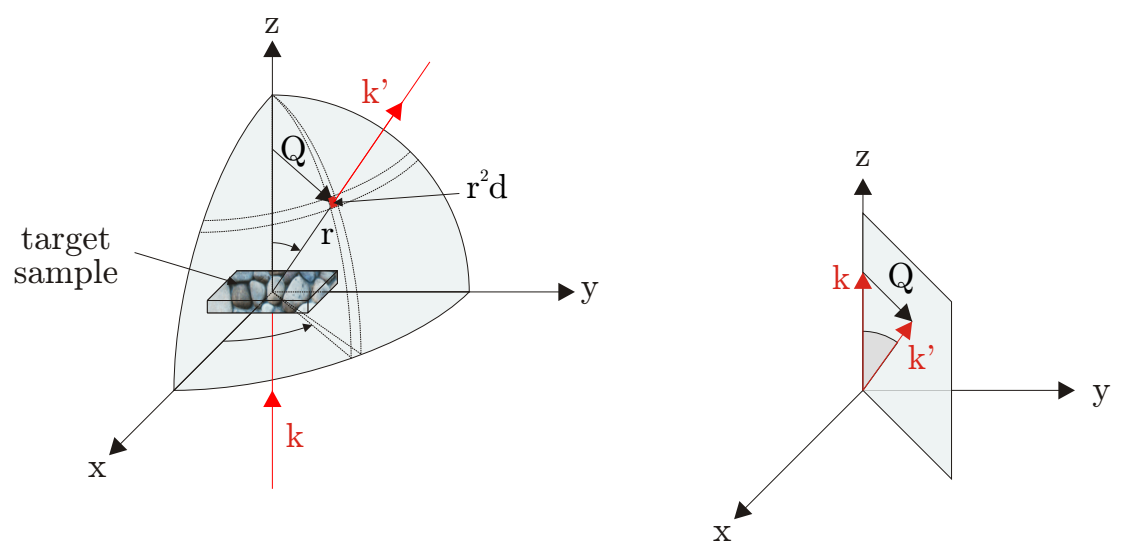

Figure 5.8. Schematic geometry of a small angle scattering event. $k$ and $k^{\prime}$ are the incident and the scattered wavevectors, respectively, and $Q$ is the resultant scattering vector. $r^{2} d \Omega$ is the cross section area.

of dimensions $\Delta x \times \Delta y$ positioned at some distance $L_{s d}$ and scattering angles $\theta$ and $\phi$ from the sample (see figure 5.8 records then the flux $I(\lambda, \theta, \phi)$ of radiation scattered into a solid angle element $\Delta \Omega . I(\lambda, \theta, \phi)$ may be expressed in general terms as

$$
I(\lambda, \theta, \phi)=I(Q)=I_{0}(\lambda) \zeta(\lambda) \Delta \Omega T V \cdot \frac{d \sigma}{d \Omega}(Q)
$$

where $I_{0}$ is the incident flux, $\zeta$ is the detector efficiency, $T$ is the sample transmission, $d \sigma / d \Omega(Q)$ is the microscopic differential cross-section [110], and $Q$ is the scattering vector which is defined as

$$
Q=|\boldsymbol{Q}|=\left|\boldsymbol{k}^{\prime}-\boldsymbol{k}\right|=\frac{4 \pi}{\lambda} \sin \left(\frac{\phi}{2}\right)
$$

where $\boldsymbol{k}$ and $\boldsymbol{k}^{\prime}$ are the incident and scattered wavevectors respectively, $\lambda$ is the neutron wavelength and $\phi$ is the scattering angle (see figure 5.8).

The first three terms of equation 5.21 are clearly instrument-specific while the last three terms are sample-dependent. The aim of a SANS experiment is therefore to determine the differential cross-section which contains all the information on the shape and size of the scattering bodies in a sample and on the nature of their interaction with neutrons. The differential cross section in a SANS experiment is expressed as the relative number of neutrons which are scattered from the examined specimen in a defined direction $Q$ or, similarly, as the ratio

$$
\frac{d \sigma}{d \Omega}(Q)=\frac{\text { scattered neutrons inside a solid angle } d \Omega \text { per nucleus per sec }}{\text { number of incident neutrons per } \mathrm{cm}^{2} \text { per sec }}
$$

Finding an analytical expression for the cross section arising from the neutron-matter interaction is a complex problem which can be here only summarized and approximated. The first assumption is to consider the neutron-matter interaction as the sum of single scattering events between a free nucleus and a neutron. In this case, the incoming neutron 


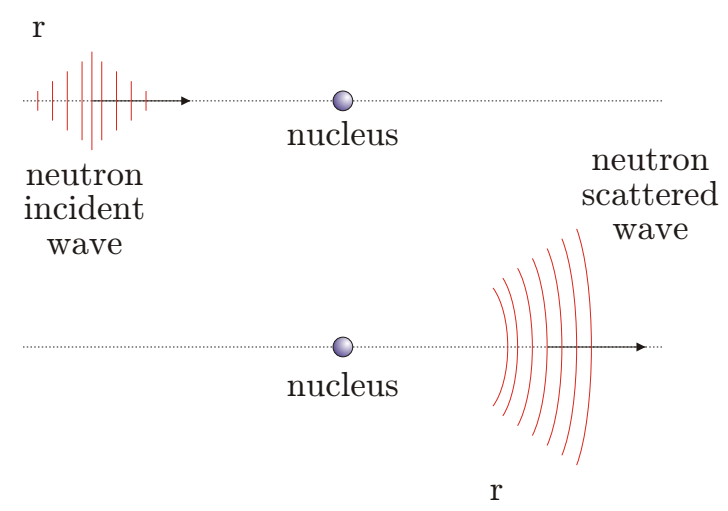

Figure 5.9. Schematic representation of the scattering process of an incident neutron on a free nucleus.

beam (described by a plane wave due to the high degree of collimation of the neutron beam) is scattered by the nucleus in a spherical wave (see figure 5.9 [28, p. 438] and the corresponding wavefunction is asymptotically a superposition of an incident plane wave and of a spherical one

$$
\lim _{\Delta \boldsymbol{r} \rightarrow+\infty} \psi(\boldsymbol{r}, t)=\exp (i \boldsymbol{k} \cdot \boldsymbol{r})+\frac{f\left(\left|\boldsymbol{k}-\boldsymbol{k}^{\prime}\right|\right)}{|\Delta \boldsymbol{r}|} \exp \left(i \boldsymbol{k}^{\prime} \cdot \Delta \boldsymbol{r}\right)
$$

where $\Delta \boldsymbol{r}=\left(\boldsymbol{r}-\boldsymbol{r}^{\prime}\right)$ and $f\left(\left|\boldsymbol{k}-\boldsymbol{k}^{\prime}\right|\right)$ is the scattering amplitude. For thermal/cold neutrons $f(Q)$ can be approximated (s-wave scattering) as

$$
f(Q)=\frac{1}{2 i Q}\left[\exp \left(i \delta_{0}\right)-1\right] \cong-a+i Q \cdot a^{2}+\ldots
$$

where $\delta_{0}$ is a "phase shift" and $a$ the so-called scattering length $[141$. But no nucleus is completely free since the nuclei in a solid are relatively bound and the according effects on scattering can be accounted for a bound scattering length by defining

$$
b=a\left(1+\frac{1}{Z}\right)
$$

where $Z$ is the atomic number of the scattering nucleus, and $f(Q)$ becomes

$$
f(Q) \cong-b+i Q \cdot b^{2}+\ldots
$$

The scattering amplitude $f(Q)$ is related to the differential scattering cross section by the relation 110

$$
\frac{d \sigma}{d \Omega}(Q) \propto\langle\psi \mid \psi\rangle \propto|f(Q)|^{2}
$$

\footnotetext{
${ }^{(6)}$ The scattering length is a parameter which depends on the scattering nucleus and which can be a complex number if absorption is not negligible: $a=a^{\prime}+i a^{\prime \prime}$. Its name derives from the dimension of the function $f(Q)$ which is that of a length.
} 
which explains why the scattering cross section contains information on the structure of the scattering nucleus and on the nature of the interaction nuclei-neutron.

In a real scattering experiment a sample is not simply a bound scattering body but a complex array of spatially distributed nuclei. Hence, in good approximation, the total scattering cross section can be expressed as sum of $N$ independent contributions

$$
\frac{d \sigma}{d \Omega}(\boldsymbol{Q})=\frac{1}{N} \sum_{j=1}^{N}\left|b_{j} \cdot \exp \left(i \boldsymbol{Q} \cdot \boldsymbol{r}_{j}\right)\right|^{2}
$$

where $b_{j}$ is the bound scattering length of the $j$-th scatterer and $\exp \left(i \boldsymbol{Q} \cdot \boldsymbol{r}_{j}\right)$ is an interference term which expresses the relative spatial distribution of the $N$ nuclei.

Replacing the sum over nuclei with an integral over a volume with constant scattering length, the previous relation becomes

$$
\frac{d \sigma}{d \Omega}(\boldsymbol{Q})=\frac{1}{N}\left|\int_{V} \eta_{n u c l}(\boldsymbol{r}) \cdot \exp (i \boldsymbol{Q} \cdot \boldsymbol{r}) d \boldsymbol{r}\right|^{2}
$$

where

$$
\eta_{\text {nucl }}=\sum_{j=1}^{N} \frac{b_{j}}{V}
$$

is the nuclear scattering length density and expresses the average scattering length of the $N$ nuclei over the their volume $V$. The neutron length densities of some elements of interest are reported for example in table 5.6 .

\begin{tabular}{ccc}
\hline Atom & $\left(b_{\text {bound }}\right)_{\text {coh }}[\mathrm{fm}]$ & $\left(\eta_{\text {nucl }}\right)_{\text {coh }} \times 10^{6}\left[\AA^{-2}\right]$ \\
\hline${ }^{1} \mathrm{H}$ & -3.741 & -2.01 \\
${ }^{2} \mathrm{D}$ & 6.671 & 1.80 \\
$\mathrm{~N}$ & 9.360 & 5.03 \\
$\mathrm{Al}$ & 3.449 & 2.08 \\
$\mathrm{Fe}$ & 9.450 & 8.02 \\
$\mathrm{Mn}$ & -3.73 & -3.04 \\
$\mathrm{Nd}$ & 7.690 & 2.18 \\
$\mathrm{Ga}$ & 7.288 & 3.72 \\
\hline
\end{tabular}

Table 5.6. Neutron scattering length densities of some elements and isotopes.

The previous formalism can be applied with only minor changes also to a macroscopically heterogeneous sample composed of two phases of different chemical compositions and, hence, with different nuclear lengths densities and volumes. The cross section becomes in this case

$$
\frac{d \sigma}{d \Omega}(\boldsymbol{Q})=\frac{1}{N}\left|\int_{V_{1}} \eta_{1} \cdot \exp (i \boldsymbol{Q} \cdot \boldsymbol{r}) d \boldsymbol{r}_{1}+\int_{V_{2}} \eta_{2} \cdot \exp (i \boldsymbol{Q} \cdot \boldsymbol{r}) d \boldsymbol{r}_{2}\right|^{2}
$$


where $V_{i}$ is the volume of the phase $i$ and $\eta_{i}$ is its mean scattering length density

$$
\eta_{i}=\sum_{j=1}^{N_{i}} \frac{c_{j} b_{j}}{\Omega_{j}}
$$

where $c_{j}$ is the concentration, $b_{j}$ the nuclear scattering length and $\Omega_{j}$ the atomic volume of the $j$-th constituent of the phase $i\left(V_{i}=\sum_{j} \Omega_{j}\right)$.

Equation 5.31 can be rewritten as

$$
\begin{aligned}
\frac{d \sigma}{d \Omega}(\boldsymbol{Q})= & \frac{1}{N} \mid \int_{V_{1}} \eta_{1} \cdot \exp (i \boldsymbol{Q} \cdot \boldsymbol{r}) d \boldsymbol{r}_{1}+ \\
& +\left.\eta_{2}\left(\int_{V} \exp (i \boldsymbol{Q} \cdot \boldsymbol{r}) d \boldsymbol{r}-\int_{V_{1}} \exp (i \boldsymbol{Q} \cdot \boldsymbol{r}) d \boldsymbol{r}_{1}\right)\right|^{2}
\end{aligned}
$$

considering that $V=V_{1}+V_{2}$ and becomes, in good approximation (7).

$$
\frac{d \sigma}{d \Omega}(\boldsymbol{Q}) \simeq \frac{1}{N}\left(\eta_{1}-\eta_{2}\right)^{2}\left|\int_{V_{1}} \eta_{1}(\boldsymbol{r}) \cdot \exp (i \boldsymbol{Q} \cdot \boldsymbol{r}) d \boldsymbol{r}_{1}\right|^{2}
$$

The scattering cross section is therefore a function of two quantities: the square of the difference in neutron scattering length density, or nuclear contrast, $\Delta \eta_{n u c l}^{2}=\left(\eta_{1}-\eta_{2}\right)^{2}$ and a sample dependent term which is called the static structure factor, or form factor, $|F(Q)|^{2}$. The contrast is simply the squared difference in $\eta_{\text {nucl }}$ between the scattering body and the surrounding medium and clearly, if $\Delta \eta_{n u c l}^{2}$ is zero, then equation 5.34 is also zero and there is no SANS. Moreover, the fact that the scattering contrast is a squared term implies that any information on which part of the sample is actually responsible for the scattering is lost

$$
\begin{aligned}
& \Delta \eta<0 \leftrightarrow \eta_{1}<\eta_{2} \\
& \Delta \eta>0 \leftrightarrow \eta_{1}>\eta_{2}
\end{aligned}
$$

and, hence, the scattering patterns arising from a two-phases systems as those in figure 5.10 are identical (apart from a different background intensity).

The form factor is basically a function that describes how $d \sigma / d \Omega(\boldsymbol{Q})$ is modulated by interference effects between radiation scattered by different parts of the same scattering body and it is therefore strongly dependent on its shape. The general form of $F(Q)$ is

${ }^{(7)}$ The term

$$
\int_{V} \exp (i \boldsymbol{Q} \cdot \boldsymbol{r}) d \boldsymbol{r}
$$

in equation 5.34 represents the structure factor of the whole sample and it can be neglected if the sample has a macroscopic size and the cross section is measured at $Q \neq 0$. 


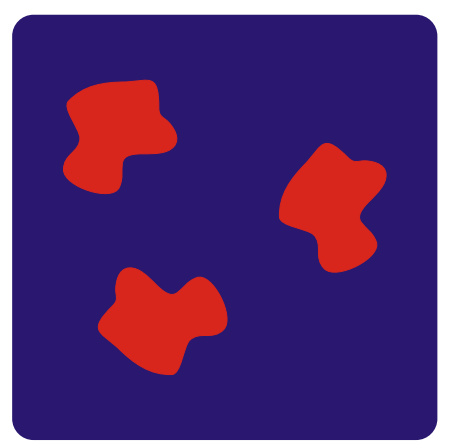

$\eta_{1}<\eta_{2}$

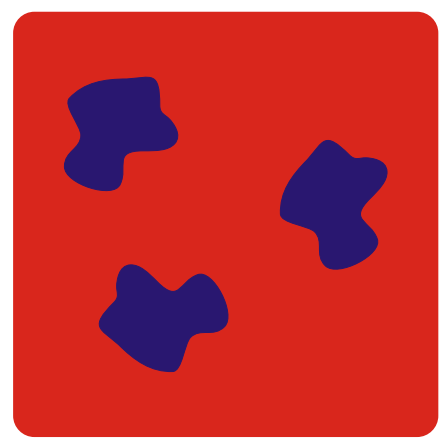

$\eta_{1}>\eta_{2}$

Figure 5.10. Example of two-phases systems with $\Delta \eta_{\text {nucl }}=\left(\eta_{1}-\eta_{2}\right)<0$ and $\Delta \eta_{\text {nucl }}>0$, respectively. The two systems give the same SANS cross section apart a different background intensity.

given by the Van de Hulst's equation 164

$$
|F(\boldsymbol{Q}, R)|^{2}=\frac{1}{V_{p}^{2}(R)}\left|\int_{V_{p}(R)} \exp [i f(\boldsymbol{Q} \cdot \boldsymbol{r})] d \boldsymbol{r}\right|^{2}
$$

where $V_{p}$ is the volume of the scattering body, $R$ is a "shape parameter" that might represent a characteristic length (for example, a radius in the case of spherical particles) or a radius of gyration, and $f$ is a function of $Q$ and $R$. Analytic expressions of $|F(\boldsymbol{Q}, R)|^{2}$ exist for most common shapes and some examples of form factors are presented in appendix B. Figure 5.11 shows, for example, the form factor of spherical scatterers of radius $R=50 \AA$ which is given by the expression

$$
|F(\boldsymbol{Q}, R)|^{2}=\frac{3}{4 \pi R^{3}}\left|\frac{\sin (Q R)-Q R \cos (Q R)}{(Q R)^{3}}\right|^{2}
$$

The positions of the maxima $Q_{i}$, in the so-called Porod's regime $(Q \cdot R>1)$, satisfy the relation

$$
Q_{n} \cdot R=n \cdot \pi \quad \text { where } n=1,2,3, \ldots
$$

and permit to measure $R$ and, indirectly, to define the resolution limits of a SANS instrument. In fact, if the scattering vector $Q$ is limited experimentally in the range $\left[Q_{\min }, Q_{\max }\right]$, then the size $d$ of a scattering body which can be detected in a SANS experiment is

$$
\frac{2 \pi}{Q_{\max }} \leq d \leq \frac{2 \pi}{Q_{\min }}
$$

and hence, for $Q \in[0.004,0.6] \AA^{-1}$ which is a typical $Q$-range in SANS experiments, only scatterers between 1 and $\sim 150 \mathrm{~nm}$ can be resolved. 


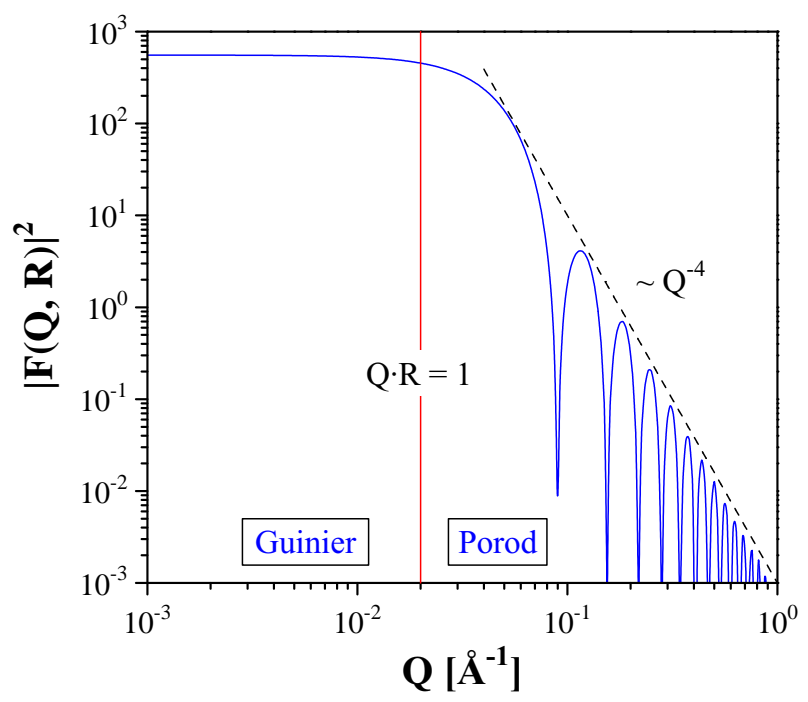

Figure 5.11. Calculated form factor $|F(\boldsymbol{Q}, R)|^{2}$ for spherical scattering bodies with radius $R=50 \AA$. The condition $Q \cdot R=1(-)$ defines the transition between the Guinier's and Porod's regimes.

Beyond the form factor, which accounts for interference between different parts of the same scattering body, an additional term must be introduced in equation 5.34, if interparticles interference between bodies with equal scattering length density is present. This term is the so-called structure factor (or, interparticle structure factor) $S(\boldsymbol{Q})$ and is commonly expressed by the relation

$$
S(\boldsymbol{Q}, \xi)=1+\frac{4 \pi N}{Q V(\xi)} \int_{0}^{+\infty}[g(\boldsymbol{r})-1] \boldsymbol{r} \cdot \sin (\boldsymbol{Q} \cdot \boldsymbol{r}) d \boldsymbol{r}
$$

where $g(\boldsymbol{r})$ is a function proportional to the radial distribution function of the scattering bodies, $N$ is their number density, and $\xi$ is a characteristic interference distance. Consequently the structure factor depends on the degree of local order in the sample and, hence, it can be used to gain information about the relative positions of the scattering bodies by fitting the observed scattering data with one of the many approximate forms of $S(Q, \xi)$ that have been developed.

In summary, the differential scattering cross-section in a SANS experiment can be finally expressed by

$$
\frac{d \sigma}{d \Omega}(Q)=N_{p} V_{p}^{2} \cdot \Delta \eta_{n u c l}^{2} \cdot|F(Q, R)|^{2} \cdot S(Q, \xi)+B_{i n c}
$$

where $\Delta \eta_{\text {nucl }}^{2}$ is the neutron scattering nuclear contrast, $F(Q, R)$ is the form factor, $S(Q \xi)$ is the interparticle structure factor, and $B_{i n c}$ is the (isotropic and incoherent) background signal. 


\subsubsection{Magnetic Scattering}

The nuclear contrast described in the previous section is not the only contribution to the total scattering intensity in a SANS experiment since the interaction between the magnetic moment of the neutron and the magnetic field in an atom, with unpaired electronic spins or non-zero angular momentum, may give rise to magnetic scattering whose intensity is comparable to the nuclear one $20 \mid$.

The magnetic neutron scattering is a complex process that is often approximated by considering the neutron as a magnetic dipole travelling in a non-uniform magnetic field produced by a second dipole (electronic spin or angular magnetic moment). This situation

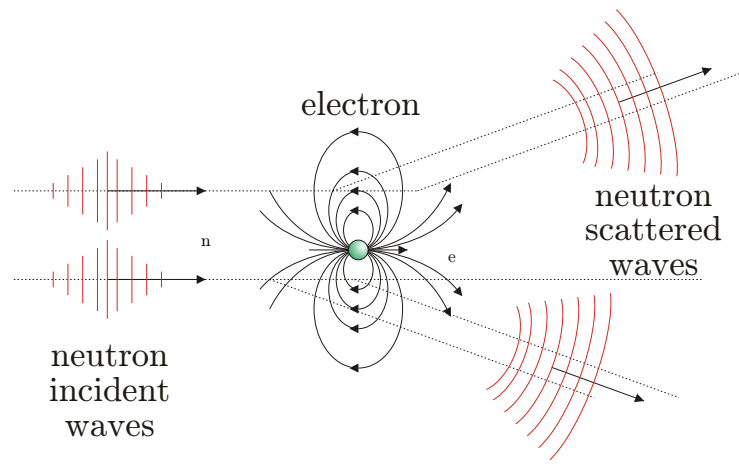

Figure 5.12. Schematic representation of the scattering process of two incident neutrons in a non-uniform magnetic field generated by a magnetic dipole.

is schematically presented in figure 5.12 .

Assuming that the magnetic neutron-electron interaction can be described through the Hamiltonian

$$
\mathscr{H}=-\mu_{n} \cdot \boldsymbol{H}_{e}
$$

where $\mu_{n}$ is the neutron magnetic moment and $\boldsymbol{H}_{e}$ is the magnetic field produced by the electron moment $\mu_{e}$, it can demonstrated that the amplitude of the magnetic scattering cross section is determined, in analogy with the nuclear scattering, by a magnetic scattering length density difference $\Delta \eta_{\text {magn }}$ (or magnetic contrast) and a magnetic form factor $F_{\text {magn }}(Q)$ 20,110

$$
\frac{d \sigma_{\text {magn }}}{d \Omega}(Q) \propto \Delta \eta_{\text {magn }}^{2} \cdot\left|F_{\text {magn }}(Q)\right|^{2}
$$

The magnetic contrast results to be proportional to 20

$$
\Delta \eta_{\text {magn }} \propto \mu_{n} \cdot\left[\hat{\mathbf{Q}} \times\left(\mu_{e} \times \hat{\mathbf{Q}}\right)\right]
$$

where $\hat{\mathbf{Q}}$ is the unitary scattering vector $(\hat{\mathbf{Q}}=\boldsymbol{Q} / Q)$. If the scattering body is not a single spin but a set of scatterers with magnetization $\boldsymbol{M}$, equation 5.44 becomes

$$
\Delta \eta_{\text {magn }} \propto \mu_{n} \cdot[\hat{\mathbf{Q}} \times(\boldsymbol{M} \times \hat{\mathbf{Q}})]
$$


The magnetic form factor $F_{\text {magn }}(Q)$ is again expressed by the relation

$$
\left|F_{\text {magn }}(Q)\right|^{2}=\frac{1}{V^{2}(\xi)}\left|\int_{V(\xi)} \exp [i f(\boldsymbol{Q} \cdot \boldsymbol{R})] d \boldsymbol{R}\right|^{2}
$$

where $\xi$ is a magnetic "shape parameter" (magnetic correlation length)

The expression of the magnetic contrast given by equation 5.45 can be significantly approximated considering the relative orientations of vectors $\boldsymbol{Q}$ and $\boldsymbol{M}$ in a real SANS experiment (see figure 5.13). Assuming that the magnetization lies along the $\mathrm{x}$-axis, it

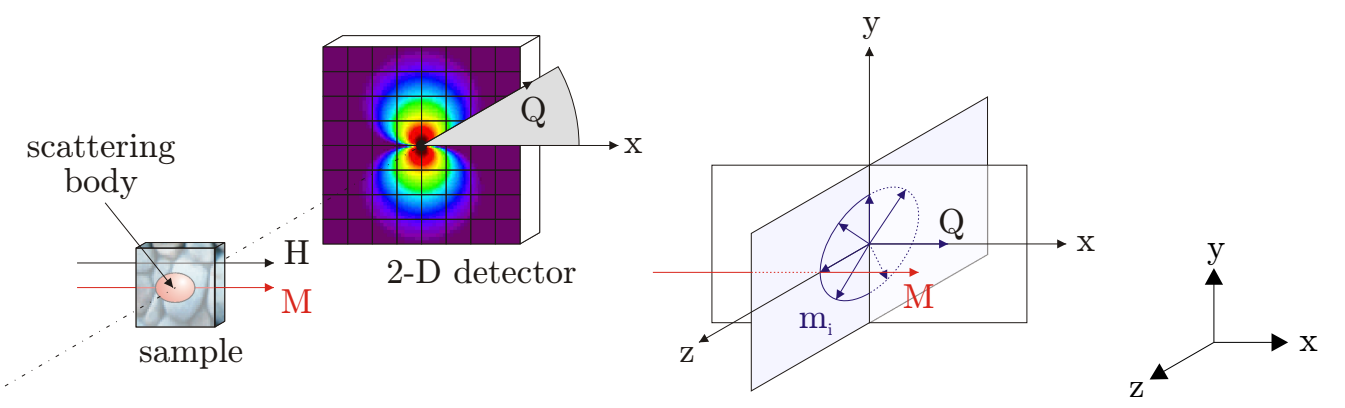

Figure 5.13. Relative orientations of the scattering vector $\boldsymbol{Q}$ and magnetization $\boldsymbol{M}$ in a SANS experiment with a magnetic target sample.

results

$$
\hat{\mathbf{Q}} \times(\boldsymbol{M} \times \hat{\mathbf{Q}})=M \cdot \sin (\alpha)
$$

therefore, the SANS intensity scattered by a magnetic body and measured in a $2-\mathrm{D}$ detector shows an angular dependence given by a factor $\sin ^{2}(\alpha)$ which is therefore zero for $\boldsymbol{Q} \| \boldsymbol{M}(\alpha=0)$. Beyond the contribution of the uniformly magnetized body, the non-uniform magnetization at its boundaries may also give rise to an additional magnetic scattering. In fact, as schematically presented in figure 5.13 (right), any magnetic moments $m_{i}$ in a plane perpendicular to the vector $Q\left(m_{i}^{\perp}\right.$ may represent, for example, domain walls in ferromagnets, grain boundaries in magnetic nanocrystalline materials, or magnetization distribution close to defects, etc.) is then responsible, according to equation 5.44, for a non-zero magnetic scattering in the directions $\boldsymbol{Q} \| \boldsymbol{M}$. The relative magnetic contrast results from the expression

$$
\Delta \eta_{\text {magn }}=\sum_{i=1}^{N} \frac{c_{i} m_{i}^{\perp}}{\Omega_{i}}
$$

where $c_{i}$ is the concentration, $\Omega_{i}$ the volume and $m_{i}^{\perp}$ the projection on the plane perpendicular to the scattering vector $\boldsymbol{Q}$ of any magnetic moment $m_{i}$.

\footnotetext{
${ }^{(8)}$ For magnetically isolated scattering bodies, $\xi$ is equal to their mean size while, for non-isolated ones, $\xi$ represents the distance below which the magnetic moments are correlated and show magnetic collective order (i.e., domain size in ferromagnetic samples).
} 


\subsubsection{Separation of nuclear and magnetic scattering}

The total contrast resulting from the interaction between neutron beam and a compositional and magnetic heterogeneous sample is in general the sum of nuclear and of magnetic contrasts, arising from the fact that the neutrons can monitor the spatial variations of both nuclear and magnetic scattering length densities [157]. Thus the differential scattering cross section for an unpolarized neutron beam ${ }^{(9)}$ can be expressed as the sum of two terms 106, 167

$$
\frac{d \sigma}{d \Omega}(Q)=\frac{d \sigma_{n u c l}}{d \Omega}(Q)+\frac{d \sigma_{m a g n}}{d \Omega}(Q)
$$

and thus

$$
\frac{d \sigma}{d \Omega}(Q)=\Delta \eta_{n u c l}^{2} \cdot P_{n u c l}^{2}(Q)+\Delta \eta_{\text {magn }}^{2} \sin ^{2}(\alpha) \cdot P_{m a g n}^{2}(Q)
$$

where $\Delta \eta_{\text {nucl }}^{2}$ and $\Delta \eta_{\text {magn }}^{2}$ are again the nuclear and magnetic contrasts terms, respectively, while $P_{\text {nucl }}(Q)$ and $P_{\text {magn }}(Q)$ are the nuclear and magnetic structure functions $(10)$, Experimentally, the two terms cannot be easily separated in a direct way. Indirectly, however, the dependence in equation 5.50 of the cross section from the term $\sin ^{2}(\alpha)$ and of the magnetic form factor $P_{\text {magn }}(Q)$ from the strength of an external magnetic field allow an angular analysis of the scattered intensity detected as two-dimensional (2-D) pattern and permit to extract information on the magnetic form factor $P_{m a g n}(Q)$ as well as on the nuclear form factor $P_{n u c l}(Q)$.

Assuming that the scattering arises from bodies of mean size $R$ which show both nuclear and magnetic contrasts, and magnetic correlation effects on a distance $\xi$, the cross section becomes 106

$$
\frac{d \sigma}{d \Omega}(Q)=\left[\Delta \eta_{\text {nucl }}^{2}+\Delta \eta_{m a g n}^{2} \sin ^{2}(\alpha)\right] \cdot P^{2}(Q, R)+\Delta \eta_{m a g n}^{2} \sin ^{2}(\alpha) \cdot P_{c o r r}^{2}(Q, \xi)
$$

Forcing the magnetization vectors to a common alignment by applying a sufficiently strong external field, the magnetic scattering is zero in the $\boldsymbol{Q}$-direction parallel to $\boldsymbol{H}(\alpha=0)$, and follows the $\sin ^{2}(\alpha)$ law for other directions as expressed by equation 5.50 . This leads to an anisotropic scattering on the $2-\mathrm{D}$ detector (see figure 5.14), where the scattering parallel to the external field is purely nuclear and the scattering perpendicular to the field is the sum of the nuclear and magnetic scattering (i.e., due to any compositional and magnetic difference between each body and its boundary). Magnetic correlations between the scattering bodies give in this case no contrast since, under a strong magnetic field, the forced common magnetic alignment extends to such large distances that it can no longer be resolved by SANS: $Q_{\max } \cdot \xi \ll 1$ and, hence, $P_{\text {corr }}(Q, \xi) \approx 0$. After performing an

\footnotetext{
${ }^{(9)}$ The spins of the neutrons in an unpolarized beam are randomly distributed in all directions.

${ }^{(10)} P^{2}(Q)$ structure function represents here the product of form and structure factors: $F^{2}(Q) \cdot S(Q)$, unless an opportune pre-factor $\left(N_{p} V_{p}^{2}\right)$ in accordance with equation 5.41 ( $B_{\text {inc }}$ is here assumed to be equal to zero).
} 


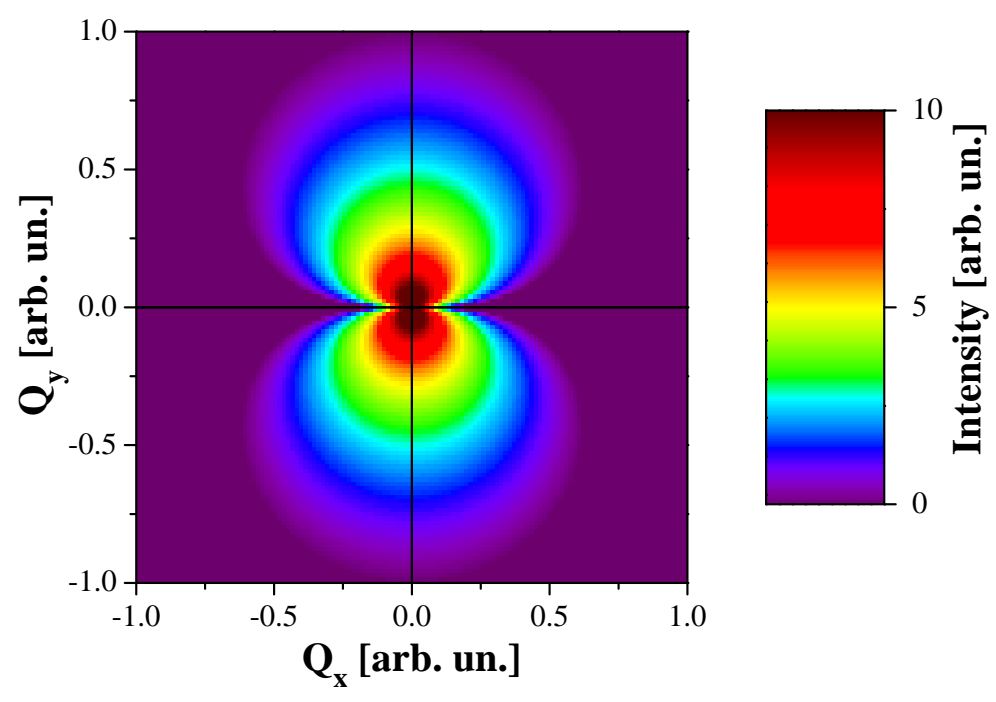

Figure 5.14. Simulated magnetic scattering intensity showing the anisotropic behavior arising from the angular dependence of the magnetic contrast: $\Delta \eta_{\text {magn }}^{2} \propto \sin ^{2}(\alpha) . \quad \boldsymbol{M}$ lies along the $\mathrm{x}$-axis. The form factor is here assumed to be $P^{2}(Q, R) \propto 1 / Q^{4}$ (spherical scatterers).

angular average in the detector plane, the radial scattering cross section in a saturating magnetic field can be rewritten as

$$
\left\langle\frac{d \sigma}{d \Omega}(Q)\right\rangle^{H=H_{\max }}=\Delta \eta_{\text {nucl }}^{2} \cdot P_{\text {nucl }}^{2}(Q)+\frac{1}{2} \Delta \eta_{\text {magn }}^{2} \cdot P_{\text {magn }}^{2}(Q)
$$

where, considering the magnetization vector of all moments fixed in the direction of the external field, the average of $\sin ^{2}(\alpha)$ yields

$$
\left\langle\sin ^{2}(\alpha)\right\rangle=\frac{1}{2}
$$

Therefore, the angular analysis of the scattered neutrons permits to separate the nuclear and the magnetic contributions considering the relations

$$
\begin{gathered}
\frac{d \sigma_{\text {nucl }}}{d \Omega}=\left\langle\frac{d \sigma}{d \Omega}\right\rangle_{\boldsymbol{Q} \| \boldsymbol{H}}^{H=H_{\max }} \\
\frac{d \sigma_{\operatorname{magn}}}{d \Omega}=2 \cdot\left(\left\langle\frac{d \sigma}{d \Omega}\right\rangle^{H=H_{\max }}-\left\langle\frac{d \sigma}{d \Omega}\right\rangle_{\boldsymbol{Q} \| \boldsymbol{H}}^{H=H_{\max }}\right)
\end{gathered}
$$

since

$$
\left\langle\sin ^{2}(\alpha)\right\rangle_{\boldsymbol{Q} \| \boldsymbol{H}}^{H=H_{\max }}=0
$$

Without external field, on the contrary, correlation effects may strongly contribute to the neutron scattering assumed that the correlation lengths lie between the resolution limits of the SANS instrument. In this case they can be successfully measured by 
SANS 104 106. If the correlated bodies have random magnetic orientations (and, hence, the $2-\mathrm{D}$ scattering pattern is isotropic), the radial scattering cross section becomes

$$
\left\langle\frac{d \sigma}{d \Omega}(Q)\right\rangle^{H=0}=\left(\Delta \eta_{\text {nucl }}^{2}+\frac{2}{3} \Delta \eta_{\text {magn }}^{2}\right) \cdot P^{2}(Q, R)+\frac{2}{3} \Delta \eta_{\text {magn }}^{2} \cdot P_{\text {corr }}^{2}(Q, \xi)
$$

where the first two terms arise again from the contrast of scattering bodies against their boundaries and the third from correlation effect:(11). Combining the previous three relations and multiplying by opportune factors, it results

$$
\frac{d \sigma_{\text {corr }}}{d \Omega}(Q)=\frac{3}{2}\left(\left\langle\frac{d \sigma}{d \Omega}\right\rangle^{H=0}-\frac{1}{3}\left\langle\frac{d \sigma}{d \Omega}\right\rangle_{\boldsymbol{Q} \perp \boldsymbol{H}}^{H=H_{\max }}+\frac{4}{3}\left\langle\frac{d \sigma}{d \Omega}\right\rangle^{H=H_{\max }}\right)
$$

from which the mean correlation length $\xi$ can be extracted by fitting the $Q$-dependence of $P_{\text {corr }}(Q, \xi)$ with one of the known expression for the form/structure factors (see appendix B).

\subsubsection{Polarized Small Angle Neutron Scattering (pSANS)}

A second possibility to resolve magnetic from nuclear scattering consists in the use of a polarized neutrons beam whose spins can have only two possible orientations ( $\operatorname{spin-up~} n^{+}$ and spin-down $n^{-}$) relative to a fixed direction 147, 171, 172.

In a pSANS experiment, the samples are maintained in a constant magnetic field $\boldsymbol{H}$ which is applied parallel to the specimen plane and normal to the neutron beam (Fig. 5.15). The vector $\boldsymbol{H}$ defines the direction of polarization for both the incident and scattered neutrons. In general, devices for reversing the polarization (the so-called flippers) are usually situated before and after the sample and are called polarizer and analyzer, respectively. A flipper is basically a crystal with zero reflectivity for one of the two neutron spin states $\left(n^{+}\right.$and $\left.n^{-}\right)$and it can be switched on/off achieving a polarization of the incoming neutrons with an efficiency $\varepsilon$ of about $90 \%$. With both flippers off, the incident beam is in the $n^{+}$state and only those neutrons which are scattered without change of spin will reach the detector. We can thus measure a cross section $[d \sigma / d \Omega]^{++}$. With the polarizer on, the incident beam is in the $n^{-}$state but the analyzer (off) will reflect only $n^{+}$neutrons, so that we measure a spin-flip $[d \sigma / d \Omega]^{-+}$cross section. Similarly, inverting the switching of the flippers, we measure a $[d \sigma / d \Omega]^{+-}$cross section, and with both polarizer and analyzer on, we measure the $[d \sigma / d \Omega]^{--}$cross section. We can therefore think in terms of four partial cross sections and of spin-flip $(S F)$ scattering $\left([d \sigma / d \Omega]^{+-}\right.$, $\left.[d \sigma / d \Omega]^{-+}\right)$and non-spin-flip $(N S F)$ scattering $\left([d \sigma / d \Omega]^{++},[d \sigma / d \Omega]^{--}\right)[71,125]$.

However, for the samples under investigation in this work, it is not of interest the analysis of the polarization state of the scattered neutrons but rather the effect of the

\footnotetext{
${ }^{(11)}$ The pre-factor $2 / 3$ of the magnetic terms results now from averaging $\sin ^{2}(\alpha)$ over arbitrary magnetization directions 144 .
} 
incoming neutron spins state on the total scattering cross section. Therefore the analyzer was not used and the intensity collected on the 2-D detector contains both SF and NSF contributions depending on the polarization state of the incident neutrons: $\left([d \sigma / d \Omega]^{++}+\right.$ $\left.[d \sigma / d \Omega]^{+-}\right)$or $\left([d \sigma / d \Omega]^{--}+[d \sigma / d \Omega]^{-+}\right)$. If the magnetic moments and the neutron polarization are directed along the external field $\boldsymbol{H}$, the total cross sections as a function of the scattering vector $Q$ are given by 172

$$
\begin{aligned}
& \frac{d \sigma}{d \Omega}^{+}(Q, \alpha)={\frac{d \sigma^{++}}{d \Omega}}^{+}+\frac{d \sigma}{d \Omega}^{+-}=\Delta \eta_{n u c l}^{2} P_{n u c l}^{2}+\left(\Delta \eta_{m a g n}^{2} P_{m a g n}^{2}+\right. \\
& \left.-2 \tau \Delta \eta_{\text {nucl }} \Delta \eta_{\text {magn }} P_{\text {nucl }} P_{\text {magn }}\right) \sin ^{2}(\alpha) \\
& \frac{d \sigma^{-}}{d \Omega}(Q, \alpha)=\frac{d \sigma^{-+}}{d \Omega}+\frac{d \sigma^{--}}{d \Omega}=\Delta \eta_{n u c l}^{2} P_{n u c l}^{2}+\left(\Delta \eta_{m a g n}^{2} P_{m a g n}^{2}+\right. \\
& \left.+2 \tau \varepsilon \Delta \eta_{\text {nucl }} \Delta \eta_{\text {magn }} P_{\text {nucl }} P_{\text {magn }}\right) \sin ^{2}(\alpha)
\end{aligned}
$$

where $\alpha$ is in this case the azimuthal angle between $\boldsymbol{H}$ and $\boldsymbol{Q}, P_{\text {nucl }}$ and $P_{\text {magn }}$ are the nuclear and the magnetic form factors, respectively, and $\tau$ is the polarization of the incoming neutron beam which is defined by

$$
\tau=\frac{\left(N^{+}-N^{-}\right)}{\left(N^{+}+N^{-}\right)}
$$

where $N^{+}$is the number of neutrons with spin antiparallel, and $N^{-}$the number of neutrons with spin parallel to $\boldsymbol{H}^{(12)}$.

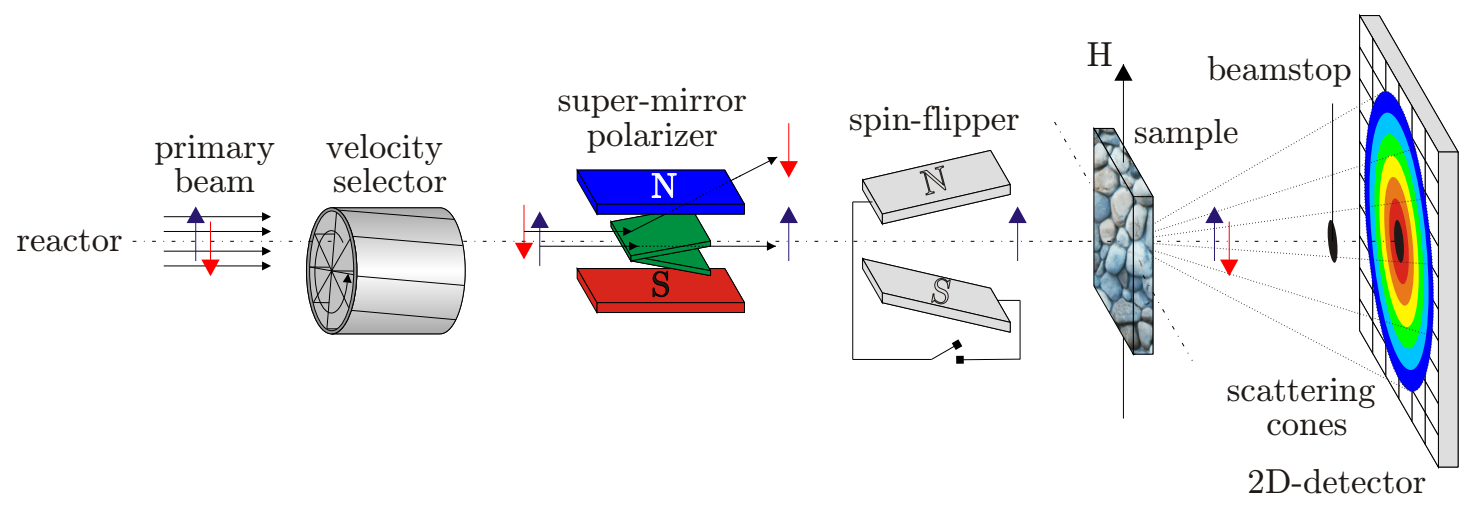

Figure 5.15. Schematic view of the experimental set-up which was used for the polarized small-angle neutron scattering measurements: the magnetic field $H$ is parallel to the sample plane and normal to the incoming neutron beam.

The arithmetic mean of the cross sections $[d \sigma / d \Omega]^{+}$and $[d \sigma / d \Omega]^{-}$corresponds to the cross section of a non-polarized beam seen in equation 5.50 because both polarization

\footnotetext{
${ }^{(12)}$ The polarization of the beamline $\mathrm{NG}-3-30 \mathrm{~m}$ at CHRNS, which we used for pSANS experiments, is approximately $95 \%$.
} 
states $n^{+}$and $n^{-}$contribute to the scattering proces: ${ }^{(13)}$

$$
\begin{gathered}
\frac{\frac{d \sigma^{+}}{d \Omega}(Q, \alpha)+\frac{d \sigma^{-}}{d \Omega}(Q, \alpha)}{2}=\frac{d \sigma}{d \Omega}(Q, \alpha)_{\text {non-polarized }}= \\
=\Delta \eta_{\text {nucl }}^{2} P_{\text {nucl }}^{2}+\Delta \eta_{\text {magn }}^{2} P_{\text {magn }}^{2} \cdot \sin ^{2}(\alpha)+ \\
-2 \tau(1-\varepsilon) \Delta \eta_{\text {nucl }} \Delta \eta_{\text {magn }} P_{\text {nucl }} P_{\text {magn }} \cdot \sin ^{2}(\alpha)= \\
\simeq \Delta \eta_{\text {nucl }}^{2} P_{\text {nucl }}^{2}+\Delta \eta_{\text {magn }}^{2} P_{\text {magn }}^{2} \cdot \sin ^{2}(\alpha)
\end{gathered}
$$

since $\varepsilon \approx 1$, while their difference represents a magnetic-nuclear cross term

$$
\begin{aligned}
& \quad \frac{d \sigma^{-}}{d \Omega}(Q, \alpha)-\frac{d \sigma^{+}}{d \Omega}(Q, \alpha)=\frac{d \sigma_{\text {cross }}}{d \Omega}(Q, \alpha)= \\
& =2 \tau(1+\varepsilon) \Delta \eta_{\text {nucl }} \Delta \eta_{\text {magn }} P_{\text {nucl }} P_{\text {magn }} \cdot \sin ^{2}(\alpha)
\end{aligned}
$$

The term $P_{\text {nucl }}$ is independent of the polarization state and, assuming a complete alignment of all magnetic moment along $\boldsymbol{H}$, entirely of nuclear origin (i.e, independent of the relative orientation of the vectors $\boldsymbol{Q}$ and $\boldsymbol{M}$ ).

The nuclear form factor $P_{\text {nucl }}^{2}$ can be determined in two independent ways from the analysis of $(d \sigma / d \Omega)^{+}$or $(d \sigma / d \Omega)^{-}$for $\boldsymbol{Q} \| \boldsymbol{H}(\alpha=0)$

$$
\eta_{n u c l}^{2} P_{n u c l}^{2}=\left\langle\frac{d \sigma^{+}}{d \Omega}\right\rangle_{\boldsymbol{Q} \| \boldsymbol{H}}=\left\langle\frac{d \sigma^{-}}{d \Omega}\right\rangle_{\boldsymbol{Q} \| \boldsymbol{H}}
$$

or from the sum signal

$$
\eta_{\text {nucl }}^{2} P_{\text {nucl }}^{2}=\frac{1}{2}\left\langle\frac{d \sigma^{+}}{d \Omega}+\frac{d \sigma^{-}}{d \Omega}\right\rangle_{\boldsymbol{Q} \| \boldsymbol{H}}
$$

The cross-term $\left(P_{\text {nucl }} \cdot P_{\text {magn }}\right)$ can be obtained by analyzing the difference signal for $\boldsymbol{Q} \perp \boldsymbol{H}$ $(\alpha=\pi / 2)$

$$
\eta_{n u c l} P_{n u c l} \cdot \eta_{m a g n} P_{m a g n}=\frac{1}{4 \tau^{2}(1+\varepsilon)^{2}} \cdot\left\langle{\frac{d \sigma^{-}}{d \Omega}}^{-}-\frac{d \sigma^{+}}{d \Omega}\right\rangle_{\boldsymbol{Q \perp \boldsymbol { H }}}
$$

and the combination of $P_{\text {nucl }}^{2}$ and $P_{n u c l} \cdot P_{\text {magn }}$ allows to reconstruct the magnetic form

\footnotetext{
${ }^{(13)}$ On the other hand, equation 5.50 can be understood in terms of equation 5.60 if we consider that an unpolarized beam has $\tau=0$.
} 
factor $P_{\text {magn }}^{2}$ according to the relation

$$
\begin{gathered}
\eta_{\text {magn }}^{2} P_{\text {magn }}^{2}=\frac{\left(\eta_{\text {nucl }} P_{\text {nucl }} \cdot \eta_{\text {magn }} P_{\text {magn }}\right)^{2}}{\eta_{\text {nucl }}^{2} P_{\text {nucl }}^{2}}=\frac{1}{4 \tau^{2}(1+\varepsilon)^{2}} \cdot \frac{\left\langle\frac{d \sigma^{-}}{d \Omega}-\frac{d \sigma^{+}}{d \Omega}\right\rangle_{\boldsymbol{Q} \perp \boldsymbol{H}}^{2}}{\frac{1}{2}\left\langle\frac{d \sigma^{+}}{d \Omega}+\frac{d \sigma^{-}}{d \Omega}\right\rangle_{\boldsymbol{Q} \| \boldsymbol{H}}} \\
=\frac{1}{2}\left\langle\frac{d \sigma^{+}}{d \Omega}+\frac{d \sigma^{-}}{d \Omega}\right\rangle_{\boldsymbol{Q \| \boldsymbol { H }}} \cdot \gamma^{2}
\end{gathered}
$$

where $\gamma$ is the ratio between the magnetic and nuclear form factors

$$
\gamma=\frac{\eta_{\text {magn }} P_{\text {magn }}}{\eta_{\text {nucl }} P_{\text {nucl }}}=\frac{1}{2 \tau(1+\varepsilon)} \cdot \frac{\left\langle{\frac{d \sigma^{-}}{d \Omega}}^{-}-\frac{d \sigma^{+}}{d \Omega}\right\rangle_{\boldsymbol{Q} \perp \boldsymbol{H}}}{\frac{1}{2}\left\langle\frac{d \sigma^{+}}{d \Omega}+\frac{d \sigma^{-}}{d \Omega}\right\rangle_{\boldsymbol{Q} \| \boldsymbol{H}}}
$$

The information obtainable by the use of polarized neutrons is basically the same that arises from unpolarized neutron scattering, namely, the magnitude of $\Delta \eta_{\text {nucl }}$ and $\Delta \eta_{\text {magn }}$, and the $Q$-dependence of $P_{\text {nucl }}(Q, R)$ and $P_{\text {magn }}(Q, \xi)$. However, pSANS shows with respect to SANS a main advantage which emerges clearly by considering the definition of the ratio $\gamma$ in equation 5.66 ; in fact, $\gamma$ is here linear in the magnetic contrast $\Delta \eta_{\text {magn }}$ and amplitude $P_{\text {magn }}(Q, \xi)$ while, in an unpolarized beam, only $\gamma^{2}$ can be directly calculated (see equation 5.54 172

$$
\gamma^{2}=2 \frac{\left\langle\frac{d \sigma}{d \Omega}\right\rangle^{H=H_{\max }}-\left\langle\frac{d \sigma}{d \Omega}\right\rangle_{\boldsymbol{Q} \| \boldsymbol{H}}^{H=H_{\max }}}{\left\langle\frac{d \sigma}{d \Omega}\right\rangle_{\boldsymbol{Q} \| \boldsymbol{H}}^{H=H_{\max }}}
$$

and consequently, $\gamma$ can be determined much more accurately by pSANS than by nonpolarized neutrons. Thus, polarized neutrons permit through $\gamma$ a more reliable evaluation of the ratio between magnetic and nuclear contrast, especially in the case when the first is much smaller than the second [173, 174].

\subsubsection{Neutron Sources}

Neutrons for small-angle (or wide-angle) scattering experiments are usually produced in two ways: by nuclear fission or by "spallation". The first method makes use of the fission reaction of ${ }^{235} \mathrm{U}$ which releases $2-3$ neutrons each fission event. The produced neutrons, except one which is needed to sustain the reaction chain, are then extracted from the reactor and sent to the beamline through special neutrons guides.

The second approach is that used in spallation neutron sources in which accelerators and synchrotrons generate intense, high-energy, proton beams that are directed in pulses 
to a target composed of heavy nuclei (for example Ta, depleted U, or liquid Hg). Provided that the protons have sufficient kinetic energy the incoming beam is able to overcome the intrinsic long-range electrostatic and short-range nuclear forces and effectively blast the target nuclei apart (spallation). 20 to 30 neutrons per pulse are released during the collision and then sent to the beamline through low-absorption and high-reflectivity guides.

Three different beamlines were used to measure small angle neutron scattering data presented in this work:

- SAND of the Intense Pulsed Neutron Source (IPNS) at the Argonne National Laboratory, Argonne (USA) 156

- SANS-I of the Swiss Spallation Neutron Source (SINQ) at the Paul Scherrer Institute (PSI), Villingen (Switzerland)

- NG-3-30 of the Center for High Resolution Neutron Scattering (CHRNS) at the National Institute of Standards and Technology (NIST), Gaithersburg (USA)

The first two were employed for unpolarized SANS, while the last was utilized for pSANS experiments.

IPNS is a pulsed spallation source that uses a narrow spectrum of polychromatic neutrons $(\Delta \lambda)$ and a time-of-flight detection technique $[77]$ to analyze neutrons scattered at vectors $Q$ between $\sim 0.004$ and $\sim 0.6 \AA^{-1}$ in just one experiment. On the contrary, CHRNS is a reactor source and SINQ a spallation source that utilize a selectable monochromatic neutron beam and a detection system based on a moveable $2-\mathrm{D}$ scintillation detector which can be positioned at different distances from the sample to cover the same range of scattering vectors as that measurable at IPNS.

The fixed instrument geometry at IPNS and the moveable configuration at CHRNS or at SINQ represent two different approaches to scan a range of the scattering vectors and, hence, to probe a certain range of length scales. Those two approaches become clearer considering the definition of the scattering vector $Q$ introduced in equation 5.22

$$
Q=\frac{4 \pi}{\lambda} \sin \left(\frac{\phi}{2}\right)
$$

At CHRNS and SINQ, $Q$ is varied by effectively scanning $\phi$ at a pre-selected value of $\lambda$ while at IPNS, $\phi$ is fixed and a range of $Q$-values is then obtained by time-sorting different $\lambda$ 's from a pulsed polychromatic incident beam as it arrives at the detector. 


\subsection{High Resolution Transmission Electron Microscopy (HRTEM)}

Transmission electron microscopy (TEM) is the most preferable technique for the direct "imaging" of a sample. Although the specimens preparation usually requires great effort, transmission electron microscopy is widely used in many different branches of science due to its capability of generating several types of image: high-resolution (HR-) bright and dark fields, electron diffraction, electron energy-loss spectroscopy, $\mathrm{x}$-ray microanalysis, and many others.

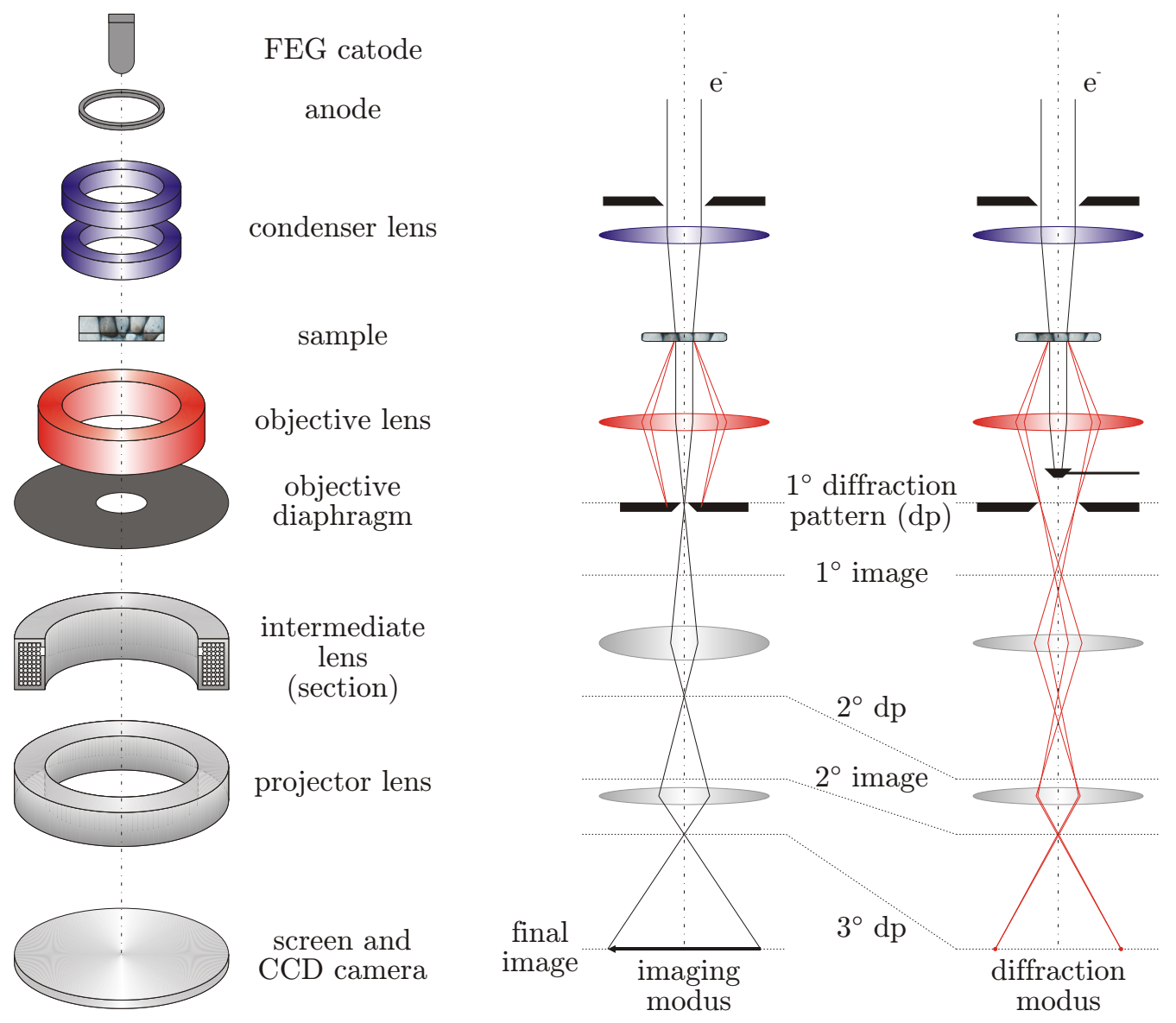

Figure 5.16. Schematic drawing of a high resolution electron microscope indicating the set of magnetic lenses (left) and the different illuminating geometries for direct imaging and diffraction modus (right).

In a conventional transmission electron microscope (see figure 5.16) a thin specimen $(5-500 \mathrm{~nm})$ is irradiated with an electron beam of uniform current density and of energy in the range $60-200 \mathrm{keV}$ (in the case of intermediate-voltage electron microscopes). A two stage condenser-lens system allows variation of the illumination and of the investigated area of the specimen, while a three- or four-stage lens system behind the specimen images and magnifies the electron-intensity distribution arising from the sample-electron 
interaction. The image is then recorded by direct exposure of a CCD camera controlled by a computer. The HRTEM investigation carried out during this work employed a Philips CM200 microscope at $200 \mathrm{kV}$.

Several types of interactions are responsible for a TEM "image" (contrast) of a specimen:

- elastic scattering

- inelastic scattering

- absorption

- inner-shell ionization

- multiple-scattering effects

- $\mathrm{x}$-ray and Auger-electron emission

In general a contrast is produced either by absorption of the electrons scattered through angles larger than the objective aperture (scattering contrast), by interference between the scattered wave and the incident wave at the image point (phase contrast), by diffraction in accordance with Bragg's law, or simply by the virtue of the inhomogeneity of the specimen (density contrast, $Z$ contrast). The interpretation of these contrasts in terms of the microstructure from which they arise is a complex task and "a science in its own right" (P. Haasen) 68.

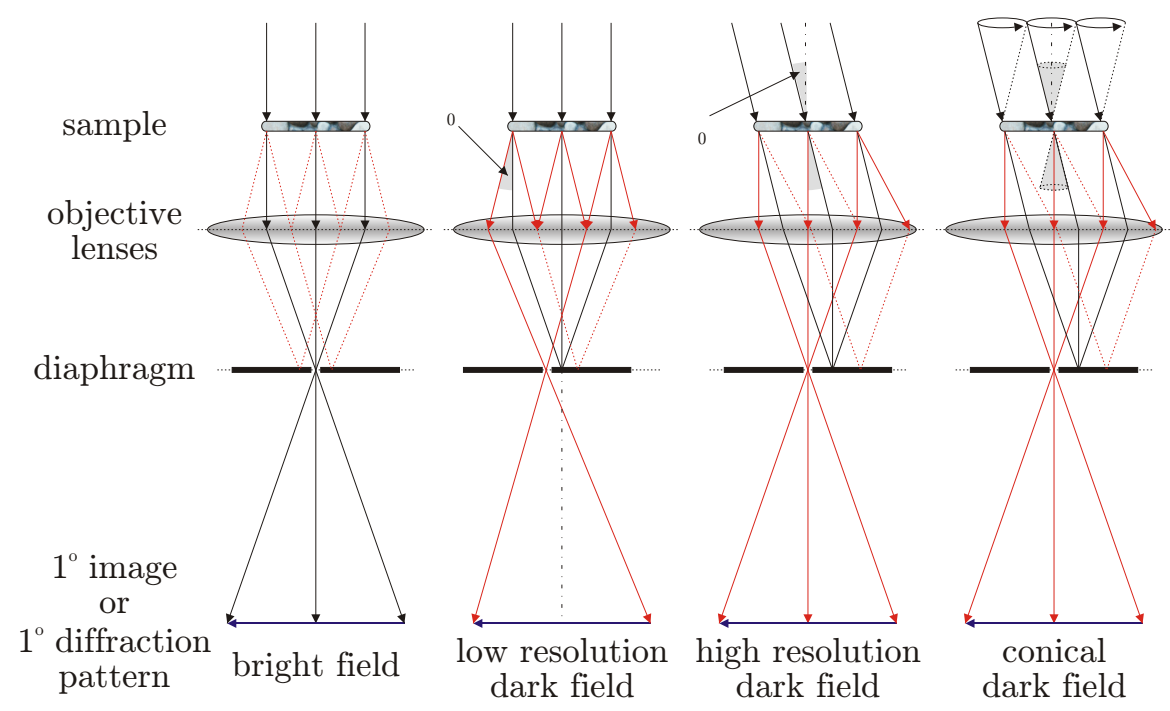

Figure 5.17. Schematic representations of the different illuminating modi in bright field, low-resolution dark field, high-resolution dark field, and conical dark field electron microscopy, respectively.

The bright field mode (BF) with a centered objective diaphragm is the typical TEM mode with which scattering contrast and diffraction contrast can be produced. A second 
mode which is widely used in the case of composites or polycrystalline materials is the dark field mode (DF). In DF mode the primary beam is intercepted in the focal plane of the objective lens. Different ways of producing dark field conditions are in use and they are schematically presented in figure 5.17. The easiest method is the so-called shifted diaphragm, in which the objective diaphragm intercepts the primary beam and the scattered electrons pass through the objective lens on off-axis trajectories. In this case the off-axis conditions are responsible for the resultant low resolution dark field image mainly due to chromatic aberration of the lenses. An alternative to this method consists in the use of a central beam stop which intercepts the primary beam but not the diffracted beams which pass through the diaphragm in a less off-axis way. The most common way to produce dark-field images is however to tilt the primary electron beam before the specimen by an angle $\alpha_{0}$ such that only the scattered beam strikes the centered diaphragm. In a similar way, this method can also be employed to image crystalline specimens with selected Bragg diffraction spots by tilting the primary beam of an angle equal to $2 \theta_{B}$, where $\theta_{B}$ is the Bragg angle of the selected diffraction beam. Asymmetries in the image which can be due also to different grain orientations in polycrystalline specimens can be avoided by changing the direction of tilt around a cone. The image produced by this method is therefore called conical dark-field.

\subsubsection{Sample Preparation}

Due to the strong electron-specimen interaction and to absorption effects, a sample suitable for TEM investigation must be very thin. Special preparation techniques are therefore needed. In the case of the $\mathrm{Nd}_{60} \mathrm{Fe}_{30} \mathrm{Al}_{10}$ samples, the specimens were first thinned by mechanical polishing and then by Ar-ion beam $(4.5 \mathrm{kV})$ in a GATAN DuoMill 500 set up with a sample holder cooled by liquid nitrogen.

\subsection{Differential Scanning Calorimetry (DSC)}

Differential scanning calorimetry (DSC) is a technique used to thermally characterize a wide class of materials: metals, alloys, ceramics, polymers. Phase transformation temperatures, enthalpies of transformation and thermal hysteresis data of a sample can be determined by measuring the heat flow of the sample against that of a reference material, such as aluminum, over a range of temperatures or time.

The DSC device used to characterize the $\mathrm{Nd}_{60} \mathrm{Fe}_{30} \mathrm{Al}_{10}$ samples is a Perkin-Elmer DSC7 with power compensation. Compared to a traditional DSC, the DSC7 is a more sensible device thanks to the possibility to measure the heat released (or absorbed) by a sample by almost totally compensating it with a decrease (or an increase) of electric energy produced from an adjustable Joule's heater. 
A schematic drawing of the measuring system of a DSC7 is presented in figure 5.18 . The calorimeter consists of two identical microfurnaces made of platinum-iridium alloy, each of which contains a temperature sensor (platinum resistance thermometer plate) and a heating resistor (platinum wire). Both microfurnaces are thermally decoupled and positioned in an aluminum block of constant temperature. During heating-up, the same

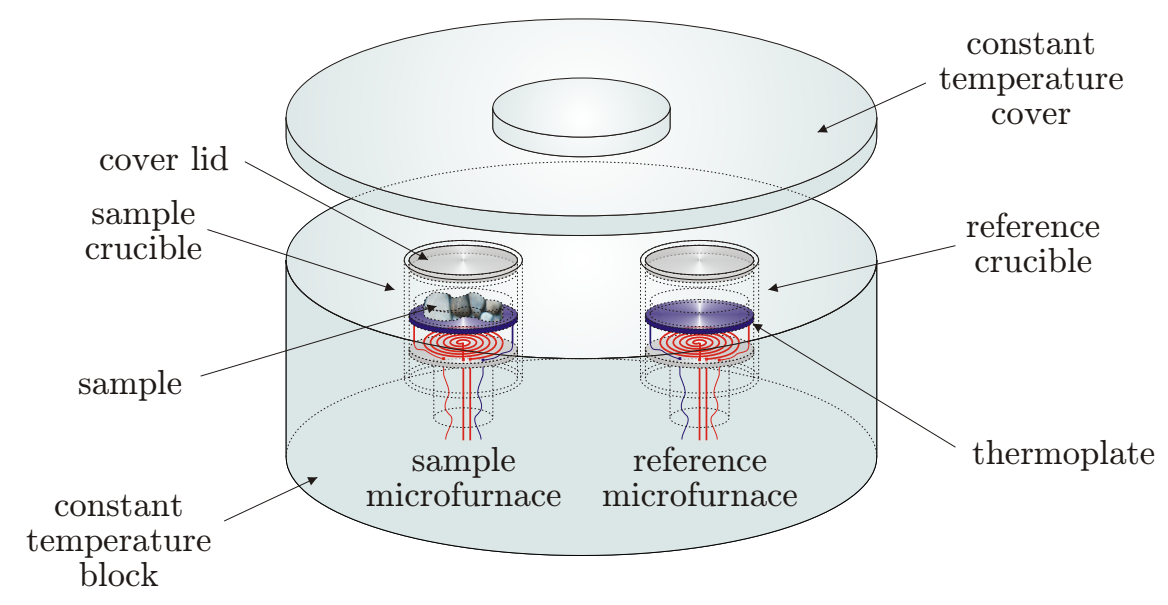

Figure 5.18. Power compensation DSC7 set-up. The device consists of two microfurnaces each of which is about $9 \mathrm{~mm}$ in diameter and can contain a sample/reference crucible. The temperatures of the microfurnaces are controlled independently by two heating wire and two resistance thermometer-plates.

heating power is supplied to both microfurnaces via a control circuit (control unit) in order to change their mean temperature in accordance with a heating rate set by software (computer unit). If there is ideal thermal symmetry, the temperature of both microfurnaces is always the same and $d(\Delta T) / d t=0$. When an asymmetry occurs, for example as a result of a sample reaction, a temperature difference $\Delta T^{\prime}$ results between sample and reference microfurnace. This difference is compensated by the DSC7 by increasing or decreasing proportionally the heating power of a quantity $\Delta P$. But $\Delta T^{\prime}$ cannot be completely compensated because of the proportional controller ${ }^{(14)}$ and a remaining temperature difference $\Delta T$ is still present. The relation which links $\Delta P$ to $\Delta T$ can be expressed by:

$$
\Delta P=-k_{1} \cdot \Delta T
$$

where $k_{1}$ is a factory-set fixed constant which depends only on the characteristic of the proportional controller. In the same way a heat flow rate $\dot{Q}_{\text {measured }}$ is assigned to the real

\footnotetext{
${ }^{(14)}$ The proportional controller cannot react instantaneously to a thermal change and a remnant heat release arises.
} 
measurement signal $\Delta T$ taking into account the relation $(15)$,

$$
\dot{Q}_{\text {measured }}=\left(\frac{d Q}{d t}\right)_{\text {measured }}=-k_{2} \cdot \Delta T
$$

where $k_{2}$ is a constant which can be determined by one calibration measurement. The true heat flow rate signal exchanged with the sample must also be determined by a calorimetric measurement ${ }^{(16)}$ and is normally expressed by the equation

$$
\dot{Q}_{\text {true }}=K_{Q} \cdot \dot{Q}_{\text {measured }}
$$

Finally, the time integral over the compensating power gives the heat $Q$ which was consumed or released in the sample

$$
Q=\int \dot{Q}_{\text {measured }} d t=-\int k_{2} \cdot \Delta T d t=-\int K_{Q} \cdot k_{2} \cdot \Delta T d t
$$

and, taking into account the heating rate relation,

$$
\frac{d T}{d t}=\beta
$$

the heat capacity of the sample and the enthalpy release of the reaction can also be calculated as

$$
\begin{gathered}
\frac{\dot{Q}}{\beta}=\frac{\frac{d Q}{d t}}{\frac{d T}{d t}}=\frac{d Q}{d T}=C_{p} \\
\Delta H_{\text {reaction }}=\int_{\Delta t_{\text {reaction }}} \dot{Q} d t=\int_{\Delta T_{\text {reaction }}} \frac{\dot{Q}}{\beta} d T
\end{gathered}
$$

\subsection{High Energy Wide Angle X-Ray Scattering (HE- WAXS)}

Among the existing methods (XRD, HRTEM, FIM, Atom probe, etc.) which permit to investigate the structure of a specimen and its evolution during in situ thermal treatments, the high-energy $\mathrm{x}$-ray diffraction $(\mathrm{HE}-\mathrm{XRD})$ is a powerful tool due to its capability to access a wide range of length scales $\left(Q \in[1,10] \AA^{-1}\right)$ in few seconds and nondestructively $3,127,134$. With the recent advent of high-brilliance third generation $\mathrm{x}$-rays

\footnotetext{
${ }^{(15)}$ The negative sign is introduced because a temperature increase $(\Delta T>0)$ appears as a consequence of an exothermic process which has conventionally $\dot{Q}<0$.

${ }^{(16)}$ For this scope the heat flow released during the melting of a pure zinc or indium sample is generally used.
} 
synchrotrons, a transmission geometry has been developed which allows simultaneous collection of small-angle and wide-angle $\mathrm{x}$-ray scattering (SAXS and WAXS, respectively) in thin films and bulk materials, significantly extending traditional XRD methods based on reflection geometries ${ }^{(17)}$.

HE-WAXS on $\mathrm{Nd}_{60} \mathrm{Fe}_{30} \mathrm{Al}_{10}$ slow-cooled, bulk, splat-cooled samples, and thin films was performed at the Advanced Photon Source (APS) at Argonne National Laboratory on the 1-ID beamline which is schematically reported in figure 5.19 .

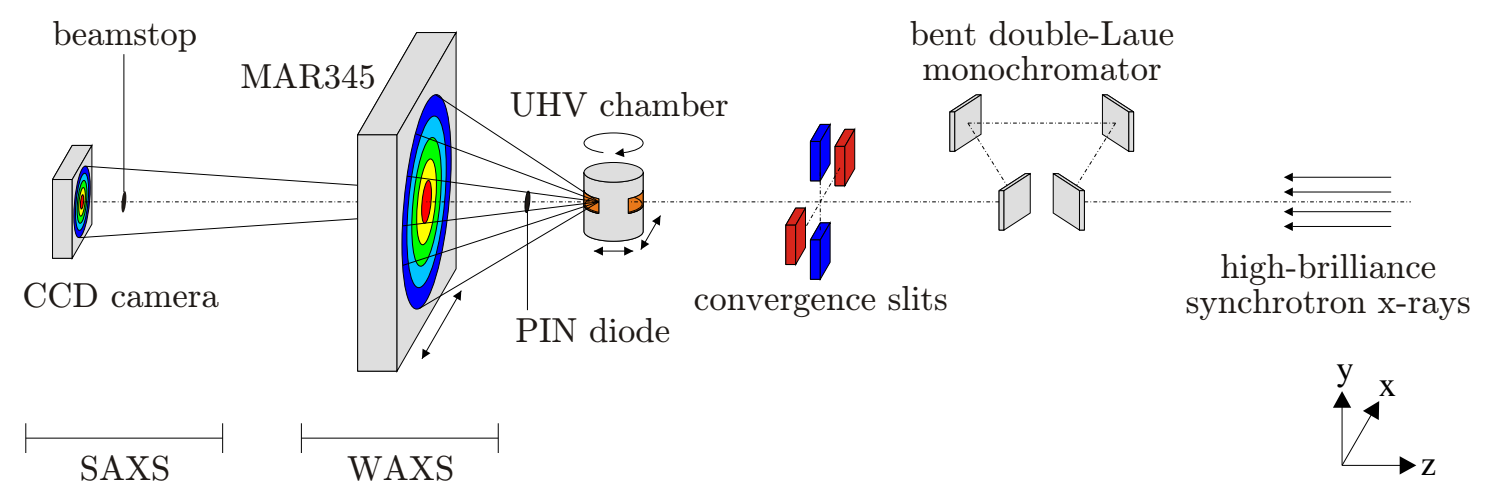

Figure 5.19. Schematic drawing of the 1-ID beamline at the Advanced Photon Source (APS) at Argonne National Laboratory. A bent double-Laue monochromator, convergent double slits and a UHV chamber have been employed for the in situ characterization of the specimens. A MAR345 and an x-ray CCD camera has been used for the acquisition of the diffuse scattered beam.

A bent double-Laue monochromator has been used to provide a high-energy beam $(E=80.715 \mathrm{keV}, \lambda=0.1535 \AA)$ with a flux density of $I \simeq 10^{12}$ photons $\cdot \mathrm{s}^{-1} \cdot \mathrm{mm}^{-2}$ while a double set of slits has been used to obtain a transverse beam size of about $50 \times 50 \mu \mathrm{m}^{2}$. The transmission coefficients of the specimens have been measured with a PIN diode which permitted to re-scale the measured pattern relatively to the transmitted intensity. Several complete Debye-Scherrer diffraction rings (see for example figure 6.2 have been recorded with a MAR345 on-line imaging plate (345 mm diameter, $150 \mu \mathrm{m}$ pixel size) characterized by a fluorescent $\mathrm{x}$-rays sensible film which can be read in scanning mode after each exposure by an optical diode. The fluorescence intensity (proportional to the intensity of the scattered X-ray beam) of each pixel can be hence converted to a current and its value is recorded in a matrix of $2300 \times 2300$ points $(\sim 5 \mathrm{M}$ pixels $)$ which can be read by computer for the necessary data reduction. The time-out of MAR345 allows to take a complete scan every $30 / 60 \mathrm{~s}$.

The fast acquisition method at the 1-ID makes possible the investigation of structural

\footnotetext{
${ }^{(17)}$ The main disadvantages connected with the use of traditional diffractometers in the structural investigation of amorphous or composite specimens regard the reduced $\mathrm{x}$-ray penetration depth (order of $\mu \mathrm{m}$ ) and the generally low intensity of the diffracted beam which limit the reliability of the XRD method when applied to this class of materials.
} 
evolution during in situ thermal treatments of metastable materials as intrinsic composites. For this scope several specimens have been heated from room temperature up to $814 \mathrm{~K}$ with a constant rate and a complete diffraction pattern was taken every $4 / 5 \mathrm{~min}$. The maximal sample thickness has been limited to about $0.5 \mathrm{~mm}$ by the absorbtion of $\mathrm{Nd}_{60} \mathrm{Fe}_{30} \mathrm{Al}_{10}$ and, hence, by the statistical necessity to have a transmission factor greater than $30 \%$. The specimens have been mounted in a copper sample holder whose temperature was set by two boron-nitride heaters and controlled by a thermocouple element. The experimental setup has been maintained at a pressure of about $10^{-5}$ mbar in an UHV chamber ${ }^{(18)}$ to prevent oxidation (see figure 5.20 .
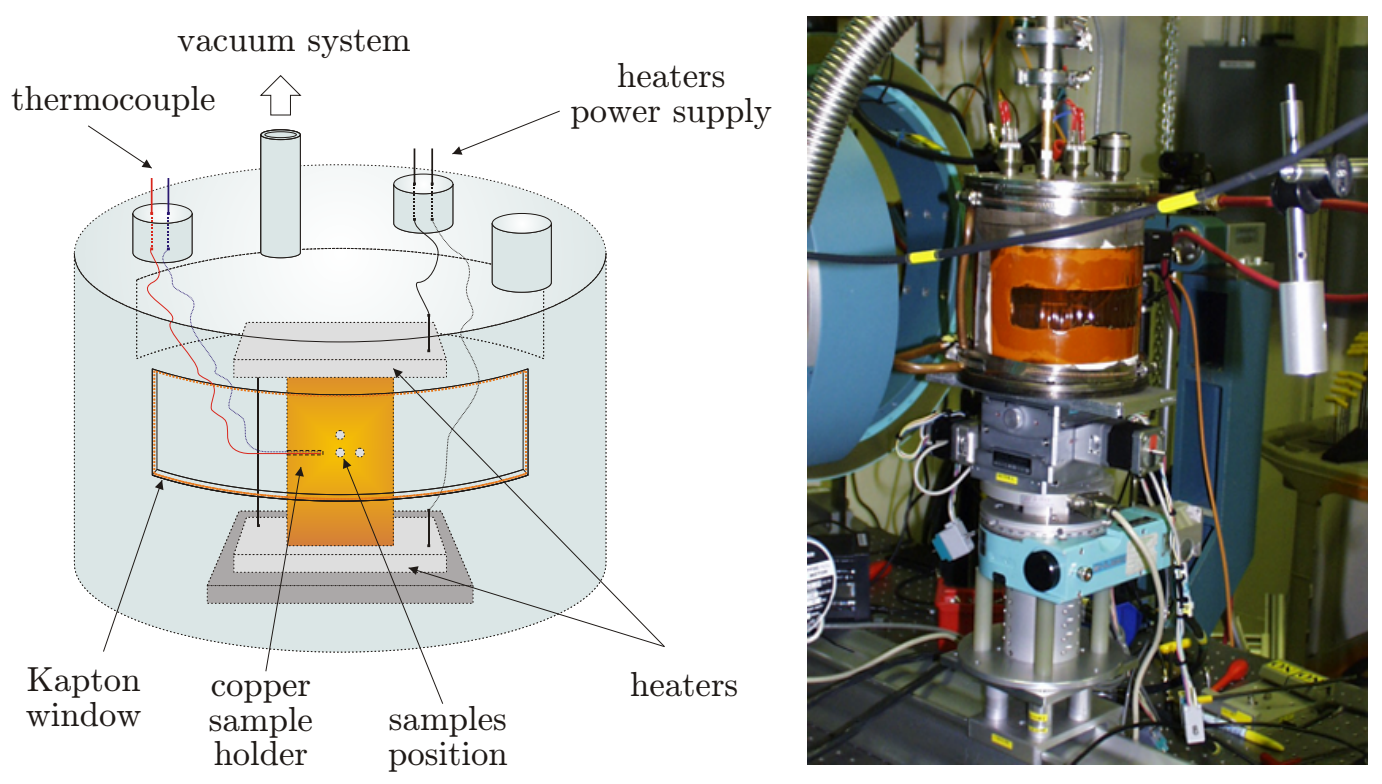

Figure 5.20. (Left) Schematic drawing of the copper sample holder used during in situ thermal treatments at 1-ID WAXS beamline. (Right) Picture of the UHV chamber in which the holder has been maintained to prevent the sample from oxidation. A XYZ translator and a rotator situated under the chamber allow to adjust the position of the sample relative to the $\mathrm{x}$-ray beam.

Before sample exposure, a calibration sample $\left(\mathrm{CeO}_{2}\right.$ powder, also called ceria) has been measured to determine the beam's center, sample-detector distance and to correct for tilting of the detector referencing to the Debye rings of the ceria (fcc, $a=5.41 \AA$ ). The 2-D WAXS pattern obtained from $\mathrm{CeO}_{2}$ is shown as example in figure 5.21.

Two-dimensional patterns have been transformed from polar to Cartesian coordinates using bins of $1^{\circ}$ and $\delta r=150 \mu \mathrm{m}$, providing 360 one-dimensional radial patterns. The

\footnotetext{
${ }^{(18)}$ The diffuse scattering arising from the thin kapton foil windows, through which the $\mathrm{x}$-ray beam entered the UHV chamber, is very small and did not play an important role within the scope of our experiment.
} 


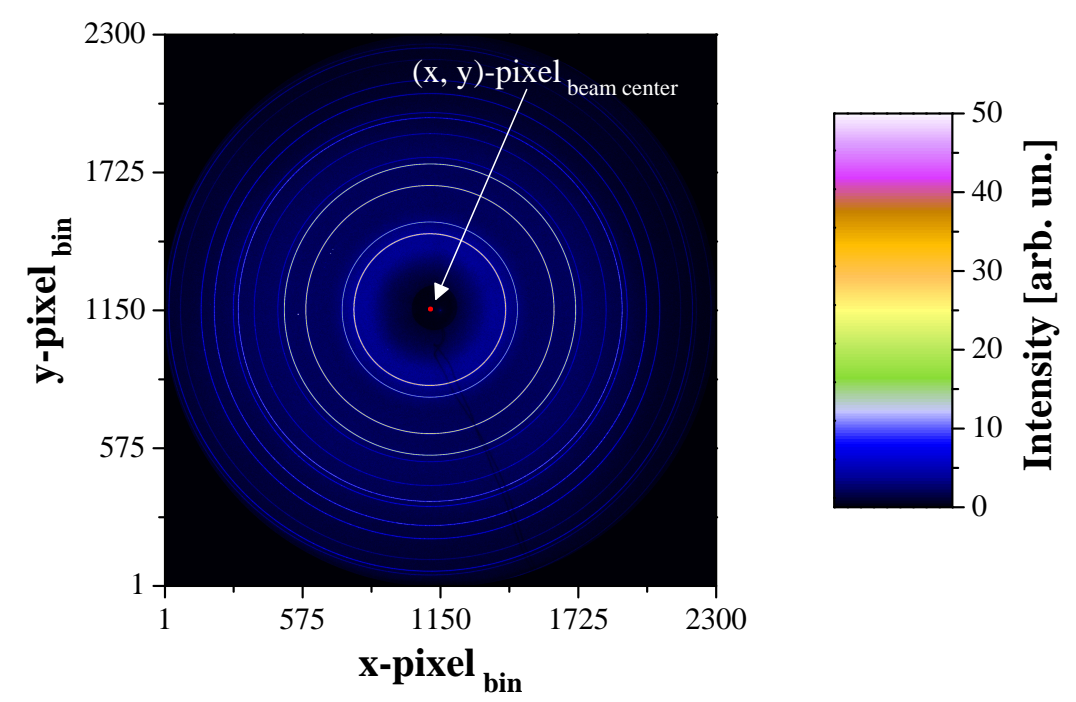

Figure 5.21. Diffuse WAXS pattern of $\mathrm{CeO}_{2}$ powder which is commonly used as WAXS calibration sample to determine the beam's center, sample-detector distance and to correct for tilting of the detector.

set of relations

$$
\begin{aligned}
x_{i} & =\left(x-\text { pixel }_{\text {bin }}-x-\text { pixel }_{\text {beam center }}\right) \cdot \delta r \\
y_{i} & =\left(x-\text { pixel }_{\text {bin }}-x-\text { pixel }_{\text {beam center }}\right) \cdot \delta r \\
Q_{i} & =\frac{4 \pi}{\lambda} \sin \left[\frac{1}{2} \arctan \left(\frac{\sqrt{x_{i}^{2}+y_{i}^{2}}}{d_{\text {sample-detector }}}\right)\right]
\end{aligned}
$$

permits to calculate the correct value of the absolute scattering vector $Q_{i}$ for each bin $i$.

In the case of intrinsic composite materials or polycrystalline samples, not showing texturing, the statistics of the WAXS measurement can be further improved by averaging the 360 curves to obtain a single $I$ vs. $Q$ diffraction pattern. Such averaging procedure was used for each sample studied in this work, as shown for example in figure 6.3 for a bulk $\mathrm{Nd}_{60} \mathrm{Fe}_{30} \mathrm{Al}_{10}$ specimen.

\subsubsection{Directions and Intensities of diffracted X-Ray Beam}

$\mathrm{X}$-ray diffraction is traditionally the most used technique for structural investigation of ordered (crystalline) and disordered (amorphous and vitreous) specimens in condensed matter physics thanks to the different information which can be easily and quite inexpensively extracted from the $\mathrm{x}$-rays diffracted from the sample.

As already well-known, a beam interacting with a crystalline sample is diffracted anisotropically producing constructive interference in directions which are given by the Bragg's angle $\theta_{B}$ (see figure 5.22

$$
n \cdot \lambda=2 d \cdot \sin \left(\theta_{B}\right)
$$




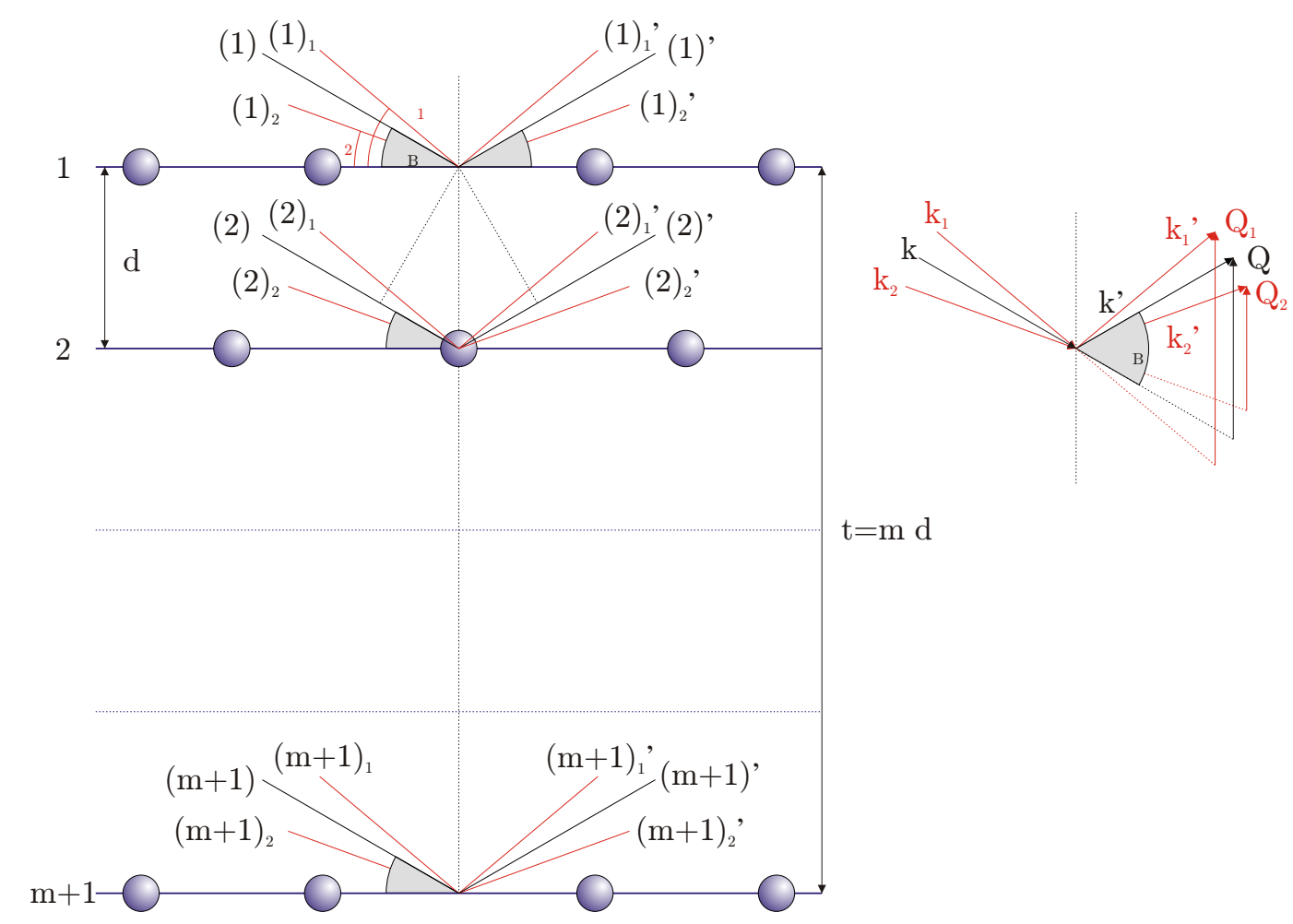

Figure 5.22. $x$-ray diffraction in non ideal conditions (beam not perfectly parallel and strictly monochromatic) on a crystal of finite size $t$.

where $d$ is the crystalline planes distance, $n$ is the order of reflection and $\lambda$ is the $\mathrm{x}$-ray wavelength. Introducing the concept of wave vector 7

$$
\begin{aligned}
\boldsymbol{k} & =\frac{2 \pi}{\lambda} \cdot \hat{i} \\
\boldsymbol{k}^{\prime} & =\frac{2 \pi}{\lambda} \cdot \hat{i}^{\prime}
\end{aligned}
$$

where $\hat{i}$ and $\hat{i}^{\prime}$ are unitary vectors expressing the direction of the rays before and after the scattering process respectively, equation 5.76 can be analogously expressed by the expression (von Laue formalism):

$$
\boldsymbol{k}-\boldsymbol{k}^{\prime}=\boldsymbol{Q}=c \cdot \frac{2 \pi}{d} \cdot \hat{n}
$$

where $c$ is an integer and $\hat{n}$ is a unitary vector of the reciprocal space $(Q=|\boldsymbol{Q}|$ is the scattering vector). The von Laue condition states that constructive interference occurs in the direction $\hat{i}^{\prime}$ provided that the change in wave vector $\boldsymbol{Q}=\boldsymbol{k}-\boldsymbol{k}^{\prime}$ is a vector of the reciprocal lattice.

If the sample is composed of crystals with a finite number $m$ of crystalline planes (crystal thickness $t=m \cdot d$ ) and the X-ray beam is not perfectly parallel and strictly monochromatic as supposed in the Bragg's and von Laue's formalisms, diffraction occurs under non ideal conditions: the incident rays do not all have the same wave vector $\boldsymbol{k}$ but 
a distribution of wave vectors which goes, for example, from $\boldsymbol{k}_{1}$ to $\boldsymbol{k}_{2}$ and it can happen that the ray $(m+1)_{1}^{\prime}$, scattered from the plane $(m+1)$, is $(m+1)$ wavelengths out of phase with $(1)_{1}^{\prime}$, scattered from the first plane, instead of the $m$ wavelengths under ideal Bragg conditions. This means that midway in the crystal there is a plane $l$ scattering a ray $l_{1}^{\prime}$ which is $(l+1 / 2)$ wavelengths out of phase with $(1)_{1}^{\prime}$ and, therefore, giving rise to destructive interference for all $(1)_{1}^{\prime},(2)_{1}^{\prime}, \ldots(m+1)_{1}^{\prime}$ rays. Hence, the intensity of the beam diffracted at

$$
Q_{1}=k_{1}^{\prime}-k_{1}
$$

for which:

$$
\left|\boldsymbol{Q}_{1}\right|=Q_{1}=\frac{2 \pi}{t}(m+1)
$$

is therefore zero. The diffracted intensity is also zero for a scattering vector $\boldsymbol{Q}_{2}$ such that the ray $(m+1)_{2}^{\prime}$ is $(m-1)$ wavelengths out of phase with $(1)_{2}^{\prime}$. The curve of the resulting diffracted intensity has the form shown in figure 5.23 (left) in contrast to figure 5.23 (right) for the hypothetical case of ideal diffraction conditions.
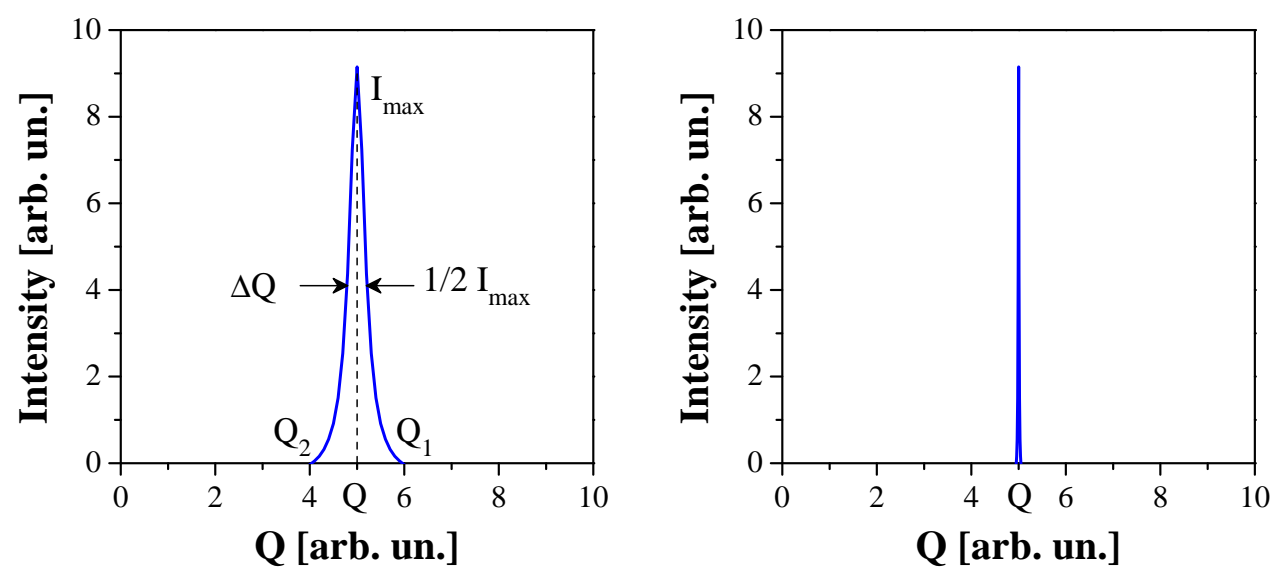

Figure 5.23. Schematic $x$-ray diffraction intensity profiles of diffracted beams ideal (right) and non ideal (left) conditions. The FWHM $\Delta Q$ permits to estimate through the Scherrer formula the mean crystalline size.

Destructive interference (zero intensity) happens therefore at wave vectors $Q_{1}$ and $Q_{2}$ which satisfy the conditions

$$
\begin{aligned}
& Q_{1}=\frac{2 \pi}{t}(m+1) \\
& Q_{2}=\frac{2 \pi}{t}(m-1)
\end{aligned}
$$

By subtraction

$$
t=\frac{4 \pi}{Q_{1}-Q_{2}}
$$

and, considering that $Q_{1}-Q_{2} \simeq 2 \Delta Q$ where $\Delta Q$ is the full width at half maximum (FWHM) of the diffracted intensity curve (see figure 5.23), the crystal size $t$ can be 
rewritten as

$$
t=\frac{2 \pi}{\Delta Q}
$$

or, assuming that the crystal has a spherical shape, the averaged crystal radius $r$ can be expressed as

$$
r=\frac{0.9 \cdot \pi}{\Delta Q}
$$

Equation 5.86 represents the analogous of the Scherrer formula expressed in terms of diffraction angle [44, p. 102] and permits to calculate the mean crystal radius $r$ from the broadening of the diffraction peaks.

Beyond the information arising from the analysis of the scattering directions, several others can be obtained from the study of the relative intensities of the diffraction peaks 44 , ch. 14]. In most of the cases such analysis has only a qualitative character which may however be useful under precise assumptions in many practical situations. According to Warren [169] and Cullity [44, the exact expression for the intensity diffracted by a single-phase powder specimen (in a diffractometer) can be schematically expressed as:

$$
I=I_{\text {incident }} \cdot f\left(F^{2}, \theta_{B}, \mu\right)
$$

where $I_{\text {incident }}$ is the intensity of the incident beam, $F$ the structure factor, $\theta_{B}$ the Bragg's angle, and $\mu$ the linear absorption coefficient. In the case of intrinsic composite materials, not a single-phase but a mixture of at least two phases gives rise to diffraction: the amorphous matrix and the crystalline precipitates. Equation 5.87 can then be simplified taking into account only a particular line of the crystalline phase and comparing it with the diffuse scattering intensity of the matrix.

Qualitatively, the area under a diffraction peak, as contributed by each phase, determines the intensity of the peak which can be written as:

$$
\begin{aligned}
I_{\text {crys }} & =\frac{c}{\mu_{m}} x \cdot Z_{\text {crys }}^{2} \\
I_{\mathrm{am}} & =\frac{c}{\mu_{m}}(1-x) \cdot Z_{\text {am }}^{2}
\end{aligned}
$$

where $c$ is a constant, $x$ is the volume fraction of the crystalline phase, $\mu_{m}$ is the averaged linear absorption coefficient of the mixture, and $Z_{i}$ is the average atomic number of the phase $i$. Measuring directly in an $\mathrm{x}$-ray pattern the percentage of crystallinity $\alpha$ expressed as:

$$
\alpha=\frac{I_{\text {crys }}}{I_{\text {crys }}+I_{\mathrm{am}}}
$$

the volume fraction $x$ in equation 5.88 results to be:

$$
x=\frac{\alpha Z_{\mathrm{am}}^{2}}{Z_{\mathrm{crys}}^{2}+(1-\alpha) Z_{\mathrm{am}}^{2}}
$$




\subsection{Annealing Methods}

In chapter 3 three different processes, which can contribute to microstructure transformations and, hence, to possible improvement or tailoring of the physical properties of a material, have been presented: nucleation, growth, and coarsening. In the solid state such processes are transformations activated by high temperature annealing and occur slowly due to relatively low solid state diffusion (compared to liquid diffusion). In this way it is possible for metastable phases to nucleate and grow or, as in the case of intrinsic composites, for crystalline phases to grow and coarsen. The solid state transformation path can be generally influenced by the annealing temperature $\left(T_{a}\right)$ and time $\left(t_{a}\right)$, then the new microstructure can be "frozen-in" by a rapid quench to room temperature where the diffusion process is usually so slow that any reaction can no longer proceed.

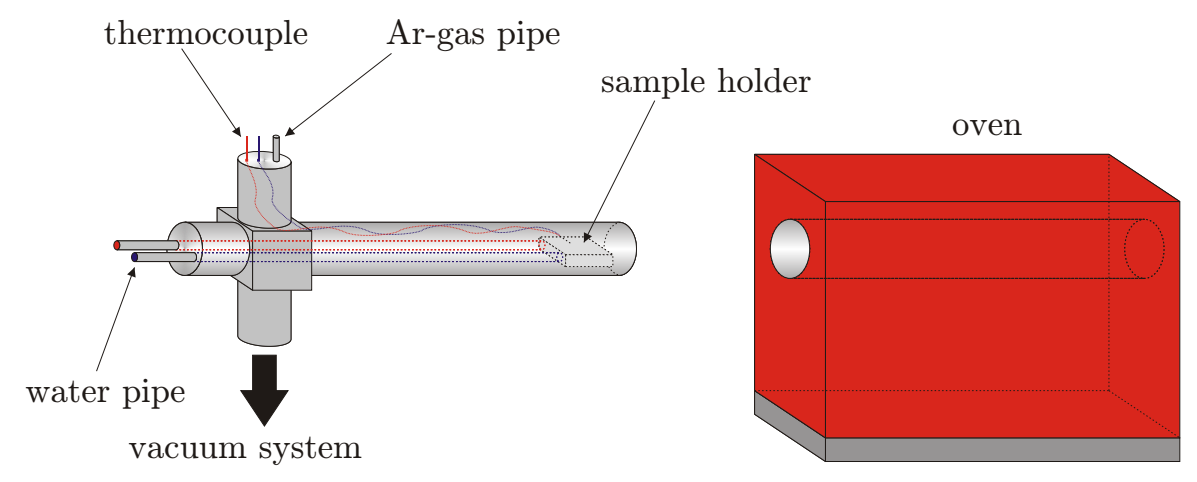

Figure 5.24. Schematic view of the set-up used for the quasi instantaneous annealing and quenching of $\mathrm{Nd}_{60} \mathrm{Fe}_{30} \mathrm{Al}_{10}$ splat-foils and bulk samples. The picture on the right shows the sample older and the thermocouple element which monitored the sample temperature.

The necessity to rapidly heat up a bulk sample to a certain temperature and then to quench it fast requires the use of a special set-up (see figure 5.24). With this solution samples were positioned onto a specimen holder in a cylindrical chamber which can be evacuated or filled with inert gas (Ar). The annealing temperature can be controlled by moving the whole set-up in a temperature gradient produced by a horizontal oven and monitored by a thermocouple fixed to the sample holder and recorded by a computer through a specific program written in Labview ${ }^{\circledR}$. The rapid quench after a certain annealing time is obtained by cooling the holder with a continuous water flux entering the chamber through a pipe.

An example of the sample temperature as function of the annealing time is shown in figure 5.25 as recorded during a thermal treatment and quasi instantaneous quenching of $\mathrm{Nd}_{60} \mathrm{Fe}_{30} \mathrm{Al}_{10}$ samples. 


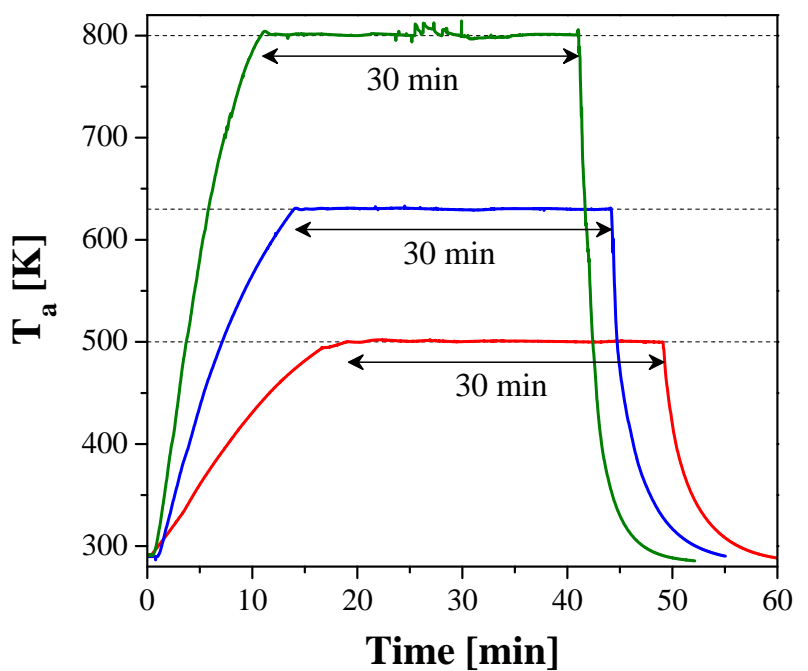

Figure 5.25. Sample temperature as function of time during three annealing treatments at 500,630 , and $800 \mathrm{~K}$, respectively. Both bulk and splat-cooled samples were quickly heated up to the annealing temperature $T_{a}$ and, after $30 \mathrm{~min}$, were rapidly cooled down to room temperature (cooling rate of about $180 \mathrm{~K} / \mathrm{min}$ ). 


\section{Chapter 6}

\section{Results and Discussion}

As anticipated in chapter 1, aim of this work is the structural, thermodynamical, and magnetic characterization of the $\mathrm{Nd}_{60} \mathrm{Fe}_{30} \mathrm{Al}_{10}$ alloy through the preparation and the investigation of four different types of specimens: bulk samples, "master alloy" (see section 2.2.1.2, splat-cooled foils, and thin films. The samples possess the same nominal chemical composition but, due to the different methods which were employed for their preparation (see section 2.2), the samples experienced different cooling rates, ranging from some $\mathrm{K} / \mathrm{s}$ to $10^{10} \mathrm{~K} / \mathrm{s}$, which strongly influence the physical properties. Chapter 6 is dedicated to the presentation and discussion of those properties. The experimental results of the as-prepared $\mathrm{Nd}_{60} \mathrm{Fe}_{30} \mathrm{Al}_{10}$ specimens are presented in the first sections 6.16 .4 , while section 6.5 is dedicated to the annealed samples and, hence, to the evolution of the structural and magnetic properties of the $\mathrm{Nd}_{60} \mathrm{Fe}_{30} \mathrm{Al}_{10}$ alloy upon post-preparation thermal treatments.

\subsection{Bulk Samples}

$\mathrm{Nd}_{60} \mathrm{Fe}_{30} \mathrm{Al}_{10}$ bulk samples have been produced by casting in a cylindrical copper mold of about $3 \mathrm{~mm}$ in diameter (see figure 6.1) with a cooling rate of approximately $10^{2} \mathrm{~K} / \mathrm{s}$

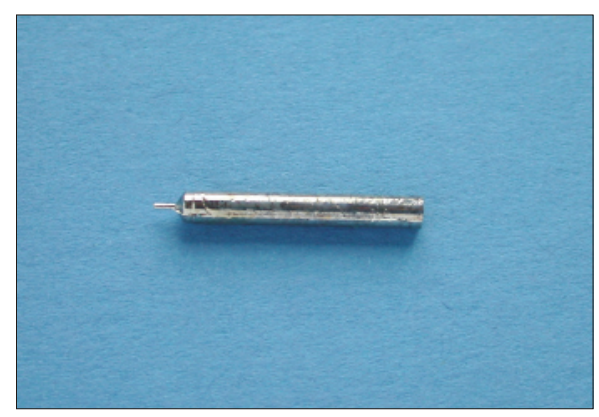

Figure 6.1. Mold cast $\mathrm{Nd}_{60} \mathrm{Fe}_{30} \mathrm{Al}_{10}$ sample cast in a cylindrical mold of $3 \mathrm{~mm}$ in diameter and $20 \mathrm{~mm}$ long. 
(see section 2.2.1.4). For their investigation, the specimens have been cut in $0.5 \mathrm{~mm}$ thick discs and studied by different characterization techniques as $\mathrm{x}$-ray diffraction, small-angle neutron, and scattering to SQUID magnetometry.

\subsubsection{X-Ray Diffraction and Transmission Electron Microscopy}

High-energy wide-angle $\mathrm{x}$-ray scattering (HE-WAXS), high-resolution electron microscopy (HRTEM), and small-angle neutron scattering (SANS and pSANS) have been employed for the structural characterization of the $\mathrm{Nd}_{60} \mathrm{Fe}_{30} \mathrm{Al}_{10}$ samples (the experimental details have been presented in section 5).

The two-dimensional (2-D) HE-WAXS pattern of an as-cast sample measured at $301 \mathrm{~K}$ is presented in Figure 6.2. As the diffraction measurement confirms, specimens

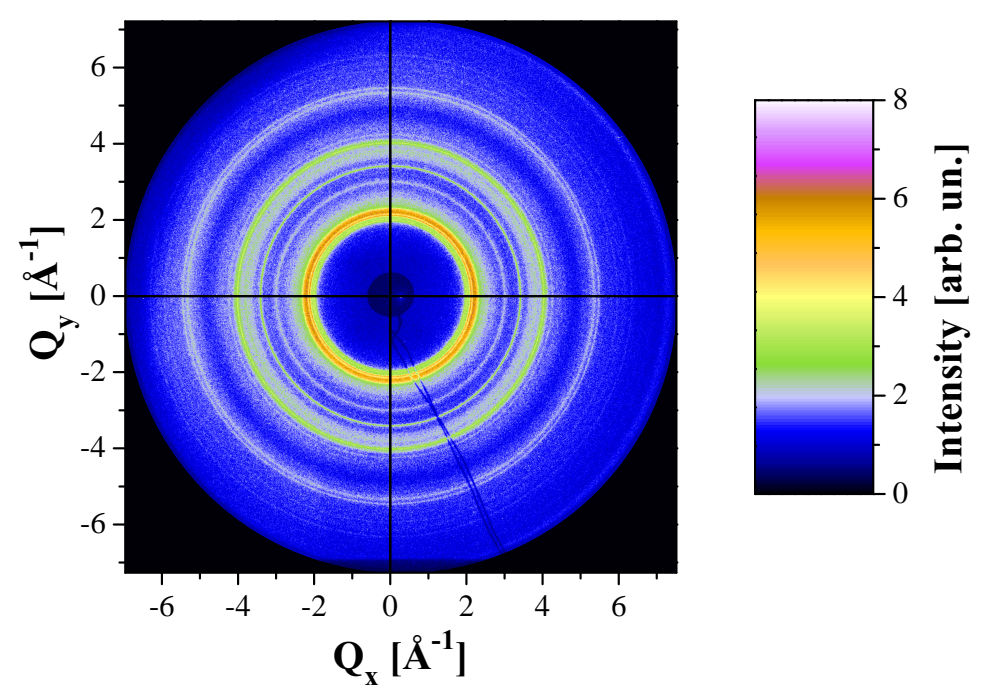

Figure 6.2. Diffuse high-energy wide-angle $x$-ray scattering pattern of a mold-cast $\mathrm{Nd}_{60} \mathrm{Fe}_{30} \mathrm{Al}_{10}$ bulk sample measured at $301 \mathrm{~K}$.

prepared by mold-casting are not fully amorphous but composed of randomly oriented crystals, responsible for the symmetric and sharp Debye rings, and an amorphous matrix, which contributes to the total intensity with broad halos at $Q \simeq 2.2,3.9$ and $5.4 \AA^{-1}$. Due to its isotropic nature, the $2-\mathrm{D}$ pattern of figure 6.2 can be averaged following the reduction method described in section 5.6, and the resultant one-dimensional curve is presented in figure 6.3 (left). The graph expresses the diffraction intensity vs. the scattering vector $Q$ which has been defined in equation 5.75 . The spectrum is characterized by a typical liquid-like contribution due to a glassy phase which overlaps strong reflections originating from a crystalline phase. The strong reflections can be successfully indexed taking into account the crystalline structure of metallic Nd. At room temperature, Neodymium has a double hexagonal closed-packed (dhcp) structure as schematically illustrated in figure 6.4. A dhcp lattice differs from the two other forms of close-packed structures: face centered 

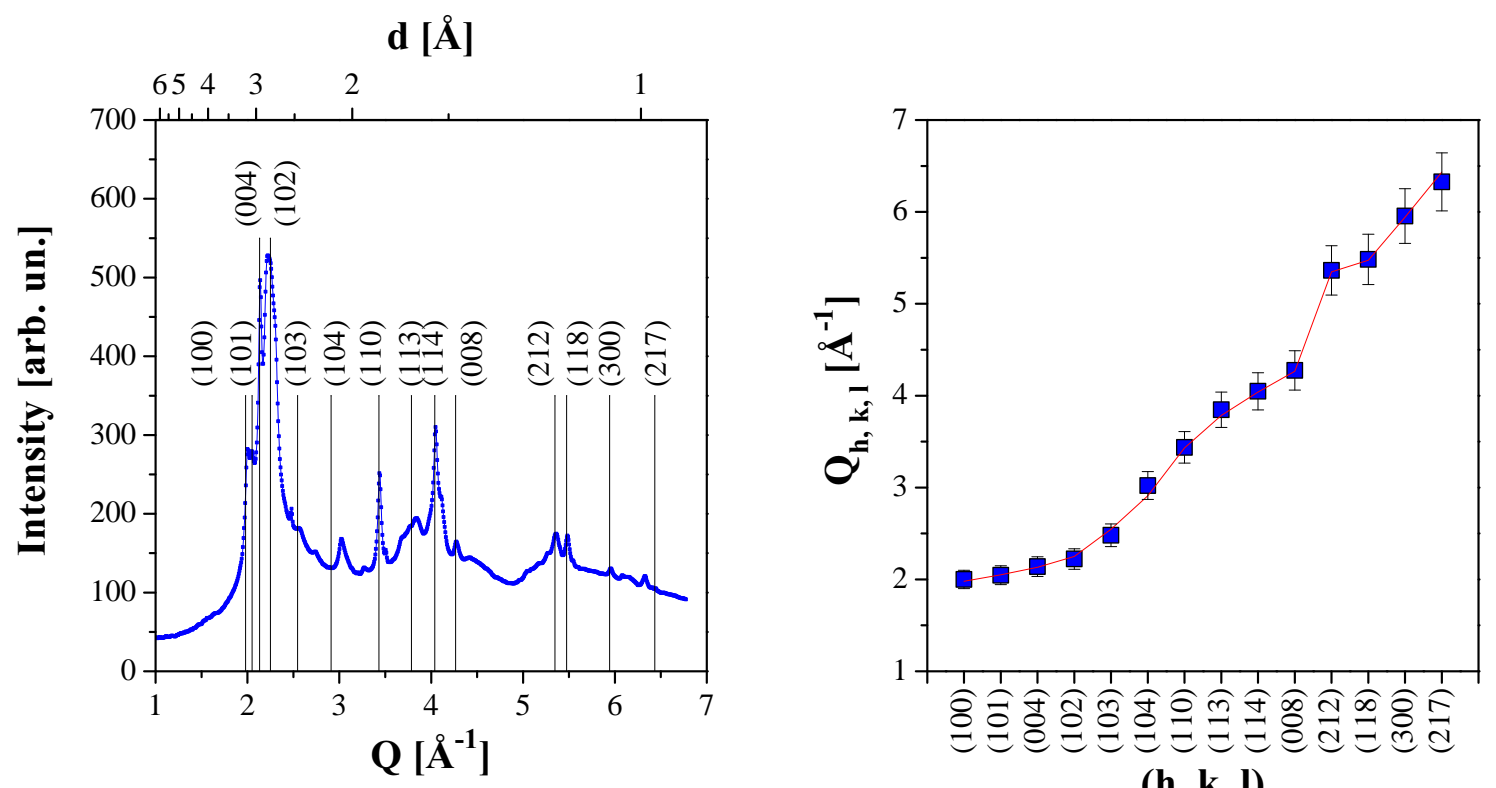

$(h, k, l)$

Figure 6.3. (Left) Diffraction intensity vs. scattering vector $Q$ of a $\mathrm{Nd}_{60} \mathrm{Fe}_{30} \mathrm{Al}_{10}$ bulk sample obtained by azimuthal averaging of the HE-WAXS measurement reported in figure 6.2. (Right) $Q$-values of the diffraction peaks visible in the left graph ( $\boldsymbol{\square})$ and the fit according to equation 5.79 (一).

cubic (fcc) and hexagonal closed-packed (hcp), by its characteristic stapling sequence: ABACA (fcc: ABCA, hcp: ABA). Most of the light rare-earth elements crystallizes in the dhcp lattice 83,84 and several physical properties (i.e., magnetic order and the ordering temperatures, magnetostriction, magnetization, etc.) of those metals are strongly influenced by its atomic arrangement.

The lattice constants of the dhcp structure can be evaluated taking into account the distance between two parallel planes which can be calculated considering the relation for a simple hexagonal Bravais lattice 7

$$
d_{h, k, l}=\frac{a}{\sqrt{\frac{2}{3}\left(h^{2}+k^{2}+j^{2}\right)+l^{2} \cdot\left(\frac{a}{c}\right)^{2}}}
$$

where $j=-h-k, a$ and $c$ are the cell constants of the dhcp structure, and $(h, k, l)$ are the crystallographic indices. According to the von Laue formalism (see equation 5.79, the diffraction condition for the family of planes $(h, k, l)$ is (equation 5.79 of page 100

$$
Q_{h, k, l}=\frac{2 \pi}{d_{h, k, l}}
$$

where $Q_{h, k, l}$ is the resultant value of the scattering vector. Figure 6.3 (right) shows the values of $Q_{h, k, l}$ relative to the diffraction peaks of the WAXS measurement and the fitting according to equation 5.79. A good accordance with the experimental data can be found 

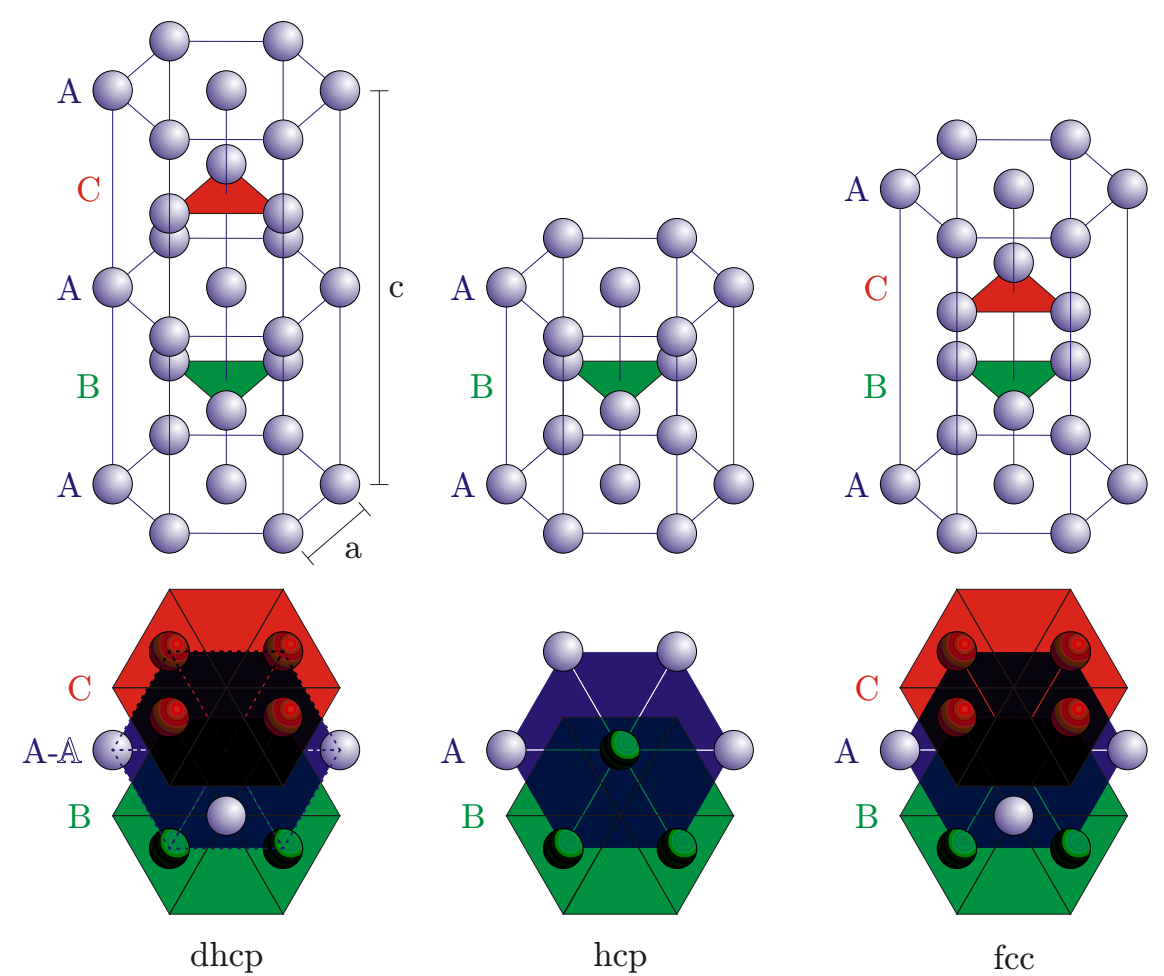

Figure 6.4. Schematic illustration of a double hexagonal closed-packed (dhcp) unit cell (stapling sequence ABACA) characterizing the crystalline structure of most of the light rare-earth elements. The face centered cubic (fcc), and hexagonal closed-packed (hcp) structures are included for comparison.

for $a=(3.663 \pm 0.018) \AA$ and $c=(11.783 \pm 0.101) \AA\left(R^{2}=0.9989\right)$. Those value permit to index all the observed reflections apart of few exceptions and, furthermore they are in good agreement with the lattice constants found for metallic Neodymium $(a=3.6582 \AA$ and $c=11.7970 \AA)|84|$, suggesting that the crystalline phase is likely dhcp Nd-rich with probably only few atomic percentages of $\mathrm{Fe}$ and $\mathrm{Al}$ (the solubilities of $\mathrm{Fe}$ and $\mathrm{Al}$ in $\mathrm{Nd}$ are less than $1 \%$ according to the equilibrium phase diagrams reported in figure 6.5).

Two more information can be gained from the WAXS measurement of figure 6.3 . the mean crystals size and their volume fraction. However, due the complexity of the diffraction pattern, only a rough estimation can be given. The mean size of the $\mathrm{Nd}$ crystals is estimated by applying the Scherrer formula (see equation 5.86 of page 99) to the peak width at half maximum (FWHM) of the (110) reflection. The FWHM value is calculated by fitting the diffraction peak with a Lorentz curve and the resultant crystals mean size is $t=(13 \pm 1) \mathrm{nm}$. The peak and the background areas can then be used to determine the volume fraction $x$ of the nanocrystalline phase. Applying equation 5.91 of page 102, $x$ results to be roughly $15 \%(1)$. The value of $t$ has been confirmed by repeating

\footnotetext{
(1) The composition of the amorphous matrix is assumed to be $\mathrm{Nd}_{60} \mathrm{Fe}_{30} \mathrm{Al}_{10}$ and the crystals are considered as pure Nd. The crystallinity deduced from the diffraction pattern is roughly $36 \%$.
} 

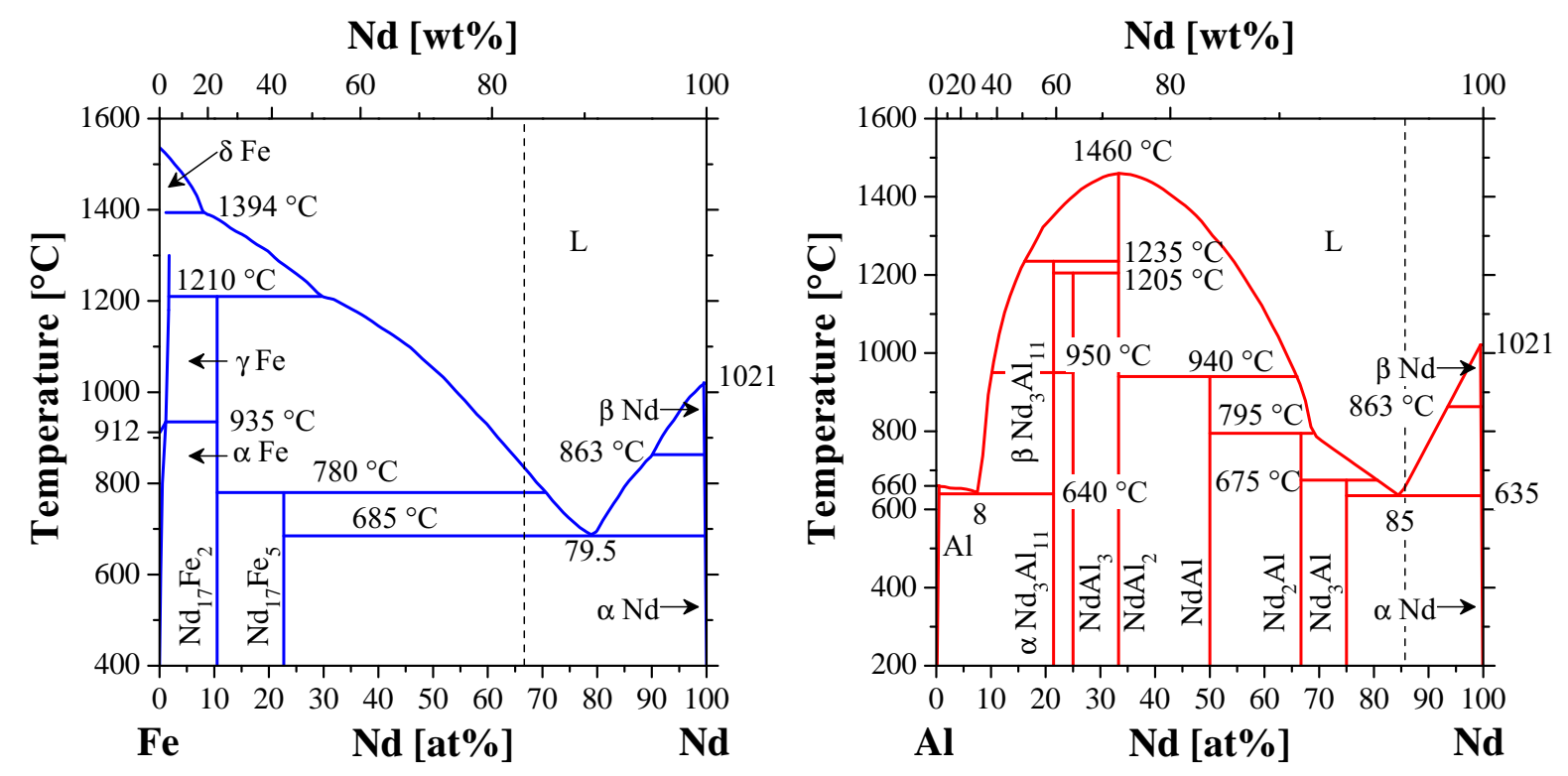

Figure 6.5. Phase diagrams of the binary alloys $\mathrm{Nd}-\mathrm{Fe}$ (left) and $\mathrm{Nd}-\mathrm{Al}$ (right). The dashed lines represent the ratio $\mathrm{Nd}-\mathrm{Fe}$ and $\mathrm{Nd}-\mathrm{Al}$ in the $\mathrm{Nd}_{60} \mathrm{Fe}_{30} \mathrm{Al}_{10}$ alloy.

the same refining method to two other reflections: (004) and (114), and by high-resolution electron microscopy which gives $t=(12 \pm 5) \mathrm{nm}$. Figure 6.6 shows two TEM images of a bulk sample: a low-magnification conical dark-field (see section 5.4) and a highresolution picture of a $\mathrm{Nd}$-rich nanocrystalline grain embedded in an amorphous matrix. As it is clearly seen, nanocrystalline aggregates (bright and dark areas) coexist with an amorphous phase (gray areas). The crystalline phase forms an extended network over the whole sample, but its volume fraction is small compared to that of the glassy phase. Energy-dispersive $\mathrm{x}$-ray microanalysis (EDX) confirms that the nanocrystalline phase is $\mathrm{Nd}$-rich with a Neodymium concentration larger than $80 \%$ ( $\mathrm{Fe}$ and $\mathrm{Al}<10 \%$ ) while the remnant amorphous matrix is close to the nominal composition $\mathrm{Nd}_{60} \mathrm{Fe}_{30} \mathrm{Al}_{10}$. Selectedarea electron diffraction shows a typical pattern for a composite material with crystalline reflexes overlapping liquid-like halos (see figure 6.6). The analysis of the SAD image reveals, however, that the crystalline reflexes can only be indexed taking into account a complex fcc-like structure called bixbyite [177], and not by the expected Nd-dhcp one. The averaged SAD curve and the relative fit according to a bixbyite lattice are shown in figure 6.7. A good accordance can be found for a cell constant $a=(11.43 \pm 0.01) \AA$. The differences between HRTEM and WAXS results can be easily explained taking into account the high tendency of the rare-earth elements to oxidize. In fact, although the samples for the HRTEM characterization have been thinned in an inert Ar atmosphere by cooling them with liquid nitrogen (see section 5.4.1), the $\mathrm{Nd}$-rich nanocrystals oxidize and form $\mathrm{Nd}_{2} \mathrm{O}_{3}$ bixbyite. The cell constant of the $\mathrm{Nd}$-bixbyite reported in the literature is $a=11.048 \AA$ [131, 177], in agreement with the $11.43 \AA$ found in this work. The 

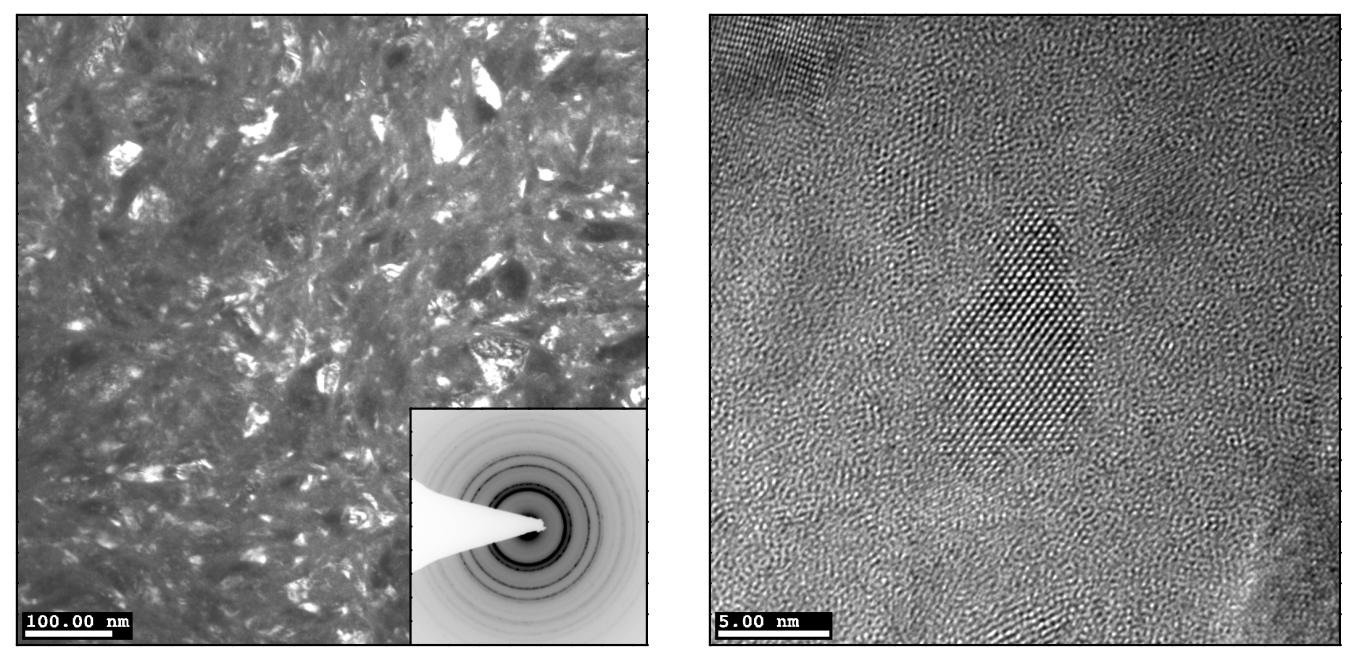

Figure 6.6. (Left) Conical dark field image and the relative selected-area electron diffraction (SAD) picture of a bulk $\mathrm{Nd}_{60} \mathrm{Fe}_{30} \mathrm{Al}_{10}$ sample. The HRTEM results confirm that bulk samples are intrinsic composites in which nanocrystalline agglomerates (bright and dark areas) coexist with a glassy phase (gray areas). (Right) High-resolution image of a Nd-rich nanocrystal.
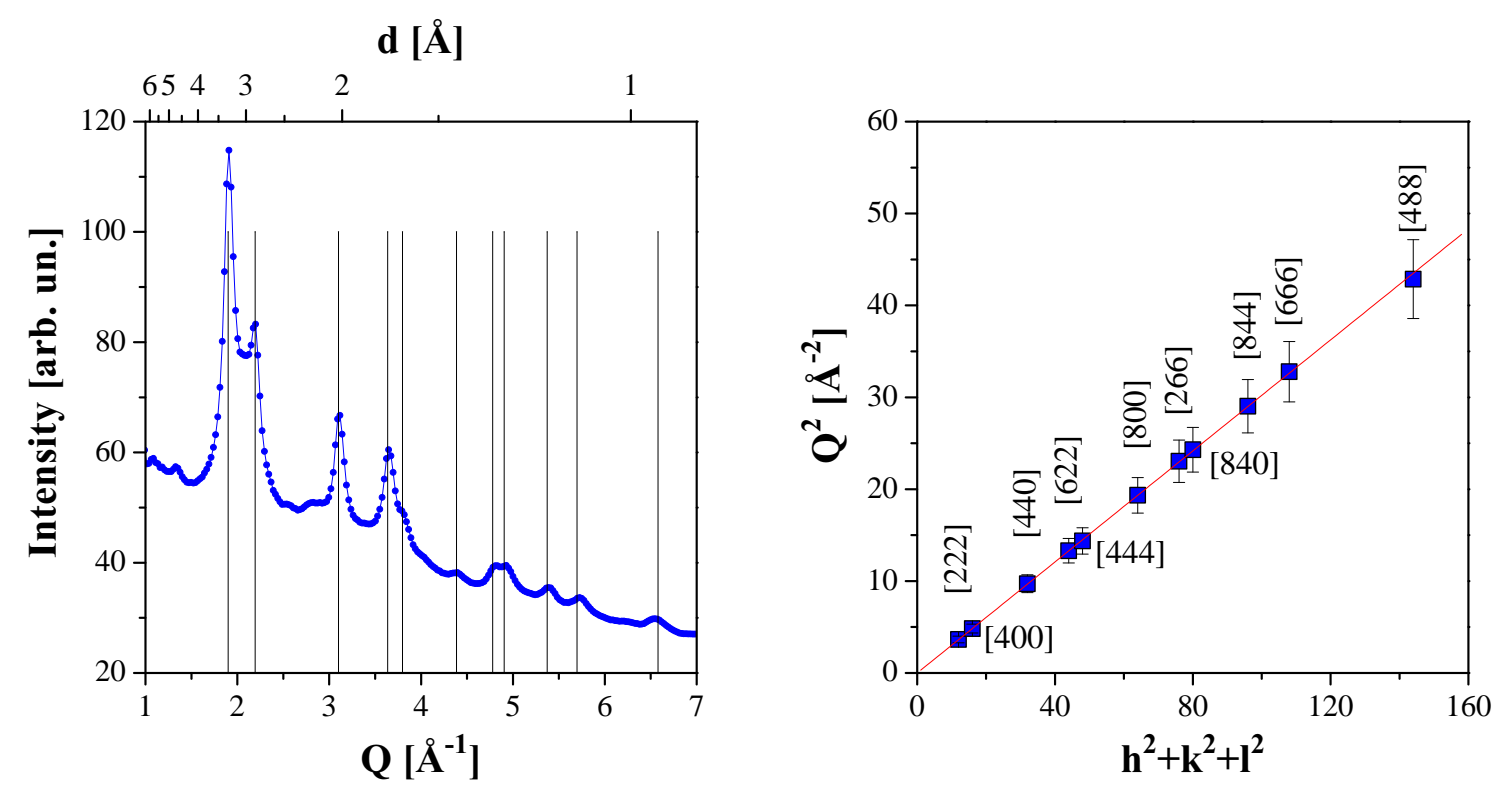

Figure 6.7. (Left) Angular average of the selected-area diffraction pattern presented in the inset of figure 6.6. The diffraction intensity is expressed as function of the scattering vector $Q$. (Right) $Q$-values of the measured diffraction reflections $(\boldsymbol{\square})$ and the relative fitting $(-)$ in accordance to a bixbyite structure with lattice constant $a=(11.43 \pm 0.01) \AA$ $\left(R^{2}=0.9997\right)$. 
observation by HRTEM of Nd nanocrystals with fcc-like structure has been reported by several groups [123, 168, however the presence of bixbyite seems to confirm that Nd-rich phase is that which is mainly present in $\mathrm{Nd}_{60} \mathrm{Fe}_{30} \mathrm{Al}_{10}$ composites, and that the observed cubic structure is just the direct consequence of an oxidation process upon specimens thinning.

The previous results cannot exclude the presence of other crystalline phases beyond the $\mathrm{Nd}$-rich one. However, the reduced number of the $\mathrm{x}$-ray diffraction peaks and, likely, a relatively low volume fraction do not permit their identification either by WAXS or by HRTEM. Furthermore, the magnetic properties of the know equilibrium phases (e.g., magnetic ordering temperature, magnetocrystalline anisotropy, etc. 95, 158,) do not agree with the experimental data presented in this work (see next sections). Other research groups have reported the presence of several different crystalline phases: $\mathrm{Nd}_{5} \mathrm{Fe}_{17}$ [102, $\mathrm{Nd}_{2} \mathrm{Fe}_{17}$ 99,150,161], $\mathrm{NdFe}_{3}$ [128], $\mathrm{NdFe}_{2}$ [136], $\mathrm{Nd}\left(\mathrm{Fe}_{1-x} \mathrm{Al}_{x}\right)_{2}$,49,145, 161 or $\mathrm{Nd}_{6} \mathrm{Fe}_{13-x} \mathrm{Al}_{1+x}$ [97, $\mathrm{A}_{1}$ [153. But the formation of stable or metastable crystalline phases depends strongly on the sample preparation conditions and differences between the experimental results of this work and of other groups cannot be excluded.

\subsubsection{Small-Angle Neutron Scattering}

On the basis of HRTEM results and the complex morphology of the crystalline precipitates, small-angle neutron scattering (SANS) has been employed to further characterize the microstructure of the bulk samples. Due to the difference between the chemical composition of the nanocrystals network and that of the amorphous matrix, a strong nuclear contrast is expected to emerge in the SANS measurements of those samples (see section 5.3. Assuming that the chemical composition of the matrix is close to the nominal $\mathrm{Nd}_{60} \mathrm{Fe}_{30} \mathrm{Al}_{10}$ and the nanocrystals are pure $\mathrm{Nd}$, the neutron length density difference results to be (equation 5.30 of page 79 .

$$
\Delta \eta=\eta_{\text {matrix }}-\eta_{\text {cluster }}=\left(0.6 \cdot \eta_{\mathrm{Nd}}+0.3 \cdot \eta_{\mathrm{Fe}}+0.1 \cdot \eta_{\mathrm{Al}}\right)-\eta_{\mathrm{Nd}}=1.74 \cdot 10^{-6} \AA^{-2}
$$

where $\eta_{\mathrm{Nd}}$ is the neutron length density of $\mathrm{Nd}$ (see table 5.6). Such difference is about $44 \%$ of the value of $\eta_{\text {matrix }}$.

However the nuclear contrast is not the only contribution to the scattering of a heterogeneous magnetic sample since, as already seen in section 5.3.3. magnetic scattering has to also be taken into account.

For this reason, nuclear and magnetic scattering have been separated following the procedure presented in section 5.3.4 and with the use of an electromagnet producing a magnetic field $\mu_{0} H=1 \mathrm{~T}$. The experiment has been carried out at the SAND beamline of the Intense Pulsed Neutron Source (IPNS) (see section 5.3.6). The analysis of the 2-D scattering pattern has been performed for $\boldsymbol{Q} \| \boldsymbol{H}$ and $\boldsymbol{Q} \perp \boldsymbol{H}{ }^{(2)}$ and by a

\footnotetext{
${ }^{(2)}$ To improve the statistics, the analysis of the $2-\mathrm{D}$ scattering pattern has been not made strictly for
} 


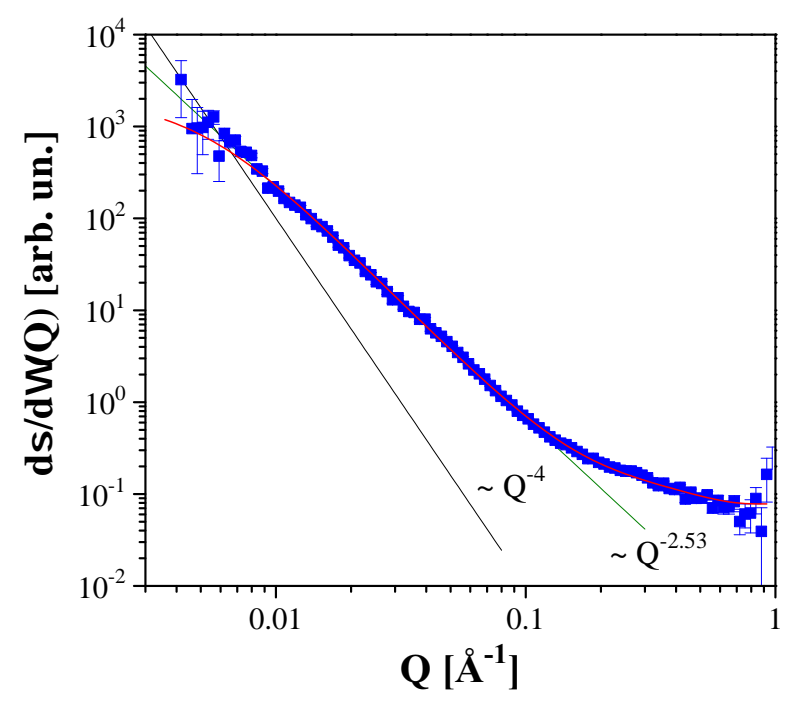

Figure 6.8. SANS cross-section of a $\mathrm{Nd}_{60} \mathrm{Fe}_{30} \mathrm{Al}_{10}$ bulk sample recorded at room temperature ( $\square)$. The curve shows a power law behavior $d \sigma / d \Omega(Q) \propto Q^{2.53}(-)$. The fit is in accordance to the scattering arising from a mass fractal [62 146] (-). A power law $d \sigma / d \Omega(Q) \propto Q^{4}$ is expected for the scattering of non-interacting spheres (-).

$360^{\circ}$ angular averaging over the whole detector. In the $Q$-range from 0.003 to $1 \AA^{-1}$, no significant differences emerge from the sectors analysis and from the comparison of the curves measured with and without magnetic field, suggesting that the magnetic contrasts may be relatively low and that the length scales (e.g., correlation length) are larger than the resolution limit of the SAND which is approximately $100 \mathrm{~nm}$. This results has been confirmed by Wei $[170]$ who reported in $\mathrm{Nd}_{60} \mathrm{Fe}_{20} \mathrm{Co}_{10} \mathrm{Al}_{10}$ observations by magnetic force microscopy indicating domain patterns whose mean size is approximately $360 \mathrm{~nm}$. Thus, it can be concluded that the measured SANS is mainly of nuclear origin. The resultant averaged curve is shown in figure 6.8. The cross section exhibits a power law behavior $Q^{-\alpha}$ in a wide $Q$ region $\left(0.005-0.2 \AA^{-1}\right)$ and a feature at high $Q$-values that corresponds to small objects. The exponent $\alpha$ is about 2.5 which is much smaller than the value arising from scattering spheres of constant size, or with a distribution of radii, for which a $Q^{-4}$-behavior is expected (see the definition of the Porod's regime in section 5.3 .2 and references 62,155]). In this context, Schmidt 137 has shown that aggregate formation through a diffusion limited aggregation (DLA) 176 process leads to a mass fractal object 165] with a power law exponent of 2.5 [120,121,122]. The clustering of small particles into large ramified objects is a commonly occurring phenomena in biological systems, in

$\overline{\boldsymbol{Q} \| \boldsymbol{H}(\alpha=0) \text { and } \boldsymbol{Q} \perp \boldsymbol{H}\left(\alpha=90^{\circ}\right)}$ but by angular averaging in $30^{\circ}$-large sectors $(|\alpha|<15$ and $\left|\alpha-90^{\circ}\right|<15$, respectively). This is still a good approximation since for $H=H_{\max }$ (see section 5.3.4)

$$
\begin{gathered}
\left\langle\sin ^{2}(\alpha)\right\rangle_{ \pm 15^{\circ}} \approx 0 \\
\left\langle\sin ^{2}(\alpha)\right\rangle_{90^{\circ} \pm 15^{\circ}} \approx 1
\end{gathered}
$$


colloid and polymer chemistry, and even in metallurgy. The fundamental parameters in the description of the geometrical arrangement of the particles in a scale-invariant cluster are the particle size $r_{0}$ and the fractal dimension $d_{f}$, which describes how the mass of the cluster increases with its linear dimension $r$

$$
M(r) \propto\left(\frac{r}{r_{0}}\right)^{d_{f}}
$$

and the upper cutoff length $\xi$ which represents the characteristic distances above which the mass distribution in the sample is no longer described by the fractal law of equation 6.3. In practice, $\xi$ can represent the size of an aggregate or a correlation length in a disordered material. Following the formalism proposed by Teixeira [155], the scattering intensity arising from a fractal aggregate embedded in a homogeneous matrix is given by the expression (see section $\mathrm{C}$ and equation C.11)

$$
\frac{d \sigma}{d \Omega}(Q)=B+P \cdot F^{2}\left(Q, r_{0}\right) \cdot\left(\frac{\xi}{r_{0}}\right)^{d_{f}} \cdot \frac{\Gamma\left(d_{f}-1\right)}{Q \xi} \cdot \frac{\sin \left[\left(d_{f}-1\right) \arctan (Q \xi)\right]}{\left[1+(Q \xi)^{2}\right]^{\frac{\left(d_{f}-1\right)}{2}}}
$$

(where $F^{2}\left(Q, r_{0}\right)$ is the form factor for a sphere of radius $r_{0}$, see equation B.2, which reduces to

$$
\frac{d \sigma}{d \Omega}(Q) \propto Q^{-d_{f}}
$$

in the range $\xi^{-1}<Q<r_{0}^{-1}$.

The fitting of the SANS curve according to equation C.11 is presented together with the experimental data in figure 6.8. A good agreement between equation C.11 and the experimental data can be found for the values of the parameters $B, P, r_{0}, d_{f}$, and $\xi$ presented in table $6.1\left(R^{2}=0.993\right)$.

\begin{tabular}{cc}
\hline Parameter & Value \\
\hline$B$ & $0.078 \pm 0.003$ \\
$P$ & $0.07 \pm 0.01$ \\
$r_{0}$ & $(5.2 \pm 0.4) \AA$ \\
$d_{f}$ & $2.53 \pm 0.01$ \\
$\xi$ & $(188 \pm 21) \AA$ \\
\hline
\end{tabular}

Table 6.1. Fit parameters according to equation C.11 and relative to the SANS curve shown in figure 6.8 of a $\mathrm{Nd}_{60} \mathrm{Fe}_{30} \mathrm{Al}_{10}$ bulk sample.

This result has been confirmed by measuring the same sample at $T=460 \mathrm{~K}$, close to its Curie temperature (see section 6.1.5 where the magnetic contribution becomes effectively negligible and only the nuclear contrast contributes to the total scattering intensity. 
Small-angle neutron scattering shows therefore that bulk samples has a complex microstructure composed of a network of atoms aggregates whose mean lateral size is approximately $40 \mathrm{~nm}(2 \xi)$ which is much smaller than the value found by $\mathrm{x}$-ray diffraction $(\approx 13 \mathrm{~nm})$. The different crystallographic orientations of the particles do not permit however to derive $\xi$ from the FWHM of the diffraction peak but the aggregate size clearly emerges from the low-magnification (conical dark-field) TEM image of figure 6.6. On the contrary, the TEM picture is only a projection of a $d_{f}$-dimensional object on a $2-\mathrm{D}$ plane and the mass fractal dimension $d_{f}$ cannot be therefore directly evaluated.

The WAXS, HRTEM and SANS data indicate that the $\mathrm{Nd}_{60} \mathrm{Fe}_{30} \mathrm{Al}_{10}$ bulk samples are intrinsic composites with a complex microstructure. The structural features of this alloy are likely to arise from a phase separation process taking place in the melt or in the undercooled liquid state.

\subsubsection{AC and DC Magnetometry}

In order to characterize the magnetic behavior and to determine the role of the two-phases microstructure, magnetic measurements have been performed as function of temperature and magnetic field in the range between 1.8 and $600 \mathrm{~K}$. If the observed phase separation proceeds towards two or more phases, they may exhibit different magnetic properties. Those properties have been determined by performing $\mathrm{AC}$ and $\mathrm{DC}$ measurements with the use of a SQUID magnetometer (see section 5.1), for $T \leq 400 \mathrm{~K}$, and a VSM (see section 5.2 for $T>300 \mathrm{~K}$.

Figure 6.9 presents the hysteresis loop of $\mathrm{Nd}_{60} \mathrm{Fe}_{30} \mathrm{Al}_{10}$ bulk sample recorded at $300 \mathrm{~K}$ between $\pm 5 \mathrm{~T}$. The bulk sample shows typical hard magnetic behavior with a coercive field of about $250 \mathrm{kA} / \mathrm{m}(\approx 3 \mathrm{kOe})$. The net magnetization at $\mu_{0} H=5 \mathrm{~T}\left(M^{5 T}\right)$ is only $183 \mathrm{kA} / \mathrm{m}$ (see table 6.2 , the reduced remanence $M_{r} / M^{5 T}$ approximately 0.5 and, consequently, the product $\left(\mu_{0} M \cdot H\right)_{\max }$ results to be relatively small compared to other RE-TM systems.

\begin{tabular}{cc}
\hline Parameter & Value \\
\hline$M^{5 \mathrm{~T}}$ & $183 \mathrm{kA} / \mathrm{m}$ \\
$M_{r}$ & $93 \mathrm{kA} / \mathrm{m}$ \\
$H_{c}$ & $246 \mathrm{kA} / \mathrm{m}$ \\
$\left(\mu_{0} M \cdot H\right)_{\max }$ & $19 \mathrm{~kJ} / \mathrm{m}^{3}$ \\
\hline
\end{tabular}

Table 6.2. Values of the magnetization at $5 \mathrm{~T} M^{5 \mathrm{~T}}$, of the remanence $M_{r}$, of the coercivity $H_{c}$, and of the product $\left(\mu_{0} M \cdot H\right)_{\max }$ measured at $300 \mathrm{~K}$ for a $\mathrm{Nd}_{60} \mathrm{Fe}_{30} \mathrm{Al}_{10}$ bulk sample.

Even if the saturated state of the magnetization has been not reached, the curve shows the expected hysteresis of a one-phase system. $\mathrm{Nd}-\mathrm{Fe}-(\mathrm{Co})-\mathrm{Al}$ melt-spun ribbons and 
Field [kOe]

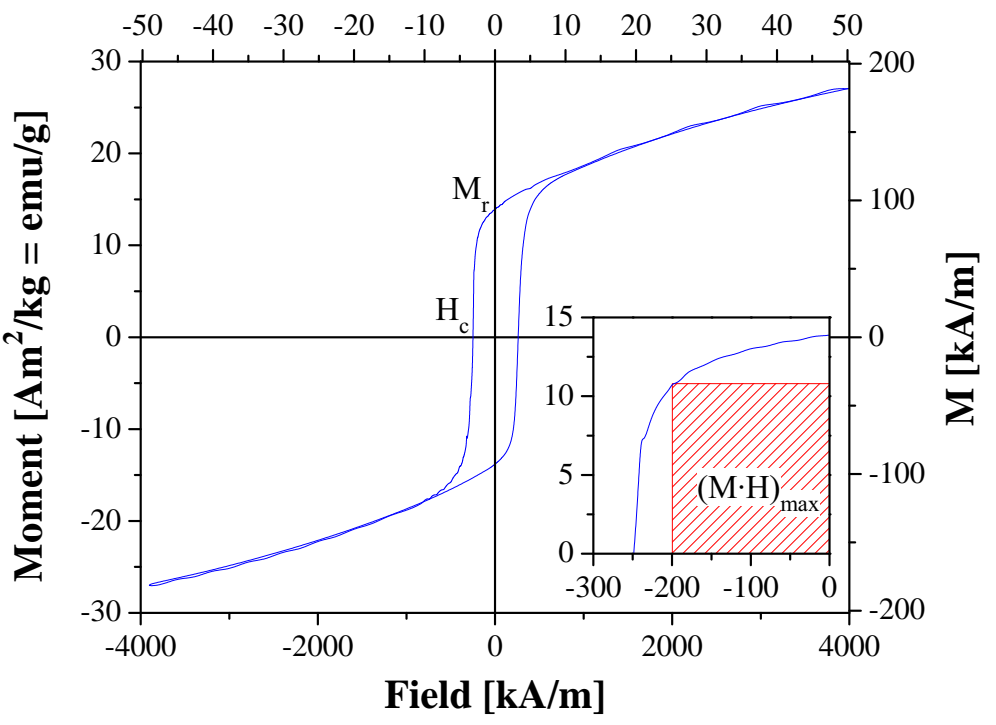

Figure 6.9. Hysteresis loop of a $\mathrm{Nd}_{60} \mathrm{Fe}_{30} \mathrm{Al}_{10}$ bulk sample recorded at $300 \mathrm{~K}$ (一). The red area in the inset expresses the product $(M \cdot H)_{\max }$ which gives a value of $\left(\mu_{0} M \cdot H\right)_{\max }$ equal to $19 \mathrm{~kJ} / \mathrm{m}^{3}$.

$\mathrm{Nd}_{60} \mathrm{Fe}_{30} \mathrm{Al}_{10}$ bulk specimens have proved to have saturation fields larger than $22 \mathrm{~T}$ [161 and $30 \mathrm{~T}$ [128], respectively. The high saturation field is, presumably, due to non-collinear sperimagnetic structure (see section 4.2 of this system and it may be interpreted in terms of the random anisotropy model introduced in section 4.3. However, the role of the $\mathrm{Nd}$ magnetocrystalline anisotropy on the random anisotropy and on the magnetic properties of this alloy must be yet understood.

The evolution of the those properties below and above room temperature has been evaluated by thermomagnetic measurements in the temperature range between 1.8 and $600 \mathrm{~K}$. Figure 6.10 presents the thermomagnetic plot of a bulk sample recorded with a magnetic field of $1 \mathrm{~T}$. For the low-temperature scan, the sample has been measured by applying the external field before cooling to $1.8 \mathrm{~K}$ (field-cooling, or FC). Clearly, two ferromagneticto-paramagnetic transitions appear at about $50 \mathrm{~K}$ and $500 \mathrm{~K}$, in accordance with other results reported for $\mathrm{Nd}_{60} \mathrm{Fe}_{30} \mathrm{Al}_{10}$ bulk specimens and melt-spun ribbons [152,161. Taking into account that the magnetic ordering temperature $T_{C}$ of a $\mathrm{RE}_{1-x} \mathrm{TM}_{x}$ system $(\mathrm{RE}$ $=$ rare-earth, $\mathrm{TM}=$ transition metal) strongly depends on the concentration $x$ of the transition metal [26], the two magnetic transitions indicate the presence of two phases with clearly different chemical compositions and confirm the previous structural results.

In the mean field model [10,74, the ordering temperature of RE-TM amorphous alloys can be expressed by considering the relation

$$
T_{C}=\frac{1}{2}\left(T_{r}+T_{m}+\sqrt{\left(T_{m}-T_{r}\right)^{2}+4 T_{r m}^{2}}\right)
$$

where $T_{r}, T_{m}$, and $T_{r m}$ represent the contributions to $T_{C}$ due to the magnetic coupling 


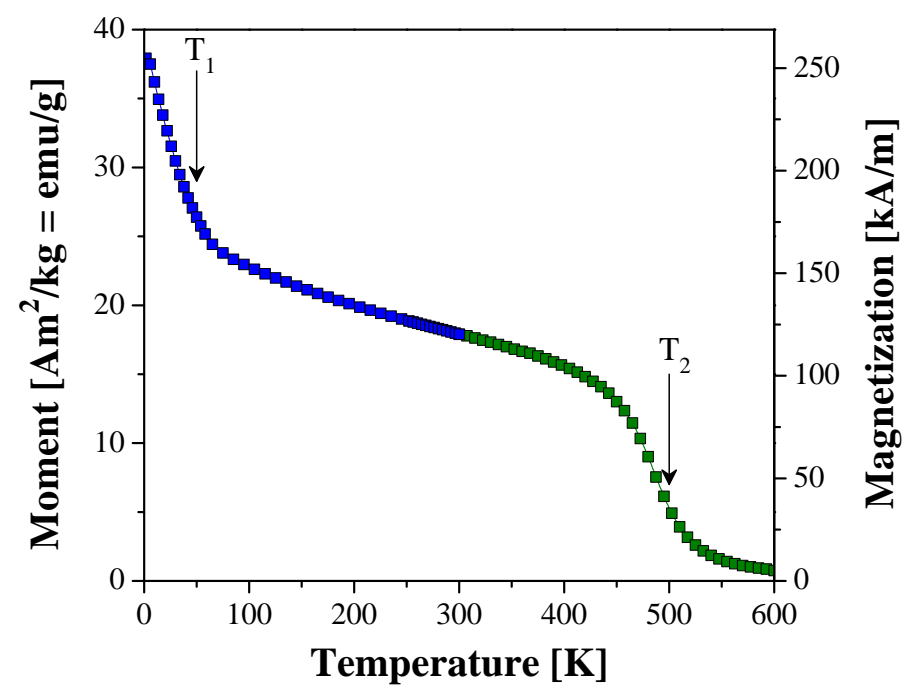

Figure 6.10. Temperature dependence of the magnetic moment and of the magnetization of a field-cooled (1 T) bulk sample measured with a SQUID magnetometer in the range from 1.8 to $300 \mathrm{~K}(\boldsymbol{\square})$, and with a VSM from 300 to $600 \mathrm{~K}(\square) . T_{1}$ and $T_{2}$ indicate the onsets of two ferromagnetic-to-paramagnetic transitions.

between RE-RE, TM-TM, and RE-TM atoms, respectively. If the contribution of $T_{m}$ is considered as that of $\mathrm{Y}_{60} \mathrm{Fe}_{40}$ (Yttrium is in fact a non-magnetic RE element) $\left(T_{C}=15 \mathrm{~K}\right.$ ) and of $T_{r}$ as that of $\mathrm{Nd}_{69} \mathrm{Ni}_{31}|25|\left(T_{C}=18 \mathrm{~K}\right)$, it can be assumed that $\left.T_{r} \simeq T_{m}<T_{r m}\right)$ and the main contribution $T_{C}$ of the $\mathrm{Nd}_{60} \mathrm{Fe}_{30} \mathrm{Al}_{10}$ alloy is from rare-earth and transitionalmetal coupling $T_{r m}$ 151. Therefore the high- $T_{C}$ must be related to a phase whose chemical composition is likely the nominal one. Furthermore this phase is amorphous since, after annealing above a certain temperature, the samples are non-paramagnetic only below $\sim 50 \mathrm{~K}$ (see section 6.5 ). Therefore it must be concluded that the low $-T_{C}$ is a stable crystalline phase.

The effects of the low-temperature transitions on the magnetic properties of the bulk sample can be better estimated by calculating the temperature derivative of the magnetization. Figure 6.11 shows $(d M / d T) / M$ vs. $T$ derived from the thermomagnetic curve reported in figure 6.10. Two minima can be clearly observed: a broad one between 10 and about $35 \mathrm{~K}$, and a second at $500 \mathrm{~K}$, indicating that the low-temperature transition is characterized by a complex magnetic process which affects strongly the magnetization mechanisms of the sample.

These results have been confirmed by AC magnetometry measurements. The temperature dependence of the real $\left(\chi^{\prime}\right)$ and of the imaginary $\left(\chi^{\prime \prime}\right)$ AC susceptibility measured on an as-cast sample is shown in figure 6.12. The $\chi^{\prime}(T)$ and $\chi^{\prime \prime}(T)$ curves present clearly two peaks at $T_{1} \simeq 8 \mathrm{~K}$ and $T_{2} \simeq 33 \mathrm{~K}$, and a strong increase for $T>300 \mathrm{~K}$, revealing the appearance of three different magnetic transitions. $T_{1}$ and $T_{2}$ are close to the magnetic ordering temperatures of $\mathrm{Nd}$ which presents two antiferromagnetic transitions: $T_{N 1}=8.2 \mathrm{~K}$ 


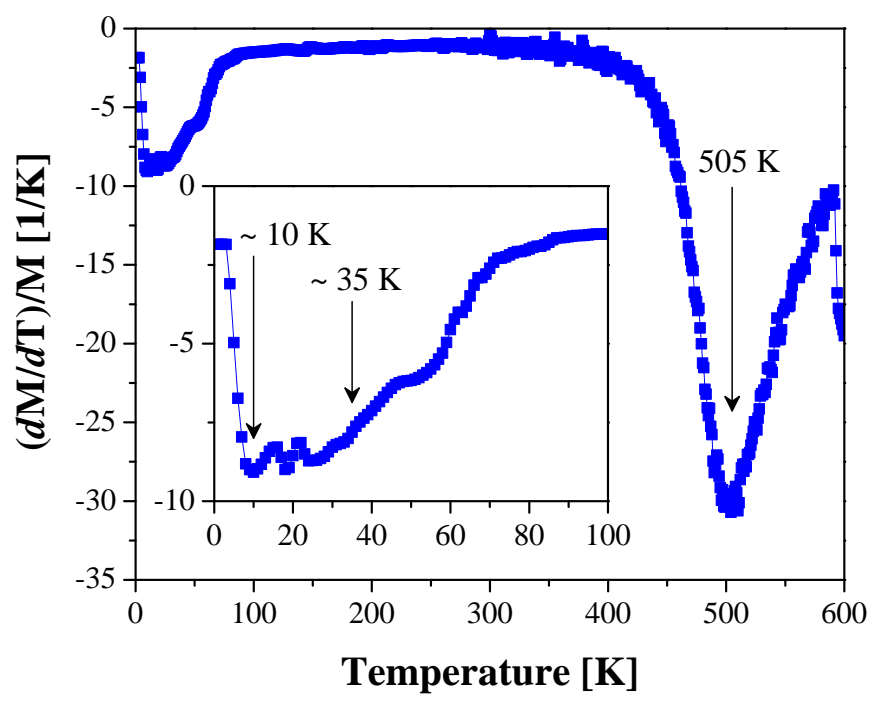

Figure 6.11. Normalized derivative $(d M / d T) / M$ vs. $T$ calculated from the FC thermomagnetic scan shown in figure 6.10 .

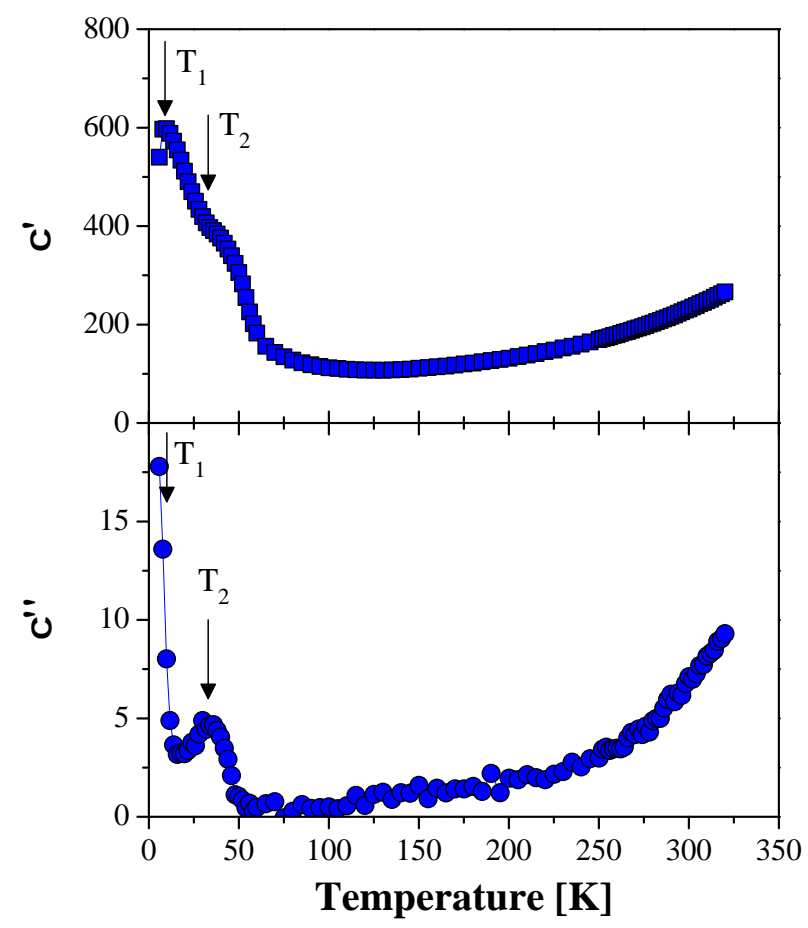

Figure 6.12. Real (top) and imaginary (bottom) AC susceptibility of a bulk as-cast sample measured with a SQUID magnetometer in the temperature range from 5 to $320 \mathrm{~K}$ (driving field: 4 Oe, wave frequency: $117.04 \mathrm{~Hz}$ ). 
(ordering on the $\mathrm{B}$ and $\mathrm{C}$ sites with cubic symmetry, see figure 6.4), and $T_{N 2}=19.9 \mathrm{~K}$ (ordering on the A sites with hexagonal symmetry) 84 p. 57 (3). These results indicate that the low-temperature transitions can be likely attributed to the crystalline Nd-rich phase which is embedded in a high- $T_{C}$ Fe-rich amorphous matrix.

However, from figure 6.10 it is not possible to determine the single contribution of the two transitions to the increase of the magnetization below $50 \mathrm{~K}$ and, hence, it cannot be distinguished if the two transitions are both related to ferromagnetic ordering, to antiferromagnetism or even to a complex metamagnetic transition due to inequivalent local surrounding.

The low-temperature behavior of the bulk samples has been further investigated by measuring the temperature dependence of the DC magnetization as function of the magnetic field. For this scope, the samples have been cooled down to $1.8 \mathrm{~K}$ with (FC) and without applying an external magnetic field (zero-field cooling, or ZFC) ${ }^{(4)}$ and, successively, the magnetic moment has been measured up to $300 \mathrm{~K}$. Different field strengths have been applied during the cooling and the re-heating. Figure 6.13 shows, for example, the FC and ZFC curves relative to fields of 0.5 and $5 \mathrm{~T}$. As indicated by the large

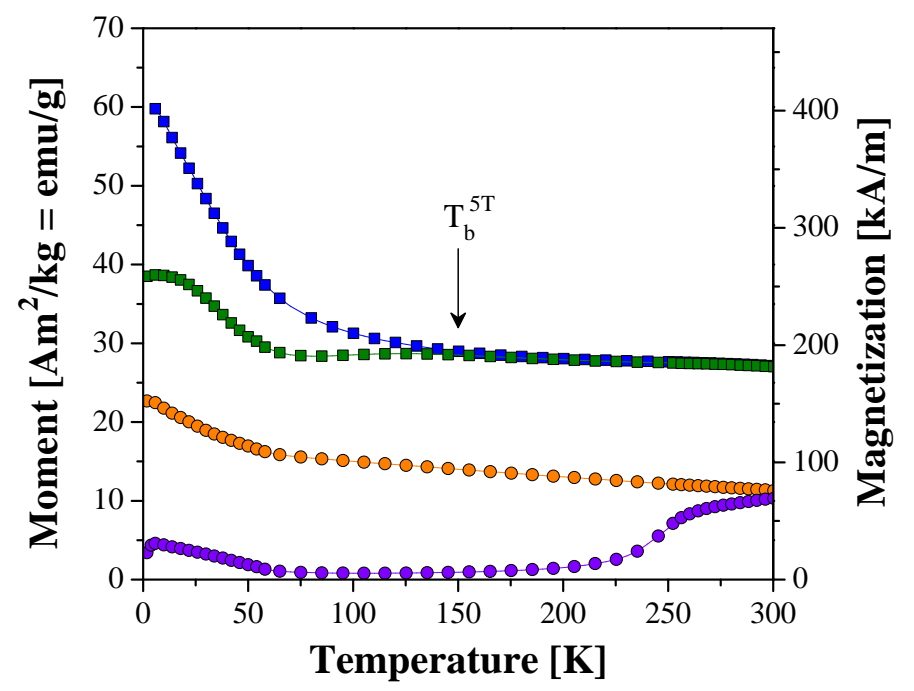

Figure 6.13. Temperature dependence of the field-cooled (FC) and zero-field-cooled (ZFC) magnetization of an as-cast bulk sample measured in the range from 1.8 to $300 \mathrm{~K}$ by applying

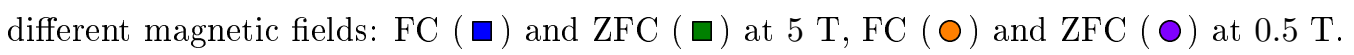

difference between FC and ZFC curves, the magnetization depends strongly on the thermomagnetic method which is used to cool down the sample. With increasing temperature the difference decreases and vanishes completely at a temperature $T_{b}$ which is a function

\footnotetext{
${ }^{(3)} \mathrm{A}$ fcc allotrope of $\mathrm{Nd}$ has been proved to order ferromagnetically at $T_{C}=29 \mathrm{~K}[103$ and it may contribute to the transition at $T \sim 33 \mathrm{~K}$, however fcc-Nd has been proved to form only under high pressure.
}

${ }^{(4)}$ Prior to minor loops demagnetization at room temperature. 
of the applied field. This behavior has been already observed in many different magnetic systems, such as spin-glasses-like [59] $\mathrm{RE}_{69} \mathrm{Fe}_{31}\left[26\right.$ or $\mathrm{RE}_{69} \mathrm{Co}_{31}[24$ amorphous alloys or in crystalline materials such as $\operatorname{Sm}\left(\mathrm{Co}_{(1-x)} \mathrm{Ni}_{x}\right)_{5}[23]$, and it is commonly called thermomagnetic history effect. Numerous magnetic mechanisms may be responsible for it but, generally, the origin of these effects is ascribed to a thermally activated magnetization process which gives rise to a strong temperature dependence of the coercivity. In high-anisotropy systems and speromagnets (see section 4.2), the coercivity is expected to increase continuously with decreasing temperature. When $H_{c}$ is close to, or larger than the applied field, the magnetization of the sample is hampered and low, or zero, $M_{Z F C}$ values are measured. The same behavior can also be observed in systems with pinningtype magnetization (see section 4.4). In this case, thermomagnetic history effects can be understood taking into account thermal activated pinning mechanisms of the magnetic domain-walls. Pinning centers can be defects, impurities or, simply, a second phase which pins the moments of the main magnetic phase hampering their rotation. Those processes are characterized by energy barriers which must be overcome by applying an external magnetic field of a certain strength to achieve the complete unpinning (see section 4.5.1). Thus, at a certain temperature and applied field, only a part of the full magnetization of the zero-field-cooled sample can be aligned, explaining the gap between FC and ZFC. As the temperature is raised, thermal fluctuations begin to reduce the height of the energy barriers increasing the number of magnetic moments which can be unpinned by the external field and, hence, increasing the ZFC magnetization. As soon as the thermal energy $\left(\approx k_{B} T\right)$ is comparable to the height of the energy barriers, the pinning effects are completely overcome and the ZFC curve overlaps the FC one.

In order to clarify the thermo-history effect, which suggests a strong temperature dependence of the coercivity, several hysteresis loops at various temperatures between 1.8 and $300 \mathrm{~K}$ have been measured. Figure 6.14 shows the curves measured at 300, 200, 120, 40, 20, 5, and $1.8 \mathrm{~K}(5)$ respectively. As the temperature progressively decreases, the coercive field increases while the magnetization $M^{5 \mathrm{~T}}$ remains almost constant. Below $50 \mathrm{~K}$, the Nd-rich crystalline phase becomes ferromagnetic as the strong increase of $M^{5 \mathrm{~T}}$ shows, and the coercivity clearly decreases. The hysteresis loops show an evolution of their shape from rectangular for $T>50 \mathrm{~K}$, to a typical two-phases like $(5 \mathrm{~K}<T<50 \mathrm{~K})$ and, finally, to sigmoidal for $T<5 \mathrm{~K}$, demonstrating the complex magnetic nature of the magnetic ordering processes in the bulk samples.

In the temperature range where only the amorphous phase is ferromagnetic, an indication of the mechanism determining the variation of the coercivity may be deduced from the virgin curves of the magnetization (see section 4.4). Figure 6.15 shows the virgin curve measured at $120 \mathrm{~K}$ after demagnetization of the sample at room temperature by mi-

\footnotetext{
${ }^{(5)}$ It is worth to mention that the hysteresis loops measured below $100 \mathrm{~K}$ have been recorded by cooling the samples in a magnetic field of $5 \mathrm{~T}$.
} 


\section{Field [kOe]}

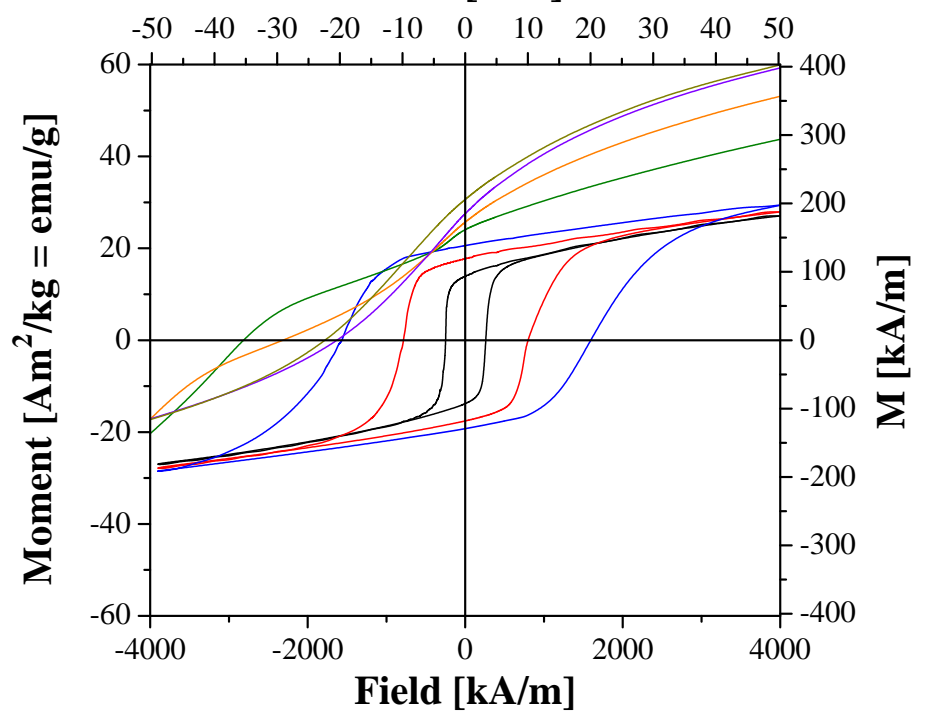

Figure 6.14. Hysteresis loops of a $\mathrm{Nd}_{60} \mathrm{Fe}_{30} \mathrm{Al}_{10}$ bulk sample measured with a SQUID magnetometer at $300(-), 200(-)$ and $120 \mathrm{~K}(-)$. Half hysteresis loops have measured at $40(-), 20(-), 5(-)$, and $1.8 \mathrm{~K}(-)$ after cooling in a $5 \mathrm{~T}$ field. Note the evolution of the loops shape as the sample temperature decreases.

nor loops and zero-field cooling. The small increase of the magnetization at low applied

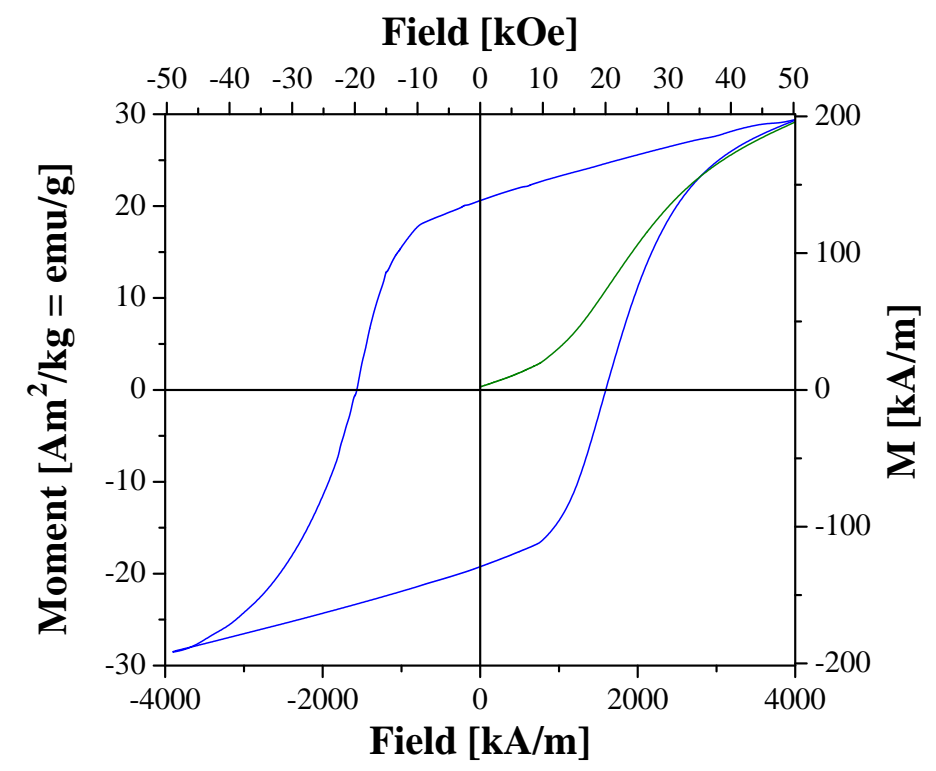

Figure 6.15. Virgin curve (-) and hysteresis loop (-) of a $\mathrm{Nd}_{60} \mathrm{Fe}_{30} \mathrm{Al}_{10}$ bulk sample measured at $120 \mathrm{~K}$. Note the typical pinning-type magnetization (see section 4.4).

fields is a first indication that pinning processes play the major role in those samples. This result is therefore of fundamental importance for the interpretation of the coercivity mechanisms to which the following section 6.1 .4 is dedicated. 


\subsubsection{Coercivity Mechanisms}

$\mathrm{Nd}_{60} \mathrm{Fe}_{30} \mathrm{Al}_{10}$ bulk samples show hard magnetic properties with a typical pinning-like behavior above about $40 \mathrm{~K}$. Domain wall pinning effects must be therefore taken into account as candidates to understand the hard magnetic properties of these samples and, in this context, the role which the complex morphology of the Nd-rich nanocrystals may play as pinning sites, must be determined. For this scope the temperature dependence of the coercive field has been determined by measuring several hysteresis loops between 1.8 and $600 \mathrm{~K}$. The curve $H_{c}$ vs. $T$ is shown in figure 6.16 . The value of $246 \mathrm{kA} / \mathrm{m}$ at room temperature increases rapidly as the temperature decreases, presenting a maximum of $2830 \mathrm{kA} / \mathrm{m}$ at $40 \mathrm{~K}$. Below $40 \mathrm{~K}$, the coercive field decreases steeply and increases again for $T<10 \mathrm{~K}$. The two ordering temperatures found for the Nd-rich phase below

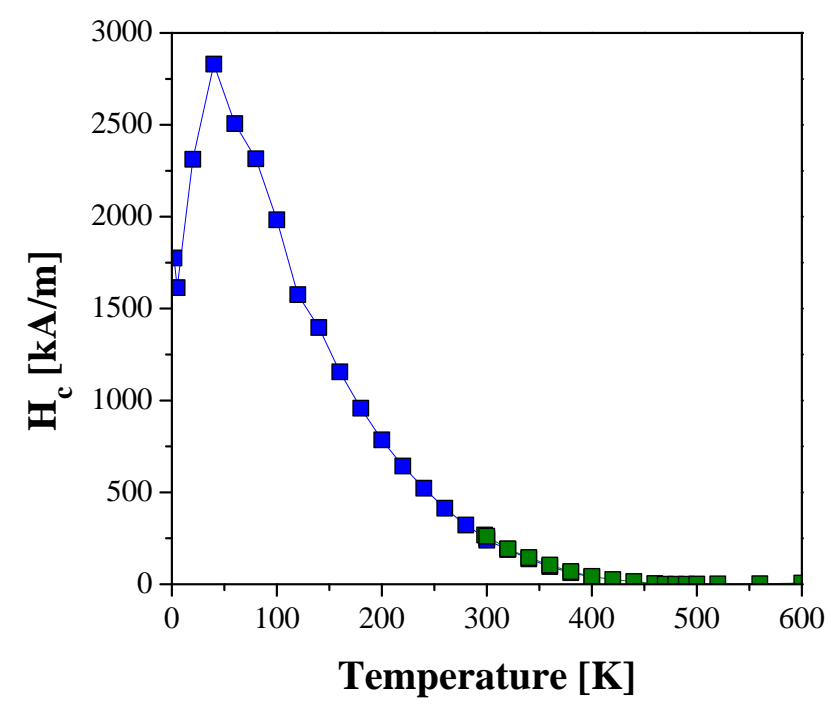

Figure 6.16. Temperature dependence of the coercivity $H_{c}$ extracted from the hysteresis curves in $\mathrm{Nd}_{60} \mathrm{Fe}_{30} \mathrm{Al}_{10}$ bulk samples which have been measured between 1.8 and $400 \mathrm{~K}$ with a SQUID magnetometer (๑), and from 300 to $600 \mathrm{~K}$ with a VSM ( $)$ ).

$35 \mathrm{~K}$ seem to influence strongly the coercivity of this alloy and confirm clearly the interplay between amorphous and crystalline phases. According to the virgin magnetization presented in figure 6.15 and to the curve of figure 6.16, it must be assumed that such interplay arises mainly as pinning of the domain walls in the temperature range where the Nd-rich phase is paramagnetic.

In order to determine the characteristic aspects of the pinning process, the Gaunt formalism introduced in section 4.5.1 has been considered. Gaunt described the temperature dependence of the coercivity in a heterogeneous system characterized by strong pinning through the expression (equation 4.53 of page 61 )

$$
\left(\frac{H_{c}}{H_{0}}\right)^{1 / 2}=1-\left(\frac{T}{T_{0}}\right)^{2 / 3}
$$


considering thermal-activated processes of low-dense pinning centers.

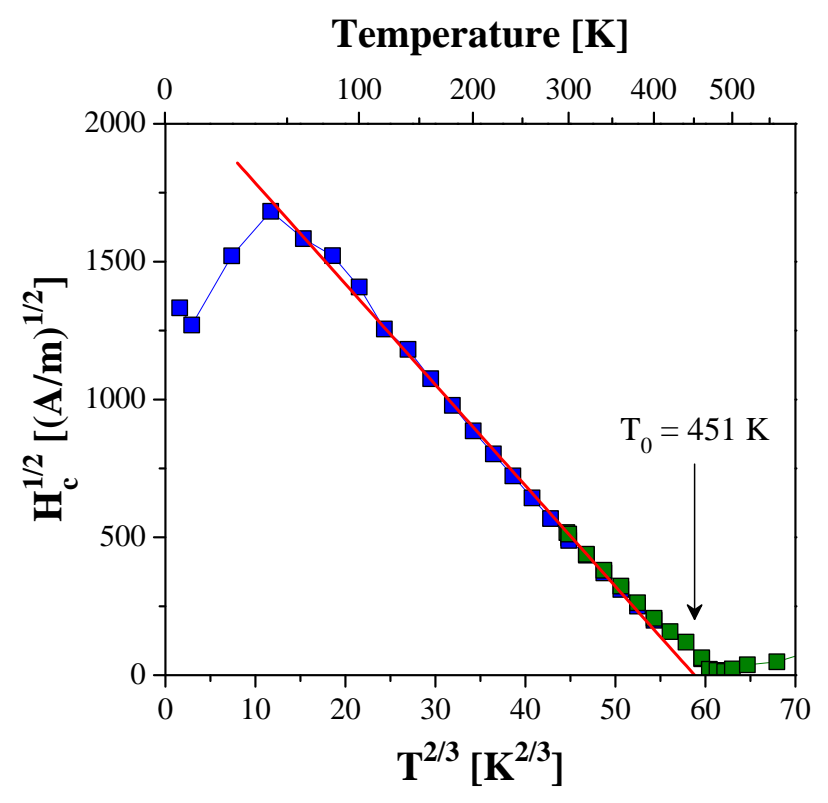

Figure 6.17. Gaunt plot $H_{c}^{1 / 2}$ vs. $T^{2 / 3}$ of the experimental data shown in figure 6.16 The line (-) represent the fit between 40 and $450 \mathrm{~K}$ according to the domain-wall pinning model [63]. The characteristic crossover temperatures $T_{0}$ is indicated by the arrow.

Figure 6.17 shows the plot $H_{c}^{1 / 2}$ vs. $T^{2 / 3}$ relative to the experimental data of figure 6.16 . The Gaunt model is in good agreement with the temperature variation of the coercivity in a large temperature range between 40 and $400 \mathrm{~K}$ and the linear fit permits to determine the two unknown parameters $H_{0}$ and $T_{0}$ which characterize the pinning mechanism and whose values are listed in table 6.3. The coercive field $H_{0}$ indicates that at $T=0 \mathrm{~K}$

\begin{tabular}{cc}
\hline Parameter & Value \\
\hline$H_{0}$ & $(4625 \pm 67) \mathrm{kA} / \mathrm{m}$ \\
$T_{0}$ & $(451 \pm 12) \mathrm{K}$ \\
\hline
\end{tabular}

Table 6.3. Intrinsic coercive field $H_{0}$ and the crossover temperature $T_{0}$ as obtained by fitting the experimental data $H_{c}^{1 / 2}$ vs. $T^{2 / 3}$ of figure 6.17 according to equation 4.53

$H_{c}$ exceeds $4.6 \mathrm{MA} / \mathrm{m}(\approx 57.8 \mathrm{kOe})$ because of strong pinning effects and it decreases rapidly until it vanishes at $T=451 \mathrm{~K}$. However, $H_{c}^{451 \mathrm{~K}}$ cannot be experimentally zero since the domain wall pinning is unlikely the only coercivity mechanism present in the $\mathrm{Nd}_{60} \mathrm{Fe}_{30} \mathrm{Al}_{10}$ bulk samples, although the good agreement between experimental data and the Gaunt model. In fact, in terms of the random anisotropy model (see section 4.3), the amorphous $\mathrm{Nd}-\mathrm{Fe}-\mathrm{Al}$ matrix has an intrinsic anisotropy which determines consequently an intrinsic coercive field. Therefore a deviation from the Gaunt behavior is expected at $T \simeq T_{0}$ since the thermal energy $\left(k_{B} T_{0}\right)$ is comparable to the energy barrier defined by 
the strength of the pinning centers, and other coercivity mechanisms become observable. Such behavior is confirmed experimentally by the slight deviation of the data from the straight line, that can be effectively observed in figure 6.17 for $T \geq 400 \mathrm{~K}$. Nevertheless, the almost perfect linear behavior of $H_{c}^{1 / 2}$ vs. $T^{2 / 3}$ for $T<400 \mathrm{~K}$ clearly demonstrates that strong-pinning centers are responsible for the hard magnetism of the bulk samples.

The Gaunt model allows to estimate density and mean size of the pinning centers through equation 4.41 if the pinning is assumed to be strong. This condition is fulfilled if

$$
\frac{3 f}{2 \pi \gamma_{w} 4 b} \gg 1
$$

where $f$ is the force per pin, $\gamma_{w}$ is the domain wall energy density and $4 b$ a characteristic interaction length. This expression can be rewritten taking into account the definitions of $f$ (see equation 4.52, $\gamma_{w}$, and $4 b[13$ as

$$
\begin{gathered}
f=\frac{75 k_{B} T_{0}}{\delta_{w}} \\
4 b \simeq \delta_{w}=\pi \sqrt{\frac{A}{K}} \\
\gamma_{w}=2 \pi \sqrt{A K}
\end{gathered}
$$

( $A$ and $K$ are the magnetic stiffness and anisotropy constant, respectively), and permits to obtain the following condition for strong pinning

$$
\frac{A^{3 / 2}}{K^{1 / 2}} \ll \frac{225 k_{B} T_{0}}{4 \pi^{4}}=3.6 \cdot 10^{-21} \mathrm{~J}
$$

which can be fulfilled, for example, assuming $A^{3 / 2} / K^{1 / 2}=1 \cdot 10^{-22} \mathrm{~J}$. Then, according to equation 4.38 , the density of pinning sites becomes

$$
\begin{aligned}
\rho=4 \pi \gamma_{w} \frac{\mu_{0} M_{s} H_{0}}{3 f^{2}} & =4 \pi \gamma_{w} \delta_{w}^{2} \cdot \frac{\mu_{0} M_{s} H_{0}}{3 \cdot\left(75 k_{B} T_{0}\right)^{2}}= \\
& =8 \pi^{4} \frac{A^{3 / 2}}{K^{1 / 2}} \cdot \frac{\mu_{0} M_{s} H_{0}}{3 \cdot\left(75 k_{B} T_{0}\right)^{2}} \simeq 1.27 \cdot 10^{23} \mathrm{~m}^{-3}
\end{aligned}
$$

where $M_{s} \simeq M^{5 \mathrm{~T}}=183 \mathrm{kA} / \mathrm{m}$ (see table 6.2 , , or similarly

$$
\rho=\frac{x}{\left(\frac{4}{3} \pi r^{3}\right)}
$$

where $x$ is their volume fraction $(\approx 15 \%$ according to the $\mathrm{x}$-ray data) and $r$ their mean radius. Thus, the strong pinning observed for the $\mathrm{Nd}_{60} \mathrm{Fe}_{30} \mathrm{Al}_{10}$ bulk samples are likely due to pinning centers whose radius is approximately

$$
r=\left(\frac{3 x}{4 \pi \rho}\right)^{1 / 3} \simeq 7 \mathrm{~nm}
$$


This value is much smaller than the mean radius of the Nd-rich nanocrystals $(\xi=$ $19 \mathrm{~nm}$ obtained by SANS) to which pinning effects have been attributed.

In order to give an explanation to this difference, polarized neutrons small-angle scattering (pSANS) has been employed complementary to conventional SANS. Due to the high sensibility to magnetization fluctuations at surfaces and interfaces in heterogeneous structures (see section 5.3.5, pSANS is a fundamental technique in the investigation of $\mathrm{Nd}_{60} \mathrm{Fe}_{30} \mathrm{Al}_{10}$ samples. The pSANS experiments have been performed at the beamline NG-3-30 of the Center for High Resolution Neutron Scattering (CHRNS) (see section 5.3.6. The samples have been measured at room temperature and in a constant magnetic field $\mu_{0} H=1.2 \mathrm{~T}$. In this way large domains (i.e., magnetic correlated volumes) are formed and the only magnetic contribution to small angle scattering remains the contrast between the paramagnetic (fractal-) nanocrystals and the ferromagnetic amorphous matrix. Thus, the paramagnetic clusters can be considered as holes in a ferromagnetic homogeneous environment.

Figure 6.18 shows the pSANS signals for the polarization states $I^{+}$and $I^{-}$, and the relative sum $\left(I^{-}+I^{+}\right)$and difference signals $\left(I^{-}-I^{+}\right)$(see section 5.3.5). As expected, the
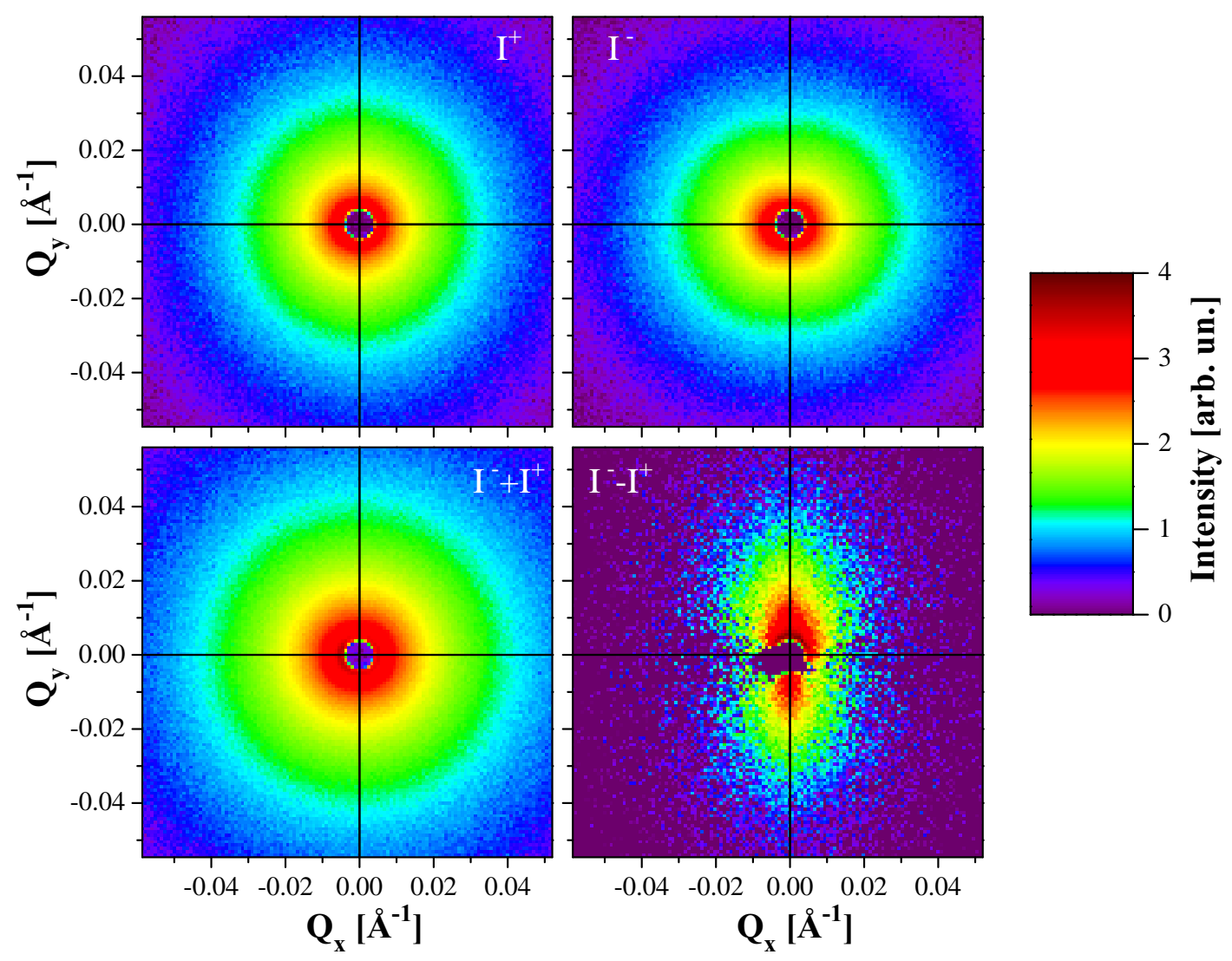

Figure 6.18. Small angle neutrons scattering patterns for the two polarization states $I^{+}$ and $I^{-}$, and for the relative sum $\left(I^{-}+I^{+}\right)$and difference signals $\left(I^{-}-I^{+}\right)$.

sum signal coincides with the 2-D pattern obtained with non-polarized neutrons while the 
difference signal shows the $\sin ^{2}(\alpha)$ behavior expected from equation 5.61 and a negligible intensity along the direction of the magnetic field ( $\mathrm{x}$-axis).

Applying to the 2-D patterns $\left(I^{-}+I^{+}\right)$and $\left(I^{-}-I^{+}\right)$the analysis and the angular averaging presented in section 5.3.5, the nuclear and the magnetic cross sections shown in figure 6.19 have been obtained as function of the scattering vector $Q$. As expected, the
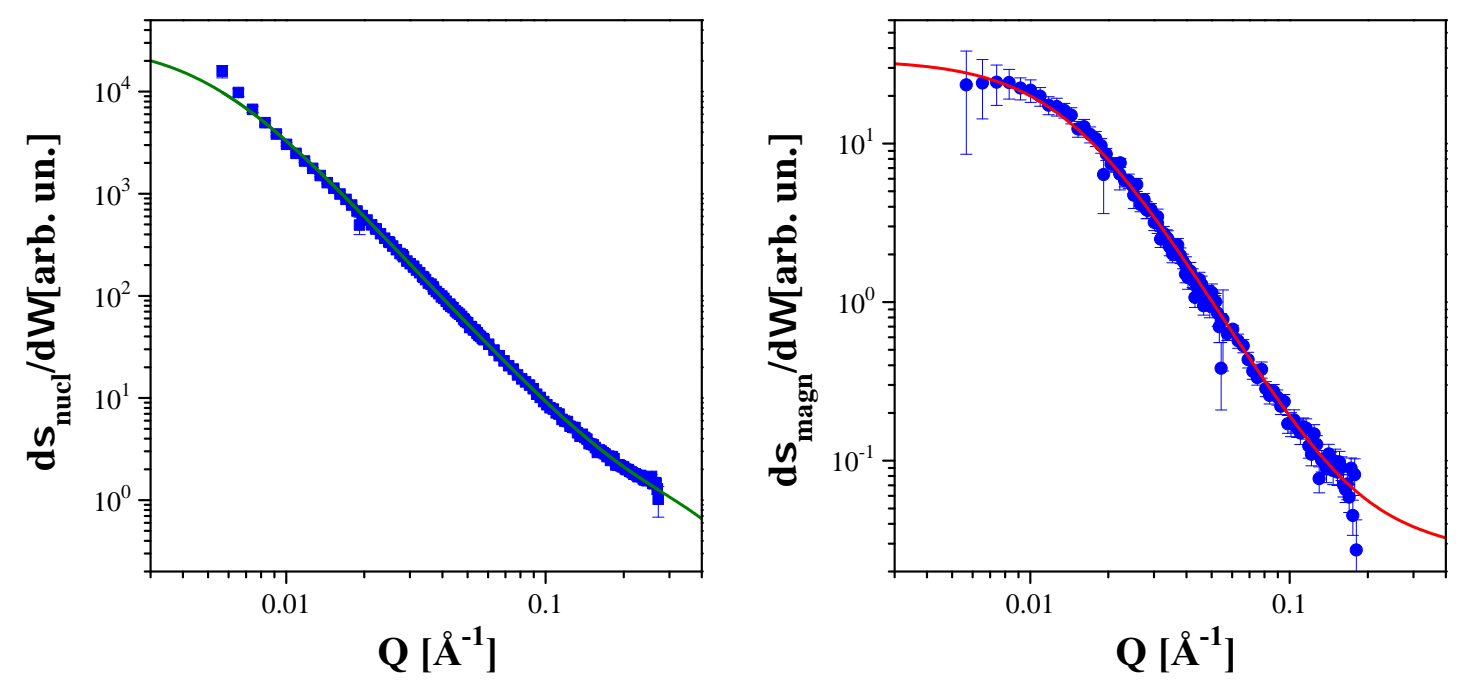

Figure 6.19. Reconstructed nuclear (left) and magnetic (right) cross sections obtained by averaging the 2-D pSANS patterns $\left(I^{-}+I^{+}\right)$and $\left(I^{-}-I^{+}\right)$according to the analysis proposed in section 5.3.5. The line $(-)$ is the fit of the SANS curve of figure 6.8 according to equation C.11 and the parameters listed in table 6.1. The line $(-)$ represents instead the fit of the pSANS magnetic cross sections according to equation C.11 and the parameters listed in table 6.4 .

nuclear signal agrees perfectly with the previous SANS results as the good accordance between the pSANS curve and the fit obtained by equation C.11 with the parameters of table 6.1 clearly shows. The magnetic signal, instead, could not be determined by SANS. The low intensity of the magnetic cross section in figure 6.19 indicates a small contrast between the crystalline clusters and the embedding matrix. Nevertheless such contrast permits to obtain the cluster radius $\xi$ arising from magnetic scattering through the fit of the experimental data and to compare this value with that found by nuclear scattering. A good agreement between experimental data and equation C.11 can be found for the values of the parameters $B, P, r_{0}, d_{f}$, and $\xi$ listed in table $6.4\left(R^{2}=0.985\right)$. The value $\xi$ is in good agreement with the previous calculation on the effective pin radius $(6 \mathrm{~nm})$, based exclusively on the Gaunt analysis of the $H_{c}^{1 / 2}$ vs. $T^{2 / 3}$ curve, and indicates that $\mathrm{Nd}$-rich nanocrystals, whose radius has been estimated by SANS to be approximately $\xi_{\text {nucl }} \simeq 19 \mathrm{~nm}$, have probably a complex core-shell structure composed of a paramagnetic "nucleus" of radius $\xi_{\text {magn }} \simeq 7 \mathrm{~nm}$ and a magnetic shell of thickness $\left(\xi_{\text {nucl }}-\xi_{\text {magn }}\right) \simeq 12 \mathrm{~nm}$.

The different nuclear and magnetic lengths scales explain therefore the observed SANS and pSANS curves, and a further indication of the existence of a core-shell structure arises 


\begin{tabular}{cc}
\hline Parameter & Value \\
\hline$B$ & $0.025 \pm 0.006$ \\
$P$ & $0.0089 \pm 0.0008$ \\
$r_{0}$ & $(4.0 \pm 0.4) \AA$ \\
$d_{f}$ & $2.51 \pm 0.04$ \\
$\xi$ & $(65 \pm 4) \AA$ \\
\hline
\end{tabular}

Table 6.4. Parameters of equation C.11 obtained by fitting the magnetic pSANS cross section shown in figure 6.19 (right).

from the plot of the $Q$-vector dependence of the ratio $\gamma$ (equation 5.66 of page 90 which is shown in figure 6.20. The ratio between magnetic and nuclear cross sections is roughly

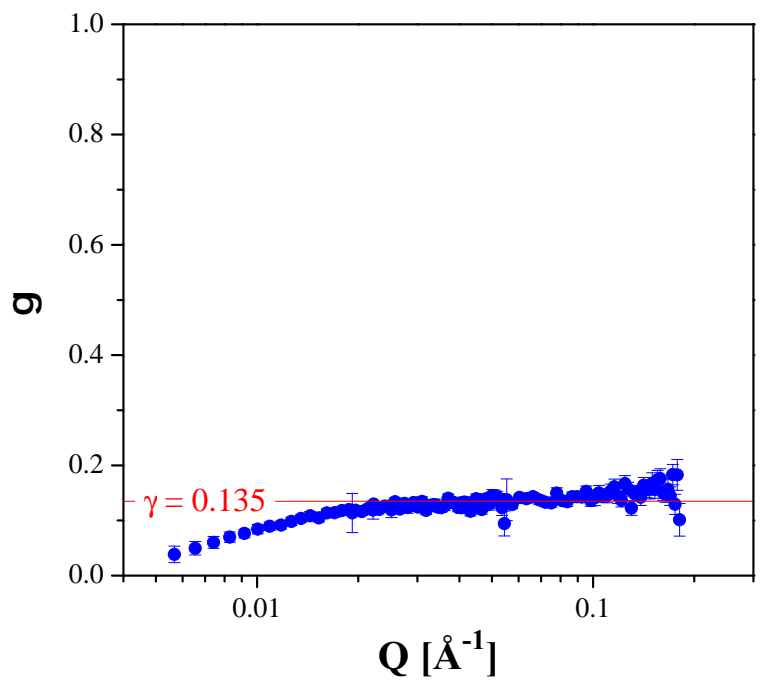

Figure 6.20. Ratio $\gamma$ between magnetic and nuclear cross sections as defined in equation 5.66 .

constant $(\approx 13.5 \%)$ for high $\mathrm{Q}$-values (small length scale) and decreases strongly for $Q<0.03 \AA^{-1}$ (length scale $\approx 20 \mathrm{~nm}$ ). It must be assumed therefore that the complex nanocrystals-amorphous matrix interface is characterized by spatial variation of the chemical composition and of the magnetic properties which can be responsible for the pinning effects observed in those specimens.

\subsubsection{Curie Temperature and Critical Behavior}

In the interpretation of the magnetic behavior of a ferromagnetic system, the most valuable parameter is certainly its critical temperature $T_{C}$ (Curie temperature). At $T$ close to $T_{C}$, the spontaneous magnetization $M_{s}$ and the initial susceptibility $\chi_{0}$ vary asymptoti- 
cally following the relations 13,59

$$
\lim _{H \rightarrow 0} M=M_{0}=a \cdot\left(T_{C}-T\right)^{\beta}
$$

for $T<T_{C}$, and

$$
\lim _{H \rightarrow 0} \frac{H}{M}=\chi_{0}^{-1}=b \cdot\left(T-T_{C}\right)^{\gamma}
$$

for $T>T_{C}$, where $\beta$ and $\gamma$ are commonly called critical exponents. The values of those exponents can be experimentally measured and their comparison with the theoretical predictions may give some hints to the interpretation of the magnetic behavior of the $\mathrm{Nd}_{60} \mathrm{Fe}_{30} \mathrm{Al}_{10}$ system. The critical exponents for three different models are listed in table 6.5 .

\begin{tabular}{cccc}
\hline Model & $\beta$ & $\gamma$ & $\nu$ \\
\hline Mean field & 0.5 & 1 & 0.66 \\
3D Ising & 0.32 & 1.24 & 0.63 \\
3D Heisenberg & 0.36 & 1.39 & 0.71 \\
\hline
\end{tabular}

Table 6.5. Critical exponents of the magnetization $(\beta)$, the susceptibility $(\gamma)$, and of the coherent length $(\nu)$ as predicted by mean field, 3D Ising, and 3D Heisenberg models, respectively. The value of $\nu$ can be calculated from $\beta$ and $\gamma$ by the scaling laws of the equilibrium critical phenomena 59 .

Experimentally, however, the requirement of an external field to measure a magnetic moment and the presence of magnetic domains do not permit to calculate directly $M_{0}$. The values of $M_{0}$ and $\chi_{0}^{-1}$ must be therefore obtained by extrapolation for $H \rightarrow 0$ with the use of the Arrott plots of $M^{1 / \beta}$ vs. $(H / M)^{1 / \gamma}$ [1, 6]. For this reason, several temperature scans have been measured in the range from 300 to $600 \mathrm{~K}$ and for different magnetic fields. $M^{1 / \beta}$ vs. $(H / M)^{1 / \gamma}$ (see for example figure 6.21) can be then plotted for each temperature $T_{i}$ assuming two arbitrary values of $\beta$ and $\gamma \cdot 90,124,178$. From the slope and intercept of the relative linear fits, the curves $M_{0}(T)$ and $\chi_{0}^{-1}(T)$ can be then easily calculated. The critical temperature $T_{C}$ can be obtained from plots of $\left[d \ln \left(M_{0}\right) / d T\right]^{-1}$ vs. $T$ and $\left[d \ln \left(\chi_{0}^{-1}\right) / d T\right]^{-1}$ vs. $T$, since

$$
\begin{gathered}
\frac{d \ln \left(M_{0}\right)}{d T}=\frac{d}{d T}\left[\beta \ln a+\beta \ln \left(T_{C}-T\right)\right]=-\frac{\beta}{T_{C}-T} \\
\frac{d \ln \left(\chi_{0}^{-1}\right)}{d T}=\frac{d}{d T}\left[\gamma \ln b+\gamma \ln \left(T-T_{C}\right)\right]=\frac{\gamma}{T-T_{C}}
\end{gathered}
$$

while the critical exponents $\beta$ and $\gamma$ can be determined through $T_{C}$ by plotting $\log \left(M_{0}\right)$ 


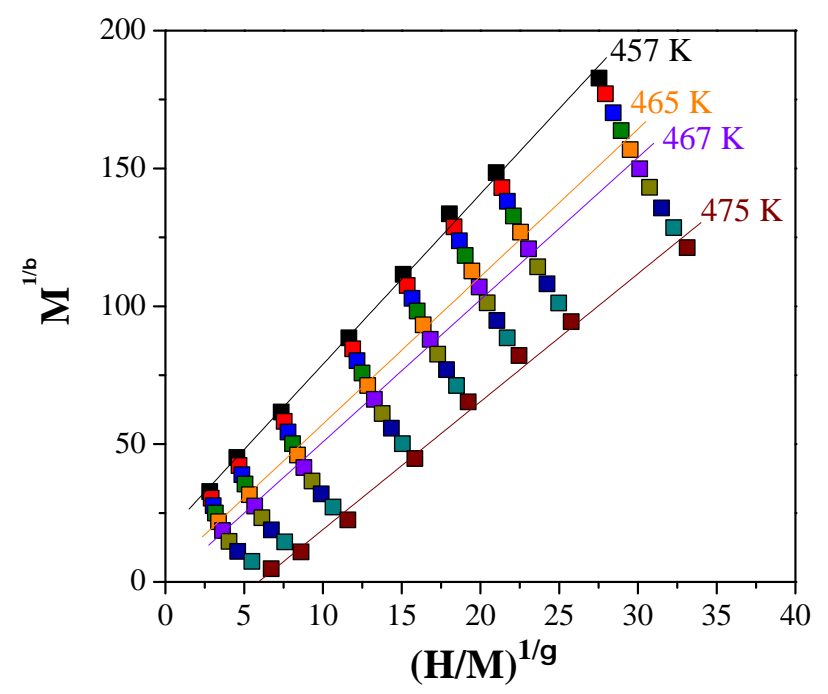

Figure 6.21. Arrott plot $M^{1 / \beta}$ vs. $(H / M)^{1 / \gamma}$ for a $\mathrm{Nd}_{60} \mathrm{Fe}_{30} \mathrm{Al}_{10}$ bulk sample for different temperatures between $457(\boldsymbol{\square})$ and $475 \mathrm{~K}(\boldsymbol{\square})(\beta=0.50, \gamma=1.11)$. The lines represent the relative linear regressions.

vs. $\log \left(1-T / T_{C}\right)$ and $\log \left(\chi_{0}^{-1}\right)$ vs. $\log \left(T / T_{C}-1\right)$, since

$$
\begin{aligned}
& \log \left(M_{0}\right)=c+\beta \cdot \log \left(1-\frac{T}{T_{C}}\right) \\
& \log \left(\chi_{0}^{-1}\right)=d+\gamma \cdot \log \left(\frac{T}{T_{C}}-1\right)
\end{aligned}
$$

where $c$ and $d$ are constants.

The values of $\beta$ and $\gamma$ are, however, not known a priori and they must be determined by iterations. In each iteration, the initial $\beta$ and $\gamma$ in the plot $M^{1 / \beta}$ vs. $(H / M)^{1 / \gamma}\left(\beta_{i}\right.$ and $\gamma_{i}$ ) result in slightly different final values $\beta_{f}$ and $\gamma_{f}$ arising from the plots of $\log \left(M_{0}\right)$ vs. $\log \left(1-T / T_{C}\right)$ and $\log \left(\chi_{0}^{-1}\right)$ vs. $\log \left(T / T_{C}-1\right)$, and, hence, the iteration continues until the differences $\left(\beta_{f}-\beta_{i}\right)$ and $\left(\gamma_{f}-\gamma_{i}\right)$ are negligible.

The iteration has been automatized by implementing a $\mathrm{C}++$ program in the analysis software OriginPro@ (OriginLab Corporation) which carries out the different calculation and fitting processes, and gives out the final values of $T_{C}, \beta$, and $\gamma$.

Figure 6.21 shows the $M^{1 / \beta}$ vs. $(H / M)^{1 / \gamma}$ plot for a $\mathrm{Nd}_{60} \mathrm{Fe}_{30} \mathrm{Al}_{10}$ bulk sample at constant temperatures near $T_{C}$. As shown in figure 6.22 , the plots of $\left[d \ln \left(M_{0}\right) / d T\right]^{-1}$ vs. $T$ and $\left[d \ln \left(\chi_{0}^{-1}\right) / d T\right]^{-1}$ (right) vs. $T$ are as expected straight lines near $T_{C}$. The linear fits give, respectively, $T_{C 1}=471 \mathrm{~K}$ and $T_{C 2}=465 \mathrm{~K}$ and, hence, a mean critical temperature $T_{C}=(468 \pm 4) \mathrm{K}$. The critical exponents result to be $\beta=(0.50 \pm 0.02)$ and $\gamma=(1.11 \pm 0.05)$. Using the scaling relation $3 \nu=2 \beta+\gamma[59$, the value of the critical exponent for the correlation length results to be $\nu=(0.70 \pm 0.05)$.

On the basis of this analysis, the temperature dependence of the spontaneous magnetization $M_{0}(T)$ and of the inverse initial susceptibility $\chi_{0}^{-1}(T)$ can be calculated taking 


\section{Temperature [K]}
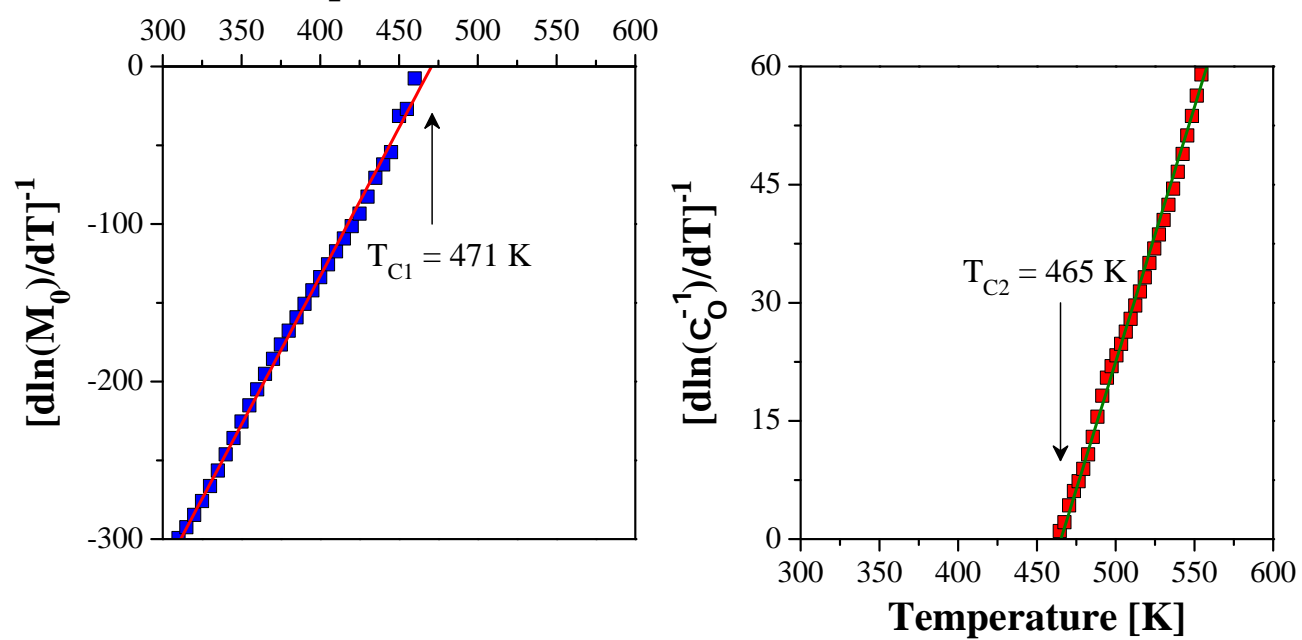

Figure 6.22. Plots of $\left[d \ln \left(M_{0}\right) / d T\right]^{-1}$ (left) and $\left[d \ln \left(\chi_{0}^{-1}\right) / d T\right]^{-1}$ (right) vs. $T$ for a $\mathrm{Nd}_{60} \mathrm{Fe}_{30} \mathrm{Al}_{10}$ bulk sample for the determination of $T_{C}=(468 \pm 4) \mathrm{K}$.

into account equation 6.14 and 6.15 , respectively. Figure 6.23 shows the curves $M_{0}, \chi_{0}^{-1}$

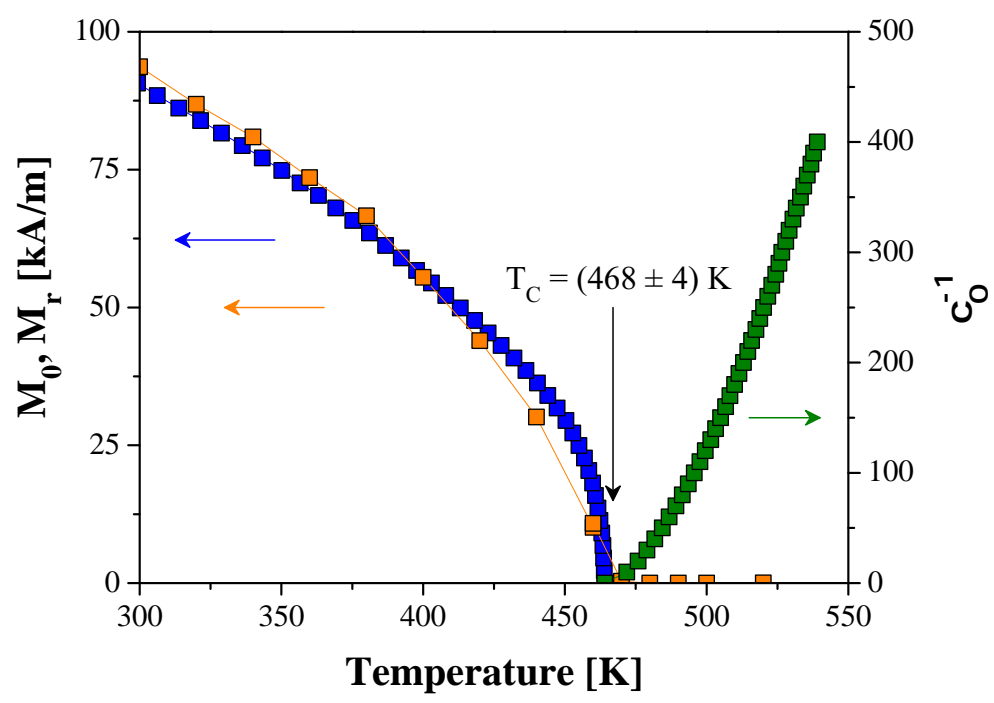

Figure 6.23. Spontaneous magnetization $M_{0}(\boldsymbol{\square})$ and the inverse initial susceptibility $\chi_{0}^{-1}$ ( $\square)$ as deduced from the Arrott analysis for $T_{C}=(468 \pm 4) \mathrm{K}, \beta=(0.50 \pm 0.02)$ and $\gamma=$ $(1.11 \pm 0.05)$. For comparison, the remanence $M_{r}(T)$ deduced from the high-temperature hysteresis curves $(\square)$.

and, for comparison, the remanence $M_{r}(T)$, deduced from the hysteresis curves measured between 300 and $600 \mathrm{~K}$.

The results of the Arrott analysis on the $\mathrm{Nd}_{60} \mathrm{Fe}_{30} \mathrm{Al}_{10}$ bulk specimens are for clarity listed in table 6.6. The critical exponents $\beta$ and $\gamma$ suggest that the magnetic behavior of these samples can be described in term of the mean field model, and, although the ordering temperature of amorphous and composite materials is usually broadened by chemical and 


\begin{tabular}{cc}
\hline Parameter & Value \\
\hline$\beta$ & $0.50 \pm 0.02$ \\
$\gamma$ & $1.11 \pm 0.05$ \\
$\nu$ & $0.70 \pm 0.05$ \\
$T_{C}$ & $(468 \pm 4) \mathrm{K}$ \\
\hline
\end{tabular}

Table 6.6. Critical exponents and Curie temperature of $\mathrm{Nd}_{60} \mathrm{Fe}_{30} \mathrm{Al}_{10}$ bulk samples according to Arrott analysis.

structural disorder, they indicate that the Curie temperature can be well defined at about $468 \mathrm{~K}$.

\subsubsection{Mössbauer Spectroscopy}

Beyond the different scattering techniques employed for the characterization of the bulk samples, a spectroscopic methods has been used to investigate the two-phases system of the as-cast bulk specimens: the ${ }^{57} \mathrm{Fe}$ Mössbauer spectroscopy. The Mössbauer effect permits to determine whether Fe atoms have a ferromagnetic surrounding or, instead, a paramagnetic one through a spectroscopic analysis.
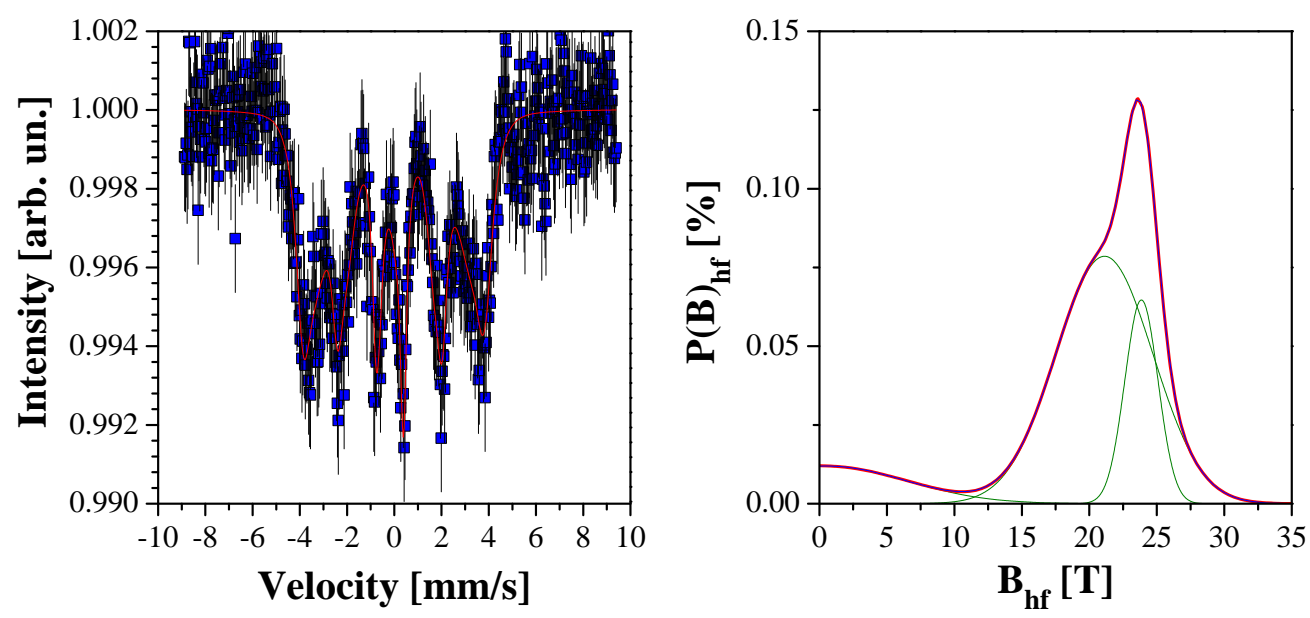

Figure 6.24. (Left) ${ }^{57} \mathrm{Fe}$ Mössbauer spectrum of $\mathrm{Nd}_{60} \mathrm{Fe}_{30} \mathrm{Al}_{10}$ bulk sample taken at room temperature ( $\square)$. The line $(-)$ results from the fitting procedure. (Right) The resultant hyperfine field distribution (-) is the sum $(-)$ of a (super-)paramagnetic and two ferromagnetic contributions $(-)$.

Figure 6.24 shows a Mössbauer spectrum recorded at room temperature (no magnetic field has been employed). The considerable broadening of the lines is typical for amorphous alloys. The experimental spectrum can be fitted with a superposition of a (super-) paramagnetic and two ferromagnetic contributions. The fit shows that about $18 \%$ of the Fe atoms may be attributed to ferromagnetic sites with hyperfine field $B_{h f}=23.9$ T, $65 \%$ 
to ferromagnetic sites with $B_{h f}=21.1 \mathrm{~T}$, and only $16 \%$ to (super-)paramagnetic ones $\left(B_{h f}=0.5 \mathrm{~T}\right)$. This result indicates that about $84 \%$ of the Fe sites has a ferromagnetic surrounding and suggests, in good agreement with the structural characterization, that the phase which is magnetic at room temperature is Fe-rich while the phase ordering at lower temperature must have a reduced Fe concentration and, hence, Nd-rich.

\subsubsection{Differential Scanning Calorimetry and Thermal Stability}

The results presented in section 6.1.1 indicate that the $\mathrm{Nd}_{60} \mathrm{Fe}_{30} \mathrm{Al}_{10}$ bulk samples are intrinsic composites and consequently metastable systems. As seen in chapter 3 , several possible transformations may drive a system towards a stable state and the technique commonly employed to study the transformation paths in a metastable material is the differential scanning calorimetry (see section 5.5).

Figure 6.25 shows a DSC scan of a $\mathrm{Nd}_{60} \mathrm{Fe}_{30} \mathrm{Al}_{10}$ bulk sample measured with a heating rate $\beta$ of $5 \mathrm{~K} / \mathrm{min}$ between 300 and $850 \mathrm{~K}$. The curve presents a single step crystallization process with a maximum transformation rate at temperature $T_{p}=761 \mathrm{~K}$ and an enthalpy release $\Delta H=20.96 \mathrm{~J} / \mathrm{g}$, while no glass transition can be clearly detected. This fact is

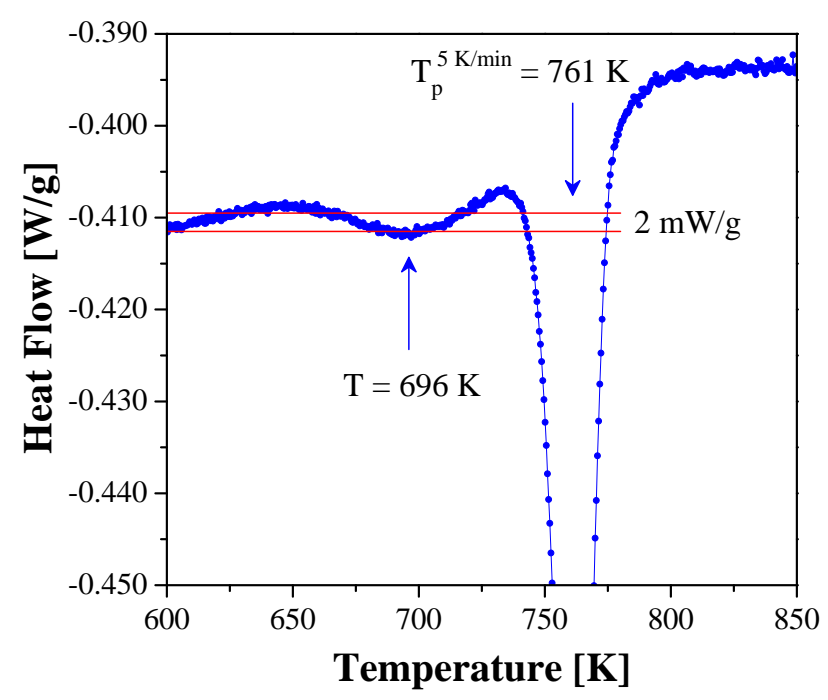

Figure 6.25. DSC scan of a $\mathrm{Nd}_{60} \mathrm{Fe}_{30} \mathrm{Al}_{10}$ bulk sample measured with an heating rate of $5 \mathrm{~K} / \mathrm{min}$. The arrows indicate the peak temperatures of two exothermic processes. $T_{p}=761$ is the crystallization temperature of the amorphous phase.

due to a parallel exothermic process which is activated at temperatures close to the calorimetric glass temperature and whose signal covers the endothermic glass transition. $\mathrm{Li} 107$ reported evidences of two distinct processes in the thermodynamic behavior of $\mathrm{Nd}_{60} \mathrm{Fe}_{30} \mathrm{Al}_{10}$ samples in the temperature range from 550 to $650 \mathrm{~K}$ : a pre-crystallization $\left(T^{\text {onset }}=596 \mathrm{~K}, T_{p}=614 \mathrm{~K}, \beta=5 \mathrm{~K} / \mathrm{min}\right)$ and the glass transition $\left(T_{g}=591 \mathrm{~K}\right)$, which 
could be detected only by modulated differential calorimetry ${ }^{(6)}$.

The complexity of the DSC curve implies that the thermal stability of the $\mathrm{Nd}_{60} \mathrm{Fe}_{30} \mathrm{Al}_{10}$ alloy must be addressed in more details. For this scope, the crystallization process has been characterized by applying the Kissinger method to the kinetic of the reaction (see paragraph 3.1.1 in section 3.1, while the processes close to the glass transition have been investigated by in situ high-temperature HE-WAXS (see section 5.6).
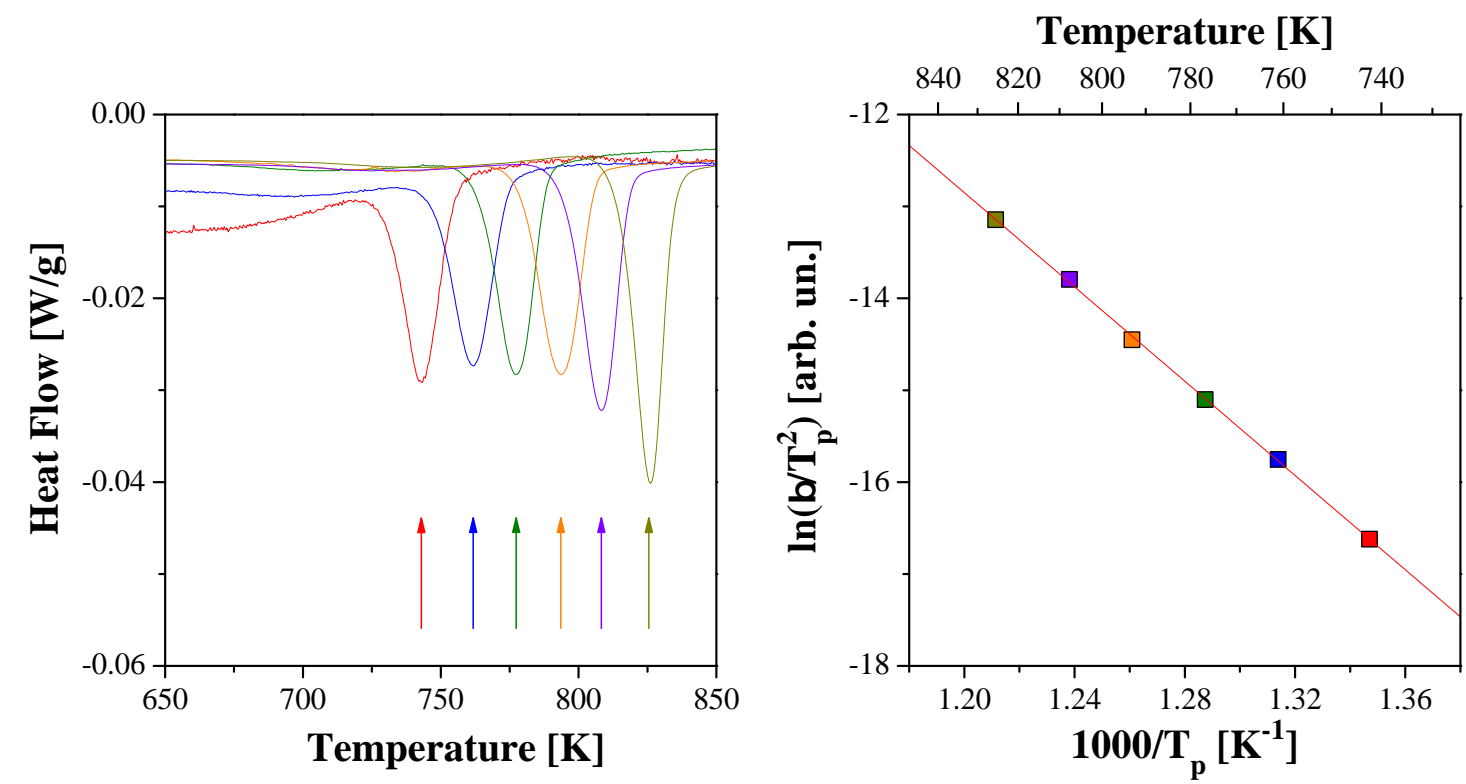

Figure 6.26. (Left) DSC scans of $\mathrm{Nd}_{60} \mathrm{Fe}_{30} \mathrm{Al}_{10}$ bulk samples measured at $2(-), 5(-), 10$ $(-), 20(-), 40(-)$, and $80 \mathrm{~K} / \mathrm{min}(-)$. Each curve has been normalized by the relative heating rate. (Right) Kissinger plot obtained from the analysis of the DSC scans measured at different heating rates. The linear fit $(-)$ is in accordance with equation 3.27 .

According to equation 3.27, the activation energy $\left(\Delta E_{c}\right)$ and the frequency factor $\left(K_{0}\right)$ which describes the kinetic of a crystallization process can be calculated taking into account the shift of the transformation peaks as a function of the heating rate in DSC measurements. Figure 6.26 (left) shows several differential calorimetric scans which have been recorded at $2,5,10,20,40$, and $80 \mathrm{~K} / \mathrm{min}$. The Kissinger plot $\ln \left(\beta / T_{p}^{2}\right)$ vs. $1 / T_{p}$ which has been deduced from the DSC data is presented on the right of figure 6.26. A fit of this curve provides the two unknown parameters $\Delta E_{c}$ and $K_{0}$. Their values are listed in table 6.7 [15]. Compared to other metallic glasses, the activation energy for the $\mathrm{Nd}_{60} \mathrm{Fe}_{30} \mathrm{Al}_{10}$ alloy is relatively low $\left(\mathrm{Cu}_{60} \mathrm{Hf}_{25} \mathrm{Ti}_{15} \Delta E_{c}=3.94 \mathrm{eV}\right.$ [109], $\mathrm{Fe}_{62.5} \mathrm{Ni}_{7.5} \mathrm{Co}_{6} \mathrm{Zr}_{6} \mathrm{Nb}_{2} \mathrm{Cu}_{1} \mathrm{~B}_{15} \Delta E_{c}=3.4 \mathrm{eV}$ and $\mathrm{Fe}_{40} \mathrm{Ni}_{40} \mathrm{~B}_{20} \Delta E_{c}=4.2 \mathrm{eV}$ [14]) and it seems to indicate a reduced thermal stability and a higher tendency of the amorphous phase to crystallize.

\footnotetext{
${ }^{(6)}$ The values of $T^{\text {onset }}$ and $T_{p}$ published by $\mathrm{Li} 107$ are lower than those found in this work. Those differences may be due to the different casting conditions which were used to prepare the samples
} 


\begin{tabular}{cc}
\hline Parameter & Value \\
\hline$\Delta E_{c}$ & $(2.21 \pm 0.01) \mathrm{eV}$ \\
$K_{0}$ & $(1.65 \pm 0.15) \cdot 10^{12} \mathrm{~s}^{-1}$ \\
\hline
\end{tabular}

Table 6.7. Kissinger parameters found for $\mathrm{Nd}_{60} \mathrm{Fe}_{30} \mathrm{Al}_{10}$ bulk samples according to equation 3.27 .

Such behavior can be graphically expressed by a continuous heating transformation diagram (see section 3.1.2). Figure 6.27 shows the CHT curve deduced from the Kissinger analysis and from equation 3.27 . The set of points $\left\{t_{p}, T_{p}\right\}$ allows to predict the crystallization temperature of the $\mathrm{Nd}_{60} \mathrm{Fe}_{30} \mathrm{Al}_{10}$ alloy for any heating rate and without running DSC measurement. For a heating rate $\beta=4 \mathrm{~K} / \mathrm{min}$ the crystallization will take place, for example, at $T_{p}=757 \mathrm{~K}$.

Knowing this temperature is of fundamental importance for the high-temperature insitu $\mathrm{x}$-ray diffraction, since HE-WAXS measurements have been recorded between 301 and $814 \mathrm{~K}$ upon heating with at $4 \mathrm{~K} / \mathrm{min}$. Several complete Debye-Scherrer spectra, as that shown in figure 6.2, have been therefore recorded in temperature scan mode as explained in section 5.6. The isotropic nature of the 2-D WAXS patterns allows also in this case an angular averaging of the recorded intensity. Figure 6.28 reports the resultant onedimensional curves showing the scattering intensity as function of the scattering vector $Q$ and of the sample temperature.

In analogy to the analysis of the room-temperature curve, the mean size of the $\mathrm{Nd}$ nanocrystals has been calculated by fitting the (110) reflection and applying equation 5.86 to the resultant value of the FWHM. Figure 6.29 (left) shows the temperature evolution of the mean grain radius $r(T)$. From room temperature up to about $600 \mathrm{~K}$ no appreciable difference of the grain size is detectable, however, as the sample temperature further increases, $r(T)$ slowly increases suggesting the activation of a grain growth process. The growth continues clearly up to $752 \mathrm{~K}$ when, in good accordance with the Kissinger analysis $\left(T_{p}=757 \mathrm{~K}\right)$, the amorphous phase crystallizes limiting therefore the further growth of the Nd nanocrystals. The crystallization is confirmed also from the appearance of additional reflections which cannot be indexed with a dhcp structure.

In order to distinguish whether the observed grain growth is mainly a nucleationand-growth (section 3.1) or a coarsening process (section 3.2), the temperature evolution of the volume fraction of the crystalline phase must be estimated. In this context, the volume fraction has been expressed qualitatively as the ratio $\alpha$ between the integral area of the (110) diffraction peak and the integral background intensity (see equation 5.90). As shown in figure 6.29 (right), the ratio $\alpha$ remains nearly constant up to about $740 \mathrm{~K}$ suggesting that coarsening is the leading process responsible for the grain size evolution $r(T)$. As previously seen in section 3.2 .1 , the minimization of the interfacial free energy 


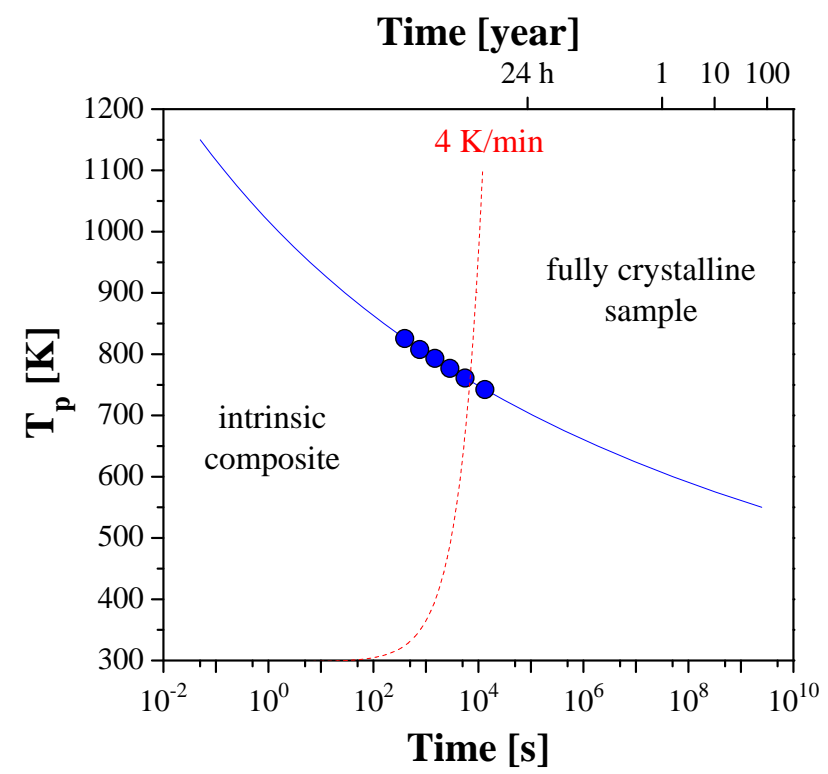

Figure 6.27. Transformation diagram for the $\mathrm{Nd}_{60} \mathrm{Fe}_{30} \mathrm{Al}_{10}$ sample as derived from equation 3.27 under the condition $t_{p}=\left(T_{p}-T_{0}\right) / \beta(-)$. The circles $(\bullet)$ indicate the experimen$\operatorname{tal} T_{p}$ 's while the dashed line (--) shows the temperature evolution during a non-isothermal annealing process at $4 \mathrm{~K} / \mathrm{min}$.

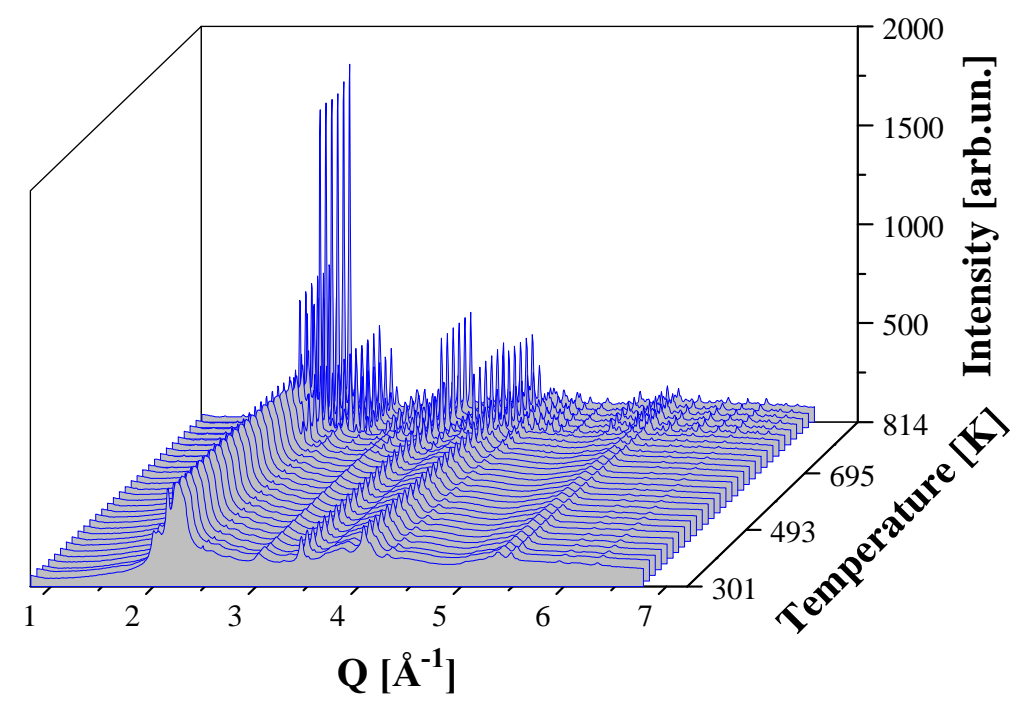

Figure 6.28. HE-WAXS diffraction patterns of a $\mathrm{Nd}_{60} \mathrm{Fe}_{30} \mathrm{Al}_{10}$ bulk sample during in-situ annealing. The diffraction intensity is plotted as function of the scattering vector $Q$ and of the sample temperature. 

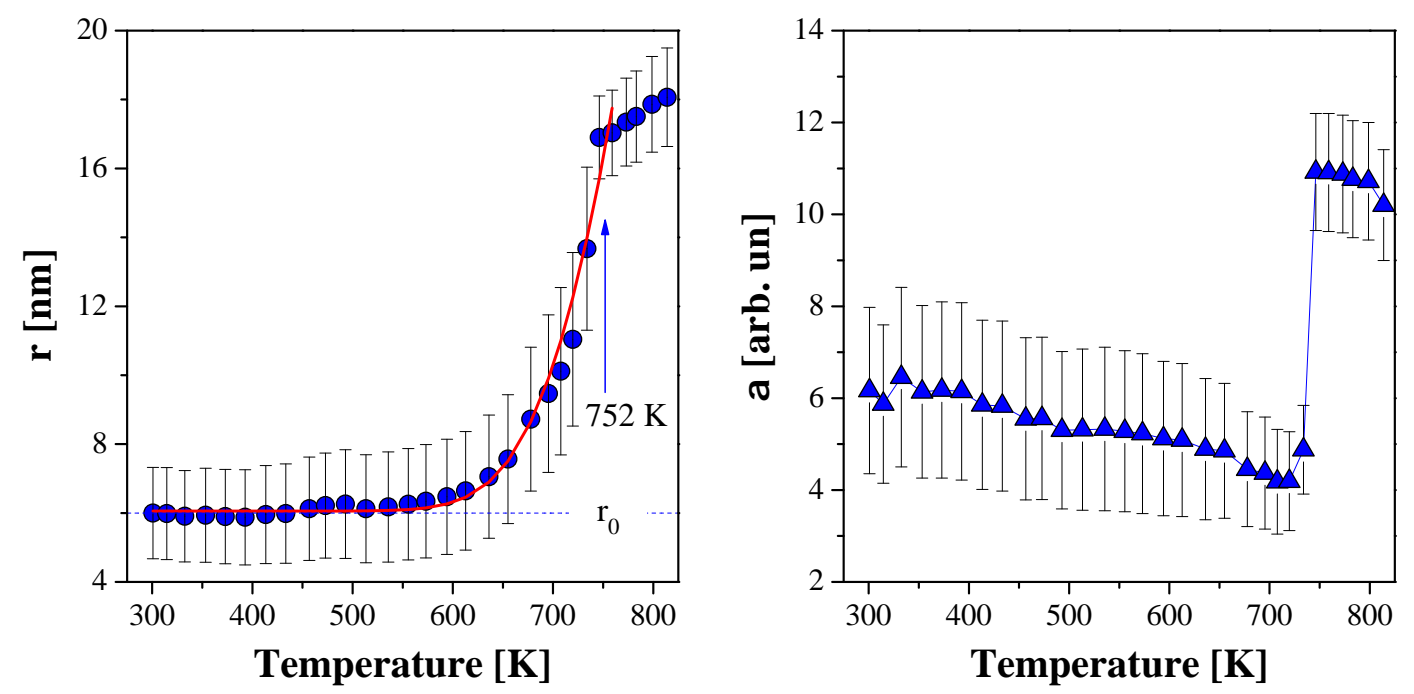

Figure 6.29. (Left) Temperature evolution of the mean grain radius $r(\boldsymbol{\square})$ in a continuous heating mode experiment (heating rate $4 \mathrm{~K} / \mathrm{min}$ ). The solid line represents the fitting curve (-) according to equation 3.46 . (Right) The ratio $\alpha$ between the integral area of the (110) Bragg peak and the integral background intensity as function of the sample temperature

$(\Delta)$.

can be considered in good approximation to be the main driving force for coarsening and it explains why larger grains grow at the expense of smaller ones. In this case, the temperature evolution of the mean grain radius $r$ can be described by equation 3.46 (page 37)

$$
r(t)=\left(r^{n}(0)+\int_{0}^{T} \frac{k_{0}}{\beta T} \cdot \exp \left(-\frac{\Delta E_{c}}{k_{B} T}\right) d T\right)^{1 / n}
$$

where $r_{0}$ is the initial grain radius, $n$ the growth exponent, $k_{0}$ the frequency constant, and $\Delta E_{g}$ is the activation energy.

The solid line in figure 6.29 represents the fit to the experimental data up to $760 \mathrm{~K}$ according to equation 3.46. A good agreement between experimental curve and theoretical model is found for the values of $r_{0}, k_{0}, \Delta E_{g}$, and $n$ listed in table 6.8 and for $T_{0}=301 \mathrm{~K}$, which was considered constant. For the value of the growth exponent theory predicts

\begin{tabular}{cc}
\hline Parameter & Value \\
\hline$r_{0}$ & $6.05 \mathrm{~nm}$ \\
$k_{0}$ & $9 \cdot 10^{9} \mathrm{~nm}^{2} \mathrm{~K} / \mathrm{s}$ \\
$\Delta E_{g}$ & $1.08 \mathrm{eV}$ \\
$n$ & 2.2 \\
\hline
\end{tabular}

Table 6.8. Fit parameters according to equation 3.46 and the grain coarsening process in $\mathrm{Nd}_{60} \mathrm{Fe}_{30} \mathrm{Al}_{10}$ bulk samples. 
$n=2$ for nucleation-and-growth process and $n=3$ for coarsening but, experimentally, values of $n$ between 1.5 and 4 have been observed $[34$.

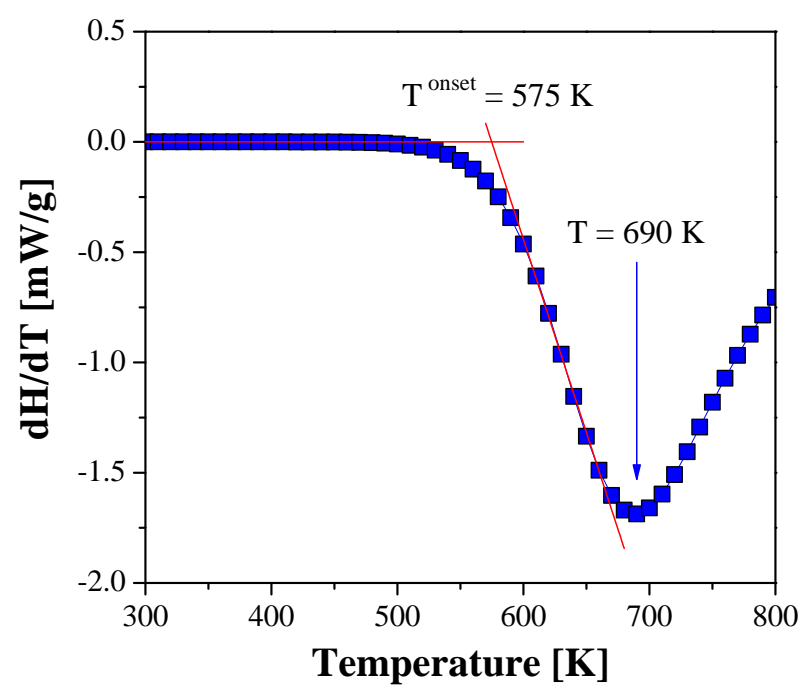

Figure 6.30. Calculated calorimetric signal $d H / d T$ arising from the interfacial enthalpy release during the coarsening process, according to equation 3.49. The arrow indicates the peak temperature of the process.

From the parameters of equation 3.46 it is possible to estimate the interfacial enthalpy which is released during the coarsening and, furthermore, to compare its variation rate with the DSC signal measured in the same temperature range. The magnitude of the calorimetric signal arising from the grain coarsening is given by equation 3.49 (page 38)

$$
\frac{d H}{d T}=-\frac{H_{0} r_{0}}{r(T)} \frac{d r}{d T}=-\left(\frac{H_{0} r_{0}}{n}\right) \frac{k(T)}{r^{n+1}}
$$

The temperature dependence of $d H / d T$ for the $\mathrm{Nd}_{60} \mathrm{Fe}_{30} \mathrm{Al}_{10}$ bulk sample is shown in figure 6.30 as obtained by substituting in the previous expression the values of $r_{0}, n, T_{0}, k_{0}$, and $\Delta E_{g}$ shown in table 6.8 , and assuming an interfacial energy $H_{0}=5 \mathrm{~J} / \mathrm{g}$ (normalized to $1 \mathrm{~g}$ of sample mass). The growth of the Nd nanocrystals contributes an exothermic signal $(d H / d t<0)$ which is released at $T^{\text {onset }}=575 \mathrm{~K}$ and reaches the maximum height of about $2 \mathrm{~mW} / \mathrm{g}$ at $T_{x}=690 \mathrm{~K}$. This result is in good agreement with the calorimetric data in figure 6.25 which shows a broad exothermic peak, prior to the crystallization of the amorphous phase, releasing a maximal heat of only some $\mathrm{mW} / \mathrm{g}$ at the peak temperature of $696 \mathrm{~K} 15$.

\subsubsection{Summary}

The results of the structural investigation on $\mathrm{Nd}_{60} \mathrm{Fe}_{30} \mathrm{Al}_{10}$ bulk samples have shown that those specimens are intrinsic composite materials consisting of a crystalline phase embedded in an amorphous matrix. The structure of the crystalline phase has been successfully 
identified by $\mathrm{x}$-ray diffraction as a double hexagonal closed-packed (dhcp) with lattice constants $a=(3.663 \pm 0.018) \AA$ and $c=(11.783 \pm 0.101) \AA$, in good accordance with those found for metallic Neodymium, suggesting that the nanocrystalline precipitates are Nd-rich. This conclusion has been confirmed by HRTEM investigation and EDX analysis which indicates that the nanocrystals have a Nd concentration larger than $80 \%$ while the chemical composition of the remanent amorphous matrix is close to the nominal one. Diffraction and electron microscopy have not indicated the presence of other crystalline phases.

The formation of the two phases is likely to arise from a phase separation process taking place in the melt or in the undercooled liquid. Phase separation is a direct consequence of chemical and thermodynamical properties of an alloy (differences of the heats of mixing, local minima in the composition dependence of the Gibbs free energy, atomic species diffusivity, etc.), and the cooling rate determines the path through phase separation and crystallization which further minimizes the Gibbs free energy. The crystallization of one phase and the consequent formation of $\mathrm{Nd}$-rich nanocrystals is the result of the minimization of the Gibbs free energy of the system which results to be, therefore, an intrinsic composite.

The morphology of the Nd-rich precipitates has been further investigated using smallangle neutron scattering which indicated that the crystalline phase forms an extended network over the whole sample and shows mass fractal features. The complex morphology has been well characterized by the mass distribution $M(r) \propto\left(r / r_{0}\right)^{d_{f}}$ (equation 6.3), with a mass fractal dimension $d_{f}$ equal to 2.5 in good agreement with the diffusion limited aggregation (DLA) model 19,139 .

The magnetic characterization has confirmed the presence of the two phases since they show different magnetic properties and ordering temperatures. The results have permitted to conclude that the amorphous matrix orders ferromagnetically at about $468 \mathrm{~K}$ while the crystalline phase shows a complex magnetic behavior with magnetic transitions at 10 and $33 \mathrm{~K}$. In the temperature range where the crystalline precipitates are paramagnetic, the bulk samples have shown hard magnetic behavior with a coercive field at room temperature of about $250 \mathrm{kA} / \mathrm{m}$. As the sample temperature decreases, the coercivity increases and its variation can be well understood taking into the Gaunt model for strong domainwall pinning effects 63 . At about $33 \mathrm{~K}$ (first ordering temperature of the low $-T_{C}$ phase) the coercive field decreases indicating a strong interplay between the pinning mechanisms and the magnetic properties of the nanocrystals.

The Gaunt analysis indicates that in order to show strong pinning, the pinning centers in the $\mathrm{Nd}_{60} \mathrm{Fe}_{30} \mathrm{Al}_{10}$ bulk samples should have a radius of about $7 \mathrm{~nm}$. This value has been effectively verified by the use of polarized small-angle neutron scattering (pSANS) and the separation of magnetic and nuclear contrasts. The pSANS results have moreover indicated a strong variation of the magnetic properties at the interfaces between the 
amorphous matrix and the nanocrystals aggregates which can be described therefore in terms of a core-shell model. As schematically shown in figure 6.31, according to the

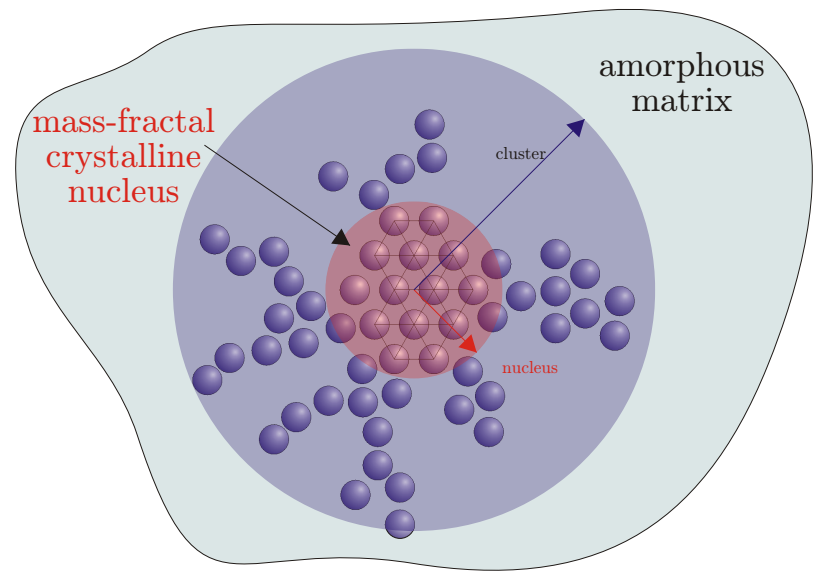

Figure 6.31. Core-shell model proposed for the interpretation of the magnetic behavior and of the strong pinning processes in $\mathrm{Nd}_{60} \mathrm{Fe}_{30} \mathrm{Al}_{10}$ bulk samples.

structural and magnetic results the nanocrystalline clusters may be thought as composed of a paramagnetic nucleus of size $\approx 14 \mathrm{~nm}$ (radius $\xi_{\text {magn }}=7 \mathrm{~nm}$ ) and a ferromagnetic shell whose thickness is approximately $12 \mathrm{~nm}\left(\xi_{\text {nucl }}=19 \mathrm{~nm}\right)$ and whose chemical composition is close to that of the central nucleus. The entire cluster is a mass fractal with fractal dimension 2.5 as the fit of pSANS curve has also confirmed. The magnetic properties of the shell differs from those of the matrix and such differences are responsible for local variation of the domain-wall energy density $\gamma_{w}$ and, hence, for the pinning forces which determine the observed pinning behavior. The size of the nucleus is in good accordance with the value found by $\mathrm{x}$-ray diffraction for the size of the $\mathrm{Nd}$-rich nanocrystals $(13 \mathrm{~nm})$.

The $\mathrm{Nd}_{60} \mathrm{Fe}_{30} \mathrm{Al}_{10}$ intrinsic composite has been further investigated to determine the evolution of the structural properties upon annealing. In the as-cast state the crystalline grains have a mean lateral dimension of about $13 \mathrm{~nm}$ and undergo a coarsening process at temperatures well below the crystallization temperature of the amorphous phase. The combination of in-situ high temperature $\mathrm{x}$-ray diffraction and calorimetric methods have permitted to calculate their activation energies. In the case of the grain coarsening it results $\Delta E_{g}=1.08 \mathrm{eV}$ which is about half the energy involved in the transformation of the glassy matrix $\left(\Delta E_{c}=2.21 \mathrm{eV}\right)$ and a peak temperature of $690 \mathrm{~K}$ which is well below the crystallization temperature $T_{p}=757 \mathrm{~K}$ which has been determined by the timetransformation diagram and the Kissinger analysis. Furthermore it has been possible to evaluate the temperature and quantitatively estimate the enthalpy release associated with the grain size evolution, confirming the overlap with the endothermic glass transition which cannot therefore be observed by traditional scanning calorimetry in agreement with Li [107]. These results show the relevance of the combination of high-energy $x$-ray diffraction and differential scanning calorimetry in the study of intrinsic composites and 
suggests a possible wider use of this method to a larger class of materials undergoing complex structural evolution 15 .

Furthermore, the coarsening process may offer the possibility to vary morphology and mean grain size of the crystalline phase and hence to control the structural and magnetic properties which determine the strength of the pinning effects. Annealing at temperatures below the fully crystallization of the amorphous phase may tailor the hard magnetic behavior of the $\mathrm{Nd}_{60} \mathrm{Fe}_{30} \mathrm{Al}_{10}$ bulk samples.

\subsection{Master Alloy}

Samples called "master-alloy" are small spheres of about $2 \mathrm{~mm}$ in diameter (see figure 6.32 and of nominal composition $\mathrm{Nd}_{60} \mathrm{Fe}_{30} \mathrm{Al}_{10}$ which have been prepared by melt-quenching in an arc melter. The effective cooling rate of this preparation method is only some $\mathrm{K} / \mathrm{s}$

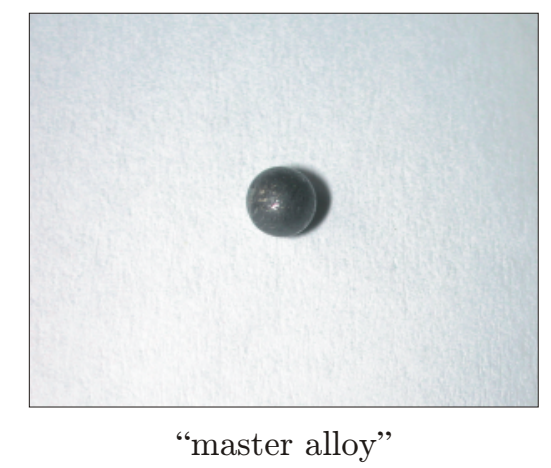

Figure 6.32. Picture of a "master alloy" sphere with a diameter about of $2 \mathrm{~mm}$.

and, hence, lower than that of the bulk samples $\left(10^{2} \mathrm{~K} / \mathrm{s}\right)$.

The differences of the structural and magnetic properties between these two types of specimens, which may derive from the different cooling rates, have been investigated by high-energy x-ray diffraction, differential scanning calorimetry, and DC magnetometry.

\subsubsection{X-Ray Diffraction}

The x-ray diffraction spectra of a "master-alloy" have been measured by high-energy $\mathrm{x}$-ray scattering on $\mathrm{a} \approx 0.5 \mathrm{~mm}$ thick specimen which has been cut from an as-prepared sample. In analogy to the results on the bulk $\mathrm{Nd}_{60} \mathrm{Fe}_{30} \mathrm{Al}_{10}$, the WAXS 2-D pattern results to be perfectly isotropic and shows the presence of concentric Debye rings which overlap liquid-like halos. The angular averaging of the scattering intensity on the $2-\mathrm{D}$ detector permits to deduce the one dimensional spectrum which is shown in figure 6.33 . The curve indicates that the master-alloy is an intrinsic composite material built up of an amorphous matrix embedding random-oriented nanocrystals. The crystalline reflections 


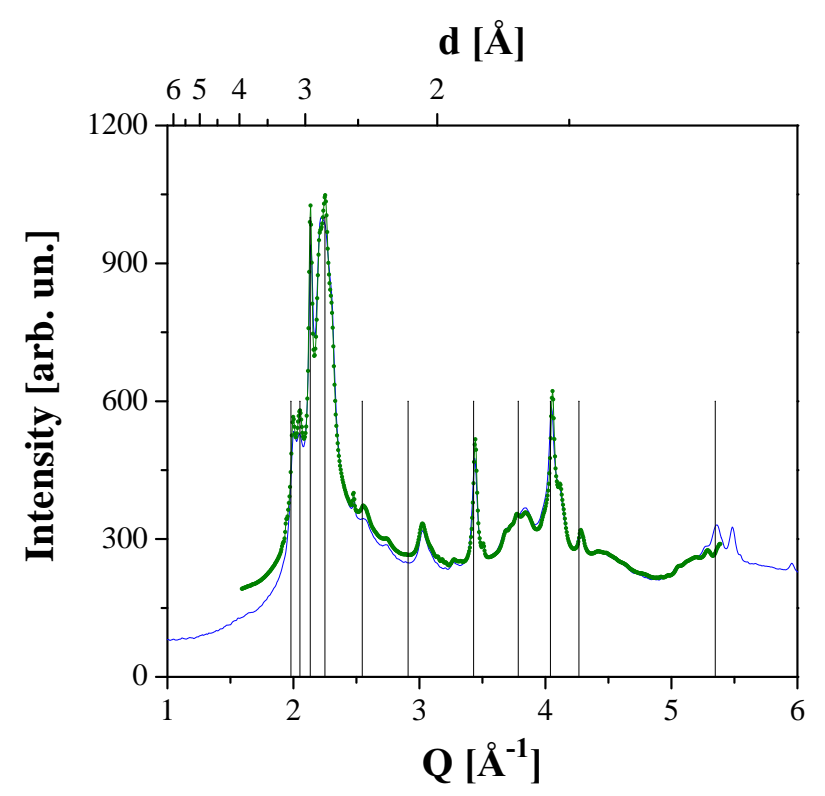

Figure 6.33. Averaged diffraction intensity vs. scattering vector $Q$ of a $\mathrm{Nd}_{60} \mathrm{Fe}_{30} \mathrm{Al}_{10}$ master-alloy measured by WAXS at room temperature $(-)$. The vertical lines $(-)$ indicate the diffraction $Q$-values of a dhcp structure with lattice constants $a=(3.676 \pm 0.021) \AA$ and $c=(11.691 \pm 0.107) \AA$. The curve recorded for the bulk sample $(-)$ is shown for comparison.

are indexed with a dhcp structure with lattice parameters $a=(3.676 \pm 0.021) \AA$ and $c=(11.691 \pm 0.107) \AA$. The values of the lattice parameters indicate clearly that the precipitates are $\mathrm{Nd}$-rich nanocrystals and suggest that structure and chemical composition of the crystalline phase does not differ from the phase observed in the bulk sample.

Furthermore, the estimation of the mean grain size and of the volume fraction of the nanocrystals gives the values $t=(15 \pm 1) \mathrm{nm}$ and roughly $x=16 \%$ (due to a crystallinity of about $39 \%$ ) and, in comparison with the results of the bulk sample (see section 6.1.1), indicates that only minor differences in the structural properties of the two specimens exist.

\subsubsection{Differential Scanning Calorimetry and Thermal Stability}

Although the structural analysis has shown a low dependence of the sample properties from the cooling rate, it is worth to notice that $\mathrm{x}$-ray diffraction is less sensible to small differences and to local variations of the physical features of an intrinsic composite than other techniques. One of them is the differential scanning calorimetry.

In section 6.1.7, the thermal stability of a bulk $\mathrm{Nd}_{60} \mathrm{Fe}_{30} \mathrm{Al}_{10}$ composite has been determined by DSC measurements at different heating rates and by applying the Kissinger method to the data. The same analysis has been used for the master alloy, and several DSC curves have been therefore recorded employing heating rates between 10 and $160 \mathrm{~K} / \mathrm{min}$. 
The Kissinger plot is shown in figure 6.34 together with the experimental data of the bulk

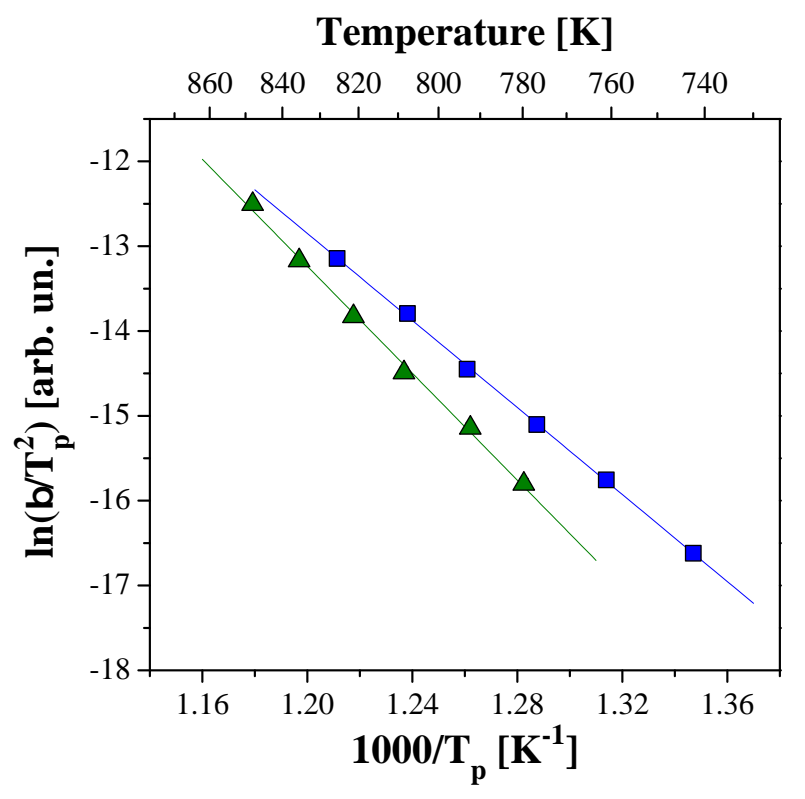

Figure 6.34. Kissinger plots of $\mathrm{Nd}_{60} \mathrm{Fe}_{30} \mathrm{Al}_{10}$ master alloy $(\boldsymbol{\Delta})$ and bulk ( $\left.\boldsymbol{\square}\right)$ samples, respectively. The lines represents the relative fits according to equation 3.27 .

sample. The linear regression allows the estimation of the two unknown parameters $\Delta E_{c}$ and $K_{0}$ (see equation 3.27 ) whose values are listed in table 6.9. The activation energy

\begin{tabular}{ccc}
\hline \multirow{2}{*}{ Parameter } & Master Alloy & Bulk \\
\hline$\Delta E_{c}$ & $(2.72 \pm 0.01) \mathrm{eV}$ & $(2.21 \pm 0.01) \mathrm{eV}$ \\
$K_{0}$ & $(1.51 \pm 0.06) \cdot 10^{15} \mathrm{~s}^{-1}$ & $(1.65 \pm 0.15) \cdot 10^{12} \mathrm{~s}^{-1}$ \\
\hline
\end{tabular}

Table 6.9. Parameters deduced from the Kissinger analysis of $\mathrm{Nd}_{60} \mathrm{Fe}_{30} \mathrm{Al}_{10}$ "master alloy" and bulk samples according to equation 3.27 .

and the frequency factor of the master alloy result to be larger than for the bulk sample indicating different thermodynamic behaviors. In fact, as it merges from section 3.1.1, $\Delta E_{c}$ and $K_{0}$ are directly related to the kinematic and thermodynamic (e.g., diffusivity, crystallization, etc.) properties of a specimen and the differences which arise from the Kissinger analysis indicate therefore a different thermal stability of the amorphous phase upon thermal treatments and, hence, a different tendency to crystallize. Such differences are clearly evidenced in figure 6.35 where the continuous heating transformation diagrams of the two samples deduced from the previous Kissinger analysis are shown.

The results suggest that the amorphous phase of the master alloy differs from that of the bulk sample and prove that the physical properties of the $\mathrm{Nd}_{60} \mathrm{Fe}_{30} \mathrm{Al}_{10}$ intrinsic composites are cooling rate dependent (e.g., $T_{p}^{\text {bulk }}=761 \mathrm{~K}, T_{p}^{\text {master alloy }}=780 \mathrm{~K}$ for heating rate of $5 \mathrm{~K} / \mathrm{min}$ ). 


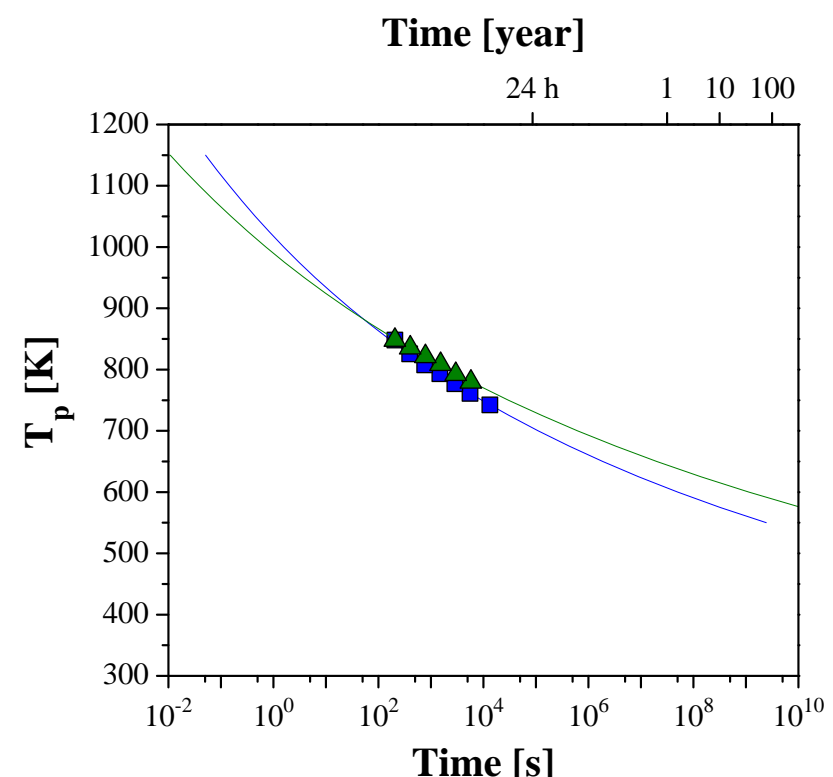

Figure 6.35. Transformation diagram for a $\mathrm{Nd}_{60} \mathrm{Fe}_{30} \mathrm{Al}_{10}$ master alloy (-) and bulk sample $(-)$ as derived from equation 3.27 . The symbols indicate the relative crystallization temperatures $T_{p}^{\beta}$ which have been measured at different heating rates $\beta$.

\subsubsection{Magnetometry}

In order to determine the effects of the different cooling rates on the magnetic properties, the $\mathrm{Nd}_{60} \mathrm{Fe}_{30} \mathrm{Al}_{10}$ master alloy has been characterized by SQUID and by vibrating-sample magnetometry between 4 and $600 \mathrm{~K}$.

Figure 6.37 shows the hysteresis loop of a master alloy specimen measured at room temperature with a maximal applied field $\mu_{0} H=5 \mathrm{~T}$ and, for comparison, the curve of the bulk sample recorded in the same conditions. The magnetic behavior of the two specimens differs significantly although a comparable maximal magnetization at $5 \mathrm{~T}$ has been measured. The values of $M^{5 \mathrm{~T}}$, of the remanence $M_{r}$, of the coercivity $H_{c}$, and of the product $(\mu 0 M \cdot H)_{\max }$ for the master alloy are listed in table 6.10. The measured

\begin{tabular}{ccc}
\hline \multirow{2}{*}{ Parameter } & \multicolumn{2}{c}{ Value } \\
& Master Alloy & Bulk \\
\hline$M^{5 \mathrm{~T}}$ & $184 \mathrm{kA} / \mathrm{m}$ & $183 \mathrm{kA} / \mathrm{m}$ \\
$M_{r}$ & $76 \mathrm{kA} / \mathrm{m}$ & $93 \mathrm{kA} / \mathrm{m}$ \\
$H_{c}$ & $142 \mathrm{kA} / \mathrm{m}$ & $246 \mathrm{kA} / \mathrm{m}$ \\
$\left(\mu_{0} M \cdot H\right)_{\max }$ & $4 \mathrm{~kJ} / \mathrm{m}^{3}$ & $19 \mathrm{~kJ} / \mathrm{m}^{3}$ \\
\hline
\end{tabular}

Table 6.10. Values of the magnetization at $5 \mathrm{~T} M^{5 \mathrm{~T}}$, of the remanence $M_{r}$, of the coercivity $H_{c}$, and of the product $\left(\mu_{0} M \cdot H\right)_{\max }$ measured at $300 \mathrm{~K}$ for a $\mathrm{Nd}_{60} \mathrm{Fe}_{30} \mathrm{Al}_{10}$ master alloy. The correspondent values for the bulk sample are shown for comparison. 
Field [kOe]

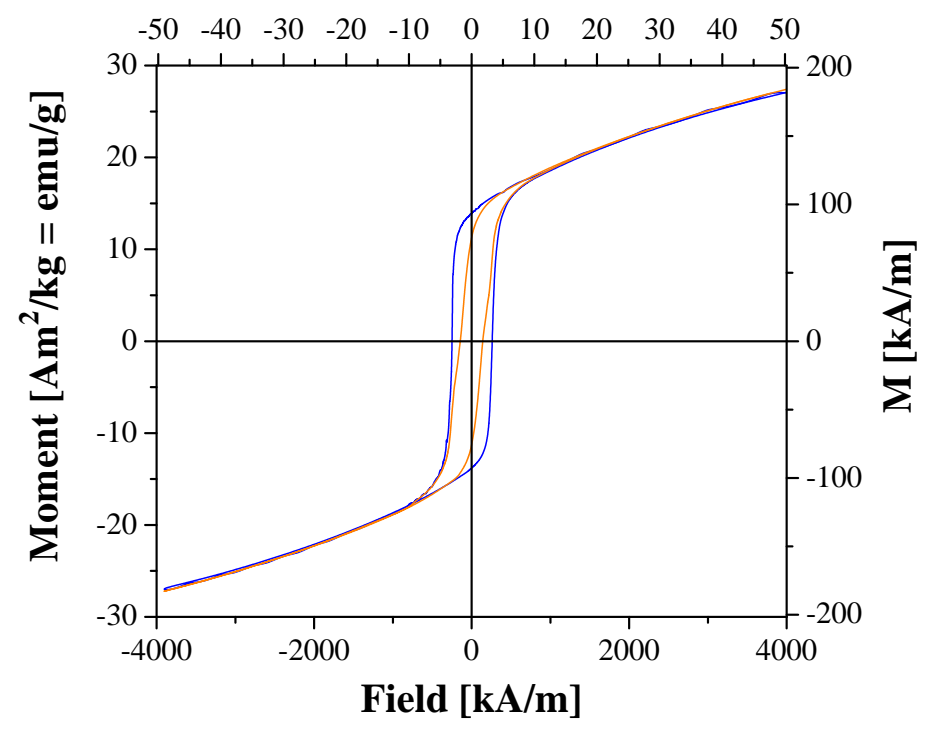

Figure 6.36. Hysteresis loops of a $\mathrm{Nd}_{60} \mathrm{Fe}_{30} \mathrm{Al}_{10}$ "master alloy" $(-)$ and of a bulk sample (一) recorded at $300 \mathrm{~K}$.

coercive field is only $142 \mathrm{kA} / \mathrm{m}$, in comparison with the $246 \mathrm{kA} / \mathrm{m}$ of the bulk sample and, as it can be deduced from figure 6.36, the product $\left(\mu_{0} M \cdot H\right)_{\max }$ becomes approximately $4 \mathrm{~kJ} / \mathrm{m}^{3}$.

Figure 6.37 (left) shows a FC thermomagnetic curve of an as-prepared sample measured between 4 and $600 \mathrm{~K}$ and with an external field of $1 \mathrm{~T}$. Two ferromagnetic-toparamagnetic transitions appear at about 50 and $500 \mathrm{~K}$ indicating, in analogy with the bulk sample, the ordering temperatures of the crystalline phase and of the amorphous matrix, respectively. The normalized temperature derivative $(d M / d T) / M$ shown in figure 6.37 (right) indicate clearly that the low $-T_{C}$ is however the consequence of two ordering processes as previously deduced in section 6.1 .3 . These results seem to indicate that the magnetic behavior of this sample is very similar to that of the bulk specimen and, except minor differences, the magnetization mechanisms which control their properties seem to be identical.

In order to verify this hypothesis and to understand how the structural and magnetic characteristics of the master alloy influence the coercivity, several hysteresis loops have been measured between 4 to $400 \mathrm{~K}$. From their analysis, the temperature evolution of the coercive field $H_{c}$ plotted in figure 6.38 (left) has been derived. Assuming that the strong increase of the coercivity as the temperature decreases is due to thermal activated processes and that pinning effects are responsible for the intrinsic value of $H_{c}$ at $T=0 \mathrm{~K}$, the Gaunt formalism has been applied to characterize the curve $H_{c}$ vs. T. The Gaunt plot $H_{c}^{1 / 2}$ vs. $T^{2 / 3}$ of the master alloy is shown in figure 6.38 (right). As the linearity of the curve above $20 \mathrm{~K}$ suggests, pinning effects define the coercive field of the sample in 

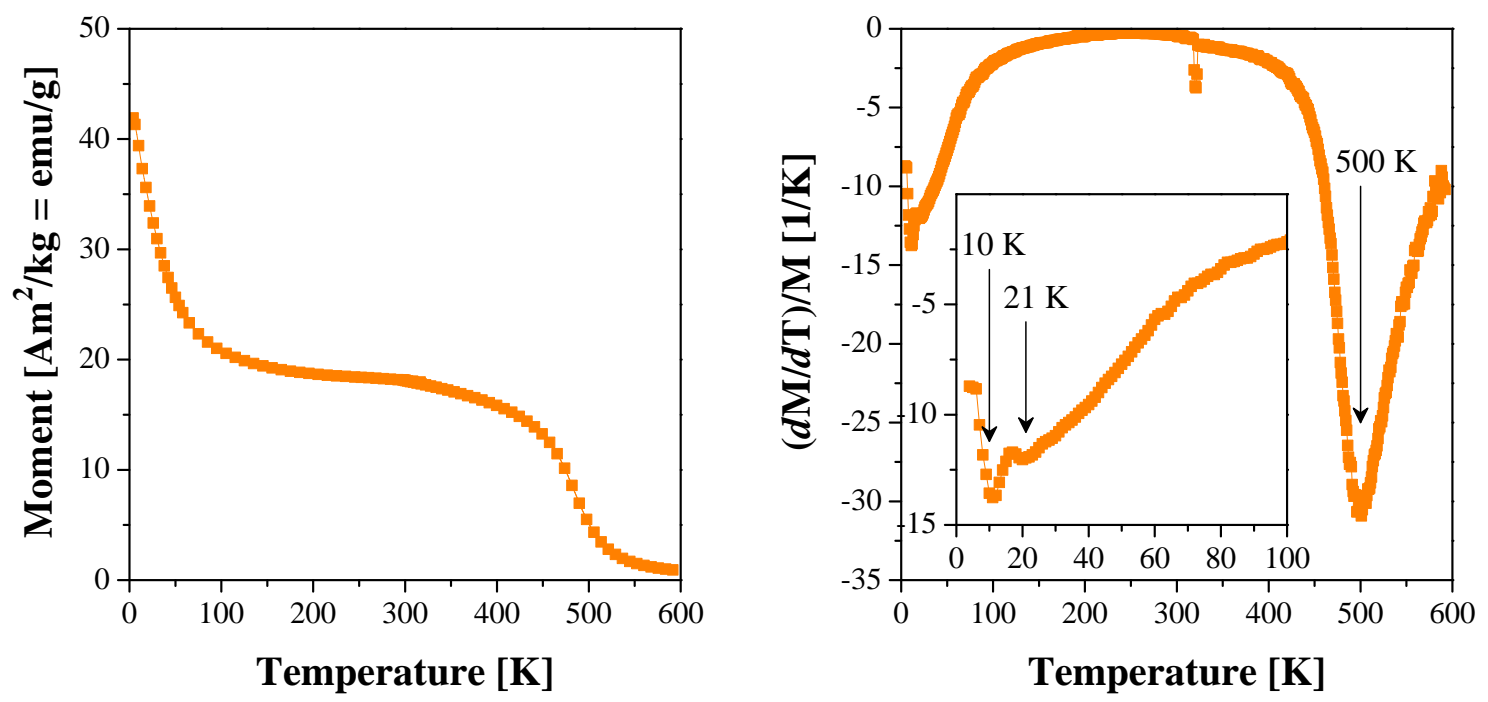

Figure 6.37. (Left) Temperature dependence of the magnetic moment of a field-cooled $(1 \mathrm{~T})$ "master alloy" sample measured with a SQUID and a VSM magnetometer in the range from 4 to $600 \mathrm{~K}$. (Right) The normalized derivative $(d M / d T) / M$ vs. $T$ curve. The arrows indicate the magnetic transition temperatures.
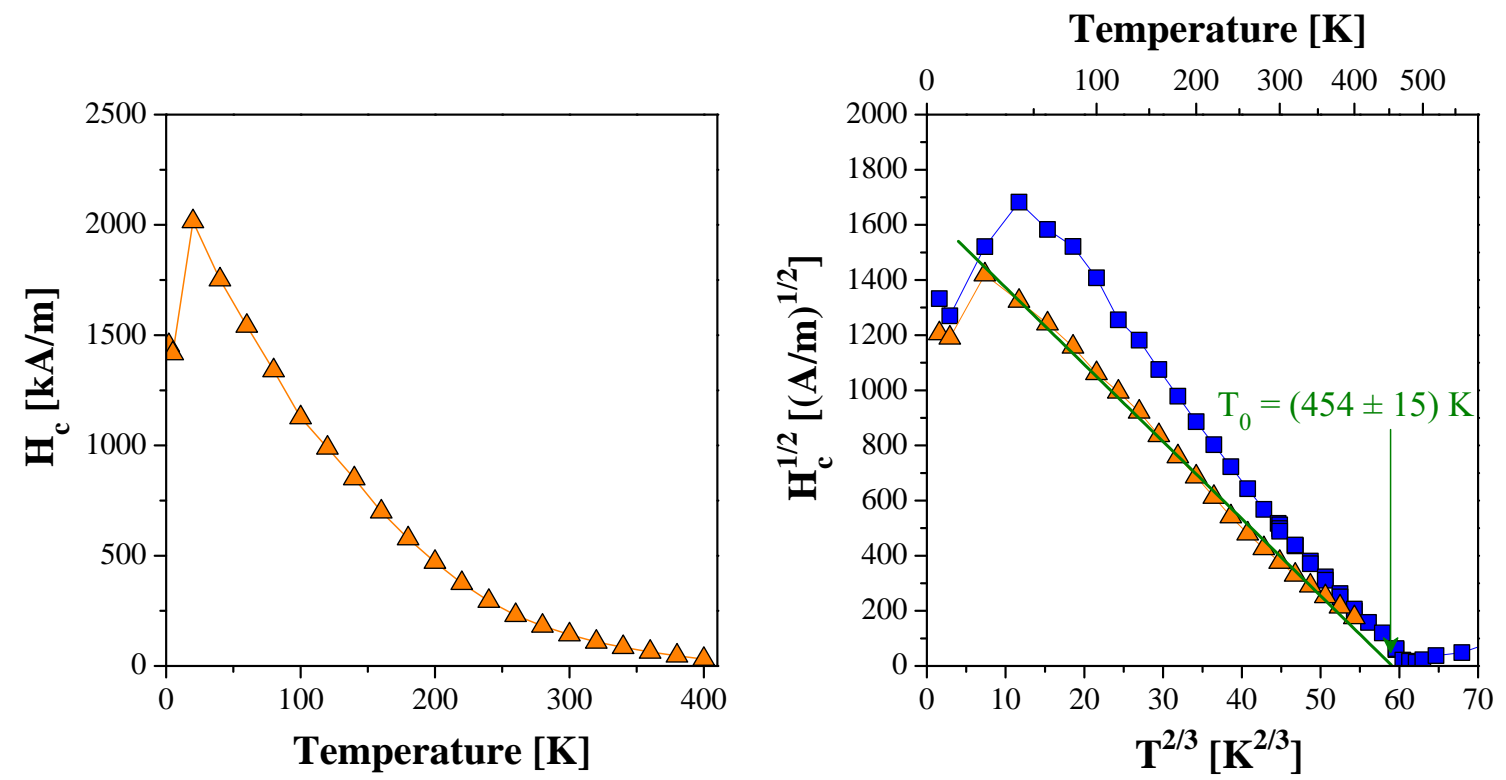

Figure 6.38. (Left) Temperature dependence of the coercivity $H_{c}$ of a master alloy sample. (Right) Gaunt plot $H_{c}^{1 / 2}$ vs. $T^{2 / 3}$ of the experimental data shown on the left $(\Delta)$ and of the bulk sample data ( $\boldsymbol{\square})$ (see figure 6.17). The line (-) represent the fit between 20 and $400 \mathrm{~K}$ according to the domain-wall pinning model [63]. The characteristic crossover temperatures $T_{0}$ is indicated by the arrow. 
agreement with the results of the bulk samples, however, the slope and $y$-axis intercept of the Gaunt plot are clearly lower than those of the bulk sample. The fit of the curve $H_{c}^{1 / 2}$ vs. $T^{2 / 3}$ permits to determine the values of $H_{0}$ and $T_{0}$ which are listed in table 6.11 . Compared to the parameters of the bulk samples, $H_{0}$ is clearly smaller suggesting that the

\begin{tabular}{ccc}
\hline \multirow{2}{*}{ Parameter } & \multicolumn{2}{c}{ Malue } \\
& Master Alloy & Bulk \\
\hline$H_{0}$ & $(2730 \pm 46) \mathrm{kA} / \mathrm{m}$ & $(4625 \pm 67) \mathrm{kA} / \mathrm{m}$ \\
$T_{0}$ & $(454 \pm 15) \mathrm{K}$ & $(451 \pm 12) \mathrm{K}$ \\
\hline
\end{tabular}

Table 6.11. Intrinsic coercive field $H_{0}$ and the crossover temperature $T_{0}$ as obtained by fitting the experimental data $H_{c}^{1 / 2}$ vs. $T^{2 / 3}$ of figure 6.38 according to equation 4.53 .

strength and the density of the pinning centers (and, hence, the intrinsic coercivity) are strongly reduced. On the contrary, $T_{0}$ remains unchanged suggesting that the thermalactivation process shows no dependence from the sample preparation method.

In analogy to the analysis proposed in section 6.1.4 pinning is strong if $A^{3 / 2} / K^{1 / 2} \ll$ $225 k_{B} T_{0} / 4 \pi^{4}$, however since $T_{0}^{\text {master alloy }}=T_{0}^{\text {bulk }}$, the ratio $A^{3 / 2} / K^{1 / 2}$ can be assumed to be equal to that of the bulk sample $\left(1 \cdot 10^{-22} \mathrm{~J}\right)$. Then, according to equation 6.11 , the density of pinning sites becomes

$$
\rho=8 \pi^{4} \frac{A^{3 / 2}}{K^{1 / 2}} \cdot \frac{\mu_{0} M_{s} H_{0}}{3 \cdot\left(75 k_{B} T_{0}\right)^{2}} \simeq 7.41 \cdot 10^{22} \mathrm{~m}^{-3}
$$

where $M_{s} \simeq M^{5 \mathrm{~T}}=184 \mathrm{kA} / \mathrm{m}$ (see table 6.10), or similarly $\rho=3 x /\left(4 \pi r^{3}\right)$, where $x$ is their volume fraction $(\approx 16 \%)$. Thus, the strong pinning observed for the $\mathrm{Nd}_{60} \mathrm{Fe}_{30} \mathrm{Al}_{10}$ "master alloy" samples are likely due to pinning centers whose radius is approximately

$$
r=\left(\frac{3 x}{4 \pi \rho}\right)^{1 / 3} \simeq 8 \mathrm{~nm}
$$

This result suggests therefore that even small differences of the microstructure may strongly influences the hard magnetic behavior of this alloy if the magnetic properties of the amorphous matrix and of the interface cluster/matrix do not vary significantly.

\subsubsection{Summary}

On the basis of the WAXS results, it may be concluded that the cooling rate with which the $\mathrm{Nd}_{60} \mathrm{Fe}_{30} \mathrm{Al}_{10}$ "master alloy" has been prepared does not influence the formation of the nanocrystalline phase and the samples result to be intrinsic composites consisting of nanocrystalline clusters whose mean size and volume fraction do not differ significantly from the values found for the mold-cast bulk specimens. Nevertheless, the thermodynamic behavior and the magnetic properties of the master alloy are not comparable to those of 
the bulk samples and differences of the thermal stability and of the hard magnetic behavior have been determined. In particular it has been possible to show that the Gaunt formalism accounting for strong domain-wall pinning determines the temperature dependence of the coercivity and the values of the parameters which define the pinning mechanism strongly differ from those found for the bulk sample.

This result seems therefore to indicate that even small difference of the morphology and size of the nanocrystalline precipitates may influence the magnetic behavior of the sample, confirming that cooling rate dependent magnetic properties can be obtained in the $\mathrm{Nd}_{60} \mathrm{Fe}_{30} \mathrm{Al}_{10}$ alloy.

\subsection{Splat-cooled Foil}

Beyond the slow-cooled "master alloy" and bulk samples, aim of this work has also been the investigation of rapid-quenched specimens which have been prepared by splatquenching (see section 2.2.1.4 with cooling rate of about $10^{6} \mathrm{~K} / \mathrm{s}$. This value is 4 orders of magnitude larger than that of the bulk sample.

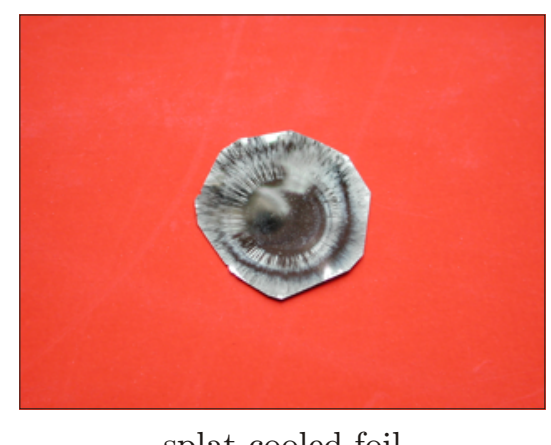

splat-cooled foil

Figure 6.39. Picture of a splat-cooled foil with a thickness of $30 \mu \mathrm{m}$ and a diameter of about $15 \mathrm{~mm}$.

Splat-cooled samples are thin foils with a thickness of about $30 \mu \mathrm{m}$ and $15 \mathrm{~mm}$ in diameter (see figure 6.39). For their characterization, the specimens have been cut in $5 \times 5 \mathrm{~mm}^{2}$ squares taken from the center of a splat sample.

\subsubsection{X-Ray Diffraction and Transmission Electron Microscopy}

In order to compare the structural properties of the slow- and rapid-quenched samples and to determine the role of the preparation method on their microstructure, splatcooled foils have been investigated by HE-WAXS, HRTEM and SANS following the same characterization procedure adopted for the bulk sample.

The 2-D x-ray diffraction pattern of an as-prepared sample measured at the 1-ID synchrotron beamline at the APS in Argonne is shown in figure 6.40. The measurement 


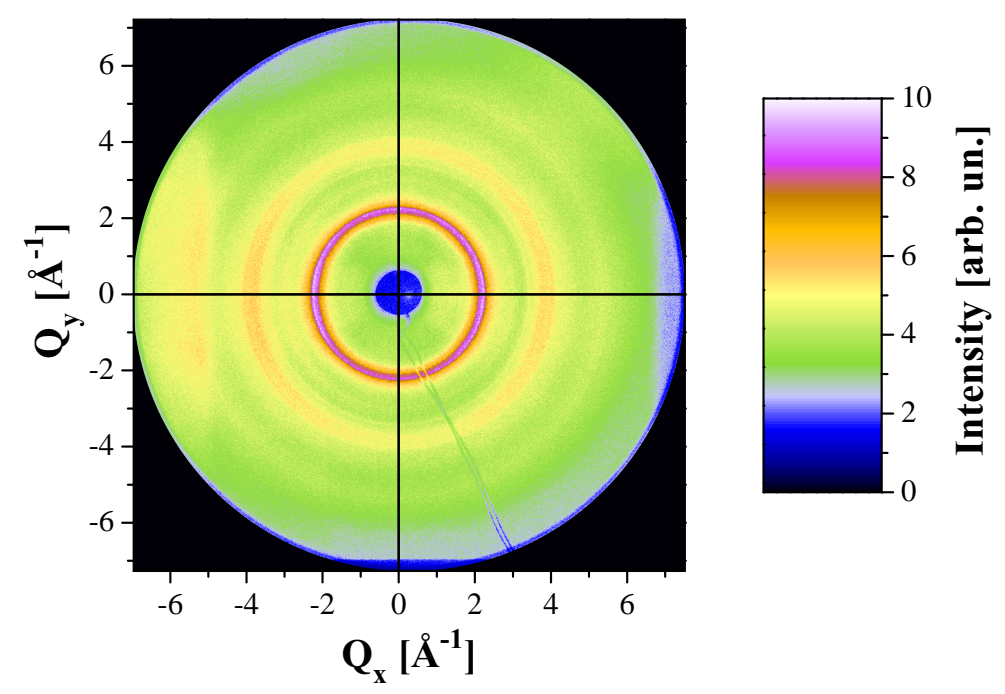

Figure 6.40. $2-\mathrm{D}$ high-energy diffraction pattern of a splat-quenched $\mathrm{Nd}_{60} \mathrm{Fe}_{30} \mathrm{Al}_{10}$ foil recorded at $301 \mathrm{~K}$.

has been recorded at $301 \mathrm{~K}$ with a collimated $\mathrm{x}$-ray beam with a transverse size of about $50 \times 50 \mu \mathrm{m}^{2}$. Figure 6.40 clearly shows that the splats are not fully amorphous although a relatively high cooling rate has been used for their preparation. Instead they are intrinsic composites consistent of random oriented crystals embedded in an amorphous matrix as the sharp Debye rings and the broad halos demonstrate.

The analysis of the one-dimensional curve which results from the angular averaging of figure 6.40 is shown in figure 6.41. The spectrum of the diffracted intensity vs. the scattering vector $Q$ is presented together with the curve measured for the bulk sample. The splat-cooled foils are clearly composed of a glassy matrix embedding a nanocrystalline phase whose volume fraction is much lower than that arising from the bulk samples, as the weak reflections demonstrate. The indexing of diffraction peaks by fitting the $Q$-values with equation 5.79 (see the right graph in figure 6.41) indicates that the ordered phase is a dhcp structure with lattice constants $a=(3.65 \pm 0.03) \AA$ and $c=(11.56 \pm 0.22) \AA$. The good accordance between these values and those found for the bulk and master alloy samples, and for metallic Neodymium, confirms that the crystalline phase is Nd-rich. The low intensity of the diffraction peaks cannot permit however to estimate the mean size of the crystalline grains which has been for this reason investigated by transmission electron microscopy.

Figure 6.42 (left) shows a conical dark-field TEM image and a selected-area electron diffraction (SAD) pattern of an as-prepared splat which demonstrates the presence of randomly oriented nanocrystals and of an amorphous matrix (see also the high-resolution image on the right of figure 6.42). The chemical analysis performed in-situ by EDX indicates that the nanocrystals have a Nd concentration larger than $80 \%$ while the composition of the matrix do not differ significantly from the nominal $\mathrm{Nd}_{60} \mathrm{Fe}_{30} \mathrm{Al}_{10}$. The SAD 

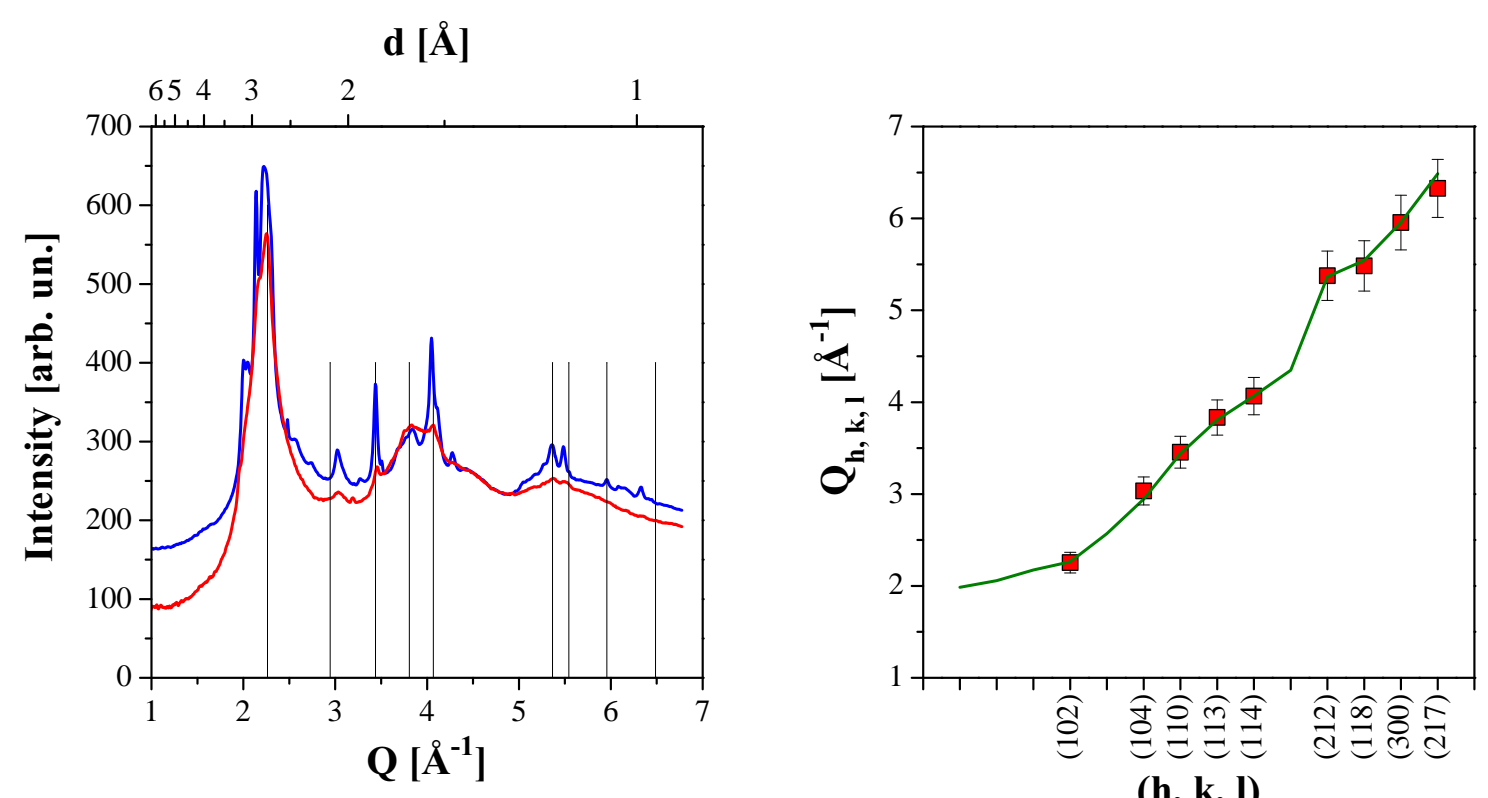

Figure 6.41. (Left) Diffraction intensity vs. scattering vector $Q$ of a $\mathrm{Nd}_{60} \mathrm{Fe}_{30} \mathrm{Al}_{10}$ splatcooled foil (-) and of a bulk sample (-) obtained by azimuthal averaging of 2-D HE-WAXS measurements recorded at $301 \mathrm{~K}$. The vertical lines $(-)$ represent the main reflections arising from a dhcp structure of lattice parameters $a=(3.65 \pm 0.03) \AA$ and $c=(11.56 \pm$ $0.22) \AA$. (Right) $Q$-values of the diffraction peaks visible in the left graph ( $\square)$ and the fit according to equation 5.79 (-).
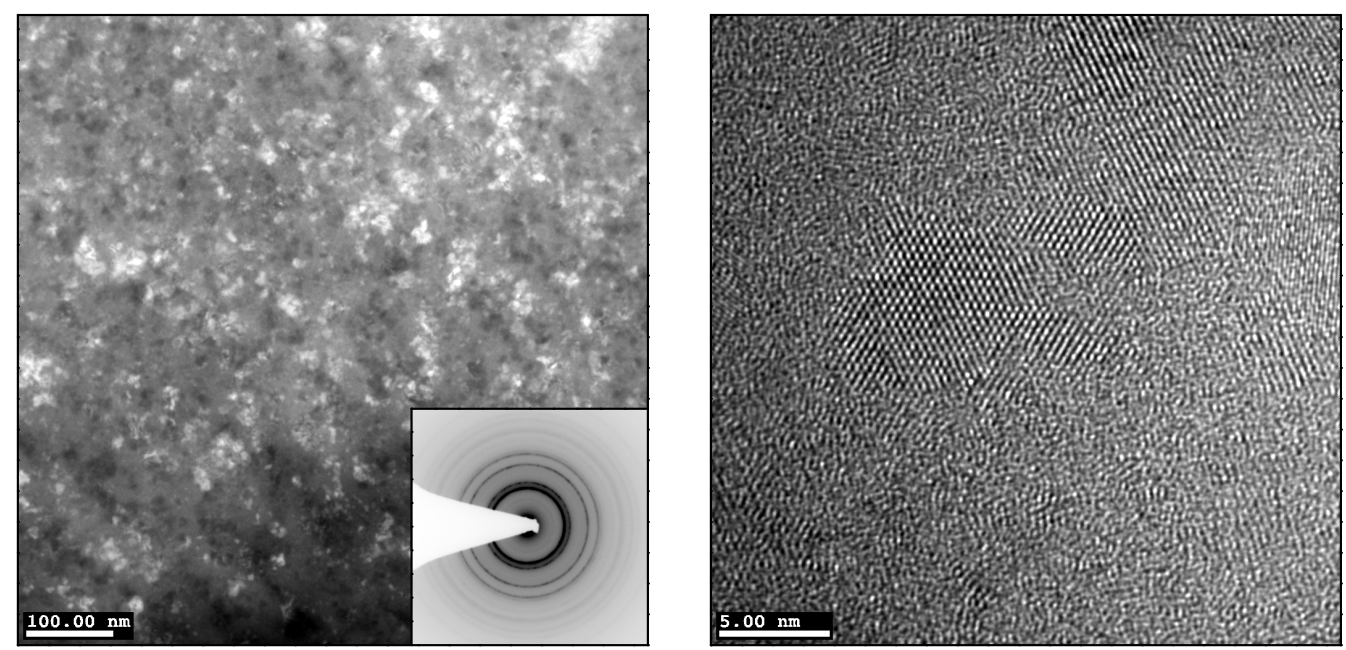

Figure 6.42. (Left) Conical dark-field image and selected-area electron diffraction (SAD) picture of a $\mathrm{Nd}_{60} \mathrm{Fe}_{30} \mathrm{Al}_{10}$ splat foil. The TEM picture shows nanocrystalline agglomerates (bright and dark areas) embedded in a glassy phase (gray areas). (Right) High-resolution image of a Nd-rich nanocrystal. 
indicates, however, that the nanocrystals are ordered in a bixbyite structure suggesting the presence of $\mathrm{Nd}_{2} \mathrm{O}_{3}$ and confirming the oxidation of the $\mathrm{Nd}-$ rich phase upon sample thinning which has been also observed in the bulk specimens. Nevertheless, assuming that the mean size of the $\mathrm{Nd}$-oxide grains cannot be smaller than that of the in-situ formed Nd-rich nanocrystals, the TEM images of the splat foils permit to determine that their mean lateral dimension is about $t=(10 \pm 3) \mathrm{nm}$.

In accordance with the results of the bulk and master alloy samples, the experimental data of the rapid-quenched splats suggests that the Nd-rich crystals form during the solidification process as consequence of phase separation in the melt or in the undercooled liquid. The observed decrease of the nanocrystals size and the reduced volume fraction which can be deduced from the $\mathrm{x}$-ray data seems also to suggest that the increased cooling rate may hamper the nucleation-and-growth process of this phase but not prevent the phase separation.

\subsubsection{Differential Scanning Calorimetry and Thermal Stability}

In analogy to the analysis on bulk and master alloy samples, the splat-cooled foils have been investigated by differential scanning calorimetry to determine the thermal stability of the amorphous phase upon annealing, and the influence of the cooling rate on the thermodynamic properties. If the metastable state of the system differs from that of the previous specimens, strong differences of the calorimetric data should be expected.

The thermal stability of $\mathrm{Nd}_{60} \mathrm{Fe}_{30} \mathrm{Al}_{10}$ splats has been determined by DSC measurements and by analyzing the shift of the crystallization temperature upon increase of the heating rate via the Kissinger method (see equation 3.27). Several DSC curves have been therefore recorded employing heating rates between 1 and $80 \mathrm{~K} / \mathrm{min}$. The consequent Kissinger plot is shown in figure 6.43 together with the experimental data relative to the bulk sample. The linear regression permits to obtain the values of the two unknown parameters $\Delta E_{c}$ and $K_{0}$ which are listed in table 6.12 .

\begin{tabular}{cccc}
\hline \multirow{2}{*}{ Parameter } & Splat Foil & Value & \\
& Bulk & Master Alloy \\
\hline$\Delta E_{c}$ & $(1.87 \pm 0.01) \mathrm{eV}$ & $(2.21 \pm 0.01) \mathrm{eV}$ & $(2.72 \pm 0.01) \mathrm{eV}$ \\
$K_{0}$ & $(5.26 \pm 0.16) \cdot 10^{10} \mathrm{~s}^{-1}$ & $(1.65 \pm 0.15) \cdot 10^{12} \mathrm{~s}^{-1}$ & $(1.51 \pm 0.06) \cdot 10^{15} \mathrm{~s}^{-1}$ \\
\hline
\end{tabular}

Table 6.12. Parameters deduced from the Kissinger analysis on $\mathrm{Nd}_{60} \mathrm{Fe}_{30} \mathrm{Al}_{10}$ splat-cooled foils according to equation 3.27 . The correspondent values for the bulk sample and the "master alloy" are shown for comparison.

The activation energy and the frequency factor of the splat foils result to be lower than those of the bulk samples indicating a reduced thermal stability against crystallization. Due to relations which link $\Delta E_{c}$ and $K_{0}$ to the kinematic and thermodynamic 


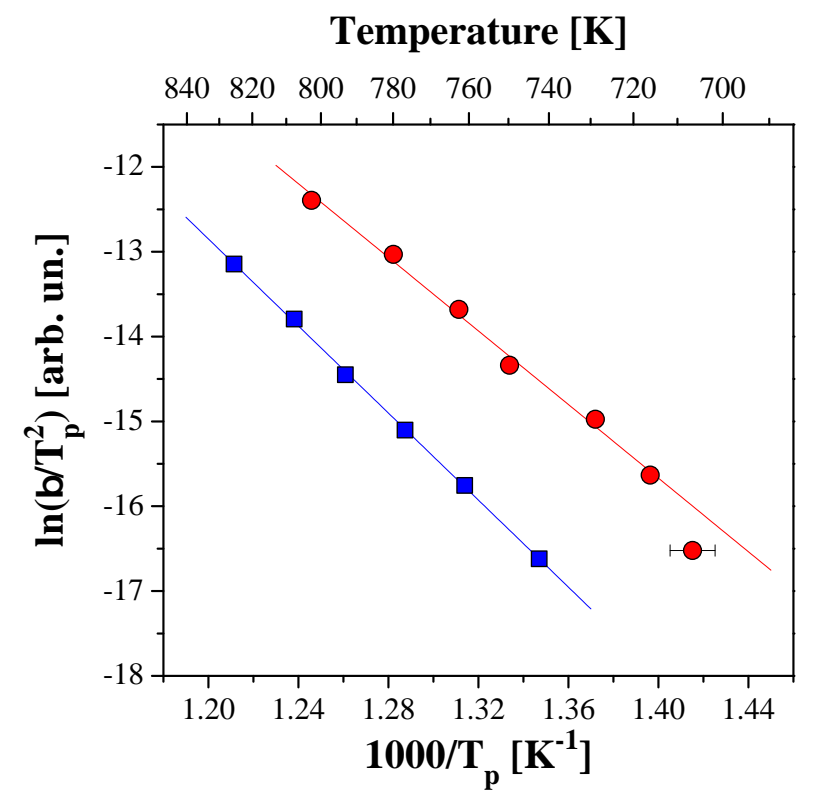

Figure 6.43. Kissinger plots of $\mathrm{Nd}_{60} \mathrm{Fe}_{30} \mathrm{Al}_{10}$ splat-cooled ( $\mathrm{O}$ ) and bulk ( $\boldsymbol{\square}$ ) samples. The lines represents the fits according to equation 3.27 .

properties of a metastable system (see section 3.1.1), and their dependence from the chemical composition, the differences arising from the Kissinger analysis indicate that the amorphous phase of the splats differs significantly from that of the bulk samples. The

\section{Time [year]}

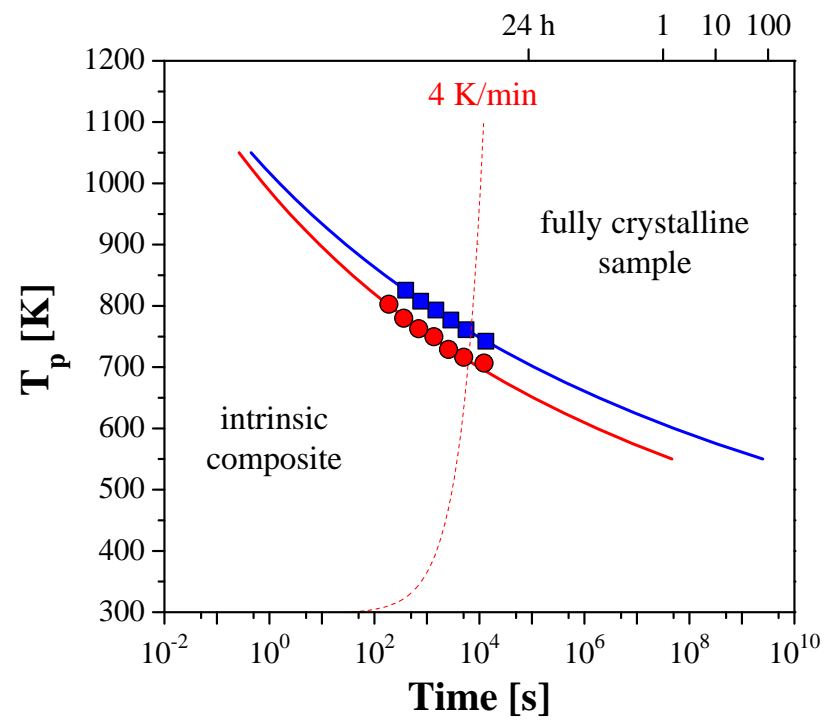

Figure 6.44. Transformation diagram for a $\mathrm{Nd}_{60} \mathrm{Fe}_{30} \mathrm{Al}_{10}$ splat (-) and bulk sample (-) as derived from equation 3.27 . The symbols indicate the relative crystallization temperatures $T_{p}^{\beta}$ which have been measured at different heating rates $\beta$.

different thermodynamic behavior of the rapid-quenched samples emerges clearly from the continuous heating transformation diagram (see section 3.1 .2 shown in figure 6.44 
and obtained from the values of $\Delta E_{c}$ and $K_{0}$. The graph confirms the differences between slow- and rapid-quenched $\mathrm{Nd}_{60} \mathrm{Fe}_{30} \mathrm{Al}_{10}$ specimens which emerged from the structural analysis and, furthermore, it evidences as the consequences of any thermal treatment on the microstructural properties may differ significantly from one sample to the other.

\subsubsection{AC and DC Magnetometry}

The results of the structural properties of the splat-cooled foils have shown a complex microstructure whose features differ from those of the bulk and master alloy samples. In order to determine similarities and differences on their magnetic behavior the rapidquenched material has been investigated by AC and DC magnetometry.

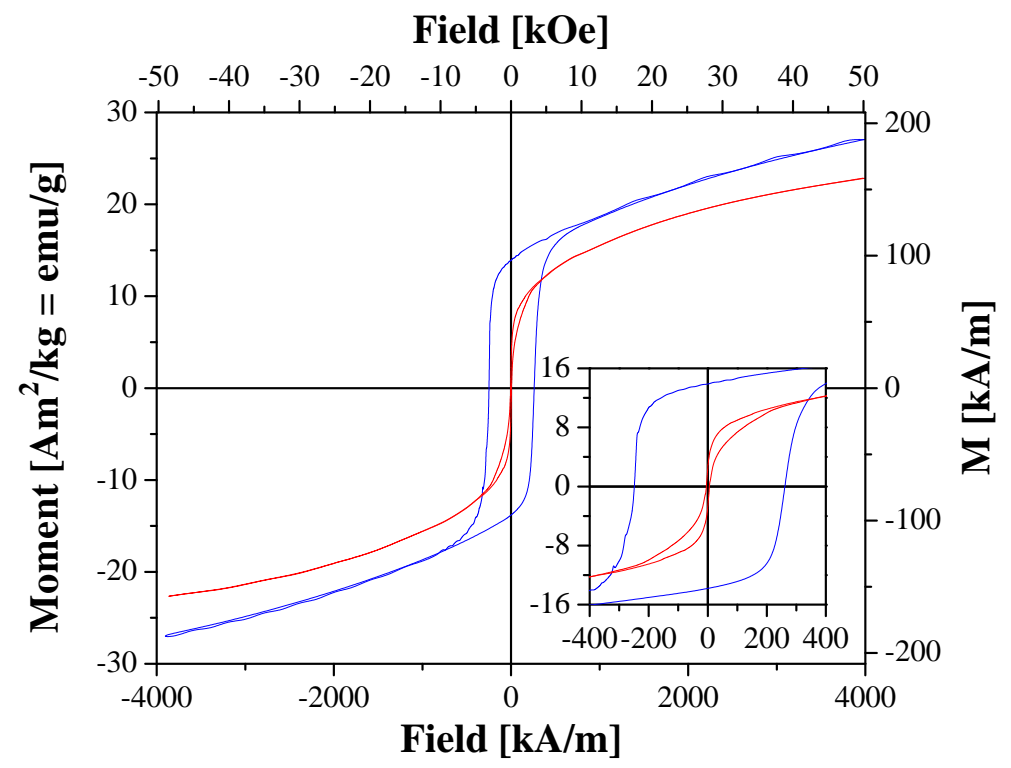

Figure 6.45. Hysteresis loops of a $\mathrm{Nd}_{60} \mathrm{Fe}_{30} \mathrm{Al}_{10}$ rapid-quenched foil (-) and of a bulk sample $(-)$ recorded at $300 \mathrm{~K}$.

Figure 6.45 shows two hysteresis loops recorded at $300 \mathrm{~K}$ from a splat-quenched foil and a bulk sample, respectively. The rapid-quenched specimen has a reduced net magnetization and small values of the remanence and of the coercive field (see table 6.13). In particular, the coercivity decrease from the value of $246 \mathrm{kA} / \mathrm{m}$ measured for the bulk sample to about $5 \mathrm{kA} / \mathrm{m}(\approx 63 \mathrm{Oe})$ indicating a strong decrease of $H_{c}$ with an increasing cooling rate. High coercivities and a monotonic decrease of the $H_{c}$ values with an increase in the cooling rate have also been observed in rapidly quenched $\mathrm{Nd}_{60} \mathrm{Fe}_{30} \mathrm{Al}_{10}$ and $\mathrm{Nd}_{60} \mathrm{Fe}_{x} \mathrm{Co}_{30-x} \mathrm{Al}_{10}$ 100,101, melt-spun $\mathrm{Nd}-\mathrm{Fe}$ ribbons and other rare-earth transition metal amorphous alloys 43 .

Although a coercive field of $5 \mathrm{kA} / \mathrm{m}$ is about 30 times smaller than the value measured for the bulk sample, the splat-cooled sample shows still hard-magnetic behavior according to the criterion proposed by Hernando and coworkers [5]. Low values of the coercive 


\begin{tabular}{cccc}
\hline \multirow{2}{*}{ Parameter } & \multicolumn{3}{c}{ Value } \\
& Splat Foil & Bulk & Master Alloy \\
\hline$M^{5 \mathrm{~T}}$ & $159 \mathrm{kA} / \mathrm{m}$ & $183 \mathrm{kA} / \mathrm{m}$ & $184 \mathrm{kA} / \mathrm{m}$ \\
$M_{r}$ & $17 \mathrm{kA} / \mathrm{m}$ & $93 \mathrm{kA} / \mathrm{m}$ & $76 \mathrm{kA} / \mathrm{m}$ \\
$H_{c}$ & $5 \mathrm{kA} / \mathrm{m}$ & $246 \mathrm{kA} / \mathrm{m}$ & $142 \mathrm{kA} / \mathrm{m}$ \\
$\left(\mu_{0} M \cdot H\right)_{\max }$ & $26 \mathrm{~J} / \mathrm{m}^{3}$ & $19 \mathrm{~kJ} / \mathrm{m}^{3}$ & $4 \mathrm{~kJ} / \mathrm{m}^{3}$ \\
\hline
\end{tabular}

Table 6.13. Values of the magnetization at $5 \mathrm{~T} M^{5 \mathrm{~T}}$, of the remanence $M_{r}$, of the coercivity $H_{c}$, and of the product $\left(\mu_{0} M \cdot H\right)_{\max }$ measured at $300 \mathrm{~K}$ for a $\mathrm{Nd}_{60} \mathrm{Fe}_{30} \mathrm{Al}_{10}$ splat-cooled foil. The correspondent values for the bulk sample and the "master alloy" are shown for comparison.

field are commonly expected in fully amorphous systems since the effective anisotropy is averaged out over a large magnetic correlated volume. However, the high field which seems to be necessary to saturate the rapid-quenched sample may be an indication that noncollinear magnetic order may play an important role in the magnetization mechanisms of the $\mathrm{Nd}_{60} \mathrm{Fe}_{30} \mathrm{Al}_{10}$ alloy and hence, on the basis of the random anisotropy model, it may be supposed that the effective anisotropy of this system remains relatively large (small correlated volumes) and determines the high coercive field.

Nevertheless, the structural investigations have indicated that the splat-cooled foils are heterogeneous materials and their magnetic characterization is of fundamental importance to understand the effects governing the coercivity mechanisms of those samples.

Figure 6.46 shows a field-cooled thermomagnetic curve recorded with a magnetic field $\mu_{0} H=1 \mathrm{~T}$ in the temperature range from 1.8 to $550 \mathrm{~K}$. Two ferromagnetic-to-

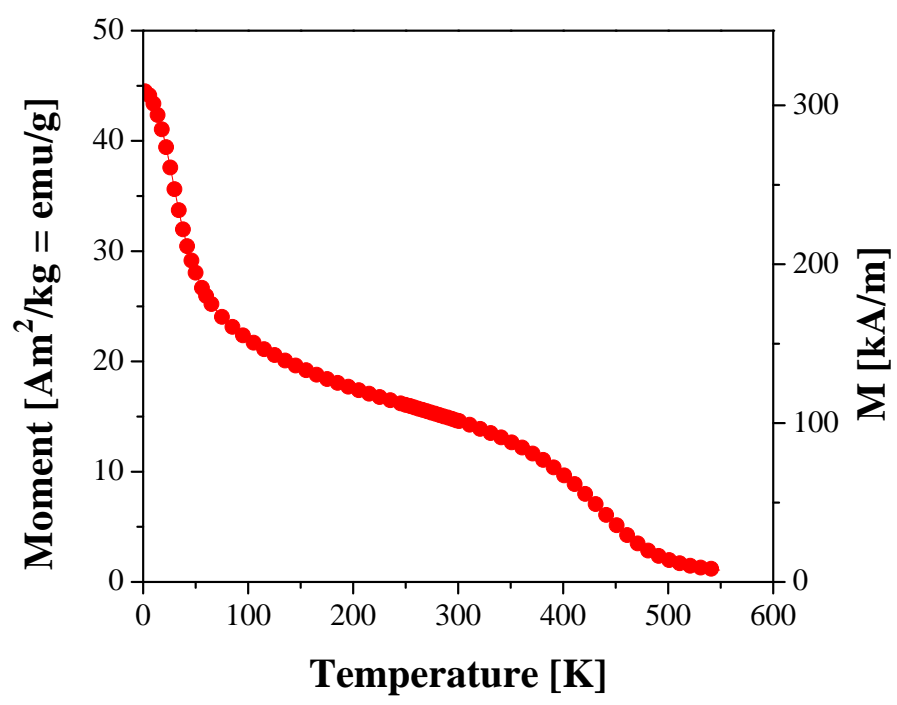

Figure 6.46. Field-cooled thermomagnetic curve of an as-prepared splat foil measured in the range from 1.8 to $550 \mathrm{~K}$ with an applied magnetic field of $1 \mathrm{~T}$. 
paramagnetic transitions take place at about 50 and $500 \mathrm{~K}$, respectively, indicating the presence of two different magnetic phases. In accordance with the results of the bulk samples, these results suggest that the high ordering temperature is associated with the Fe-rich amorphous matrix while the crystalline $\mathrm{Nd}$-rich phase is responsible for the low$T_{C}$ transition.

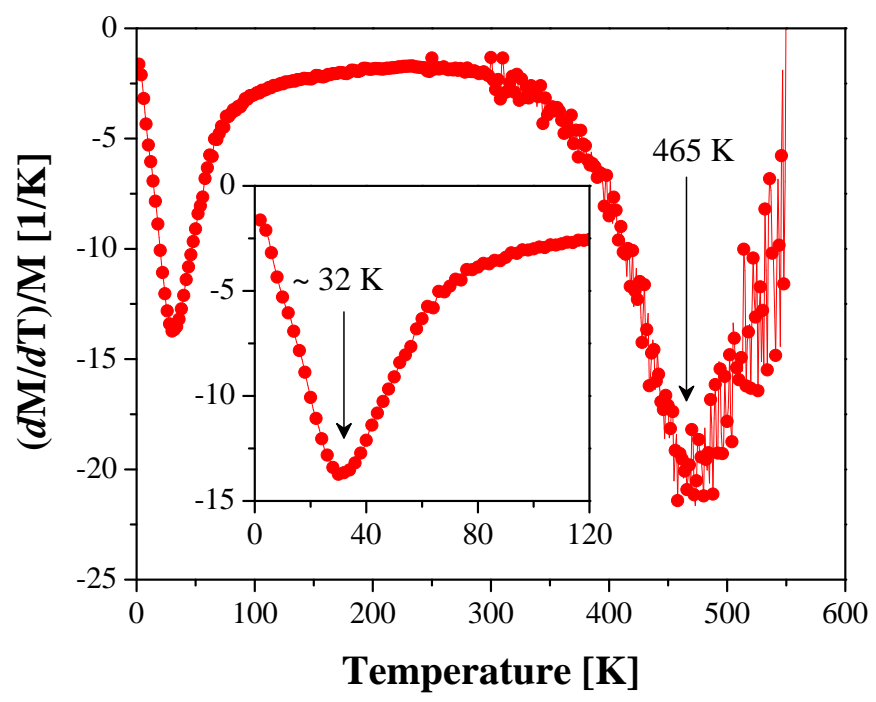

Figure 6.47. Normalized derivative $(d M / d T) / M$ vs. $T$ calculated from the FC thermomagnetic scan shown in figure 6.46 .

The normalized temperature derivative of the magnetization $(d M / d T) / M$, which is shown in figure 6.47, confirms this hypothesis but it also indicates a reduction of the Curie temperature of the high- $T_{C}$ phase and strong differences in the low-temperature magnetic behavior. The broad minimum in the $(d M / d T) / M$ vs. $T$ curve of the bulk sample becomes a well defined peak (at about $32 \mathrm{~K}$ ) for the splat foils. Therefore, in order to determine the differences between the two samples, the real $\left(\chi^{\prime}\right)$ and the imaginary $\left(\chi^{\prime \prime}\right)$ part of the AC susceptibility has been measured as a function of the temperature between 2 and $300 \mathrm{~K}$. As shown in figure 6.48, $\chi^{\prime}(T)$ and $\chi^{\prime \prime}(T)$ present a complex temperature dependence and the presence of several peaks which can likely be associated to different magnetic transitions. In particular, $\chi^{\prime \prime}(T)$ presents two small maxima at 10 and $30 \mathrm{~K}$, in good accordance with the results on the bulk sample, and an increase at about $50 \mathrm{~K}$ which has not been observed before. The broad peak whose onset is at about $200 \mathrm{~K}$ may be associated to the high-temperature transition of the amorphous matrix. These results suggest that, below $80 \mathrm{~K}$, the splat-cooled foils are characterized by at least two magnetic phases whose contributions to the sample net magnetization remain however difficult to determine (see for comparison figure 6.46 and figure 6.47).

A second interesting feature of the rapid-quenched samples emerges from the measurements of the hysteresis loops at and below room temperature. Figure 6.49 shows the virgin magnetization curves measured at 120 (left) and $300 \mathrm{~K}$ (right), respectively. At 


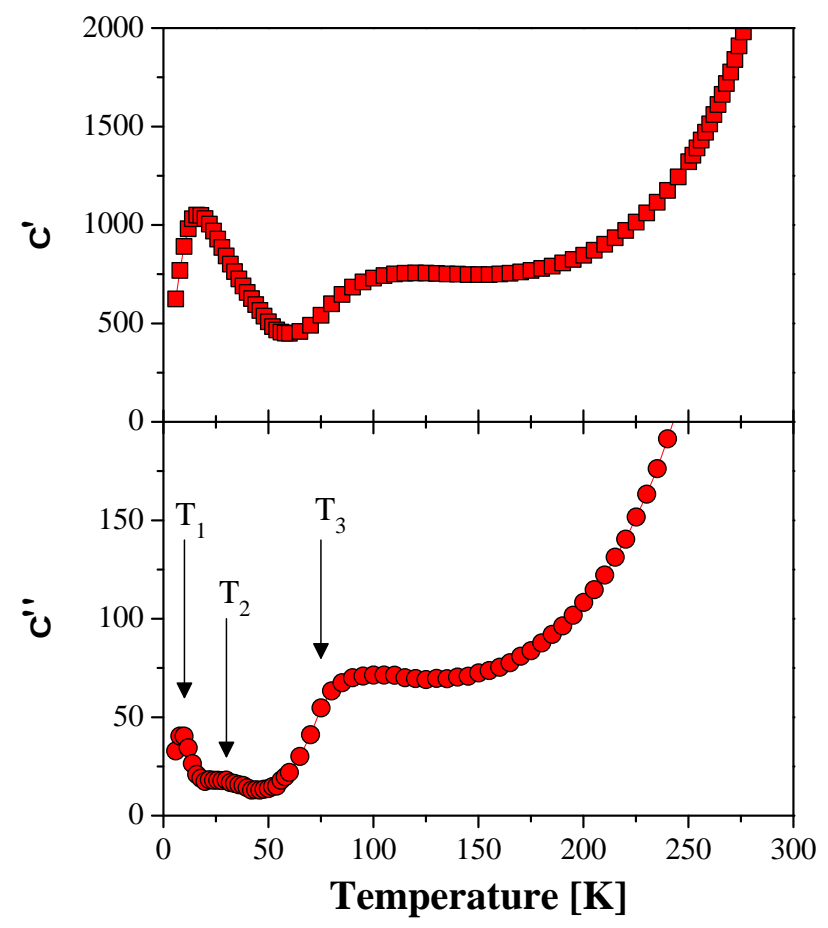

Figure 6.48. Real (top) and imaginary (bottom) AC susceptibility of a splat-cooled sample measured with a SQUID magnetometer in the temperature range from 5 to $300 \mathrm{~K}$ (driving field: 4 Oe, wave frequency: $117.04 \mathrm{~Hz}$ ).

Field [kOe]

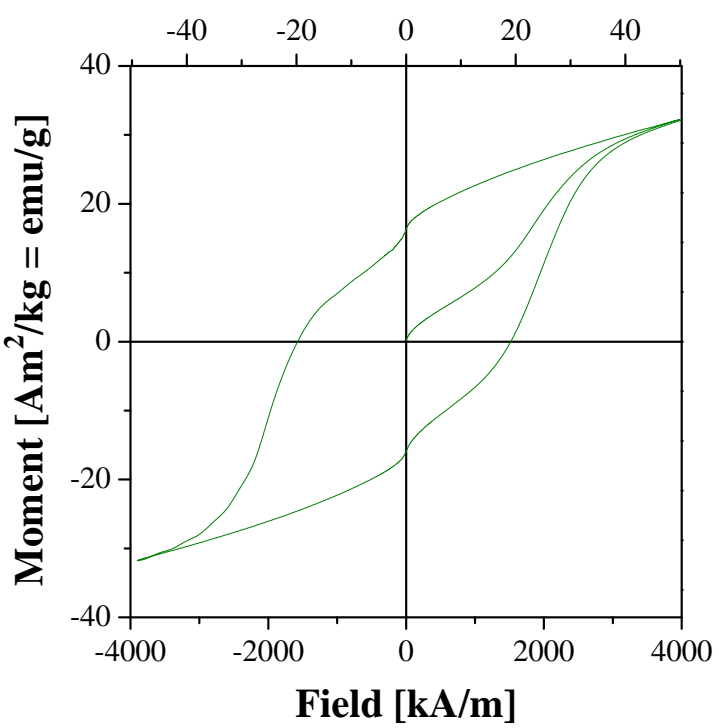

Field [kOe]

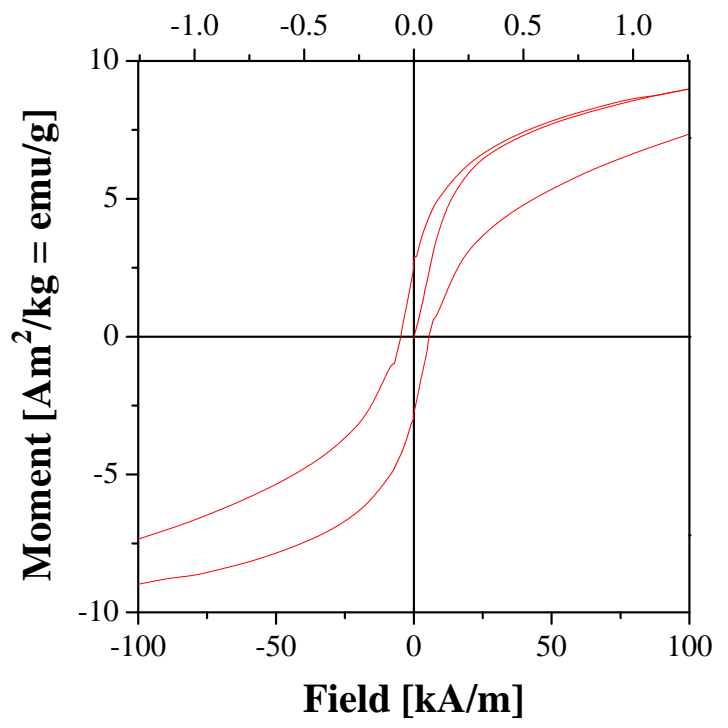

Figure 6.49. (Left) Initial magnetization curves of a $\mathrm{Nd}_{60} \mathrm{Fe}_{30} \mathrm{Al}_{10}$ splat-cooled foil measured at $120 \mathrm{~K}(-)$. Note the nucleation-type magnetization followed by a pinning-type behavior. (Right) The same curve measured at $300 \mathrm{~K}(-)$. 
room temperature, the hysteresis loop has a typical nucleation-type behavior (see section 4.4 which is characterized by a strong variation of the magnetization as the applied field increases. At low temperature, the shape of loop changes dramatically and shows a kink at low field which is commonly observed in a two-phases system and a singular virgin curve which presents an initial nucleation-like behavior followed by a pinning-type one at higher values of the magnetic field. Between 40 and $120 \mathrm{~K}$ the hysteresis loops do not change significantly and the coercivity remains almost constant. As the temperature decreases below $40 \mathrm{~K}$, the maximal magnetization at $5 \mathrm{~T}$ strongly increases, the shape of the loops change in accordance with the results of the bulk samples and the coercivity decreases.

These evidences suggest that a further magnetic phase must be responsible for the appearance of the kink at low magnetic field below room temperature. According to the results of Sato Turtelli [162], the rapid quenching methods commonly employed for the preparation of $\mathrm{Nd}_{60} \mathrm{Fe}_{30} \mathrm{Al}_{10}$ melt-spun ribbons and splat-cooled foils are responsible for inevitable temperature gradients between the inner and the outer part of the samples. This determines local differences of the cooling rate along the sample thickness and the formation of a soft-magnetic phase on the side of the specimen which comes in contact with the cold copper surfaces (see section 2.2.1.4). This conclusion has been experimentally supported by measuring the hysteresis loops after the removal of few $\mu \mathrm{m}$ of the sample surface by mechanical polishing [163]. The curves obtained for temperatures higher than $50 \mathrm{~K}$ do not show any kink at zero field, while the low-temperature features remain unchanged as well as the Curie temperature of the amorphous matrix. Furthermore, the difference of the coercive field between 120 and $300 \mathrm{~K}$ does not change significantly after the $e x-$ situ treatment.

These results indicate that the magnetic properties of the splat-cooled sample are strongly influenced by the low-temperature phase(s) and only marginally by the soft phase ordering around room temperature. The interplay between the low- $T c$ crystalline phase and the ferromagnetic amorphous phase is likely responsible for the singular transition from nucleation- to pinning-type behavior which has been shown in figure 6.49. Furthermore, the appearance of pinning effects below $300 \mathrm{~K}$ suggests that the temperature dependence of the coercive field may be understood in terms of the Gaunt model (see section 6.3.6).

\subsubsection{Small-Angle Neutron Scattering}

On the basis of these results, small angle neutron scattering (SANS) has been employed to investigate the chemical and magnetic homogeneity of the splat-cooled samples. Measurements in zero magnetic field and in a maximum magnetic field of $1.5 \mathrm{~T}$ have been performed to separate nuclear and magnetic scattering contributions which may arise from 
spatial variations of nuclear and magnetic neutron contrasts, respectively.
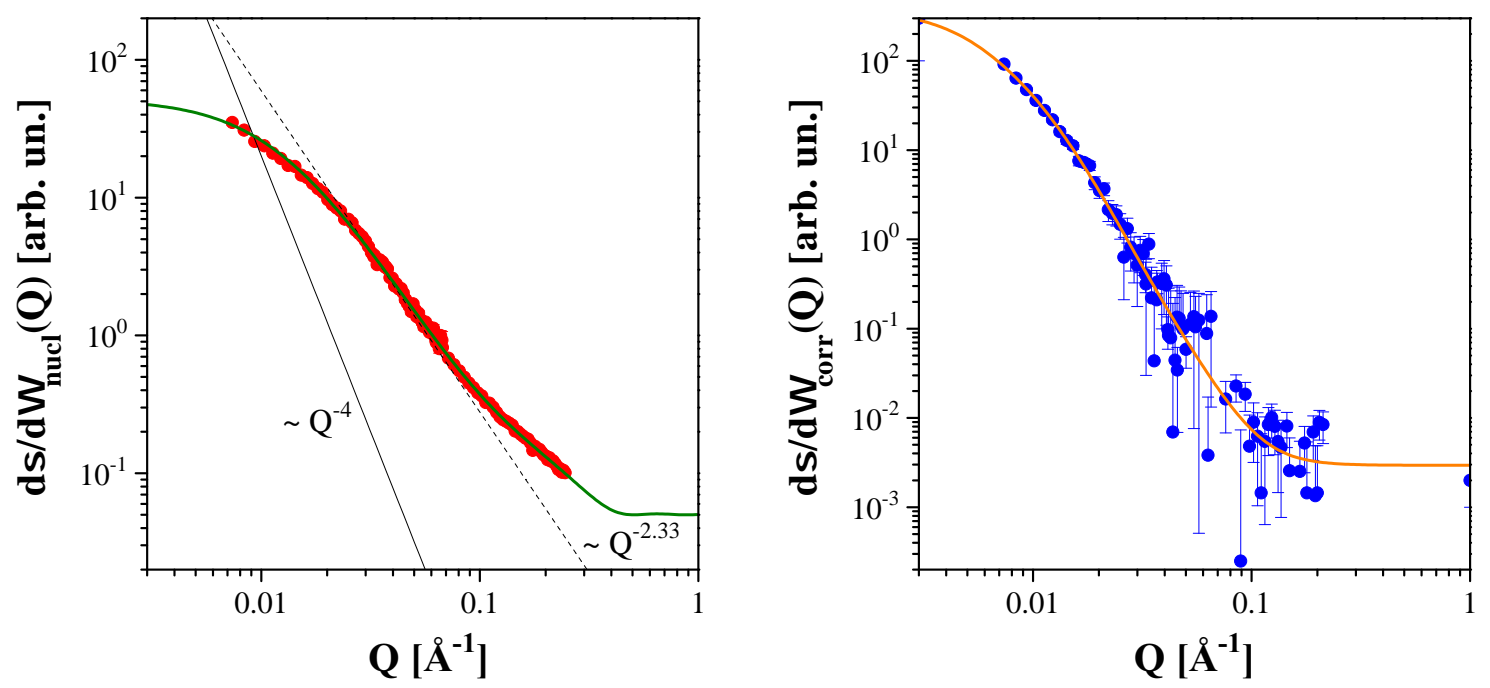

Figure 6.50. (Left) Nuclear cross-section of a $\mathrm{Nd}_{60} \mathrm{Fe}_{30} \mathrm{Al}_{10}$ splat-cooled sample measured at room temperature in an external magnetic field of $1.5 \mathrm{~T}$. The quantitative analysis of the SANS data has been described in section 5.3.4. The fit (-) is in accordance to equation C.11. (Right) Magnetic scattering curve deduced from the SANS data of the splat sample measured with and without magnetic field. The fit $(-)$ is in accordance to equation B.4.

SANS measurements with and without magnetic field and a sector analysis of the relative scattering patterns allow to calculate the mean size of precipitates, with scattering length density which differs from that of the matrix, and to evaluate the magnetic correlation length of the magnetic moments in a ferromagnetic system [106. Following the quantitative analysis of the SANS described in section 5.3.4 the scattering curves due to the nuclear and magnetic contrasts have been obtained. The nuclear cross-section which is presented in figure 6.50 (left) exhibits a power-law scattering $I(Q) \propto Q^{-\alpha} 17$ indicating a scattering process due to a mass fractal network formed by aggregation of clusters whose scattering length density differs from that of the embedding matrix [62]. The difference in the chemical composition between the clusters and the matrix is responsible for the measured nuclear scattering contrast. In order to determine the structural aspects of the fractal network, the nuclear cross-section has been fitted by equation C.11 and the three parameters describing a mass fractal object: the mass fractal dimension $d_{f}$, the cluster size $r_{0}$, and the mean lateral dimension $\xi$ have been obtained [19]. Their values are listed in table 6.14 together with the parameters $B$ and $P$ expressing the background signal and the scattering factor, respectively (see equation C.11).

On the contrary the magnetic cross-section has been calculated by the data reduction described in section 5.3 .4 and by equation 5.56. The averaged magnetic correlation length has been evaluated by fitting the relative magnetic scattering curve shown in figure 6.50 (right) and assuming an exponentially decaying correlation with crossover-length 


\begin{tabular}{ccc}
\hline \multirow{2}{*}{ Parameter } & \multicolumn{2}{c}{ Value } \\
& Splat Foil & Bulk \\
\hline$B$ & $0.054 \pm 0.002$ & $0.078 \pm 0.003$ \\
$P$ & $0.0328 \pm 0.0006$ & $0.07 \pm 0.01$ \\
$r_{0}$ & $(5.2 \pm 0.4) \AA$ & $(5.2 \pm 0.4) \AA$ \\
$d_{f}$ & $2.3 \pm 0.02$ & $2.53 \pm 0.01$ \\
$\xi$ & $(87 \pm 4) \AA$ & $(188 \pm 21) \AA$ \\
\hline
\end{tabular}

Table 6.14. Fit parameters according to equation C.11 and to the SANS curve shown in figure 6.50 (left) of a $\mathrm{Nd}_{60} \mathrm{Fe}_{30} \mathrm{Al}_{10}$ splat-cooled foil.

$R$ [47]. The fit of the data according to equation B.4 permits to obtain a mean spatial magnetic correlation length of $L_{\text {corr }}=2 R=(34 \pm 6) \mathrm{nm}$ [17, 18

These results indicate in analogy with the evaluation on the bulk samples that the $\mathrm{Nd}$-rich precipitates have a complex morphology which can be described by a mass fractal distribution $M \propto\left(r / r_{0}\right)^{d_{f}}$ (equation 6.3). The value $d_{f}=2.3$ suggests, moreover, that the high cooling rate of the splat sample influences the mass distribution of the fractalclusters which shows therefore a smaller mass fractal dimension and a reduced density (see section C) if compared to the bulk samples.

The evaluation of the magnetic correlation length gives a value of only $34 \mathrm{~nm}$ which is much lower than the estimation proposed for the bulk sample $\left(L_{c o r r}>150 \mathrm{~nm}\right)$ indicating that the ferromagnetic order may be strongly affected by random anisotropy and that a smaller correlated volume is energetically favorable than large ferromagnetic domains (see section 4.3.1. The importance of this result will be discussed in section 6.3.6 considering the coercivity mechanisms and the possible origin of the high coercive field measured in the splat-cooled foils.

\subsubsection{Mössbauer Spectroscopy}

In order to confirm the results of the structural and magnetic investigation which suggest the presence of two phases with different Fe concentrations, ${ }^{57} \mathrm{Fe}$ Mössbauer spectroscopy has been employed to define the Fe distribution between paramagnetic (or superparamagnetic) and ferromagnetic sites.

Results of the Mössbauer spectroscopy taken at room temperature and zero magnetic field on a $\mathrm{Nd}_{60} \mathrm{Fe}_{30} \mathrm{Al}_{10}$ splat foil are shown in figure 6.51. The considerable broadening of the lines is typical for amorphous alloys. The experimental spectrum can be fitted well with a superposition of (super-)paramagnetic and ferromagnetic contributions. The fit indicates that $65 \%$ of the site population may be attributed to ferromagnetic sites and only $35 \%$ to (super-)paramagnetic ones. This result is in good agreement with the magnetic measurement indicating two magnetic phases and suggests that the phase with 


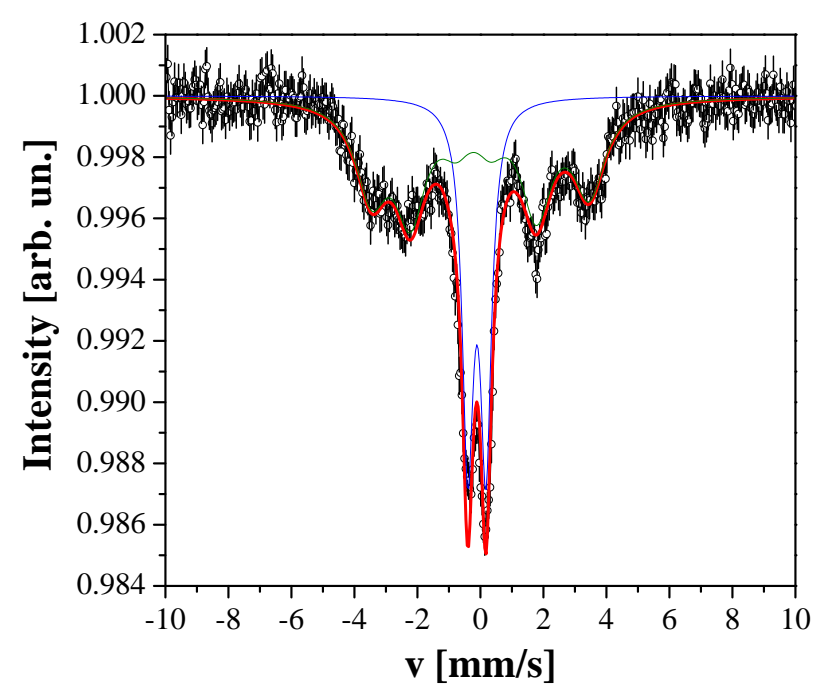

Figure 6.51. ${ }^{57} \mathrm{Fe}$ Mössbauer spectrum of $\mathrm{Nd}_{60} \mathrm{Fe}_{30} \mathrm{Al}_{10}$ rapid-quenched splat foil taken at room temperature in zero magnetic field. The line $(-)$ is the sum of a (super-)paramagnetic $(-)$ and a ferromagnetic (-) contributions which result from the fitting procedure.

lower magnetic ordering temperature has a small concentration of Fe compared to that with higher $T_{C}$ which can be then assumed to be Fe-rich |18|. Furthermore the number of ferromagnetic Fe sites in this phase is lower than that found in the bulk samples $(\sim 84 \%)$. Such differences may account not only for the different magnetic behavior but also for the reduced thermal stability shown by the splats.

\subsubsection{Coercivity Mechanisms}

According to the previous results, the splat-cooled samples show a more complex magnetic behavior than the bulk sample or the master alloy. In fact, although the low temperature magnetic properties are similar for all samples due to the influence of the magnetic transition of the crystalline phase, the splat foils show a singular behavior with a typical pinning-type virgin curve up to room temperature and nucleation-type loops above $300 \mathrm{~K}$. In order to understand the complex magnetization processes and the coercivity mechanisms of the splat samples, several hysteresis loops have been measured by SQUID and VSM magnetometry between 2 and $500 \mathrm{~K}$ in order to determine the temperature dependence of the coercive field. Figure 6.52 (left) shows the variation of the coercivity which has been deduced from the hysteresis curves. The $H_{c}$ value of $5 \mathrm{kA} / \mathrm{m}$ at $T=300 \mathrm{~K}$ increases steeply as the temperature decreases, and reaches a maximum value of $1964 \mathrm{kA} / \mathrm{m}(\approx 24.7 \mathrm{kOe})$ at $100 \mathrm{~K}$ before decreasing again at lower temperature. Considering the behaviors of the previous samples and assuming that the interplay of the two phases in the temperature range where one of them is paramagnetic determines pinning effects of the domain walls of the other phase, the strong pinning formalism of Gaunt has 

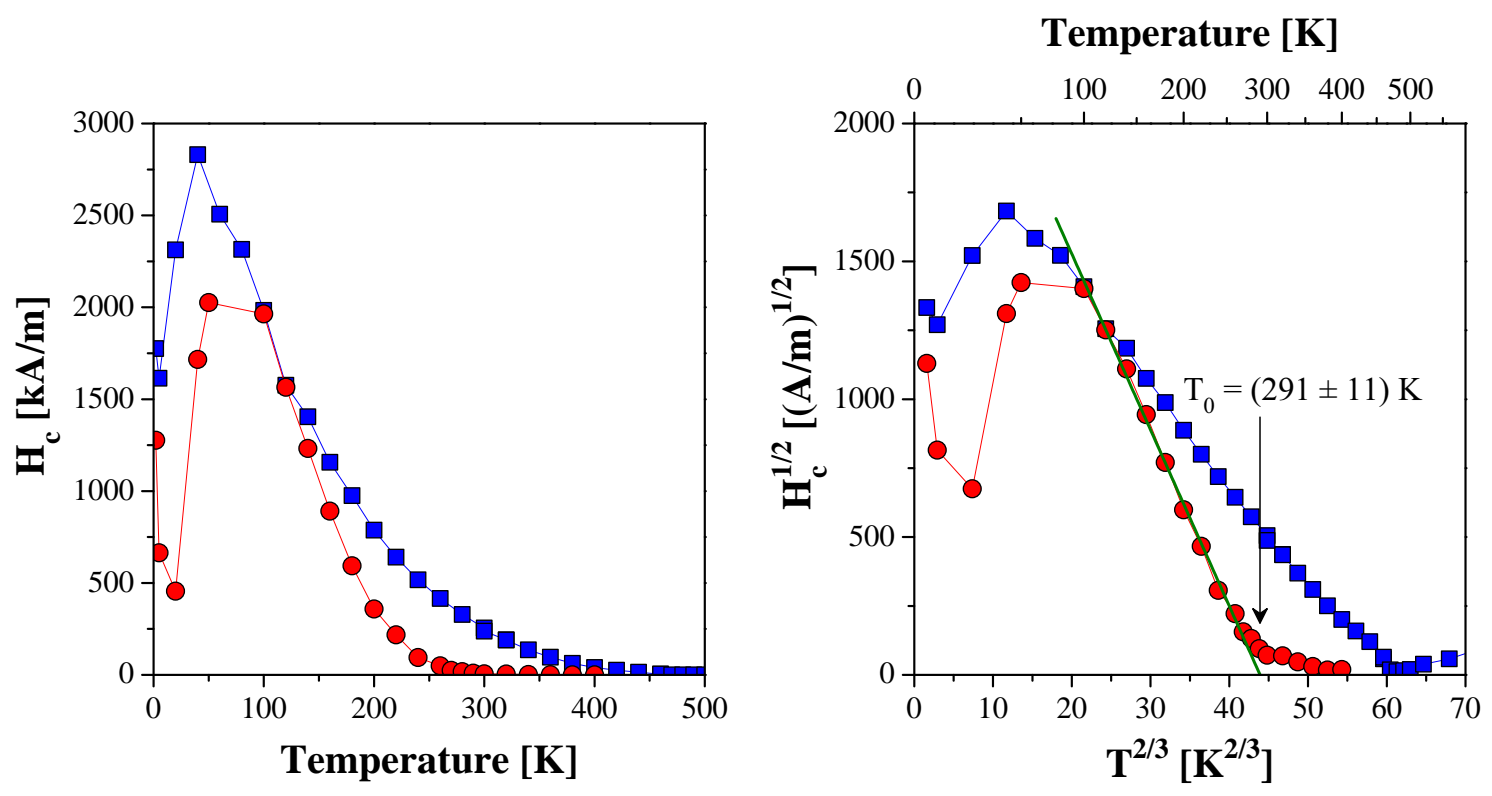

Figure 6.52. (Left) Temperature dependence of the coercivity field of a $\mathrm{Nd}_{60} \mathrm{Fe}_{30} \mathrm{Al}_{10}$ splatcooled sample in the temperature range from 2 to $500 \mathrm{~K}(\mathrm{O})$. (Right) Gaunt plot $H_{c}^{1 / 2}$ vs. $T^{2 / 3}$ of the experimental data shown on the left graph and, for comparison, the curve obtained for the bulk sample ( $\square$ ) (see figure 6.17). The line is the linear fit between 100 and $280 \mathrm{~K}$ according to equation 4.53 .

been considered.

Figure 6.52 (right) shows the Gaunt plot $H_{c}^{1 / 2}$ vs. $T^{2 / 3}$ deduced from the experimental data from 2 to $500 \mathrm{~K}$. The Gaunt equation is in good agreement with the experimental data in the temperature range from 100 to about $280 \mathrm{~K}$ and the linear fit allows to obtain the parameters $H_{0}$ and $T_{0}$ of equation 4.53. Their values are reported in table 6.15 [17.

\begin{tabular}{ccc}
\hline \multirow{2}{*}{ Parameter } & \multicolumn{3}{c}{ Value } \\
& Splat Foil & Bulk \\
\hline$H_{0}$ & $(7866 \pm 177) \mathrm{kA} / \mathrm{m}$ & $(4625 \pm 67) \mathrm{kA} / \mathrm{m}$ \\
$T_{0}$ & $(291 \pm 11) \mathrm{K}$ & $(451 \pm 12) \mathrm{K}$ \\
\hline
\end{tabular}

Table 6.15. Intrinsic coercive field $H_{0}$ and the crossover temperature $T_{0}$ as obtained by fitting the experimental data $H_{c}^{1 / 2}$ vs. $T^{2 / 3}$ of figure 6.52 (right) according to the domain wall pinning model of Gaunt 63 . (see equation 4.53).

The high value of the intrinsic coercive field $H_{0}$ obtained from the fitting of the Gaunt's plot is consistent with previous results on amorphous $\mathrm{Nd}_{40} \mathrm{Fe}_{60}$ 126 which show lowtemperature $H_{c}=6287 \mathrm{kA} / \mathrm{m}$, and the low $T_{0}$ indicates that the pinning mechanisms are already overcome by thermal fluctuation at room temperature. This implies therefore that the morphology of the pinning sites and the variation of the magnetic properties at the interface with the amorphous matrix may differ significantly from those of the bulk 
sample. In analogy to the analysis proposed in section 6.1.4, pinning effects are strong if

$$
\frac{A^{3 / 2}}{K^{1 / 2}} \ll \frac{225 k_{B} T_{0}}{4 \pi^{4}}=2.32 \cdot 10^{-21}
$$

considering $A^{3 / 2} / K^{1 / 2}=6.4 \cdot 10^{-23} \mathrm{~J}$ (such that the ratio between $A^{3 / 2} / K^{1 / 2}$ and $225 k_{B} T_{0} / 4 \pi^{4}$ remains equal to that assumed for the bulk sample) and according to equation 6.11 , the density of pinning sites becomes

$$
\rho=8 \pi^{4} \frac{A^{3 / 2}}{K^{1 / 2}} \cdot \frac{\mu_{0} M_{s} H_{0}}{3 \cdot\left(75 k_{B} T_{0}\right)^{2}} \simeq 2.9 \cdot 10^{23} \mathrm{~m}^{-3}
$$

where $M_{s} \simeq M^{5 \mathrm{~T}}=159 \mathrm{kA} / \mathrm{m}$ (see table 6.13). This value indicates that the pinning density in rapid-quenched $\mathrm{Nd}_{60} \mathrm{Fe}_{30} \mathrm{Al}_{10}$ is lower than in slow-cooled samples and, assuming on the basis of the $\mathrm{x}$-ray data that the volume fraction is roughly $x=5 \%$ and that $\rho=3 x /\left(4 \pi r^{3}\right)$, the observed strong pinning is likely due to pinning centers whose radius is approximately

$$
r=\left(\frac{3 x}{4 \pi \rho}\right)^{1 / 3} \simeq 3.5 \mathrm{~nm}
$$

This value is less than half of that obtained by SANS measurements of the nuclear contrast ( $\xi=9 \mathrm{~nm}$ ), therefore pSANS has been employed to verify the reliability of the core-shell model (introduced in section 6.1.8) in the case of the splat-cooled samples.

Figure 6.53 shows the pSANS signals measured on a splat foil at room temperature for the polarization states $I^{+}$and $I^{-}$, the relative sum $\left(I^{-}+I^{+}\right)$and difference signals $\left(I^{-}-I^{+}\right.$) (see section 5.3.5). While the sum signal is isotropic as the pattern for nonpolarized neutrons, the difference signal is strongly anisotropic with the expected $\sin ^{2}(\alpha)$ behavior. Applying to the $2-\mathrm{D}$ patterns $\left(I^{-}+I^{+}\right)$and $\left(I^{-}-I^{+}\right)$the analysis and the angular averaging presented in section 5.3.5, the nuclear and the magnetic cross sections shown in figure 6.54 can be obtained. As expected, the nuclear signal is in good agreement with the previous SANS results as the accordance between the pSANS curve and the fit obtained by equation C.11 (using the values of $r_{0}, d_{f}$, and $\xi$ in table 6.14) clearly shows. The magnetic signal has been determined by fitting the curve in figure 6.54 (right) with equation C.11 and with the values of the parameters $B, P, r_{0}, d_{f}$, and $\xi$ listed in table 6.16 $\left(R^{2}=0.976\right)$.

The value $\xi$ is in good agreement with the previous calculation on the effective pin radius $(3.6 \mathrm{~nm})$ and suggests that the core-shell model can be applied to the splat-cooled sample to interpret both the results of the Gaunt analysis and of the small angle scattering.

The domain wall pinning processes is determined by the complex core-shell structure of the Nd-rich nanocrystals whose mean size $\xi_{\text {nucl }} \simeq 9 \mathrm{~nm}$ has been determined by SANS, and which have a paramagnetic "nucleus" of radius $\xi_{\text {magn }} \simeq 4 \mathrm{~nm}$ and a magnetic shell of thickness $\left(\xi_{\text {nucl }}-\xi_{\text {magn }}\right) \simeq 5 \mathrm{~nm}$.

These results seem to explain the nature of the pinning mechanisms and the high values of the coercivity of the samples at low temperature. However, the process which 

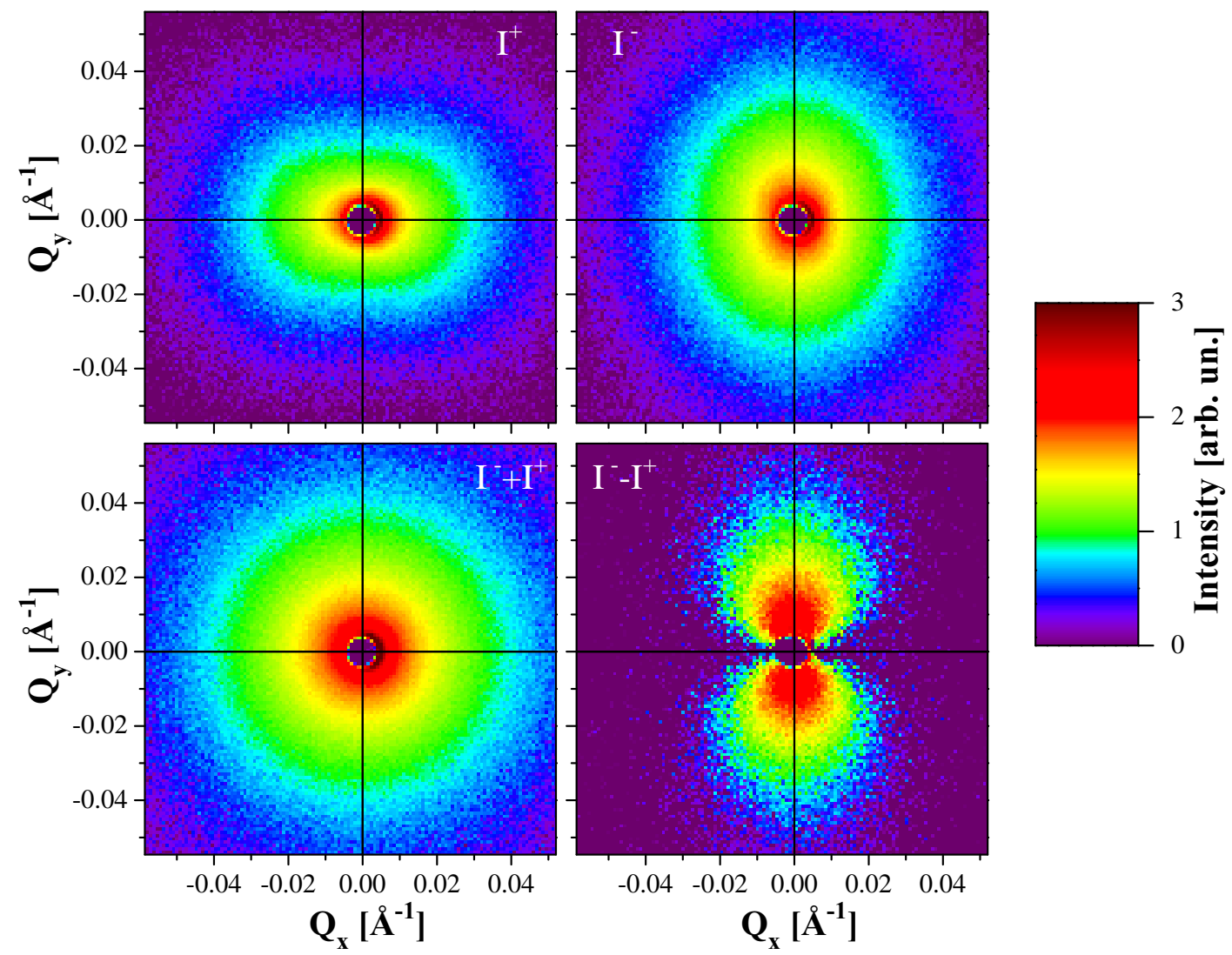

Figure 6.53. Small angle neutrons scattering patterns for the two polarization states $I^{+}$ and $I^{-}$, for the relative sum $\left(I^{-}+I^{+}\right)$and difference signals $\left(I^{-}-I^{+}\right)$measured on a splat-cooled sample at room temperature.

\begin{tabular}{ccc}
\hline \multirow{2}{*}{ Parameter } & \multicolumn{2}{c}{ Value } \\
& Splat Foil & Bulk \\
\hline$B$ & $0.145 \pm 0.014$ & $0.025 \pm 0.006$ \\
$P$ & $0.011 \pm 0.001$ & $0.0089 \pm 0.0008$ \\
$r_{0}$ & $(5.0 \pm 0.4) \AA$ & $(4.0 \pm 0.4) \AA$ \\
$d_{f}$ & $2.91 \pm 0.07$ & $2.51 \pm 0.04$ \\
$\xi$ & $(41 \pm 2) \AA$ & $(65 \pm 4) \AA$ \\
\hline
\end{tabular}

Table 6.16. Parameters of equation C.11 obtained by fitting the magnetic pSANS cross section shown in figure 6.54 (right). 

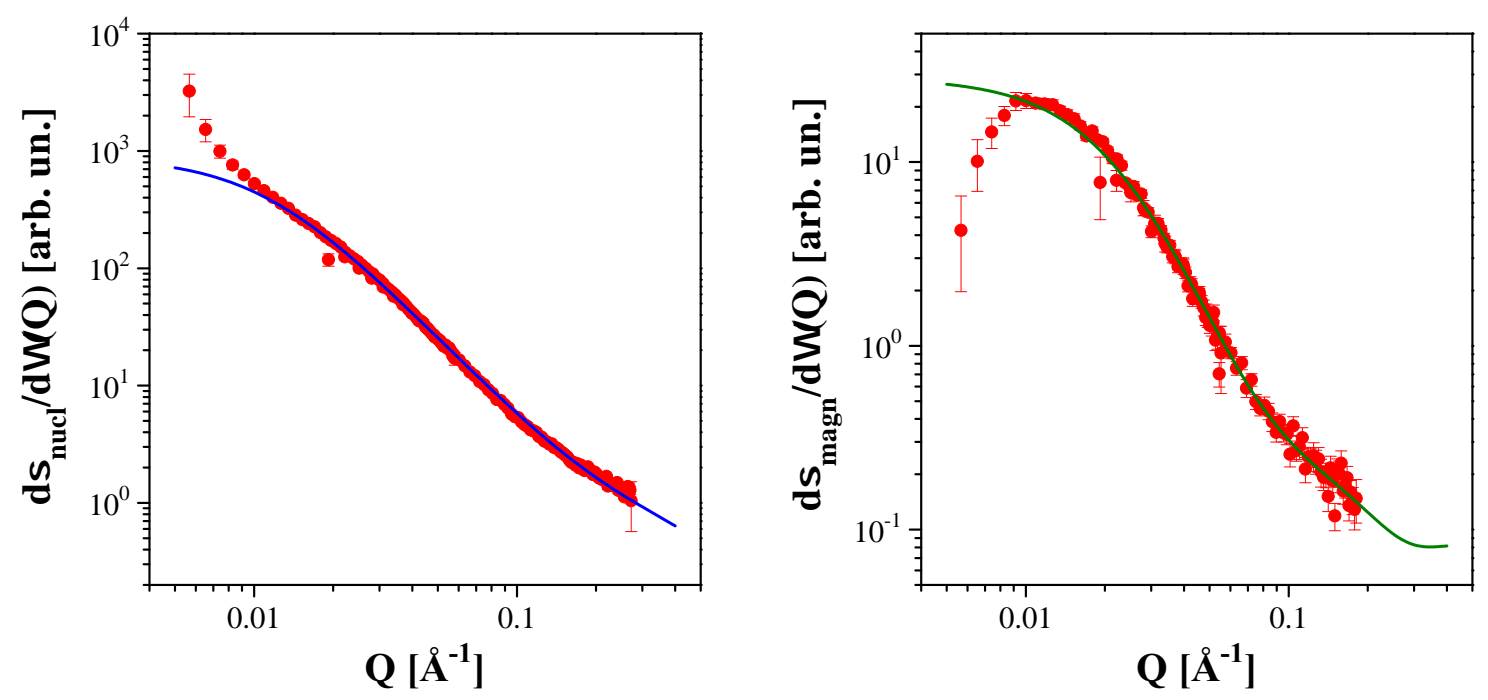

Figure 6.54. Reconstructed nuclear (left) and magnetic (right) cross sections obtained by averaging the $2-\mathrm{D}$ pSANS patterns $\left(I^{-}+I^{+}\right)$and $\left(I^{-}-I^{+}\right)$according to the analysis proposed in section 5.3.5. The line $(-)$ is the fit of the SANS curve of figure 6.50 according to equation C.11 and the parameters listed in table 6.14. The line $(-)$ represents instead the fit of the pSANS magnetic cross sections according to equation C.11 and the parameters listed in table 6.16.

determines the coercivity at room temperature when, for $T>T_{0}$, the pinning forces are averaged out by thermal fluctuations, is still unclear. Experimentally, the data in figure 6.52 deviate considerably from the Gaunt's fit around and above the characteristic temperature $T_{0}=291 \mathrm{~K}$. Therefore, to explain the magnetic properties of the splat foils three different temperature ranges must be distinguished. Above $T_{0}$, the domain walls do not experience any impediments from the pinning centers and the alloy's coercive field depends only on the intrinsic magnetization mechanism of the ferromagnetic phase. According to the random anisotropy model (see section 4.3), the coercivity of an amorphous system has its origin in the value of the effective anisotropy energy, which is given by averaging the local anisotropy in the exchange coupled volume $\left(\approx L_{e x}^{3}\right)$. The local magnetic anisotropy of the amorphous ferromagnetic Fe-rich phase is strongly affected by the large magnetocrystalline anisotropy of $\mathrm{Nd}$ atoms. Hence, the effective anisotropy acting over the correlation volume $\sim(34 \mathrm{~nm})^{3}$ may explain the relatively high coercivity $(5 \mathrm{kA} / \mathrm{m})$ shown by the $\mathrm{Nd}_{60} \mathrm{Fe}_{30} \mathrm{Al}_{10}$ splat at room temperature. As $T$ falls below $T_{0}$ (i.e., $240 \mathrm{~K}=T^{*}<T<T_{0}$ ), the domain-wall pinning process starts to influence the magnetic properties of the sample but, being the thermal energy only slightly lower than the pinning energy barrier $\left(T^{*} / T_{0}=0.86\right)$, the values of the coercivity are determined by the interplay between random anisotropy and pinning mechanisms. Below $T^{*}$, the thermal energy is much lower than the pinning activation energy, thereby increasing the effect of the pinning process and, consequently, the magnetic field necessary to break away the domain walls from the pinning sites. 


\subsubsection{Summary}

The results of the rapidly quenched $\mathrm{Nd}_{60} \mathrm{Fe}_{30} \mathrm{Al}_{10}$ foils confirm the tendency of this alloy to undergo a phase separation which results in the formation of Nd-rich nanocrystals embedded in an amorphous phase. Phase separation in the undercooled liquid may be responsible for the complex microstructure which is characterized by mass fractal aggregates whose chemical composition differs from that of the remnant matrix. Compared to the bulk sample, splat foils have a smaller crystalline volume fraction and slightly reduced grain size suggesting that the higher cooling rates employed for the solidification of these samples may effectively control the nucleation and the growth of the stable Nd-rich phase.

Although the low-temperature magnetic investigation has shown several magnetic transitions, only one dhcp crystalline phase has been clearly identified by $\mathrm{x}$-ray diffraction and HRTEM. Local EDX analysis has confirmed that the nanocrystalline precipitates are Nd-rich. On the contrary, it has not been possible to exclude fluctuation or variation of the chemical composition in the amorphous phase which would suggest the presence of other phases.

Such presence has been however indicated by AC and DC magnetometry. In the temperature range $80 \mathrm{~K}<T<300 \mathrm{~K}$, where only the amorphous matrix results to be ferromagnetic, pinning effects have been indicated to be responsible for the observed coercivity mechanisms. The Gaunt model has indicated that the temperature variation of the coercive field is due to strong low-dense pinning centers with a mean radius of only $4 \mathrm{~nm}$. This value has been effectively measured by pSANS confirming the importance of the core-shell model introduced to explain the pinning behavior of the slow-cooled samples.

The height of the energy barriers determined by the pinning centers (i.e., the strength of the pinning sites) is however lower for the splat foils than for the bulk specimens and thermal fluctuations are able at room temperature to overcome them. This behavior has been confirmed by measuring the initial magnetization curves below and above the Gaunt activation temperature $T_{0}(291 \mathrm{~K})$ and showing the transition from the pinningtype to nucleation-type loops. At $T>300 \mathrm{~K}$, due to the absence of other magnetic phases and of pinning effects, the system can be considered in good approximation as "homogeneous" amorphous magnet with high saturation field and, hence, non-collinear magnetic order. In this context, according to the random anisotropy model, the coercivity of an amorphous system has its origin in the value of the effective anisotropy energy, which is given by averaging the local anisotropy in the exchange coupled volume $L_{\text {corr }}^{3}$ where $L_{\text {corr }}$ is the correlation length. For the $\mathrm{Nd}_{60} \mathrm{Fe}_{30} \mathrm{Al}_{10}$ splat foils, the spontaneous magnetic correlation length has been found to be about $34 \mathrm{~nm}$ and, assuming that the local magnetic anisotropy of the amorphous ferromagnetic Fe-rich phase is affected by the large magnetocrystalline anisotropy of $\mathrm{Nd}$ atoms, the effective anisotropy acting over the correlation volume $(34 \mathrm{~nm})^{3}$ may explain the relatively high coercive field of $5 \mathrm{kA} / \mathrm{m}$ 
which has been recorded at $T=300 \mathrm{~K}$ 17.

In conclusion, it can be deduced that the coercivity of the splat-cooled samples is mainly controlled by domain wall pinning. However, the strength of this mechanism depends strongly on the temperature which determines when domain wall pinning dominates the intrinsic effects due to random anisotropy and vice versa. Hence, for $T<T_{0}=291 \mathrm{~K}$, $H_{c}$ is mainly due to pinning of the domain wall at core-shell sites, while for $T>T_{0}, H_{c}$ is controlled by the effective anisotropy of the ferromagnetic matrix.

\subsection{Thin Film}

The results on "master alloy" (cooling rate of some $\mathrm{K} / \mathrm{s}$ ), bulk samples (cooling rate of about $10^{2} \mathrm{~K} / \mathrm{s}$ ), and splat foils (quenched at a rate of about $10^{6} \mathrm{~K} / \mathrm{s}$ ) have proven the composite nature of the $\mathrm{Nd}_{60} \mathrm{Fe}_{30} \mathrm{Al}_{10}$ specimens and the strong interplay between the structural and magnetic features. This section deals with results on $\mathrm{Nd}_{60} \mathrm{Fe}_{30} \mathrm{Al}_{10}$ thin films prepared by a condensation method with cooling rates of the order of $10^{10} \mathrm{~K} / \mathrm{s}$ (see section 2.2.2). Thus the tendency of this alloy to form a glass is greatly enhanced allowing, likely, to redesign characteristic length scales and magnetization mechanisms which generally determine the properties of a magnetic system.

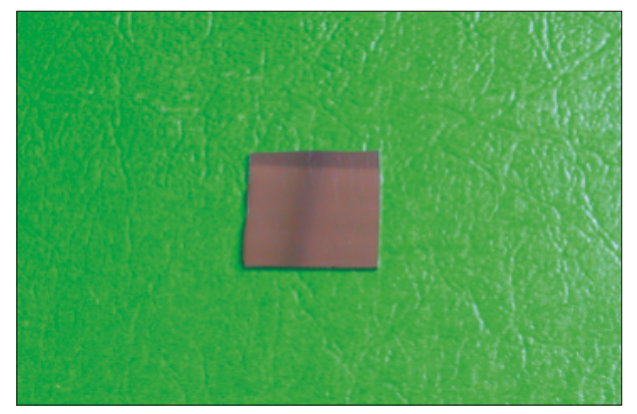

Figure 6.55. Picture of $\mathrm{Nd}_{60} \mathrm{Fe}_{30} \mathrm{Al}_{10}$ film deposited on a Si substrate.

$\mathrm{Nd}_{60} \mathrm{Fe}_{30} \mathrm{Al}_{10}$ thin films with a thickness of $50 \mathrm{~nm}$ have been characterized by $\mathrm{x}-$ ray diffraction and high-resolution transmission electron microscopy. The magnetic properties have been measured by SQUID magnetometry in the temperature range from 2 to $250 \mathrm{~K}$.

\subsubsection{X-Ray Diffraction and Transmission Electron Microscopy}

The $\mathrm{x}$-ray characterization of $\mathrm{Nd}_{60} \mathrm{Fe}_{30} \mathrm{Al}_{10}$ thin films has been carried out by employing a traditional laboratory diffractometer (Siemens D5000 with $\mathrm{Cu}-\mathrm{K}_{\alpha}$ radiation) and high-energy synchrotron $\mathrm{x}$-ray scattering (HE-WAXS). Both methods show a liquid-like pattern typical of amorphous samples and no Bragg reflections of a crystalline phase can be identified (see the 2-D WAXS pattern in figure 6.56 and figure 6.57). 


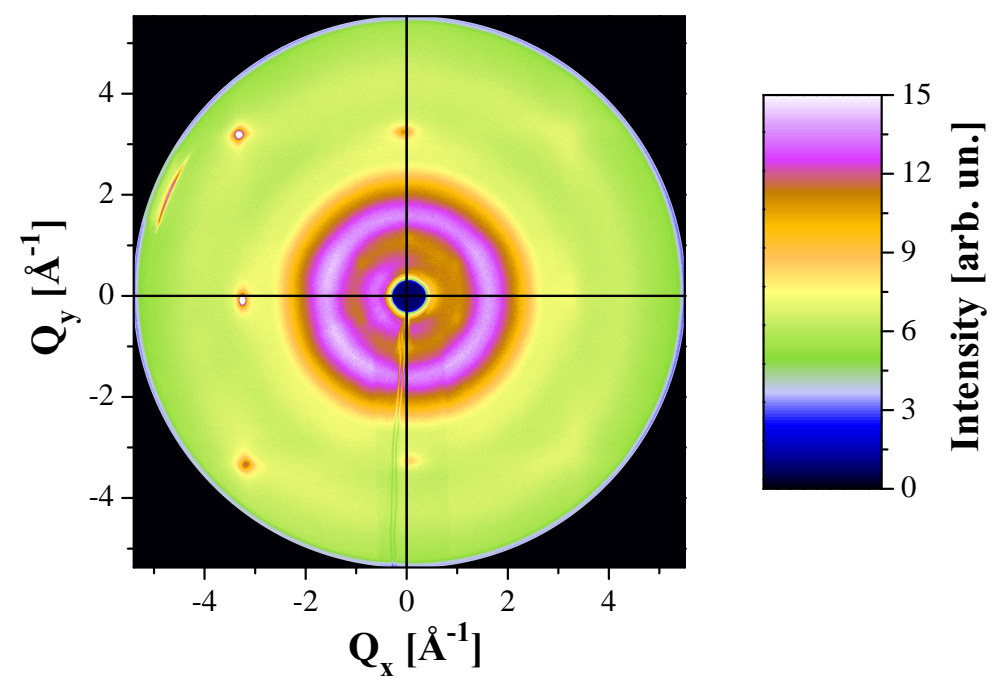

Figure 6.56. Diffuse WAXS pattern of $\mathrm{Nd}_{60} \mathrm{Fe}_{30} \mathrm{Al}_{10}$ thin film recorded with the incoming $\mathrm{x}$-ray beam perpendicular to the film plane. The sharp diffraction spots arise from the crystalline substrate while the sample results to be $\mathrm{x}$-ray amorphous.

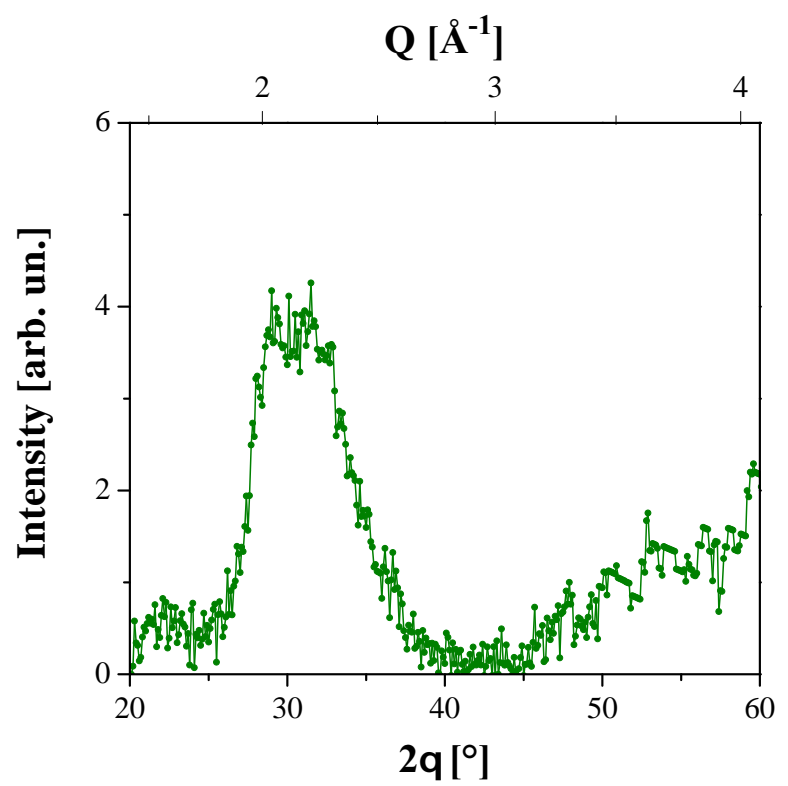

Figure 6.57. $\mathrm{X}$-ray pattern of $\mathrm{Nd}_{60} \mathrm{Fe}_{30} \mathrm{Al}_{10}$ thin film recorded with a Siemens D5000 diffractometer $\left(\mathrm{Cu}-\mathrm{K}_{\alpha}\right.$ radiation) in a Bragg-Brentano geometry. The liquid-like pattern is typical of $\mathrm{x}$-ray amorphous samples. 
However, broad diffraction peaks are expected also in the case of nanosized crystals and this fact limits the reliability of the XRD method to determine whether a material is truly amorphous or nanocrystalline. For this reason, HRTEM investigations have been
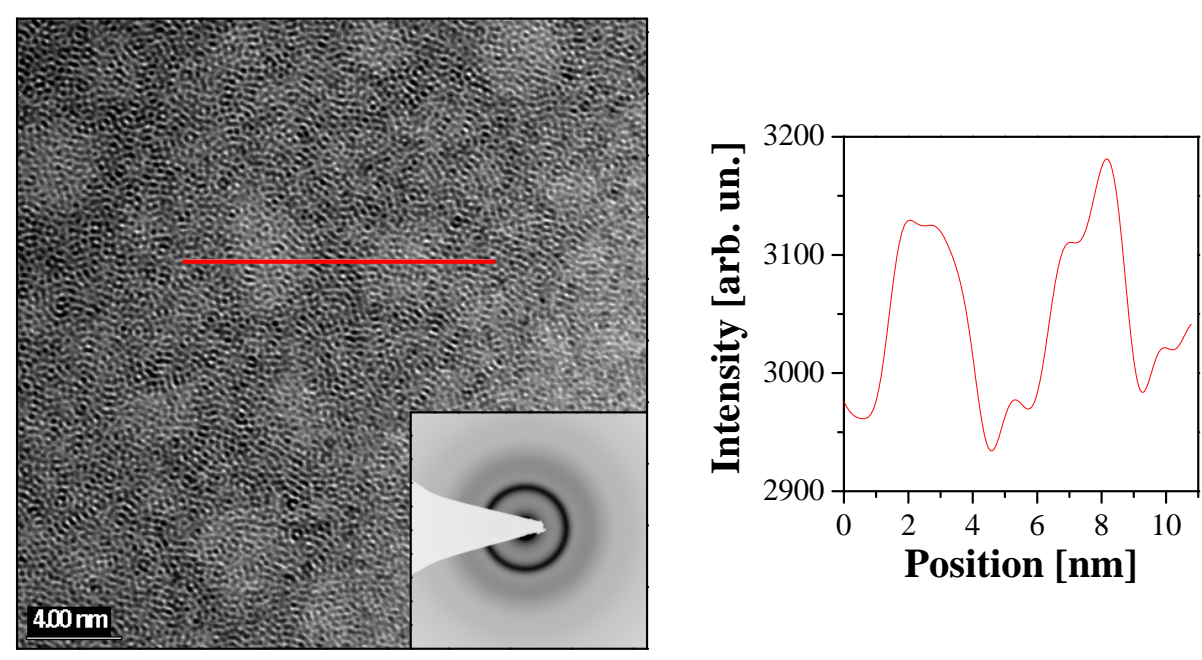

Figure 6.58. HRTEM image and $\mathrm{SAD}$ pattern of $\mathrm{Nd}_{60} \mathrm{Fe}_{30} \mathrm{Al}_{10} 50 \mathrm{~nm}$ thick film. Amorphous agglomerates (bright areas) coexist with a glassy matrix (darker areas). The mean size of the fine-dispersed phase is about $4 \mathrm{~nm}$ as evidenced from the intensity scan carried out along the line $(-)$ indicated in the image.

employed to further characterize the microstructure of the films and a representative high resolution image, together with a selected-area electron diffraction (SAD) pattern, is shown in figure 6.58. The HRTEM image has been obtained near Scherrer defocus and shows variations of the background intensity due to phase contrast indicating a complex microstructure consisting of two amorphous materials. These variations are characterized by a length scale of about $4 \mathrm{~nm}$ as evidenced from the intensity scan of the low-pass filtered version of the TEM image (cut-off at $0.75 \mathrm{~nm}^{-1}$ ) carried out along the line indicated in figure 6.58 [16]. The SAD pattern does not reveal any structural characteristics of the minor phase and shows liquid-like halos of a fully amorphous material. The TEM results confirm the tendency of the $\mathrm{Nd}_{60} \mathrm{Fe}_{30} \mathrm{Al}_{10}$ alloy to form an intrinsic composite, as previously shown for slow-cooled samples [17, 139] whose microstructure was likely to arise from a phase separation process taking place in the melt or in the undercooled liquid state. For $\mathrm{Nd}_{60} \mathrm{Fe}_{30} \mathrm{Al}_{10}$ thin films, the rapid quenching cannot completely avoid the phase separation, which results to be frozen in, but it prevents the crystallization of the formed amorphous phases. 


\subsubsection{Magnetometry}

On the basis of the structural results, magnetic characterization has been carried out in order to determine how the observed microstructure might influence the magnetic properties of the films. For this scope, several in-plane hysteresis loops have been measured

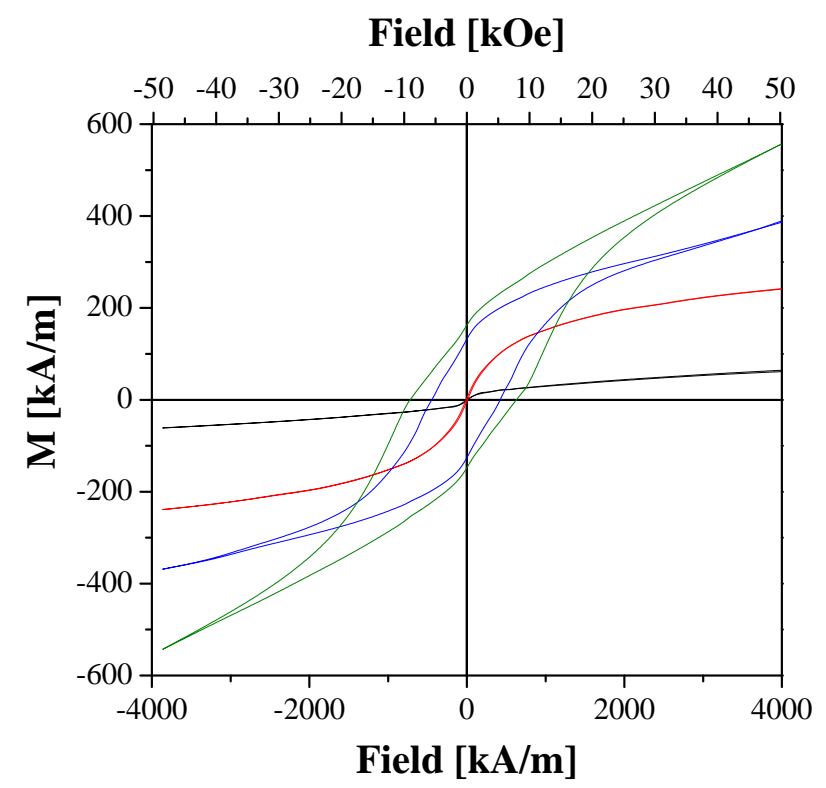

Figure 6.59. Magnetic hysteresis loops of a $50 \mathrm{~nm}$ thick $\mathrm{Nd}_{60} \mathrm{Fe}_{30} \mathrm{Al}_{10}$ film measured at $5(-), 10(-), 60(-)$ and $250 \mathrm{~K}(-)$, respectively, with the applied field parallel to the sample plane.

in the temperature range from 2 to $250 \mathrm{~K}$ and four representative curves are shown in figure 6.59. At $5 \mathrm{~K}$ the $\mathrm{Nd}_{60} \mathrm{Fe}_{30} \mathrm{Al}_{10}$ thin film shows hard magnetic properties with a coercive field $H_{c}$ of about $720 \mathrm{kA} / \mathrm{m}(\sim 9 \mathrm{kOe})$, and high values of the magnetization $M^{5 T}$ (measured at $5 \mathrm{~T}$ ) and of the remanence $M_{r}$. Moreover a closer look to the hysteresis loop reveals a typical two-phases behavior with a kink at low fields which suggests the coexistence of two magnetic phases. Upon increasing the sample temperature, $M^{5 T}, M_{r}$, and $H_{c}$ steeply decrease and as soon as $T$ approaches $40 \mathrm{~K}$, the hard magnetic properties disappear and the loops show almost no hysteretic behavior with low remanence and small coercivity which reaches the value of $9.2 \mathrm{kA} / \mathrm{m}(\sim 115 \mathrm{Oe})$ at $60 \mathrm{~K}$. The transition from the hard to the soft behavior is clearly evidenced also from the temperature dependence of the normalized magnetization $m_{5 T}(=M(T) / M(2 \mathrm{~K})$, measured at $5 \mathrm{~T})$, of the reduced remanence $m_{r}\left(=M_{r} / M_{5 \mathrm{~T}}\right)$, and of the coercivity $H_{c}$ (see figure 6.60). All the three quantities show a strong variation at $T \sim 40 \mathrm{~K}\left(T_{b}\right)$. This indicates a magnetic transition and accounts for the two-phase shape of the low-temperature hysteresis loops. Above $T_{b}$, the $\mathrm{Nd}_{60} \mathrm{Fe}_{30} \mathrm{Al}_{10}$ film indicates typical features of a low-remanence soft magnetic phase whose ordering temperature is presumably close to room temperature as deducible from the paramagnetic-like behavior at $250 \mathrm{~K}$ (see figure 6.59). These results seem to confirm 


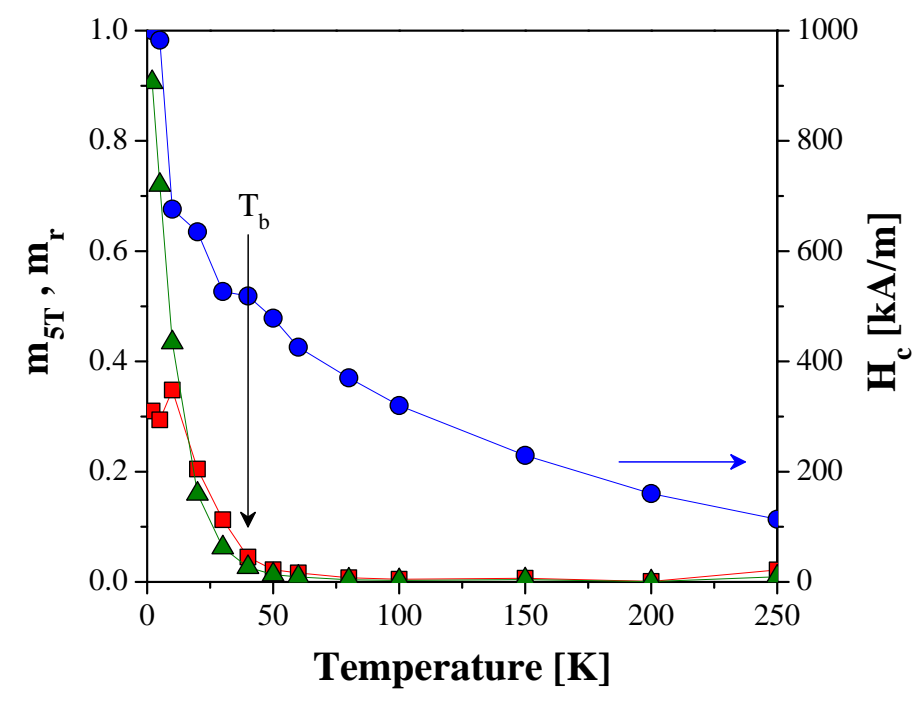

Figure 6.60. Normalized magnetization $m_{5 T}(\bullet)$, reduced remanence $m_{r}(\boldsymbol{\square})$, and coercive field $H_{c}(\boldsymbol{\Delta})$ as function of the sample temperature. The temperature $T_{b}$ at which the sample shows the transition from hard to soft magnetic behavior is indicated by the arrow.

the HRTEM investigation, which indicated the presence of two distinct phases, and show that two different temperature ranges must be distinguished to understand the magnetic behavior of the sample: $T \leq T_{b}$ and $T>T_{b}$.

In figure 6.61 we report thermomagnetic curves of zero-field cooled (ZFC) and field cooled (FC) samples, measured with an applied field of $1 \mathrm{~T}$ in the temperature range from 5 to $250 \mathrm{~K}$. As indicated by the large difference between FC and ZFC curves

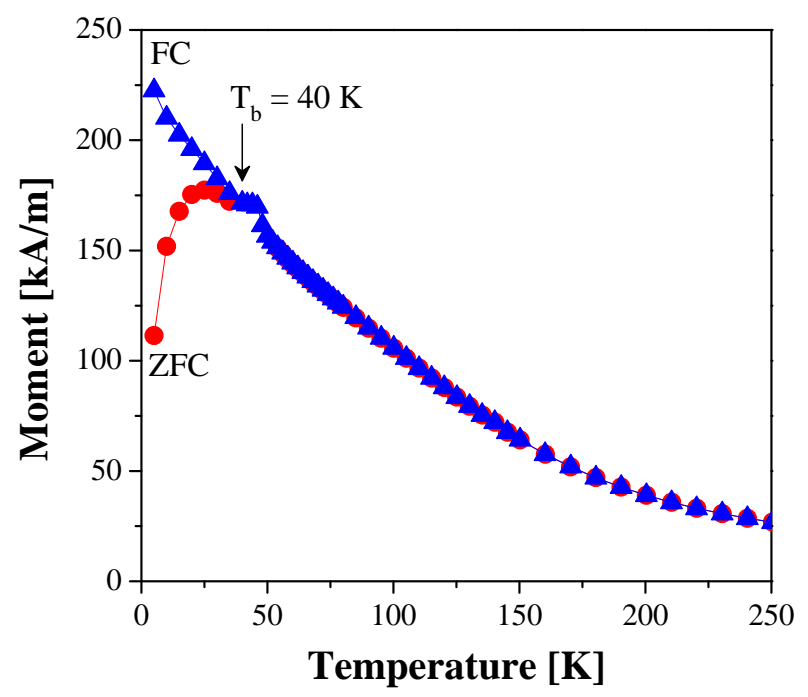

Figure 6.61. Thermomagnetic measurements taken with an applied field of $1 \mathrm{~T}$ for zerofield cooled (ZFC) and field cooled (FC) $\mathrm{Nd}_{60} \mathrm{Fe}_{30} \mathrm{Al}_{10}$ thin films from 5 to $250 \mathrm{~K}$.

at $5 \mathrm{~K}$, the magnetization depends strongly on the thermomagnetic method which is used to cool down the sample. With increasing temperature the difference decreases and 
vanishes completely at about $40 \mathrm{~K}$. This behavior has been already observed in many different rare earth-Fe systems $[17,94,151]$ and it has already been shown that they may be understood taking into account thermally activated pinning effects of the magnetic domain-walls 17, 161.

As introduced in section 4.5.1, these processes are characterized by energy barriers which must be overcome by applying an external magnetic field of a certain strength to achieve the complete unpinning. Thus, at a certain temperature and applied field, only a part of the full magnetization of the zero-field-cooled sample can be aligned, explaining the gap between FC and ZFC curves in figure 6.61.

\subsubsection{Coercivity Mechanisms}

In order to determine the characteristic aspects of pinning processes, the Gaunt formalism 63 has been taken into account to explain the temperature dependence of the coercive field $H_{c}$ observed for the thin films and to compare the results of this analysis to those of the previous slow-cooled samples.

Figure 6.62 shows the variation of $H_{c}^{1 / 2}$ as function of $T^{2 / 3}$ as deduced from the experimental data presented in figure 6.60 (right). In the low-temperature range the exper-

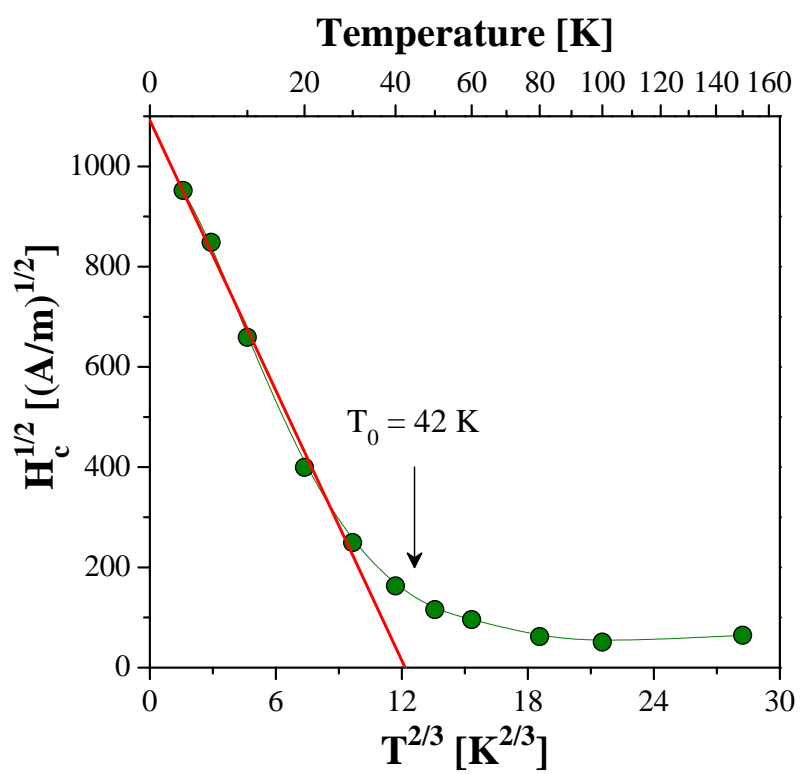

Figure 6.62. Variation of $H_{c}^{1 / 2}$ vs. $T^{2 / 3}$ for a $\mathrm{Nd}_{60} \mathrm{Fe}_{30} \mathrm{Al}_{10} 50 \mathrm{~nm}$ thick film and the relative fit $(-)$ according to the Gaunt model 63 . The characteristic crossover temperatures $T_{0}$ is indicated by the arrow.

imental curve and the Gaunt model are in good agreement as the linear fit according to equation 4.53 demonstrates. The values of the unknown parameters $H_{0}$ and $T_{0}$ are listed in table 6.17 16. 
The value of $T_{0}$ agrees with the crossover temperature $T_{b}$ which has been previously determined, suggesting that the magnetization mechanisms responsible for the shown hard magnetic behavior and for the thermomagnetic history effects are mainly governed by pinning processes. As soon as $T$ reaches $T_{0}$ the strength of the pinning centers is averaged out by thermal fluctuations and the sample shows soft magnetic behavior which depends on the intrinsic properties of the main magnetic phase.

\begin{tabular}{ccc}
\hline \multirow{2}{*}{ Parameter } & \multicolumn{3}{c}{ Value } \\
& $(1192 \pm 53) \mathrm{kA} / \mathrm{m}$ & $(4625 \pm 67) \mathrm{kA} / \mathrm{m}$ \\
\hline$H_{0}$ & $(42 \pm 4) \mathrm{K}$ & $(451 \pm 12) \mathrm{K}$ \\
$T_{0}$ & & Bulk \\
\hline
\end{tabular}

Table 6.17. Intrinsic coercive field $H_{0}$ and the crossover temperature $T_{0}$ as obtained by fitting the experimental data $H_{c}^{1 / 2}$ vs. $T^{2 / 3}$ of figure 6.62 according to the domain wall pinning model of Gaunt 63 (see equation 4.53 .

The estimation of the mean pinning sites, following the analysis proposed in previous sections (see, for example, section 6.1.4), gives out that pinning effects are strong if

$$
\frac{A^{3 / 2}}{K^{1 / 2}} \ll \frac{75 k_{B} T_{0}}{4 \pi^{4}}=5.58 \cdot 10^{-23}
$$

Therefore, assuming that the ratio between $A^{3 / 2} / K^{1 / 2}$ and $225 k_{B} T_{0} / 4 \pi^{4}$ remains equal to that used for the bulk sample (i.e., $A^{3 / 2} / K^{1 / 2}=9.3 \cdot 10^{-24} \mathrm{~J}$ ), the density of pinning sites results to be in accordance to equation 6.11

$$
\rho=8 \pi^{4} \frac{A^{3 / 2}}{K^{1 / 2}} \cdot \frac{\mu_{0} M_{s} H_{0}}{3 \cdot\left(75 k_{B} T_{0}\right)^{2}} \simeq 9.57 \cdot 10^{23} \mathrm{~m}^{-3}
$$

where $M_{s} \simeq M^{5 \mathrm{~T}}=500 \mathrm{kA} / \mathrm{m}$ (see figure 6.59). If the density is expressed as $\rho=$ $3 x /\left(4 \pi r^{3}\right)$, where $x$ is pinning sites volume fraction $(\approx 40 \%)$, the strong pinning observed for the $\mathrm{Nd}_{60} \mathrm{Fe}_{30} \mathrm{Al}_{10}$ thin films are likely due to pinning centers whose radius is approximately

$$
r=\left(\frac{3 x}{4 \pi \rho}\right)^{1 / 3} \simeq 4.6 \mathrm{~nm}
$$

The mean radius of one of the two phases visible in the TEM image of figure 6.58 is approximately a half of the value arising from the previous analysis. Nevertheless, the order of magnitude of $r$ is still in accordance with the evidences of the structural analysis and with the previous results on the slow-quenched samples.

\subsubsection{Summary}

The results on $\mathrm{Nd}_{60} \mathrm{Fe}_{30} \mathrm{Al}_{10}$ thin films confirm the tendency of this alloy to show phase separation as previously shown for splat-quenched foils and mold-cast samples of the 
same composition. However, the high cooling rate originating from the condensation method used to deposit the thin films avoid the crystallization of the two phases which result therefore to be fully amorphous. The thin films show soft magnetic properties from room temperature down to $T_{0}(=42 \mathrm{~K})$, below that temperature magnetic pinning effects control the hard magnetic behavior of the sample which shows coercive fields up to $1200 \mathrm{kA} / \mathrm{m}(\sim 15 \mathrm{kOe})$ for $T \rightarrow 0 \mathrm{~K}$ as extrapolated by the Gaunt formalism [16.

\subsection{Annealed Samples}

The results of $\mathrm{Nd}_{60} \mathrm{Fe}_{30} \mathrm{Al}_{10}$ "master alloy", bulk samples, splat-cooled foils, and thin films have evidenced a clear dependence of the alloy's properties on the cooling rate. In particular it has been shown that varying the sample preparation conditions allows to change the structural features of the specimens and to modify successfully their magnetic hardness. Moreover, all $\mathrm{Nd}_{60} \mathrm{Fe}_{30} \mathrm{Al}_{10}$ samples have been proved to be intrinsic composite materials and, hence, systems whose metastable state can be strongly influenced by ex-situ thermal treatments. The bulk samples, in particular, have been proved to undergo a coarsening process of the in-situ formed crystalline phase well below the crystallization temperature of the remanent amorphous matrix (see section 6.1.7). Due to the strong interplay between microstructure and magnetic properties (see section 6.1.8), any modification of the first is expected to influence considerably the latter.

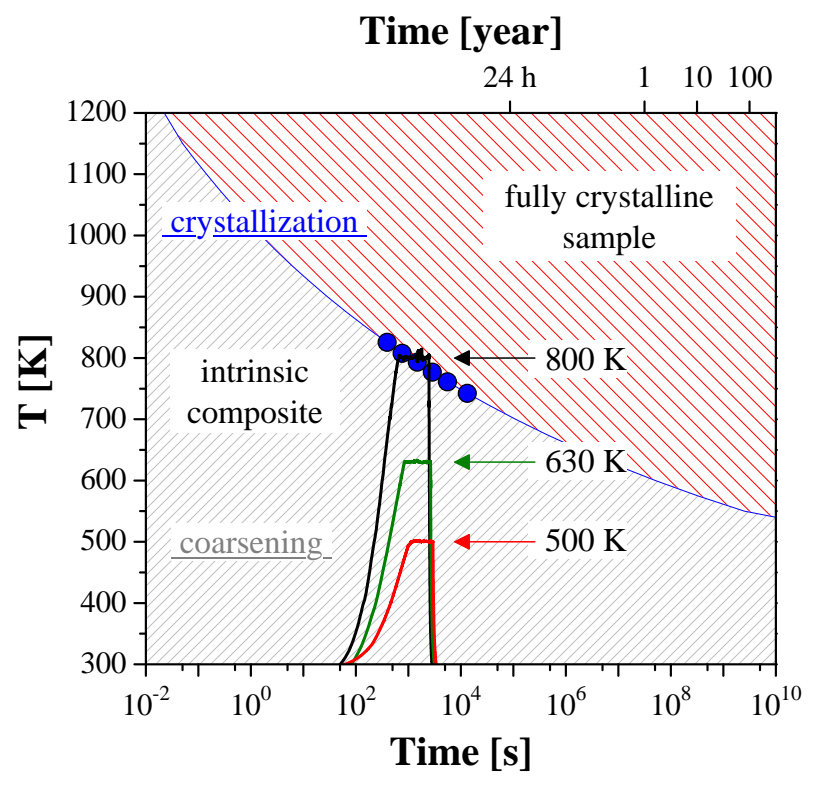

Figure 6.63. Continuous heating transformation diagram of a $\mathrm{Nd}_{60} \mathrm{Fe}_{30} \mathrm{Al}_{10}$ bulk sample $(-)$ and the time-temperature paths corresponding to annealing at $500(-), 630(-)$, and $800 \mathrm{~K}(-)$, respectively.

For this scope and on the basis of the results of section $6.1 .7 \mathrm{Nd}_{60} \mathrm{Fe}_{30} \mathrm{Al}_{10}$ bulk samples 
have been annealed far below and above the crystallization temperature of the amorphous phase by employing the experimental apparatus described in section 5.7. Structural and magnetic properties have been then investigated and interpreted on the basis of the results of the as-cast samples.

Considering the continuous heating transformation (CHT) diagram of the bulk samples obtained from the Kissinger analysis of section 6.1.7, it can be assumed that below the line which defines the crystallization event, the system is characterized, in good approximation, only by a coarsening process (see figure 6.63) while, above it, the full transformation of the amorphous phase occurs. Thus, three different bulk samples have been annealed for 30 min respectively at 500,630, and $800 \mathrm{~K}$ following the time-temperature paths shown in figure 6.63. The evolution of the magnetic properties upon annealing is presented in the following two sections.

\subsubsection{Annealing at 500 and $630 \mathrm{~K}$}

Figure 6.64 shows the temperature dependence of the magnetic moment in a field $\mu_{0} H=$ $5 \mathrm{~T}$ recorded for the as-cast sample (sample A) and for the specimens annealed at 500 (sample B) and $630 \mathrm{~K}$ (sample C) for $30 \mathrm{~min}$. No difference emerges from the ther-

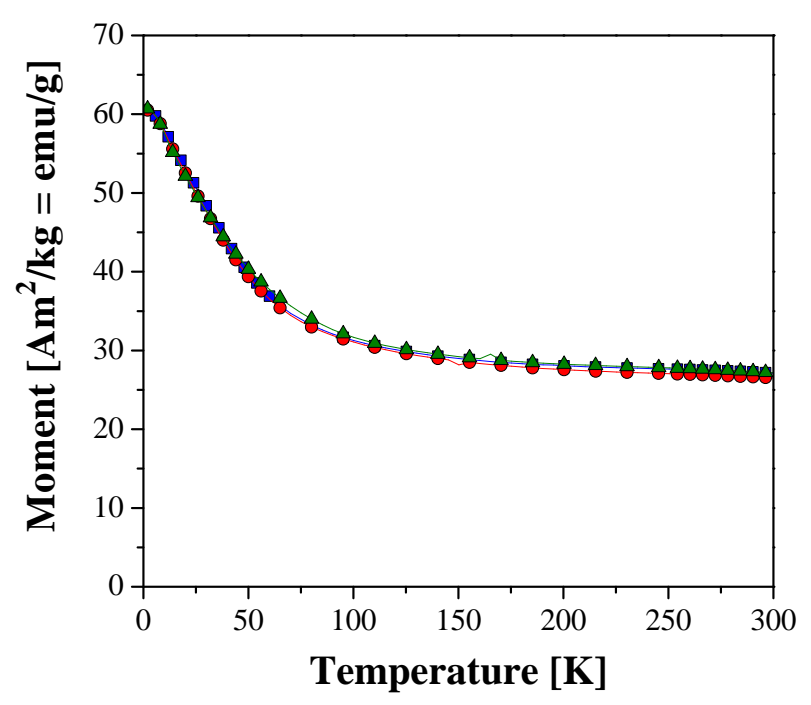

Figure 6.64. Thermomagnetic curves at $5 \mathrm{~T}$ of an as-cast bulk sample ( $\boldsymbol{\square})$ and for the two specimens annealed at $500(\mathrm{O})$ and $630 \mathrm{~K}(\boldsymbol{\Delta})$, respectively.

momagnetic curves of the three samples indicating that their magnetic behavior is not significantly affected by the annealing. However, the temperature dependence of the coercive field in the same temperature range (see figure 6.65) shows that the annealed samples are magnetically softer at $300 \mathrm{~K}$ than the as-cast ones and become progressively harder as the temperature decreases. This behavior can also be shown by plotting $H_{c}$ vs. $T$ within the Gaunt formalism. 

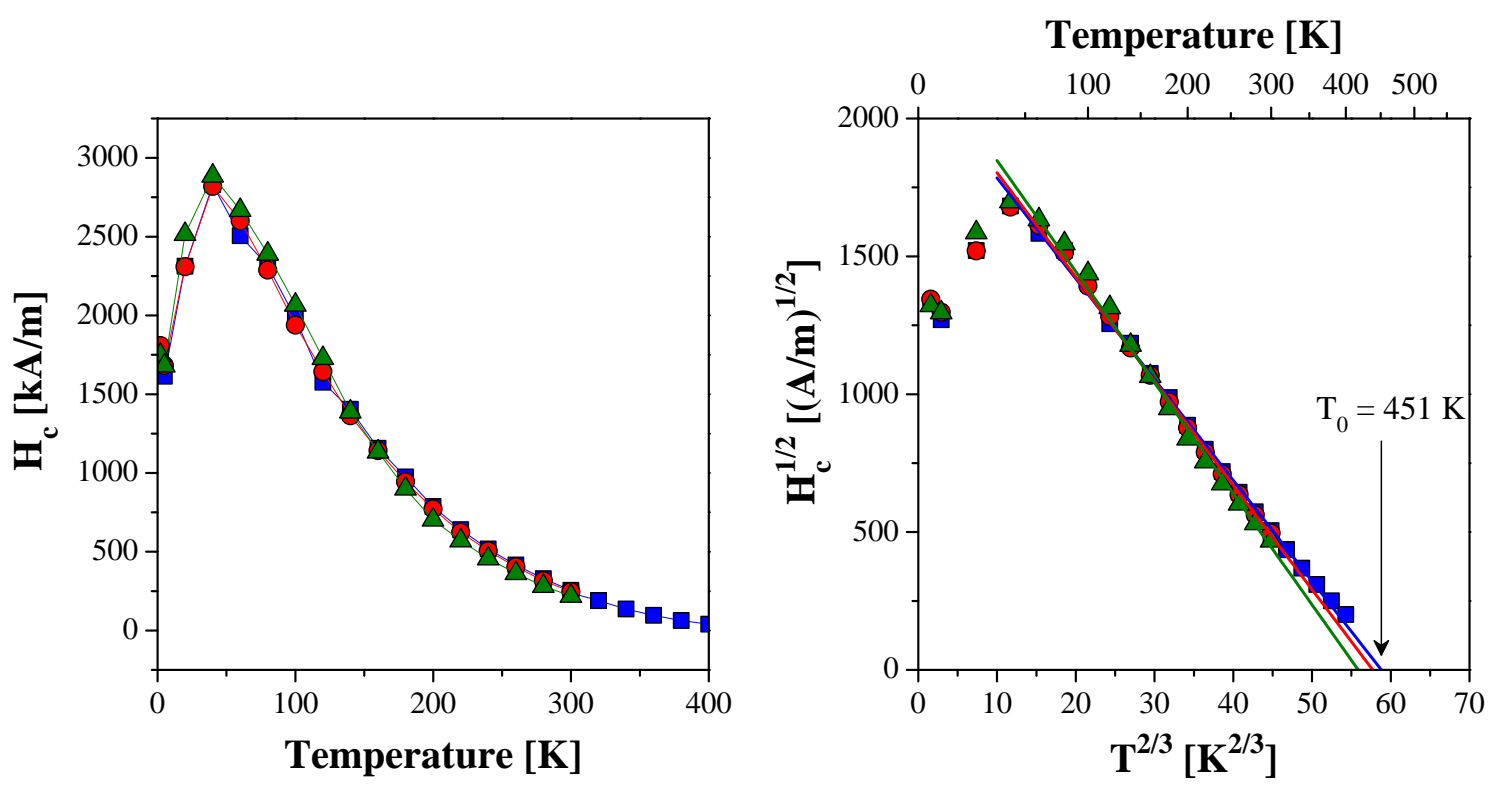

Figure 6.65. (Right) Temperature dependence of the coercivity field of as-cast and annealed $\mathrm{Nd}_{60} \mathrm{Fe}_{30} \mathrm{Al}_{10}$ bulk samples (A: $\square$; B: O; C: $\boldsymbol{\Delta}$ ) in the temperature range from 2 to $300 \mathrm{~K}$. (Left) Gaunt's plot $H_{c}^{1 / 2}$ vs. $T^{2 / 3}$ relative to the experimental data shown on the left graph. The lines are the linear fit between 40 and $300 \mathrm{~K}$ according to equation 4.53 .

The curves $H_{c}^{1 / 2}$ vs. $T^{2 / 3}$ are in good accordance with the Gaunt model in the temperature range from 40 to about $300 \mathrm{~K}$ and show significant differences of the slopes and of the intercepts. The linear regressions of the experimental data allow to determine the unknown parameters $H_{0}$ and $T_{0}$ of equation 4.53 . Their values are reported in table 6.18 .

\begin{tabular}{cccc}
\hline \multirow{2}{*}{ Parameter } & Sample A & Sample B & Sample C \\
\hline$H_{0}$ & $(4625 \pm 67) \mathrm{kA} / \mathrm{m}$ & $(4758 \pm 86) \mathrm{kA} / \mathrm{m}$ & $(5404 \pm 138) \mathrm{kA} / \mathrm{m}$ \\
$T_{0}$ & $(451 \pm 12) \mathrm{K}$ & $(438 \pm 17) \mathrm{K}$ & $(407 \pm 21) \mathrm{K}$ \\
\hline
\end{tabular}

Table 6.18. Intrinsic coercive field $H_{0}$ and the crossover temperature $T_{0}$ as obtained by fitting the experimental data $H_{c}^{1 / 2}$ vs. $T^{2 / 3}$ of figure 6.65 (right) according to the domain wall pinning model of Gaunt 63 . (see equation 4.53).

These results suggest that the tailoring of the hard magnetic properties of the $\mathrm{Nd}_{60} \mathrm{Fe}_{30} \mathrm{Al}_{10}$ bulk sample is effectively possible by annealing in the time-temperature region where the system undergoes the coarsening process. Small variations of the microstructure influence therefore the pinning mechanisms which determine the coercivity of this sample and the hard magnetic behavior can be consequently modified. 


\subsubsection{Annealing at $800 \mathrm{~K}$}

The investigation of the magnetic properties of the bulk samples annealed above the crystallization temperature of the amorphous phase may be helpful to understand the lowtemperature magnetic behavior of the as-prepared samples (i.e., bulk and splat-cooled foils). In fact, the magnetic contribution of the crystalline phase ordering at $\sim 50 \mathrm{~K}$ arises more clearly since the crystallized sample is paramagnetic at room temperature as it can be deduced from the magnetization $M(H)$ recorded at $300 \mathrm{~K}$ (see figure 6.66).

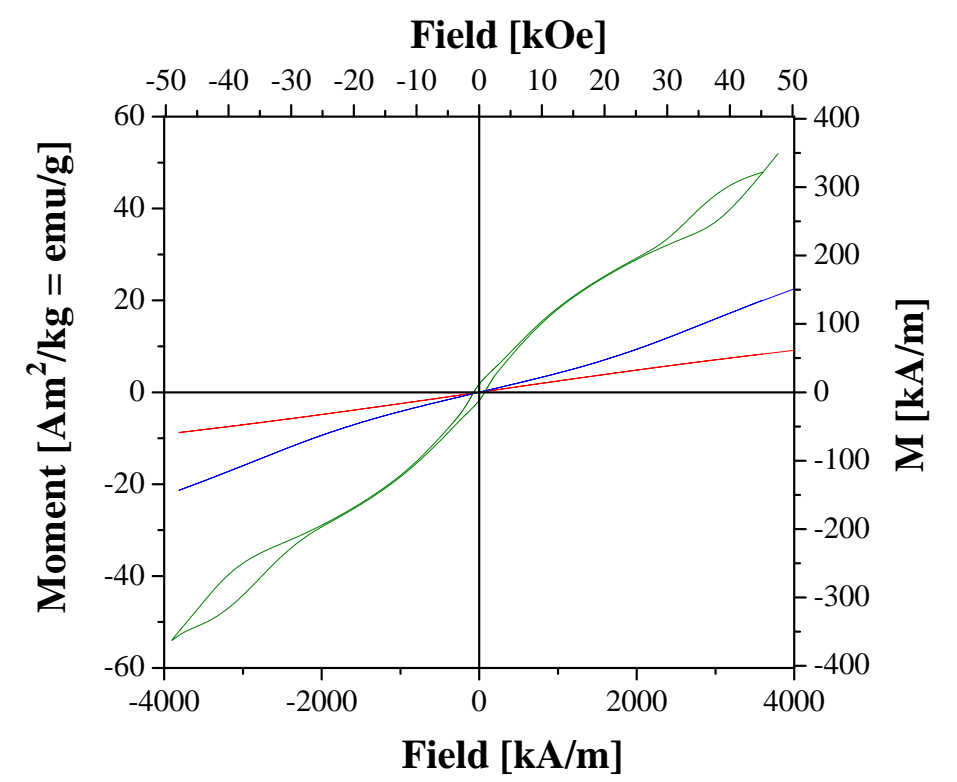

Figure 6.66. Hysteresis loops of a $\mathrm{Nd}_{60} \mathrm{Fe}_{30} \mathrm{Al}_{10}$ bulk sample annealed at $800 \mathrm{~K}$ for $30 \mathrm{~min}$ recorded at $5(-), 140(-)$, and $300 \mathrm{~K}(-)$, respectively.

Below about $250 \mathrm{~K}$, the influence of a second phase starts to affect $M(H)$ which shows a strong change of the curve slope between low and high applied field (see, for example, the curve recorded at $140 \mathrm{~K}$ in figure 6.66). This behavior has been attributed to an antiferromagnetic phase with Néel temperature at about $260 \mathrm{~K}\left(\mathrm{Nd}_{6} \mathrm{Fe}_{11-x} \mathrm{Al}_{3+x}\right.$ [46, 180, 181|). As the temperature reaches $80 \mathrm{~K}$ a soft magnetic phase appears and $M(H)$ shows an additional narrow hysteresis loop at low fields. Triyono has identified this phase as the soft $\mathrm{NdAl}_{2}$ which orders ferromagnetically at about $75 \mathrm{~K}$ [158. At very low temperatures, when the original crystalline $\mathrm{Nd}$-rich phase becomes magnetically ordered, the magnetization curve shows an additional hysteresis loop at high field. This behavior can be ascribed to the ferromagnetic ordering of the $\mathrm{Nd}$-rich nanocrystals and to a complex metamagnetic transition observed in the $\mathrm{Nd}_{6} \mathrm{Fe}_{11-x} \mathrm{Al}_{3+x}$-like phase [50, 87, 118.

These results have been confirmed by AC magnetometry measurements. The temperature dependence of the real $\left(\chi^{\prime}\right)$ and of the imaginary $\left(\chi^{\prime \prime}\right)$ AC susceptibility measured between 4 and $300 \mathrm{~K}$ is shown in Figure 6.67. The $\chi^{\prime}(T)$ curve presents clearly three peaks at $75\left(T_{1}\right), 28\left(T_{2}\right)$, and $8 \mathrm{~K}\left(T_{3}\right)$, respectively. 


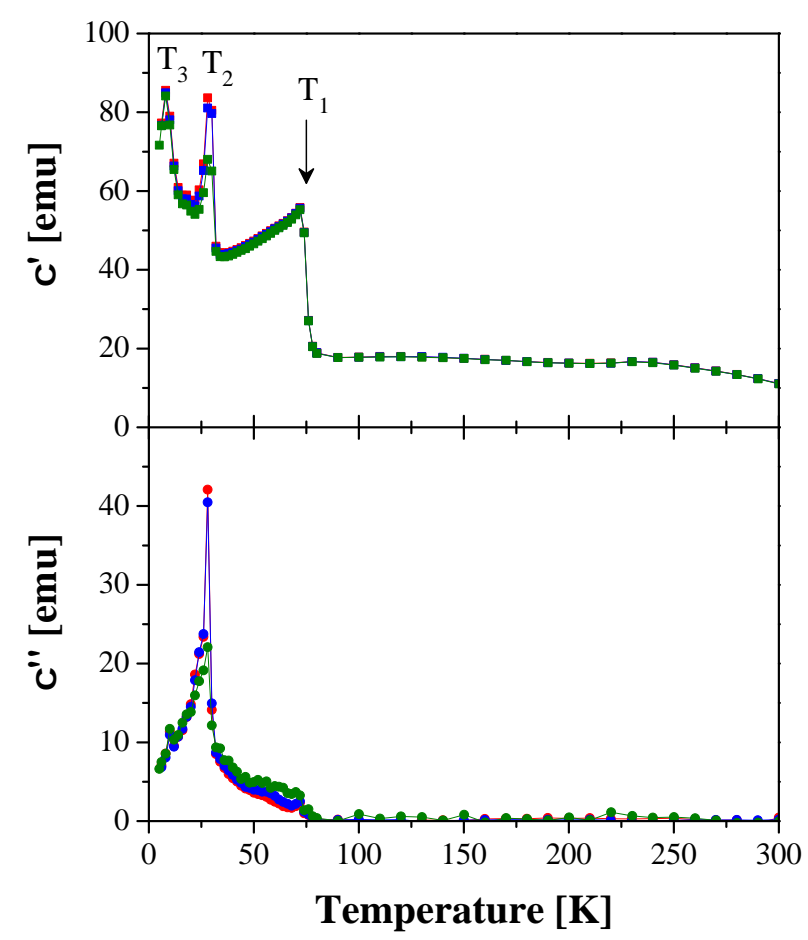

Figure 6.67. Real (top) and imaginary (bottom) AC susceptibility of a fully crystalline bulk sample recorded with a SQUID magnetometer in the temperature range from 4 to $300 \mathrm{~K}$. The different curves have been measured with a frequency of $1(-), 10(-)$, and $117 \mathrm{~Hz}(-)$, (driving field: $4 \mathrm{Oe})$.

The appearance of the transitions at $T_{3}$ and $T_{2}$ confirms the results obtained on the as-cast sample and the hypothesis that they may be related to the ordering temperatures of metallic Nd (at 8.2 and $19.9 \mathrm{~K}$, respectively [84, p. 57]). Furthermore, it is worth to notice the dependence of the peak at $T=28 \mathrm{~K}$ from the driving frequency of the external AC field (see figure 6.67). This behavior is commonly observed in spin-glass magnetic systems or frustrated ferromagnets [58,59], where many metastable spin configurations coexist separated by a distribution of energy barriers, and it may be an indication of magnetic moments at inequivalent sites which couple ferromagnetically in the crystalline dhcp phase. This magnetic structure may be responsible for the strong increase of the magnetization and the variation of the hysteresis loop shape observed in the as-cast and annealed samples below $40 \mathrm{~K}$, and for the singular hysteresis curves shown in figure 6.66. 



\section{Chapter 7}

\section{Concluding Remarks}

Aim of this work has been the structural and magnetic characterization of the system $\mathrm{Nd}_{60} \mathrm{Fe}_{30} \mathrm{Al}_{10}$. This alloy has attracted much attention during the last few years as a potential candidate for magnetic applications and due to the high glass-forming ability [80]. Its magnetic properties have been found to be sensitively dependent on the cooling rate with which the samples are prepared [139,152], and that has inspired the systematic study carried out in this thesis about the origin of the alloy's high coercive field and the cooling rate-dependent properties.

This chapter summarizes the results and the preeminent findings achieved by the characterization of four different types of $\mathrm{Nd}_{60} \mathrm{Fe}_{30} \mathrm{Al}_{10}$ samples prepared by cooling rates ranging from some $\mathrm{K} / \mathrm{s}$ up to $10^{10} \mathrm{~K} / \mathrm{s}$ and proposes possible investigations which may contribute to further understand their properties.

\subsection{Summary and Conclusions}

This thesis has shown that the use of different preparation techniques makes it possible to fabricate $\mathrm{Nd}_{60} \mathrm{Fe}_{30} \mathrm{Al}_{10}$ samples with cooling rates varying by several orders of magnitude. Structural and magnetic properties of "master alloy" specimens, bulk samples, splatcooled foils, and thin films have proven the tendency of the $\mathrm{Nd}_{60} \mathrm{Fe}_{30} \mathrm{Al}_{10}$ alloy to form an intrinsic composite consisting of at least two phases which are likely to arise from a phase separation process taking place in the melt or in the undercooled liquid. Master alloy, bulk samples, and splat foils cooled with a rate lower than about $10^{6} \mathrm{~K} / \mathrm{s}$ are composed of a Nd-rich crystalline phase embedded in a remanent amorphous matrix. The volume fraction and the size of the crystalline grains decreases as the cooling rate increases. The thin films (cooling rate of about $10^{10} \mathrm{~K} / \mathrm{s}$ ) on the other hand show a fully amorphous microstructure consisting of two amorphous phases which have been successfully observed by TEM.

Phase separation is a direct consequence of chemical and thermodynamical properties 
of an alloy (differences of the heats of mixing, local minima in the composition dependence of the Gibbs free energy, atomic species diffusivity, etc.), and the cooling rate determines the path through phase separation and crystallization which further minimizes the Gibbs free energy. For $\mathrm{Nd}_{60} \mathrm{Fe}_{30} \mathrm{Al}_{10}$ thin films, the rapid quenching cannot completely avoid the phase separation but it prevents the crystallization of the intrinsically formed amorphous phases. The crystallization of the Nd-rich phase results instead to be favored in the slow-cooled $\mathrm{Nd}_{60} \mathrm{Fe}_{30} \mathrm{Al}_{10}$ samples.

As anticipated in chapter 2, glass formation (i.e., glass forming ability) is enhanced in alloys which are multicomponent systems with more than three elements, whose chemical composition is closer to the eutectic point, and whose constituents have a large negative heat of mixing $\Delta H_{m}$ leading to low interdiffusion constants. $\mathrm{Nd}_{60} \mathrm{Fe}_{30} \mathrm{Al}_{10}$ is a multicomponent system and its composition is close to the eutectic points of the $\mathrm{Nd}-\mathrm{Fe}$ and $\mathrm{Nd}-\mathrm{Al}$ phase diagrams (see figure 6.5), however, the different heat of mixing between the three elements and the competitive driving forces which arise from them may be responsible for the observed phase separation. This result seems to confirm the tendency of others multicomponent eutectic glass-forming alloys to separate in two or more phases upon rapid quenching from the melt or the undercooled liquid state 22, 138.

The structural characterization carried out by high-energy wide angle $\mathrm{x}$-ray scattering, small-angle neutron scattering, transmission electron microscopy and differential scanning calorimetry has proven that the samples are intrinsic composites.

For the bulk specimens and splat foils, in particular, the in-situ formed crystalline phase has revealed an unexpected mass fractal distribution $M(r) \propto\left(r / r_{0}\right)^{d_{f}}$ with a fractal dimension $d_{f}$ that decreases as the cooling rate increases. The morphology and the density of a mass fractal are not constant but vary with $r$, where $r$ is the lateral dimension measured from the center of the fractal cluster. As $r$ increases the density of the fractal nucleus decreases while the volume fraction of the Fe-rich matrix progressionally increases inducing a gradient in Nd and Fe concentrations. The embedding amorphous matrix is ferromagnetic with a high saturation field. This observation agrees with the results of several other rare earth-transition metal systems for which a non-collinear sperimagnetic structure has been hypothesized and applied fields up to $30 \mathrm{~T}$ are necessary to reach the saturation state. By the use of polarized neutron scattering, it has been proven that mass fractal clusters are several nanometers in size and consist of a paramagnetic nucleus and of a magnetic shell whose thickness is few nanometers. The mass fractals form a network over the whole sample and its complex microstructure together with the local variation of the magnetic properties at the interface with the amorphous phase act as pinning centers for the ferromagnetic matrix. To explain the structural, chemical, and magnetic characteristics of the pinning sites, a core-shell model has been developed (see section 6.1.8). Pinning behavior has been evidenced by magnetization curves $M(H)$ as well as by the temperature dependence of the coercive field $H_{c}$. According to the Gaunt 
formalism [63], inhomogeneous systems characterized by strong pinning (see section 4.5.1) present a variation of the coercivity as a function of temperature expressed by the equation $\left(H_{c} / H_{0}\right)^{1 / 2}=1-\left(T / T_{0}\right)^{2 / 3}$. At $T=0 \mathrm{~K}$ the system shows an intrinsic coercivity $H_{0}$ which is determined by the density and the force of the pinning centers. Above $T_{0}$ thermal fluctuations overcome the energy barriers of the pinning process (i.e., the strength of the pinning sites is averaged out) and the coercivity mechanisms of the samples are then governed by magnetization reversal taking place in the amorphous matrix and, hence, by its effective anisotropy.

The pinning behavior $H_{c}^{1 / 2}$ vs. $T^{2 / 3}$ has been observed in all the $\mathrm{Nd}_{60} \mathrm{Fe}_{30} \mathrm{Al}_{10}$ specimens and the values of the parameters $H_{0}$ and $T_{0}$ have shown a strong dependence on the cooling rate indicating an effective interplay between coercivity mechanisms and microstructure (for instance, $T_{0}$ decreases from about $450 \mathrm{~K}$ for the bulk samples to $290 \mathrm{~K}$ for the splat-cooled foils and to approximately $40 \mathrm{~K}$ for the thin films). Furthermore, in the rapid-quenched splats for which the Curie temperature is significantly higher than the activation temperature $T_{0}$, it has been possible to show the transition at $T_{0}$ from the pinning-type behavior to a nucleation-type magnetization reversal proving the reliability of the Gaunt formalism in these magnetic systems.

This result indicates that it is possible to control the magnetic properties (e.g., coercive field) of the $\mathrm{Nd}_{60} \mathrm{Fe}_{30} \mathrm{Al}_{10}$ alloy by changing the preparation method of the samples. However, since intrinsic composites are metastable materials, variations of the specimen coercivity may also be induced by post-preparation thermal treatments. This issue has been addressed in particular for the bulk samples which have shown the highest values of the coercive field and of the Gaunt temperature $T_{0}$. The thermal stability has been determined by applying the Kissinger analysis to DSC scans recorded at different heating rates and by in-situ high-temperature WAXS. The results have demonstrated that the Nd-rich crystalline phase undergoes a coarsening process well below the crystallization temperature of the amorphous matrix. The calorimetric signal arising from the grain coarsening masks the glass transition explaining why the $\mathrm{Nd}_{60} \mathrm{Fe}_{30} \mathrm{Al}_{10}$ alloy has not shown a supercooled liquid region in traditional differential scanning calorimetry measurements. Furthermore, the results on the $\mathrm{Nd}_{60} \mathrm{Fe}_{30} \mathrm{Al}_{10}$ alloy have shown the relevance of the combination of diffraction and differential scanning calorimetry in the study of intrinsic composites and suggest a possible wider use of this method to a larger class of materials undergoing complex structural evolution. The coarsening offers the possibility to influence the complex morphology of the crystalline phase to which the pinning effects have been ascribed. Samples annealed in the temperature range where coarsening occurs have shown that the Gaunt formalism can still be applied to the temperature dependence of the coercivity but the values of the parameters $H_{0}$ and $T_{0}$ change slightly as a consequence of changed pinning conditions.

In conclusion, this work has proven that the magnetic features of $\mathrm{Nd}_{60} \mathrm{Fe}_{30} \mathrm{Al}_{10}$ com- 
posites strongly depend on the structural properties of the samples. The tailoring of the hard magnetic characteristics is therefore possible either by changing the cooling rate through the use of different preparation methods (or the preparation conditions) or by ex-situ annealing. Although the $\mathrm{Nd}_{60} \mathrm{Fe}_{30} \mathrm{Al}_{10}$ alloy has been defined as a candidate for possible technological applications, the modest value of the product $\left(\mu_{0} M \cdot H\right)_{\max }$, the relatively low Curie temperature, and its reduced thermal stability may be serious obstacles for the realization of commercial products based on this intrinsic composite. Nevertheless, the many interesting properties and the complex structural and magnetic behavior makes the $\mathrm{Nd}_{60} \mathrm{Fe}_{30} \mathrm{Al}_{10}$ alloy a candidate for future scientific investigations.

\subsection{Outlook}

Many questions about the structural and magnetic properties of the $\mathrm{Nd}_{60} \mathrm{Fe}_{30} \mathrm{Al}_{10}$ intrinsic composites have not yet been addressed by this work nor by other research groups. For example: Is it possible to prove directly the pinning effects of the crystalline phase on the domain walls of the ferromagnetic matrix? How do the intrinsic properties of the Nd-rich nanocrystals influence the low-temperature magnetic behavior of the alloy? May other magnetic phases be present and responsible for the observed temperature variation of the magnetic properties?

The answers to these questions presuppose the possibility to use some more specific investigation techniques than those employed for this work. Many valuable alternatives exist and some unique possibilities are offered, for example, by

- $\mathrm{x}$-ray magnetic circular dichroism (XMCD): XMCD is a powerful technique in the investigation of a magnetic system due to the element and electron-shell sensitivity of the spectroscopic method. The possibility to separate the specific contributions of $\mathrm{Nd}$ and Fe may be a unique opportunity to understand the magnetic properties of this alloy.

- polarized small-angle neutron scattering (pSANS): the use of pSANS during this work has been limited to room temperature and a maximal applied field $\mu_{0} H=$ 1.2 T. The possibility to use ancillary equipments (e.g., cryostat, high-field magnet, etc.) may be useful to verify the room temperature results and determine the effective role of the low $-T_{C}$ phase.

- neutron diffraction: the use of neutron diffraction in the determination of the magnetic structure of a crystalline alloy is well established. Therefore, on the basis of the results observed for the annealed bulk sample, it would be of great interest to measure the $\mathrm{Nd}_{60} \mathrm{Fe}_{30} \mathrm{Al}_{10}$ composite by neutron diffraction and at low temperature. 
- magnetic force microscopy (MFM): the direct observation of the domain structure of the ferromagnetic Fe-rich matrix may be of great help to confirm the hypothesis of domain wall pinning effects. MFM offers the opportunity to perform temperatureand magnetic field-dependent measurements without the necessity of particular sample preparation (compared, for example, to Lorentz transmission electron microscopy) which is of fundamental importance to avoid their oxidation.

The $\mathrm{Nd}_{60} \mathrm{Fe}_{30} \mathrm{Al}_{10}$ alloy may therefore well be a system of future interest. 



\section{Appendix A}

\section{Fitting Processes}

The data analysis and the fitting curves presented in this work were mostly done using the Advanced Fitting Tool (AFT) of the software package OriginPro@ (OriginLab Corporation). This fitting tool is based on the so-called Levenberg-Marquardt method which is a popular alternative to the Gauss-Newton method of finding the minimum of any function $\chi^{2}(x)$ that is a sum of squares of nonlinear functions

$$
\chi^{2}(\boldsymbol{p})=\frac{1}{m-l} \sum_{i=1}^{m} w_{i} \cdot\left[y_{i}-f(\boldsymbol{x}, \boldsymbol{p})\right]^{2}
$$

where $\boldsymbol{y}=\left(y_{1}, \ldots, y_{m}\right)$ is an observation, $\boldsymbol{w}=\left(w_{1}, \ldots, w_{m}\right)$ is a vector of constants ("weights"), $f(x)$ is the function which described the theoretical model believed to explain the process that produced the experimental data $\boldsymbol{y}$, and $\boldsymbol{x}=\left(x_{1}, \ldots, x_{n}\right)$ is a vector of independent variables. The model usually depends on one or more parameters $\boldsymbol{p}=\left(p_{1}, \ldots, p_{l}\right)$. The aim of the fitting procedure is to find the vector $\hat{\boldsymbol{p}}$ which best describe the data (best fit). The standard way of defining the best fit is to choose the parameters so that the sum of the squares of deviations of the theoretical curve from the experimental points is at its minimum. Thus, the AFT tries to find a minimum of $\chi^{2}(\boldsymbol{p})$ on a $(l+1)$-dimensional surface defined as $S\left(\chi^{2}(\boldsymbol{p}), \boldsymbol{p}\right)$ with a finite number of iterations. The results of the fitting process is hence that vector $\hat{\boldsymbol{p}}$ for which $\chi^{2}(\hat{\boldsymbol{p}})$ is minimized and, hence, $f(\boldsymbol{x}, \hat{\boldsymbol{p}})$ is closest to $y$.

The components of the vector $\boldsymbol{w}$ represent the relative weight of the $m$ experimental points $\boldsymbol{y}$ in the minimization process. Two different weighting methods were employed:

$$
w_{i}=1
$$

(no weight) and

$$
w_{i}=\frac{1}{\left(\Delta y_{i}\right)^{2}}
$$

(instrumental weight), where $\Delta y_{i}$ are the experimental errors. 
The minimization algorithm minimizes $\chi^{2}(\boldsymbol{p})$ by initializing the values of the parameters and then by performing a series of iterations which involve the computation at each stage of $\chi^{2}$ and of its derivatives $d \chi^{2} / d \boldsymbol{p}$. For this reason and due to the complexity of some of the functions $f(\boldsymbol{x}, \boldsymbol{p})$, all the fitting expressions have been written in $\mathrm{C}++$ and compiled in a Dynamic Link Library (DLL). The AFT can access in this way the DLL at any iteration of the minimization process instead of interpreting the function written in the internal Origin language and the fitting section results to be speeded up by a factor hundred.

The quality of the fitting can be deduced from the values of $\chi^{2}$ itself and/or of the coefficient of determination $R^{2}(\in[0,1])[154$ which are automatically calculated by the $\mathrm{AFT}$ at the end of the minimization process. 


\section{Appendix B}

\section{SANS Form Factors}

As previously seen in section 5.3.2, the form factor $F(\boldsymbol{Q}, R)$ is a function that describes how the cross section $d \sigma / d \Omega(Q)$ is modulated by interference effects between radiation scattered by different parts of the same scattering body. Consequently it is very dependent on the shape and on the size $R$ of the scattering body. The general form of $F(\boldsymbol{Q}, R)$ is given by Van de Hulst's equation 164

$$
|F(\boldsymbol{Q}, R)|^{2}=\frac{1}{V_{p}^{2}(R)}\left|\int_{V_{p}(R)} \exp [i f(\boldsymbol{Q} \cdot \boldsymbol{r})] d \boldsymbol{r}\right|^{2}
$$

where $V_{p}$ is the volume of the scattering body, $R$ is a "shape parameter" and $f$ is a function of $Q$ and $R$.

Several analytic expressions of $|F(\boldsymbol{Q}, R)|^{2}$ for most common shapes has been calculated starting from the equation 5.36. The most important are: the form factor for a sphere of radius $R$

$$
|F(\boldsymbol{Q}, R)|^{2}=\left|\frac{3[\sin (Q R)-Q R \cos (Q R)]}{(Q R)^{3}}\right|^{2}
$$

for a disc of negligible thickness and radius $R$

$$
|F(\boldsymbol{Q}, R)|^{2}=\frac{2}{(Q R)^{2}} \cdot\left[1-\frac{J_{1}(2 Q R)}{Q R}\right]
$$

( $J_{1}$ is a first-order Bessel function), and the Debye form factor for exponential-decaying correlation with crossover-length $R[47$

$$
|F(\boldsymbol{Q}, R)|^{2}=|F(0)|^{2} \cdot\left[\frac{1}{1+(Q R)^{2}}\right]
$$

Experimentally, however, it is of greater interest the case of a set of spheres with a distribution of radii. Equation 5.36 can consequently be modified to take into account the function which expresses the shape of the distribution, however an analytical expression for the form factor is not easily obtainable. Considering for example a log-normal 
distribution, the form factor would be

$$
|F(\boldsymbol{Q}, R)|^{2}=|F(0)|^{2} \cdot\left[\frac{1}{1+(Q R)^{2}}\right]
$$

In those cases, the value of $|F(\boldsymbol{Q}, R)|^{2}$ can be obtained by calculating numerically the integral in equation $\mathrm{B} .5$ for each value of $Q$. 


\section{Appendix C}

\section{Mass Fractals}

With the term morphology are commonly indicated all those features which characterize the microstructure of a material: geometry, topology, and surface structure. The geometry describes the shapes and sizes of the micro- and mesoscale elements of the material. The shapes can vary from completely ordered patterns (in general, if the material has been formed under close-to-equilibrium conditions) to complex and chaotic ones. The topology describes how the elements are connected to one another and the surface describes how they interact with the surrounding environment (especially for those materials made under far-from-equilibrium conditions). Examples of disordered and complex morphologies are numerous and one of the best known is the fractal [35, 132, 148]. "In a fractal structure, the part is reminiscent of the whole" 135 . The system is self-similar and possesses scaleinvariant properties (i.e., its morphology repeats itself at different length scales): there exist pieces of the system above a certain length scale (lower cutoff length for fractality) that can be magnified to recover the structure of the system at larger length scales up to another length scale (the upper cutoff length). Below the lower cutoff and above the upper cutoff scales the system loses its self-similarity.

The importance of the concept of mass fractal in the study of $\mathrm{Nd}_{60} \mathrm{Fe}_{30} \mathrm{Al}_{10}$ alloy emerges clearly from the following consideration. If a fractal morphology is to be formed at small length scale, it will require formation of a large surface that separates the material from the embedding space. Since formation of a large surface requires a large amount of energy, fractal materials do not usually form under equilibrium conditions. Hence, a rapid-quenched composite becomes an ideal candidate for the possible observation of fractal behavior in natural systems. Natural systems are however generally not fractal, in the strict mathematical sense [112, 114], nevertheless, their behavior approaches the fractal geometry and the use of fractals is important for describing them.

One of the simplest characteristics of a fractal is its fractal dimension $d_{f}$. If we cover the fractal system by non-overlapping $d$-dimensional spheres of Euclidean radius $r$ and count the number $N(r)$ of such spheres that is required for complete coverage, the fractal 
dimension of the system is then defined by (box-counting method) 165

$$
d_{f}=\lim _{r \rightarrow 0} \frac{\ln N}{\ln (1 / r)}
$$

Non-fractal objects (i.e., a straight line, a square or a sphere) are Euclidean and their effective dimensionality is $d$. In order to be able to write down equation C.1, it has implicitly been assumed the existence of a lower and an upper cutoff length scale for the fractality of the system which are, respectively, the radius $r_{0}$ of the spheres and the linear size $L$ of the system. One may also define the fractal dimension $d_{f}$ can also be defined through the relation between the system's mass $M$ and its lateral dimension $r$. If the system is composed of particles of radius $r_{0}$ and mass $m$, then

$$
M=c \cdot m\left(\frac{r}{r_{0}}\right)^{d_{f}}
$$

where $c$ is a geometrical constant $(\simeq 1)$ and $2 \leq d_{f} \leq 3$. Equation C.1 implies that the mean density $\rho=M / \Omega$, where $\Omega$ is the volume of the system that contains the fractal in the embedding space, is not a constant but decreases with the length scale $r$ according to the relation

$$
\rho \sim r^{d_{f}-d} \quad\left(\sim M^{\frac{\left(d_{f}-d\right)}{d_{f}}}\right)
$$

A fractal dimension less than the Euclidean dimension of the space and a decreasing density with increasing length scales both imply that the fractal object cannot fill the space and has a sparse structure (see figure C.1).

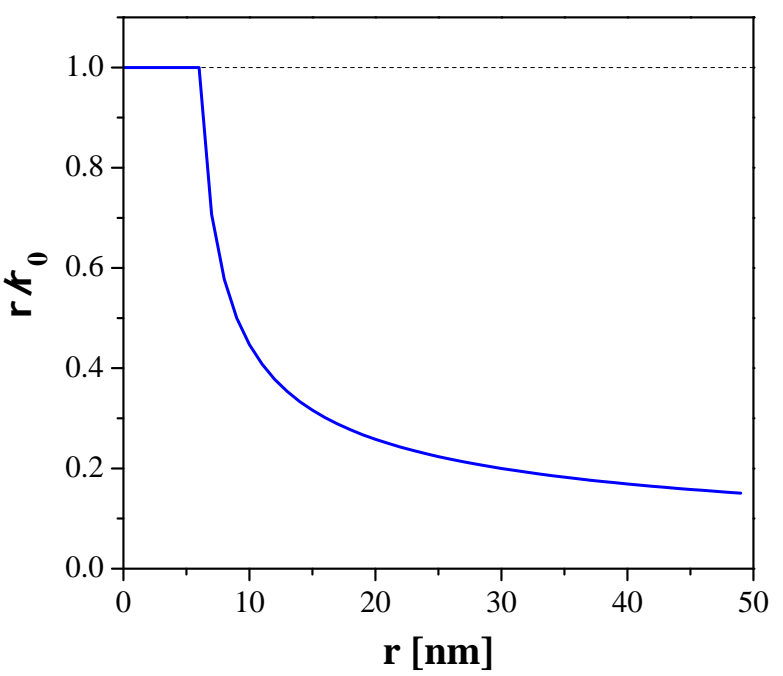

Figure C.1. Density $\rho(r)$ of a fractal object with a lower cutoff length of $5 \mathrm{~nm}$ and fractal dimension $d_{f}=2.5$. $\rho$ has been normalized to the value $\rho_{0}$ of a non-fractal object.

One of the most important examples of such fractals is that generated by the diffusionlimited aggregation model [176] (see figure C.2). Extensive computer simulations indicate that $d_{f} \simeq 2.5$ for $3-\mathrm{D}$ aggregates 122 . 


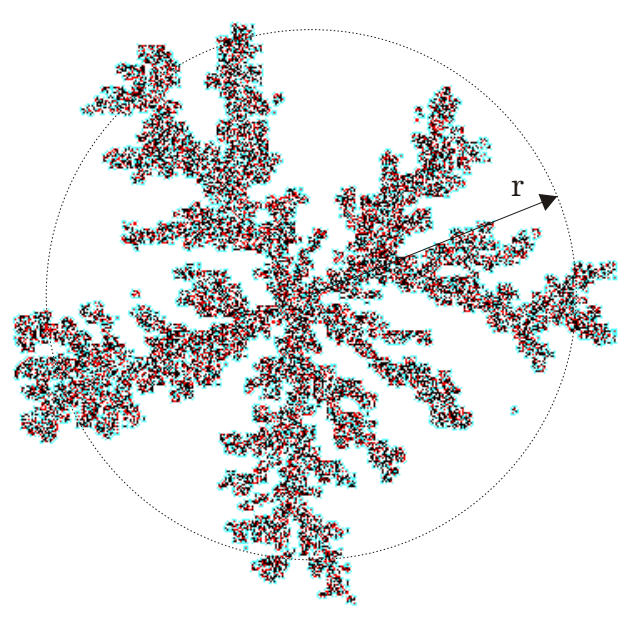

Figure C.2. A two-dimensional diffusion-limited aggregate.

A different method to determine the fractal dimension is the use of small angle scattering (SAS) 8, 113, 115, 137. In a SAS experiment the observed scattering cross section is proportional to the Fourier transform of the direct correlation function $C(r)$ which describes the material elements distribution 165

$$
\frac{d \sigma}{d \Omega} \propto \int_{0}^{+\infty} C(\boldsymbol{r}) \exp (i \boldsymbol{Q} \cdot \boldsymbol{r}) d \boldsymbol{r}
$$

For an isotropic medium, $C(\boldsymbol{r})=C(r)$ and equation C.4 becomes

$$
\frac{d \sigma}{d \Omega} \propto \int_{0}^{+\infty} C(r) 4 \pi r^{2} \frac{\sin (Q r)}{Q r} d r
$$

Since the correlation function for a $3-\mathrm{D}$ system is given by $C(r) \sim r^{d_{f}-3} 62,135$

$$
\frac{d \sigma}{d \Omega} \sim Q^{-d_{f}} \cdot \Gamma\left(d_{f}-1\right) \sin \left[\frac{\pi}{2}\left(d_{f}-1\right)\right]
$$

where $\Gamma$ is the gamma function. Equation C.6 is valid if $\left(Q \cdot R_{p}\right) \ll 1$ and $\left(Q \cdot R_{m}\right) \gg 1$, where $R_{p}$ and $R_{m}$ are, respectively, the effective radii of an individual scattering particle and the material.

As discussed above, the range of scale-invariance and fractal behavior in real materials may be limited by the lower and upper cutoff length scales $r_{0}$ and $L$. scattering. To remedy this situation, Sinha 62 included in the expression of $C(r)$ an exponentially decaying term, incorporating a scattering correlation length $\xi_{s}$, which reflects the upper limit $L$ of self-similarity. Due to this term

$$
C(r) \sim r^{d_{f}-3} \cdot \exp \left(-\frac{r}{\xi_{s}}\right)
$$


which yields 62,155

$$
\frac{d \sigma}{d \Omega} \sim \xi_{s}^{d_{f}} \cdot \frac{\Gamma\left(d_{f}-1\right)}{Q \xi_{s}} \cdot \frac{\sin \left[\left(d_{f}-1\right) \arctan \left(Q \xi_{s}\right)\right]}{\left[1+\left(Q \xi_{s}\right)^{2}\right]^{\frac{\left(d_{f}-1\right)}{2}}}
$$

Note that in the limit $\xi_{s} \rightarrow+\infty$, the previous relation becomes equation C.6, and for small $Q \xi_{s}$ and $d_{f}=3$ (i.e., homogeneous material)

$$
\frac{d \sigma}{d \Omega} \sim \frac{1}{1+\left(Q \xi_{s}\right)^{2}}
$$

which is the classical Debye relation [47] (see appendix B and equation B.4).

When the surface is rough and the roughness pattern is self-similar, the surface itself is fractal and it may be characterized by a fractal dimension $d_{s}\left(2 \leq d_{s} \leq 3\right)$. The surface fractal dimension $d_{s}$, may or may not be the same as the fractal dimension $d_{f}$ of the bulk. This implies that a non-fractal material may have a fractal surface, and vice-versa.

If both the bulk material and the surface are fractal and $d_{f} \neq d_{s}$, the cross section in a SAS experiment becomes proportional to

$$
\frac{d \sigma}{d \Omega} \sim Q^{d_{s}-2 d_{f}}
$$

which reduces to $Q^{-4}$ (the classical Porod law, see equation B.2 for non-fractal objects with smooth surfaces $\left(d_{f}=3, d_{s}=2\right)$, or to $Q^{d_{s}-6}$ for a non-fractal objects with fractal surfaces $\left(d_{f}=3\right)$, or finally to $Q^{-d_{f}}$ for a so-called mass fractal for which $d_{f} \equiv d_{s}$.

The general expression of the cross-section can then be expressed by the relation

$$
\frac{d \sigma}{d \Omega}=B+P \cdot F^{2}\left(Q, r_{0}\right) \cdot \xi_{s}^{d_{f}} \cdot \frac{\Gamma\left(d_{f}-1\right)}{Q \xi_{s}} \cdot \frac{\sin \left[\left(d_{f}-1\right) \arctan \left(Q \xi_{s}\right)\right]}{\left[1+\left(Q \xi_{s}\right)^{2}\right]^{\frac{\left(d_{f}-1\right)}{2}}}
$$

where $B$ and $P$ are two constants, and $F^{2}\left(Q, r_{0}\right)$ is the form factor for a sphere of radius $r_{0}$ (see equation B.2) which accounts for the non-fractal behavior for $r<r_{0}$.

In order to characterize the mass fractal behavior of the $\mathrm{Nd}_{60} \mathrm{Fe}_{30} \mathrm{Al}_{10}$ alloy, small angle neutron scattering has been employed for the determination of the mass fractal dimension $d_{f}$ and of the upper cutoff length $\xi_{s}$. 


\section{Bibliography}

[1] A. Aharoni, Introduction to the theory of ferromagnetism, Clarendon Press, Oxford, 1996.

[2] R. Alben, J. J. Becker, and M. C. Chi, "Random anisotropy in amorphous ferromagnets", J. Appl. Phys. 49, 1653 (1978).

[3] J. Almer, U. Lienert, R. L. Peng, C. Schlauer, and M. Odén, "Strain and texture analysis of coatings using high-energy x-rays", J. Appl. Phys. 94, 697 (2003).

[4] C. A. Angell, K. L. Ngai, G. B. McKenna, P. F. McMillan, and S. W. Martin, "Relaxation in glass-forming liquids and amorphous solids", J. Appl. Phys. 88, 3113 (2000).

[5] J. Arcas, A. Hernando, J. M. Barandiarán, M. Vázquez C. Prados, P. Marín, and A. Neuweiler, "Soft to hard magnetic anisotropy in nanostructured magnets", Phys. Rev. B 58, 5193 (1998).

[6] A. Arrott and J. E. Noakes, "Approximate equation of state for Nickel near its critical temperature", Phys. Rev. Lett. 19, 786 (1967).

[7] N. W. Ashcroft and N. D. Mermin, Solid State Physics, Saunders College Publishing, Fort Worth, 1976.

[8] G. Beaucage, "Small-angle scattering from polymeric mass fractals of arbitrary mass-fractal dimension", J. Appl. Crystallogr. 29, 134 (1996).

[9] H. Beck and H.-J. Güntherodt, "Introduction to Metallic Glasses", In H.-J. Güntherodt and H. Beck, editors, Glassy Metals, Vol. 1, pages 1-18. Springer-Verlag, Berlin, 1981.

[10] E. Belorizky, M. A. Fremy, J. P. Gavigan, D. Givord, and H. S. Li, "Evidence in rare-earth ( $\mathrm{R}$ )-transition metal (M) intermetallics for a systematic dependence of R-M exchange interactions on the nature of the R atom", J. Appl. Phys. 61, 3971 (1987).

[11] J. D. Bernal, "Co-ordination of randomly packed spheres", Nature 188, 410 (1960).

[12] G. Bertotti, Hysteresis in Magnetism, Academic Press, San Diego, 1998. 
[13] S. Blundell, Magnetism in Condensed Matter, Oxford University Press, Oxford, 2001.

[14] A. Bracchi, L. Del Bianco, and E. Bonetti, (unpublished).

[15] A. Bracchi, K. Samwer, J. Almer, R. Aravinda Narayanan, P. Thiyagarajan, and S. Schneider, "Separation of grain growth and glass transition during structural evolution of the bulk $\mathrm{Nd}_{60} \mathrm{Fe}_{30} \mathrm{Al}_{10}$ alloy", Phys. Rev. B 70, 172105 (2004).

[16] A. Bracchi, K. Samwer, T. Niermann, M. Seibt, and S. Schneider, "Phase separation and magnetic properties of $\mathrm{Nd}_{60} \mathrm{Fe}_{30} \mathrm{Al}_{10}$ ", Appl. Phys. Lett. 85, 2565 (2004).

[17] A. Bracchi, K. Samwer, S. Schneider, and J. F. Löffler, "Random anisotropy and domain-wall pinning process in the magnetic properties of rapidly quenched $\mathrm{Nd}_{60} \mathrm{Fe}_{30} \mathrm{Al}_{10} "$, Appl. Phys. Lett. 82, 721 (2003).

[18] A. Bracchi, K. Samwer, S. Schneider, P. Schaaf, and J. F. Löffler, "Phase separation and microstructure controlled magnetic properties of rapidly quenched $\mathrm{Nd}_{60} \mathrm{Fe}_{30} \mathrm{Al}_{10} "$, Mater. Sci. Eng. A 375-377, 104 (2004).

[19] A. Bracchi, S. Schneider, P. Thiyagarajan, and K. Samwer, "Cooling rate dependent microstructure and magnetic properties of the glass forming alloy $\mathrm{Nd}_{60} \mathrm{Fe}_{30} \mathrm{Al}_{10}$ ", J. Magn. Magn. Mater. 272-276, 1423 (2004).

[20] U. Buchenau, "Neutronenstreuung I", In 30. IFF Ferienkurs, Vol. 1, page 13.1. Jülich, 1999.

[21] W. Buckel and R. Hilsch, "Einfluss der kondensation bei tiefen temperaturen auf den elektrischen widerstand und die supraleitung fur verschiedene metalle", $Z$. Phys. 138, 109 (1954).

[22] R. Busch, S. Schneider, A. Peker, and W. L. Johnson, "Decomposition and primary crystallization in undercooled $\mathrm{Zr}_{41.2} \mathrm{Ti}_{13.8} \mathrm{Cu}_{12.5} \mathrm{Ni}_{10.0} \mathrm{Be}_{22.5}$ melts", Appl. Phys. Lett. 67, 1544 (1995).

[23] K. H. J. Buschow, "Intermetallic compounds of Rare-Earth and 3d transition metals", Rep. Prog. Phys. 40, 1179 (1977).

[24] K. H. J. Buschow, "Magnetic properties of amorphous Rare-Earth-Cobalt alloys", J. Appl. Phys. 51, 2795 (1980).

[25] K. H. J. Buschow, "Amorphous Alloys", In K. A. Gschneidner and L. Erying, editors, Handbook on the physics and chemistry of rare earths, page 52. Elsevier, Amsterdam, 1983.

[26] K. H. J. Buschow and A. M. van der Kraan, "Magnetic properties of amorphous Rare-Earth-Iron alloys", J. Magn. Magn. Mater. 22, 220 (1981).

[27] R. W. Cahn, "Metallic glasses", Contemp. Phys. 21, 43 (1980). 
[28] P. Caldirola, R. Cirelli, and G. M. Prosperi, Introduzione alla fisica teorica, UTET, Torino, 1993.

[29] E. Callen, Y. J. Liu, and J. R. Cullen, "Initial magnetization, remanence, and coercivity of the random anisotropy amorphous ferromagnet", Phys. Rev. B 16, 263 (1977).

[30] G. S. Cargill, "Structural investigation of noncrystalline Nickel-Phosphorus alloys", J. Appl. Phys. 41, 12 (1970).

[31] H. S. Chen, "Glassy metals", Rep. Prog. Phys. 43, 353 (1980).

[32] H. S. Chen and M. Goldstein, "Anomalous viscoelastic behavior of metallic glasses of Pd-Si-based alloys", J. Appl. Phys. 43, 1642 (1972).

[33] H. S. Chen and D. Turnbull, "Thermal evidence of a glass transition in Gold-SiliconGermanium alloy", Appl. Phys. Lett. 10, 284 (1967).

[34] L. C. Chen and F. Spaepen, "Analysis of calorimetric measurements of grain growth", J. Appl. Phys. 69, 679 (1991).

[35] S. H. Chen and J. Teixeira, "Structure and fractal dimension of protein-detergent complexes", Phys. Rev. Lett. 57, 2583 (1986).

[36] C. L. Chien and K. M. Unruh, "Magnetic properties and hyperfine interactions in concentrated Fe-Ag alloys", Phys. Rev. B 28, 1214 (1983).

[37] S. Chikazumi, Physics of magnetism, John Wiley \& Sons, New York, 1986.

[38] H. Choi-Yim, R. Busch, and W. L. Johnson, "The effect of silicon on the glass forming ability of the $\mathrm{Cu}_{47} \mathrm{Ti}_{34} \mathrm{Zr}_{11} \mathrm{Ni}_{8}$ bulk metallic glass forming alloy during processing of composites", J. Appl. Phys. 83, 7993 (1998).

[39] H. Choi-Yim, J. Schroers, and W. L. Johnson, "Microstructures and mechanical properties of tungsten wire/particle reinforced $\mathrm{Zr}_{57} \mathrm{Nb}_{5} \mathrm{Al}_{10} \mathrm{Cu}_{15.4} \mathrm{Ni}_{12.6}$ metallic glass matrix composites", Appl. Phys. Lett. 80, 1906 (2002).

[40] T. W. Clyne and P. J. Withers, An introduction to metal matrix composites, Cambridge University Press, Cambridge, 1995.

[41] R. W. Cochrane, R. Harris, and M. J. Zuckermann, "The role of structure in the magnetic properties of amorphous alloys", Phys. Rep. 48, 1 (1978).

[42] J. M. D. Coey, "Amorphous Magnetic Order", J. Appl. Phys. 49, 1646 (1978).

[43] J. J. Croat, "Magnetic hardening of $\mathrm{Pr}-\mathrm{Fe}$ and Nd-Fe alloys by melt-spinning", $J$. Appl. Phys. 53, 3161 (1982).

[44] B. D. Cullity, Elements of x-ray diffraction, Addison-Wesley, London, 1978.

[45] H. A. Davies, J. Aucote, and J. B. Hull, "The kinetics of formation and stabilities of metallic glasses", Scripta Metall. 8, 1179 (1974). 
[46] C. H. de Groot, K. H. J. Buschow, and F. R. de Boer, "Magnetic properties of $\mathrm{R}_{6} \mathrm{Fe}_{13-x} \mathrm{M}_{1+x}$ compounds and their hydrides", Phys. Rev. B 57, 11472 (1998).

[47] P. Debye, H. R. Anderson, and H. Brumberger, "Scattering by an inhomogeneous solid. II. The correlation function and its application", J. Appl. Phys. 28, 679 (1957).

[48] N. M. Dempsey, M. Ghidini, J. P. Nozières, P. A. I. Smith, B. Gervais, and J. M. D. Coey, "Magnetic hardening of $\mathrm{Sm}_{2} \mathrm{Fe}_{17} \mathrm{~N}_{3}$ by radiation damage", Phys. Rev. Lett. 81, 5652 (1998).

[49] J. Ding, Y. Li, and X. Z. Wang, "The coercivity of rapidly quenched $\mathrm{Nd}_{60} \mathrm{Fe}_{30} \mathrm{Al}_{10}$ alloys", J. Phys. D 67, 1544 (1995).

[50] J. Ding, L. Si, Y. Li, and X. Z. Wang, "Magnetoresistivity and metamagnetism of the $\mathrm{Nd}_{33} \mathrm{Fe}_{50} \mathrm{Al}_{17}$ alloy", Appl. Phys. Lett. 75, 1763 (1999).

[51] P. Duwez, "Structure and properties of alloys rapidly quenched from liquid state", Trans. Am. Soc. Metals 60, 607 (1967).

[52] P. Duwez, "Metallic Glasses - Hystorical Background", In H.-J. Güntherodt and H. Beck, editors, Glassy Metals, Vol. 1, pages 19-23. Springer-Verlag, Berlin, 1981.

[53] T. Egami, "Magnetic amorphous alloys: physics and technological applications", Rep. Prog. Phys. 47, 1601 (1984).

[54] T. Egami, P. J. Flanders, and C. D. Graham, "Amorphous alloys as soft magnetic materials", AIP Conf. Proc. 24, 697 (1975).

[55] S. R. Elliott, Physics of amorphous materials, Longman Scientific \& Technical, Harlow, 1990.

[56] W. Felsch, "Ferromagnetism of amorphous iron", Z. Phys. 219, 280 (1969).

[57] W. Felsch, "Ferromagnetism of amorphous gold-iron alloy thin films", Z. Angew. Phys. 29, 217 (1970).

[58] K. H. Fischer and J. A. Hertz, Spin Glasses, Cambridge University Press, Cambridge, 1993.

[59] M. E. Fisher, "The theory of equilibrium critical phenomena", Rep. Prog. Phys. 30, 615 (1967).

[60] J. R. Frade, C. M. Queiroz, and M. H. Fernandes, "Simulated effects of transient nucleation on the crystallization of glass samples", J. Non-Cryst. Solids 333, 263 (2004).

[61] F. C. Frank, "Supercooling of liquids", Proc. R. Soc. London A 43, 215 (1952). 
[62] T. Freltoft, J. K. Kjerms, and S. K. Sinha, "Power-law correlations and finite-size effects in silica particles aggregates studied by small-angle neutron scattering", Phys. Rev. B 33, 269 (1986).

[63] P. Gaunt, "Ferromagnetic domain wall pinning by a random array of inhogeneities", Philos. Mag. B 48, 261 (1983).

[64] P. Gaunt and G. J. Roy, "Magnetic viscosity in ferromagnets. II. Co 5 Ce permanent magnet particles", Philos. Mag. 34, 781 (1976).

[65] B. C. Giessen, In T. Masumoto and K. Suzuki, editors, 4th Int. Conf: on Rapidly Quenched Metals, Vol. 1, page 213, Sendai, 1982. Japan. Inst. Metals.

[66] A. L. Greer, "Metallic glasses", Science 267, 1947 (1995).

[67] A. I. Gubanov, "Quasi-classical theory of amorphous ferromagnetics", ).

[68] P. Haasen, Physical Metallurgy, Cambridge University Press, Cambridge, 1996.

[69] G. C. Hadjipanayis and A. Kim, "Domain wall pinning versus nucleation of reversed domains in R-Fe-B magnets", J. Appl. Phys. 63, 3310 (1988).

[70] G. C. Hadjipanayis, W. Tang, Y. Zhang, S. T. Chui, J. F. Liu, C. Chen, and H. Kronmüller, "High temperature 2:17 magnets: relationship of magnetic properties to microstructure and processing", IEEE T. Magn. 36, 3382 (2000).

[71] 0. Halpern and M. H. Johnson, "On the magnetic scattering of neutrons", Phys. Rev. 55, 898 (1939).

[72] R. Harris, M. Plishke, and M. J. Zuckermann, "New model for amorphous magnetism", Phys. Rev. Lett. 31, 160 (1973).

[73] C. C. Hays, C. P. Kim, and W. L. Johnson, "Microstructure controlled shear band pattern formation and enhanced plasticity of bulk metallic glasses containing in situ formed ductile phase dendrite dispersions", Phys. Rev. Lett. 84, 2901 (2000).

[74] N. Heiman, K. Lee, I. Potter, and S. Kirkpatrick, "Modified mean-field model for Rare-Earth-Iron amorphous alloys", J. Appl. Phys. 47, 2634 (1976).

[75] D. W. Henderson, "Thermal analysis of non-isothermal crystallization kinetics in glass forming liquids", J. Non-Cryst. Solids 30, 301 (1979).

[76] R. Hilsch and W. Martienssen, "Gitterstörung in dünnen Schichten", Nuovo Cim. 7 (2), 480 (1958).

[77] R. P. Hjelm, "The resolution of TOF low-Q diffractometers: instrumental, data acquisition and reduction factors", J. Appl. Phys. 21, 618 (1988).

[78] A. Inoue, A. Takeuchi, and T. Zhang, "Bulk Nd-Fe-Al amorphous alloys with hard magnetic properties", Met. Mater. Tran. A 29, 1779 (1998). 
[79] A. Inoue, T. Zhang, and A. Takeuchi, "Hard magnetic bulk amorphous alloys", IEEE T. Magn. 33, 3814 (1997).

[80] A. Inoue, T. Zhang, W. Zhang, and A. Takeuchi, "Bulk Nd-Fe-Al amorphous alloys with hard magnetic properties", Mater. Trans., JIM 37, 99 (1996).

[81] A. Inoue, T. Zhang, W. Zhang, and A. Takeuchi, "Hard magnetic bulk amorphous $\mathrm{Nd}-\mathrm{Fe}-\mathrm{Al}$ alloys of $12 \mathrm{~mm}$ in diameter made by suction casting", Mater. Trans., JIM 37, 636 (1996).

[82] J. F. Janak, "Uniform susceptibilities of metallic elements", Phys. Rev. B 16, 255 (1977).

[83] J. Jensen, "Magnetic structures and excitations in rare-earth metals", In M. Akhavan, J. Jensen, and K. Kitazawa, editors, Magnetic and Superconducting Materials, Vol. B, pages 711-726. World Scientific, Singapore, 2000.

[84] J. Jensen and A. R. Mackintosh, Rare Earth Magnetism, Clarendon Press, Oxford, 1998.

[85] D. Jiles, Introduction to magnetism and magnetic materials, Chapman \& Hall, London, 1998.

[86] W. L. Johnson, "Bulk metallic glasses-A new engineering material", Curr. Opin. Sol. State Phys. 1, 383 (1996).

[87] S. Jonen, H. R. Rechenberg, and J. Campo, "Rare earth effects on the magnetic behavior of $\mathrm{R}_{6} \mathrm{Fe}_{11-x} \mathrm{Al}_{3+x}$ compounds", J. Magn. Magn. Mater. 242-245, 803 (2002).

[88] K. F. Kelton, G. W. Lee, A. K. Gangopadhyay, R. W. Hyers, T. J. Rathz, J. R. Rogers, M. B. Robinson, and D. S. Robinson, "First x-ray scattering studies on electrostatically levitated metallic liquids: Demonstrated influence of local icosahedral order on the nucleation barrier", Phys. Rev. Lett. 90, 195504 (2003).

[89] M. Kersten, Phys. Z. 44, 63 (1943).

[90] D. Kim, B. L. Zink, F. Hellman, and J. M. D. Coey, "Critical behavior of La ${ }_{0.75} \mathrm{Sr}_{0.25} \mathrm{MnO}_{3} "$, Phys. Rev. B 65, 214424 (2002).

[91] H. E. Kissinger, "Variation of the peak temperature with heating rate in Differential Thermal Analysis", J. Research Natl. Bur. Standards 57, 217 (1956).

[92] H. E. Kissinger, "Reaction kinetics in Differential Thermal Analysis", Anal. Chem. 29, 1702 (1957).

[93] W. Klement, R. H. Willens, and P. Duwez, "Non-crystalline structure in solidified Gold-Silicon alloys", Nature 187, 869 (1960). 
[94] H. Z. Kong, J. Ding, Z. L. Dong, L. Wang, T. White, and Y. Li, "Observation of clusters in $\mathrm{RE}_{60} \mathrm{Fe}_{30} \mathrm{Al}_{10}$ alloys and the associated magnetic properties", J. Phys. D 35, 423 (2002).

[95] K. Koyama, H. Fujii, and P. C. Canfield, "Magnetocrystalline anisotropy of a $\mathrm{Nd}_{2} \mathrm{Fe}_{17}$ single crystal", Phys. B 226, 363 (1996).

[96] J. Kramer, Z. Phys. 106, 675 (1937).

[97] M. J. Kramer, A. S. O’Connor, K. W. Dennis, R. W. McCallum, L. H. Lewis, L. D. Tung, and N. P. Duong, "Origins of coercivity in the amorphous alloy Nd-Fe-Al", IEEE T. Magn. 37, 2497 (2001).

[98] H. Kronmüller, K.-D. Durst, and M. Sagawa, "Analysis of the magnetic hardening mechanism in RE-FeB permanent magnets", Phys. Z. 74, 291 (1988).

[99] G. Kumar, J. Eckert, W. Löser, S. Roth, and L. Schultz, "Effect of Al on microstructure and magnetic properties of mould-cast $\mathrm{Nd}_{60} \mathrm{Fe}_{40-x} \mathrm{Al}_{x}$ alloys", Scripta Mater. 48, 321 (2003).

[100] G. Kumar, J. Eckert, S. Roth, W. Löser, L. Schultz, and S. Ram, "Effect of microstructure on the magnetic properties of mold-cast and melt-spun $\mathrm{Nd}-\mathrm{Fe}-$ Co-Al amorphous alloys", Acta Mater. 51, 229 (2003).

[101] G. Kumar, J. Eckert, S. Roth, K. H. Müller, and L. Schultz, "Coercivity mechanism in mold-cast $\mathrm{Nd}_{60} \mathrm{Fe}_{x} \mathrm{Co}_{30-x} \mathrm{Al}_{10}$ bulk amorphous alloys", J. Alloy Compd. 348, 309 (2003).

[102] F. J. G. Landgraf, F. P. Missell, H. R. Rechenberg, G. Schneider, V. Villas-Boas, J. M. Moreau, L. Paccard, and J. P. Nozières, "Magnetic and structural characterization of $\mathrm{Nd}_{5} \mathrm{Fe}_{17}$ ", J. Appl. Phys. 70, 6125 (1991).

[103] S. Legvold, In E. P.Wohlfarth, editor, Ferro-Magnetic Materials, Vol. 1, pages 183-. North-Holland, Amsterdam, 1980.

[104] J. F. Löffler, H. B. Braun, and W. Wagner, "Magnetic Correlations in Nanostructured Ferromagnets", Phys. Rev. Lett. 85, 1990 (2000).

[105] J. F. Löffler, J. Schroers, and W. L. Johnson, "Time temperature transformation diagram and microstructures of bulk glass forming $\mathrm{Pd}_{40} \mathrm{Cu}_{30} \mathrm{Ni}_{10} \mathrm{P}_{20}$ ", Appl. Phys. Lett. 77, 681 (2000).

[106] J. F. Löffler, W. Wagner, and G. Kostorz, "Grain-size dependence of intergranular magnetic correlations in nanostructured metals", J. Appl. Crystallogr. 33, 451 (2000).

[107] Y. Li, S. C. Ng, Z. P. Lu, Y. P. Feng, and K. Lu, "Separation of glass transition and crystallization in metallic glasses by temperature-modulated differential scanning calorimetry", Philos. Mag. Lett. 78, 213 (1998). 
[108] I. M. Lifshitz and V. V. Slyozov, "The kinetics of precipitation from supersaturated solid solutions", J. Phys. Chem. Solids 19, 35 (1961).

[109] D. V. Louzguine and A. Inoue, "Evaluation of the thermal stability of a $\mathrm{Cu}_{60} \mathrm{Hf}_{25} \mathrm{Ti}_{15}$ metallic glass", Appl. Phys. Lett. 81, 2561 (2002).

[110] S. W. Lovesey, Theory of Neutron Scattering from Condensed Matter, Clarendon Press, Oxford, 1984.

[111] S. Mader and A. S. Nowik, "Metastable Co-Au alloys: Example of an amorphous ferromagnet", Appl. Phys. Lett. 7, 57 (1965).

[112] O. Malcai, D. A. Lidar, O. Biham, and D. Avnir, "Scaling range and cutoffs in empirical fractals", Phys. Rev. E 56, 2817 (1997).

[113] S. V. Maleyev, "Small-angle multiple neutron scattering in fractal media", Phys. Rev. B 52, 13163 (1995).

[114] B. B. Mandelbrot, The fractal geometry of nature, W.H. Freeman \& Company, New York, 1982.

[115] P. Mangin, B. Rodmacq, and A. Chamberod, "Fractal structure of $\mathrm{TiH}_{2}$ aggregates in a CuTi matrix", Phys. Rev. Lett. 55, 2899 (1985).

[116] J. A. Marqusee and J. Ross, "Kinetics of phase transitions: Theory of Ostwald ripening", J. Chem. Phys. 79, 373 (1983).

[117] D. Martien, Introduction to AC Susceptibility, Quantum Design, 2003.

[118] R. W. McCallum, I. R. Fisher, N. E. Anderson, P. C. Canfield, M. J. Kramer, and K. W. Dennis, "Reentrant behavior in the temperature dependence of metamagnetic transitions in single crystal $\mathrm{Nd}_{6} \mathrm{Fe}_{13-x} \mathrm{Al}_{1+x}$ ", IEEE T. Magn. 37, 2147 (2001).

[119] M. McElfresh, Fundamentals of Magnetism and magnetic measurements. Featuring Quantum Design's magnetic property measurement system, Quantum Design, 1994.

[120] P. Meakin, "Diffusion-controlled cluster formation in 2-6-dimensions", Phys. Rev. A 27, 1495 (1983).

[121] P. Meakin, "Diffusion-controlled cluster formation in two and three and four dimensions", Phys. Rev. A 27, 604 (1983).

[122] P. Meakin, "Formation of fractal clusters and networks by irreversible diffusionlimited aggregation", Phys. Rev. Lett. 71, 1119 (1983).

[123] V. P. Menushenkov, A. S. Lileev, M. A. Oreshkin, and S. A. Zhuravlev, "Metastable nanocrystalline $\mathrm{A}_{1}$ phase and coercivity in Fe-Nd alloys", J. Magn. Magn. Mater. 203, 149 (1999). 
[124] Ch. V. Mohan, H. Kronmüller, and M. Kelsch, "Magnetic phase transition in two dimensions: An experimental study on a system of amorphous ultrathin-film multilayers", Phys. Rev. B 57, 2701 (1998).

[125] R. M. Moon, T. Riste, and W. C. Köhler, "Polarization analysis of thermal-neutron scattering", Phys. Rev. 181, 920 (1969).

[126] K. Moorjani and J. M. D. Coey, Magnetic Glasses, Elsevier, Amsterdam, 1984.

[127] H. Ohno, S. Kohara, N. Umesaki, and K. Suzuya, "High-energy x-ray diffraction studies of non-crystalline materials", J. Non-Cryst. Solids 293-295, 125 (2001).

[128] R. J. Ortega-Hertogs, A. Inoue, and K. V. Rao, "Evolution from random-axis Ising to Stoner-Wohlfarth type of hysteresis loops of the cluster glass $\mathrm{Fe}_{3} \mathrm{Nd}$ phase in bulk glassy $\mathrm{Nd}_{60} \mathrm{Fe}_{30} \mathrm{Al}_{10}$ hard magnets", Scripta Mater. 44, 1333 (2001).

[129] W. Ostwald, "Über die vermeintliche Isomerie des roten und gelben Quecksilberoxyds und die Oberflächenspannung fester Körper", Z. Phys. Chem. 34, 495 (1900).

[130] D. E. Polk and B. C. Giessen, In J. J. Gilman and H. J. Leamy, editors, Metallic Glasses, Vol. 1, page 1. ASM, Metal Park, Ohio, 1978.

[131] W. Que, C. H. Kam, Y. Zhou, Y. L. Lam, and Y. C. Chan, "Yellow-to-violet upconversion in neodymium oxide nanocrystal/titania/ormosil composite solgel thin films derived at low temperature", Appl. Phys. Lett. 90, 4865 (2001).

[132] A. P. Radlinski, E. Z. Radlinska, M. Agamalian, G. D. Wignall, P. Lindner, and O. G. Randl, "Fractal geometry of rocks", Phys. Rev. Lett. 82, 3078 (1999).

[133] L. Ratke and P. W. Voorhees, Growth and Coarsening. Ripening in Material Processing, Springer-Verlag, Berlin, 2002.

[134] J. V. Rau, V. Rossi Albertini, N. S. Chilingarov, S. Colonna, and M. di Michiel, "Observation of a new Co-F compound detected by very-high-energy x-ray diffraction during thermal decomposition of $\mathrm{CoF}_{3}$ ", Chem. Lett. 7, 664 (2002).

[135] M. Sahimi, Heterogeneous material, Springer Verlag, Berlin, 2003.

[136] I. A. Santos and S. Gama, "Evidence for the stable existence of the $\mathrm{Fe}_{2} \mathrm{Nd}$ phase in the Fe-Nd system", J. Appl. Phys. 86, 2334 (1999).

[137] P. W. Schmidt, "Small-angle scattering studies of disordered and porous and fraetal systems", J. Appl. Crystallogr. 24, 414 (1991).

[138] S. Schneider, "Bulk metallic glasses", J. Phys.: Condens. Matter 13, 7723 (2001).

[139] S. Schneider, A. Bracchi, K. Samwer, M. Seibt, and P. Thiyagarajan, "Microstructure-controlled magnetic properties of the bulk glass-forming alloy $\mathrm{Nd}_{60} \mathrm{Fe}_{30} \mathrm{Al}_{10} "$, Appl. Phys. Lett. 80, 1749 (2002). 
[140] W. Scholz, H. Forster, D. Suess, T. Schrefl, and J. Fidler, "Micromagnetic simulation of domain wall pinning and domain wall motion", Com. Mater. Sci. 25, 540 (2002).

[141] V. F. Sears, "Neutron scattering lengths and cross sections", Neutron News 3, 26 (1992).

[142] E. Sharon, M. G. Moore, W. D. McCormick, and H. L. Swinney, "Coarsening of Fractal Viscous Fingering Patterns", Phys. Rev. Lett. 91, 205504 (2003).

[143] T. D. Shen and R. B. Schwarz, "Bulk ferromagnetic glasses prepared by flux melting and water quenching", Appl. Phys. Lett. 75, 49 (1999).

[144] C. G. Shull and M. K. Wilkinson, "Neutron scattering lengths and cross sections", Phys. Rev. 97, 304 (1955).

[145] L. Si, J. Ding, L. Wang, Y. Li, H. Tan, and B. Yao, "Hard magnetic properties and magnetocaloric effect in amorphous NdFeAl ribbons", J. Alloy Compd. 316, 260 (2001).

[146] S. K. Sinha, "Scattering from fractal structures", Phys. D 38, 310 (1989).

[147] F. Spizzo, E. Angeli, D. Bisero, A. da Re, F. Ronconi, P. Vavassori, I. Bergenti, A. Deriu, A. Hoell, and H. J. Lauter, "SANS measurements withpolarised neutrons on FeAg magnetic granular samples: compositional and magnetic morphology", J. Magn. Magn. Mater. 262, 124 (2003).

[148] H. E. Stanley, "Learning concepts of fractals and probability by "doing science"", Phys. D 38, 330 (1989).

[149] E. C. Stoner, Proc. R. Soc. London A 169, 339 (1939).

[150] J. Strohmenger, T. Volkmann, J. Gao, and D. M. Herlach, "Phase selection in undercooled Fe-Nd alloy melts", Mat. Sci. Eng. A 375-377, 561 (2004).

[151] Z. G. Sun, W. Löser, J. Eckert, K. H. Müller, and L. Schultz, "Magnetic properties of $\mathrm{Nd}_{60-x} \mathrm{Y}_{x} \mathrm{Fe}_{30} \mathrm{Al}_{10}(\mathrm{x}=0,10,30)$ melt-spun ribbons containing two amorphous magnetic phases", J. Appl. Phys. 91, 9267 (2002).

[152] Z. G. Sun, W. Löser, J. Eckert, K. H. Müller, and L. Schultz, "Phase separation in $\mathrm{Nd}_{60-x} \mathrm{Y}_{x} \mathrm{Fe}_{30} \mathrm{Al}_{10}$ melt-spun ribbons", Appl. Phys. Lett. 80, 772 (2002).

[153] Z. G. Sun, W. Löser, J. Eckert, K. H. Müller, and L. Schultz, "Effect of cooling rate on microstructure and magnetic properties of $\mathrm{Nd}_{60} \mathrm{Fe}_{30} \mathrm{Al}_{10}$ hard magnetic alloys", J. Magn. Magn. Mater. 261, 122 (2003).

[154] J. R. Taylor, An introduction to error analysis: The study of uncertainties in physical measurements, University Science Books, Sausalito, 1996. 
[155] J. Teixeira, "Small-angle scattering by fractal systems", J. Appl. Crystallogr. 21, 781 (1988).

[156] P. Thiyagarajan, J. E. Epperson, R. K. Crawford, J. M. Carpenter, T. E. Klippert, and D. G. Wozniak, "The Time-of-flight small-angle neutron diffractometer (SAD) at IPNS, Argonne National Laboratory", J. Appl. Crystallogr. 30, 280 (1997).

[157] M. F. Toney, K. A. Rubin, S. M. Choi, and C. J. Glinka, "Small-angle neutron scattering measurements of magnetic cluster sizes in magnetic recording disks", Appl. Phys. Lett. 82, 3050 (2003).

[158] D. Triyono, R. Sato Turtelli, R. Grössinger, H. Michor, K. R. Pirota, M. Knobel, H. Sassik, T. Mathias, S. Höfinger, and J. Fidler, "Temperature dependence of hysteresis loops and $\mathrm{AC}$-susceptibility of as-cast and annealed $\mathrm{Nd}_{60} \mathrm{Fe}_{30} \mathrm{Al}_{10}$ hard magnetic alloy", J. Magn. Magn. Mater. 242-245, 1321 (2002).

[159] C. C. Tsuei and P. Duwez, "Metastable amorphous ferromagnetic phases in palladium-base alloys", J. Appl. Phys. 37, 435 (1966).

[160] D. Turnbull, "Under what conditions can a glass be formed?", Contemp. Phys. 10, 473 (1969).

[161] R. Sato Turtelli, D. Triyono, R. Grössinger, H. Michor, J. H. Espina, J. P. Sinnecker, H. Sassik, J. Eckert, G. Kumar, Z. G. Sun, and G. J. Fan, "Coercivity mechanism in $\mathrm{Nd}_{60} \mathrm{Fe}_{30} \mathrm{Al}_{10}$ and $\mathrm{Nd}_{60} \mathrm{Fe}_{20} \mathrm{Fe}_{10} \mathrm{Al}_{10}$ alloys", Phys. Rev. B 66, 054441 (2002).

[162] R. Sato Turtelli, D. Triyono, G. Wiesinger, R. Grössinger, and H. Michor, "Local cooling rate dependence of magnetic properties in melt-spun $\mathrm{Nd}_{60} \mathrm{Fe}_{30} \mathrm{Al}_{10}$ alloy", IEEE T. Magn. 39, 2878 (2003).

[163] R. Sato Turtelli, D. Triyono, G. Wiesinger, M. Reissner, W. Steiner, J. P. Sinnecker, G. Badurek, and R. Grössinger, "Magnetic properties and Mössbauer effect study of $\mathrm{Nd}_{60} \mathrm{Fe}_{30} \mathrm{Al}_{10}$ ribbons", J. Alloy Compd. 369, 162 (2004).

[164] H. C. van de Hulst, Light scattering by small particles, Dover Publications, New York, 1981.

[165] T. Vicsek, Fractal Growth Phenomena, World Scientific, Singapore, 1992.

[166] C. Wagner, "Theorie der Alterung von Niederschlägen durch Umlösen (Ostwaldreifung)", Z. Elektrochemie 65, 581 (1961).

[167] W. Wagner, A. Wiedenmann, W. Petry, A. Geibel, and H. Gleiter, "Magnetic microstructure of nanostructured Fe, studied by small angle neutron scattering", Mat. Res. Soc. 6, 2305 (1991). 
[168] X. Z. Wang, Y. Li, J. Ding, L. Si, and H. Z. Kong, "Structure and magnetic characterization of amorphous and crystalline $\mathrm{Nd}-\mathrm{Fe}-\mathrm{Al}$ alloys", J. Alloy Compd. 290, 209 (1999).

[169] B. E. Warren, X-ray Diffraction, Addison-Wesley, London, 1969.

[170] B. C. Wei, W. H. Wang, M. X. Pan, B. S. Han, Z. R. Zhang, and W. R. Hu, "Domain structure of a $\mathrm{Nd}_{60} \mathrm{Al}_{10} \mathrm{Fe}_{20} \mathrm{Co}_{10}$ bulk metallic glass", Phys. Rev. B 64, 012406 (2001).

[171] J. Weissmüller, A. Michels, J. G. Barker, A. Wiedenmann, U. Erb, and R. D. Shull, "Analysis of the small-angle neutron scattering of nanocrystalline ferromagnets using a micromagnetics model", Phys. Rev. B 63, 214414 (2001).

[172] A. Wiedenmann, "Small-angle neutron scattering investigations of magnetic nanostructures using polarized neutrons", J. Appl. Crystallogr. 33, 428 (2000).

[173] A. Wiedenmann, "Small-angle neutron scattering investigations of magnetic nanostructures and interfaces using polarized neutrons", Phys. B 297, 226 (2001).

[174] A. Wiedenmann, A. Hoell, and M. Kammel, "Small-angle scattering investigations of cobalt-ferrofluids using polarised neutrons", J. Magn. Magn. Mater. 252, 83 (2002).

[175] C. G. Windsor, "An introduction to small-angle neutron scattering", J. Appl. Crystallogr. 21, 582 (1988).

[176] T. A. Witten and L. M. Sander, "Diffusion-limited aggregation and a kinetic critical phenomenon", Phys. Rev. Lett. 47, 1400 (1981).

[177] R. W. G. Wyckoff, Crystal Structures, John Wiley \& Sons, New York, 1964.

[178] F. Y. Yang, C. L. Chien, X. W. Li, G. Xiao, and A. Gupta, "Critical behavior of epitaxial half-metallic ferromagnetic $\mathrm{CrO}_{2}$ films", Phys. Rev. B 63, 092403 (2001).

[179] J. Zarzycki, Glasses and the vitreous state, Cambridge University Press, Cambridge, 1991.

[180] Z. G. Zhao, F. R. de Boer, and K. H. J. Buschow, "An introduction to small-angle neutron scattering", J. Alloy Compd. 239, 147 (1996).

[181] Z. G. Zhao, F. R. de Boer, V. H. M. Duijn, K. H. J. Buschow, and Y. C. Chuang, "Possible ferrimagnetic coupling in light-rare-earth transition-metal intermetallic compounds", J. Appl. Phys. 75, 7717 (1994). 


\section{Publications}

\section{Articles}

- A. Bracchi, K. Samwer, J. Almer, R. Aravinda Narayanan, P. Thiyagarajan, and S. Schneider "Separation of grain growth and glass transition during structural evolution of the bulk $\mathrm{Nd}_{60} \mathrm{Fe}_{30} \mathrm{Al}_{10}$ alloy", Phys. Rev. B 70, 172105 (2004).

- A. Bracchi, K. Samwer, T. Niermann, M. Seibt, and S. Schneider "Phase separation and magnetic properties of $\mathrm{Nd}_{60} \mathrm{Fe}_{30} \mathrm{Al}_{10}$ thin films", Appl. Phys. Lett. 85, 2565 (2004).

- A. Bracchi, K. Samwer, S. Schneider, P. Schaaf, and J.F. Löffler, "Phase separation and microstructure controlled magnetic properties of rapidly quenched $\mathrm{Nd}_{60} \mathrm{Fe}_{30} \mathrm{Al}_{10} "$, Mater. Sci. Eng. A 375-377, 104 (2004).

- A. Bracchi, S. Schneider, P. Thiyagarajan, and K. Samwer, "Cooling rate dependent microstructure and magnetic properties of the glass-forming alloy $\mathrm{Nd}_{60} \mathrm{Fe}_{30} \mathrm{Al}_{10}$ ", J. Magn. Magn. Mat. 272-276, 1423 (2004).

- A. Bracchi, K. Samwer, S. Schneider, and J.F. Löffler, "Random anisotropy and domain-wall pinning process in the magnetic properties of rapidly quenched $\mathrm{Nd}_{60} \mathrm{Fe}_{30} \mathrm{Al}_{10} "$, Appl. Phys. Lett. 82, 721 (2003).

- S. Schneider, A. Bracchi, K. Samwer, M. Seibt, and P. Thiyagarajan, "Microstructure-controlled magnetic properties of the bulk glass-forming alloy $\mathrm{Nd}_{60} \mathrm{Fe}_{30} \mathrm{Al}_{10}$ ", Appl. Phys. Lett. 80, 1749 (2002). 


\section{Conferences}

- Tagung der Deutscher Physikalischer Gesellschaft, Regensburg (Germany) 2004.

- Meeting of the Material Research Society (MRS Fall Meeting), Boston (USA) 2003.

- NSF CHEMBIO Workshop, Tallahassee (USA) 2003.

- International Conference on Magnetism (ICM03), Rome (Italy) 2003.

- Tagung der Deutscher Physikalischer Gesellschaft, Dresden (Germany) 2003.

- Rapid Quenched and Metastable Material (RQ11), Oxford (U.K.) 2002.

- Tagung der Deutscher Physikalischer Gesellschaft, Regensburg (Germany) 2002.

\section{Invited Talks}

- ETH, Zürich (Switzerland) 2004

- SFB 602, Göttingen (Germany) 2004

- Department of Materials Science, Oxford (U.K.) 2003

- SFB 602, Göttingen (Germany) 2003

- IFW, Dresden (Germany) 2003

- Dipartimento di Fisica, Bologna (Italy) 2001 


\section{Acknowledgments}

In the course of the past three years as $\mathrm{PhD}$ at the I. Physikalisches Institut of the University of Göttingen, many people have contributed to my work. Without their help and their constant encouragement, this thesis probably would not have seen the light of day and my thanks go to all of them. Particularly, I would like to mention:

PD Dr. Susanne Schneider for being my supervisor (Doktormutter) and to have created a friendly and stimulating research atmosphere which has been of invaluable importance for my work. I am deeply grateful to Susanne since she has constantly sustained and given me the scientific independence which I needed to complete this $\mathrm{PhD}$. I cannot imagine the three years I spent in Göttingen without her daily encouragements and suggestions. I am also in debt to Susanne for the opportunity to present my results in numerous national and international meetings, and for being a critical co-author of all my publications.

Prof. Dr. Konrad Samwer, who gave me the possibility to work at the I. Physikalisches Institut and supported my research in "good" as well as in "bad" times. I am indebted to Prof. Samwer for the opportunity to constantly face new challenges and valuable new experiences, which formed me not only as a "scientist" but also as a person. I would also like to thank Prof. Samwer for being my second referee.

Prof. Dr. Wolfgang Felsch, for the innumerable fruitful discussions and his suggestions.

PD Dr. Michael Seibt and Tore Niermann, who carried out the TEM characterization of the $\mathrm{Nd}_{60} \mathrm{Fe}_{30} \mathrm{Al}_{10}$ samples. The tendency of the rare-earth elements to oxidize has transformed their work in a serious scientific challenge. Nevertheless, they were able to produce outstanding TEM pictures and valuable results which have strongly contributed to this work.

PD Dr. Peter Schaaf, for the Mössbauer measurements and for being an active coauthor of one of my publications [18].

Carsten Mahn and Uta Bete, to have introduced me to the most important step of an experimental $\mathrm{PhD}$ work: the samples preparation. I am also indebted to Katrin Gehrke and Kerstin Thöne for their help with the XRD and DSC measurements and for their kind welcome during my first weeks in Göttingen. Their readiness to understand my poor 
German and to answer my questions has been an important help to overcome all those difficulties which "der Italiener" had during the last three years.

The Werkstatt (Hartmut Eichenberg and Axel Bruchmann) and Joachim Feist for their help and the friendly atmosphere of which I have taken advantage in the breaks of my work.

Elmar Gatzemeier, for having taken actively part (...with me) in the work of the "VSM Quenching Team". I must admit that in a couple of occasions we made a really good job!

My office colleges during the last three years: Dr. Vasily Moshniaga ("Tovarisch" Vasily), Markus Esseling and Jan Petersen (the "Globulos Brothers"), Maria Tschuschke ("ich drehe durch!") and Iris Rösner. They are without doubt the most valuable colleagues that a PhD could have in Göttingen. I am particularly indebted to Markus and Jan ("Harry" and "Janny") for many helpful discussions and for having been the proofreaders of this thesis. Their help kept embarrassing mistakes seeing the light of day!

Beyond Göttingen, the collaboration with Dr. Pappannan Thiyagarajan (IPNS and APS, Argonne) has been an important help in the interpretation of the small-angle neutron scattering and he has been a valuable co-author in almost my publications 15, 18, 19, 139]. I will never forget the week which I spent with him at the CHRNS Lab measuring polarized SANS "fighting" snow storms and several experimental difficulties.

I am indebted also to Dr. Jon Almer of the APS Argonne who introduced me to highenergy $\mathrm{x}$-ray scattering measurements and to their analysis. His competent suggestions have been an invaluable help for the analysis and interpretation of the WAXS results.

Prof. Dr. Carl Krill (University of Ulm) for his competent suggestions and valuable emails which have been an important help during the drafting up of this work and some publications.

Special thanks go to Prof. Dr. Jörg F. Löffler (ETH Zürich). His interested collaboration has helped me to produce the first important results of this work and the first two publications [17, 18. I am indebt to Prof. Löffler for having given me the possibility to present my results at the ETH and, on this occasion, to visit an unforgettable city.

It has been a pleasure to have many colleagues here in Göttingen who have given me the chance to improve my German and the capability to drink beer. I must admit that I have probably improved the first but seem still to be a novice in the latter (...at least compared to them!)... I am grateful to all of them for the friendly atmosphere which they contributed to create.

My sincere thanks go also to Marco Bertelli, Eugenio Forzani and Stefano Virdis of the "Göttingen Italian Team".

Alexandra Maiwald and Gerda Brocks who made my stay at the institute easier, much 
nicer and, for sure, "witziger"!

Financial support has been provided by the Deutsche Forschungsgemeinschaft through the project A5 (Strukturbildung und magnetische Eigenschaften von inhomogenen glasbildenden Systemen) of the Sonderforschungsbereich 602 (Complex structures in condensed matter from atomic to mesoscopic scales) since January 2002, and by the state of Niedersachsen via the GraduAte School OF Physics which granted the Georg Cristoph Lichtenberg fellowship I have been benefiting since October 2001.

I would also like to thank my family (my parents and my sister), my "German family" (Mr. and Mrs. Böhmer, Mark and Aysu) and Zdenko Grobenski for their help, patience, and their sincere trust.

This work would probably not have come to an end without the loving support and the constant understanding of my girlfriend Mia. I dedicate this thesis to her. 



\section{Curriculum Vitae}

\section{Alberto Bracchi}

\begin{tabular}{|c|c|}
\hline \multicolumn{2}{|r|}{ Personal Information } \\
\hline Birthday & March 4th, 1972 \\
\hline Birthplace & Montegiorgio (AP), Italy \\
\hline Citizenship & Italian \\
\hline \multicolumn{2}{|r|}{ Education and Experience } \\
\hline 1978-1983 & Scuola Elementare Statale, Montegiorgio. \\
\hline $1983-1986$ & Scuola Media "G. Cestoni", Montegiorgio. \\
\hline $1986-1991$ & Liceo Scientifico "E. Medi", Montegiorgio. \\
\hline 1991 & Secondary School Exam, Final Mark 60/60 with honors. \\
\hline 1992-1999 & Student of physics, University of Bologna. \\
\hline 1998 & $\begin{array}{l}\text { Technical collaboration with the preparation of the book } \\
\text { "Misure e Complementi di Fisica", U.Valdrè, CLUEB (1998) } \\
\text { Bologna. }\end{array}$ \\
\hline 1998-1999 & Civil Service, Policlinic "S. Orsola", University of Bologna. \\
\hline 1999 & $\begin{array}{l}\text { Laurea in physics. Thesis work: "Relationship between } \\
\text { structural properties and magnetoelastic behavior in metallic } \\
\text { glasses: the system FeNiCoZrNbCuB". Final Mark 110/110. }\end{array}$ \\
\hline 2000 & $\begin{array}{l}\text { Practical Training at the I. Physikalisches Institut, University } \\
\text { of Göttingen (Germany), "Synthesis and characterization of } \\
\text { metallic glasses Zr-based, Fe-based, Pd-based". } \\
\text { Participation in the "Second Course of Vacuum Physics and } \\
\text { Technology", Lecce, Italy. }\end{array}$ \\
\hline
\end{tabular}


2000-2001 Practical Training at Alcatel Submarine Networks DiVISION, Greenwich (London, UK), "Development of a simple model by Finite Difference Method for studying the power dissipation in electronic boards", Leonardo Project of the European Union and of the University of Bologna.

2001 Scholarship of the University of Bologna for a course on metallic glasses at the I. Physikalisches Institut, University of Göttingen (Germany): "Synthesis and characterization of metallic glasses Nd-Fe-based".

10.2001-10.2004 PhD at the Göttingen Graduate School of Physics under financial support of Land Niedersachsen (Germany) through a "Georg Christoph Lichtenberg" fellowship.

\section{Contact}

Alberto Bracchi

via Faleriense Est 26

I-63025 Montegiorgio (AP)

Italy

E.m@il: alberto.bracchi@phys.uni-goettingen.de

alberto.bracchi@iol.it 


\title{
Lebenslauf
}

\author{
Name: $\quad$ Alberto Bracchi \\ Geburtsdatum: $\quad$ 04.03.1972 \\ Geburtsort: $\quad$ Montegiorgio (AP), Italien \\ Familienstand: $\quad$ ledig \\ Staatsangehörigkeit: italienisch \\ 1978-1983 Grundschule Montegiorgio \\ 1983-1986 Scuola Media “G. Cestoni”, Montegiorgio \\ 1986-1991 Gymnasium "E. Medi”, Montegiorgio \\ $1991 \quad$ Abitur \\ 1992-1999 Studium an der Universität Bologna Physik \\ 1998-1999 Zivildienst am Klinikum "S. Orsola" der Universität Bologna \\ 1999 Diplomarbeit bei Prof. Dr. E. Bonetti, Thema: "Relationship \\ between structural properties and magnetoelastic behavior in \\ metallic glasses: the system FeNiCoZrNbCuB" \\ 02.2000-08.2000 Auslandsstipendium der Universität Bologna am I. Physika- \\ lischen Institut der Universität Göttingen \\ 08.2000-02.2001 Praktikum am Alcatel Submarine Networks Division, Green- \\ wich (London, UK) \\ 02.2001-10.2001 Auslandsstipendium der Universität Bologna am I. Physika- \\ lischen Institut der Universität Göttingen \\ 10.2001-10.2004 Promotionsstudium Physik an der GötTingen GRADUATE \\ SCHOOL OF PhYsics der Universität Göttingen. Anfertigen \\ der vorliegenden Dissertation am I. Physikalischen Institut \\ der Universität Göttingen bei PD. Dr. Susanne Schneider und \\ Prof. Dr. Konrad Samwer
}


